\title{
Modeling the origins of spatial and temporal variability in visual cortical representations
}

\author{
PhD Thesis \\ in partial fulfillment of the requirements \\ for the degree Dr. rer. nat. \\ in the Neuroscience Program \\ at the Georg August University Göttingen \\ Faculty of Biology
}

submitted by

Juan Daniel Flórez Weidinger

born in

Bogotá, Colombia

Göttingen, September 9, 2013 


\section{Examination committee}

Prof. Dr. Fred Wolf

(Thesis supervisor and 1st reviewer)

Department of Nonlinear Dynamics, Max Planck Institute for Dynamics and Self-Organization,

Bernstein Center for Computational Neuroscience, Göttingen

Prof. Dr. Dr. Detlev Schild

(2nd reviewer)

Institute of Neurophysiology and Cellular Biophysics,

Georg August University, Göttingen

Prof. Dr. Stefan Treue

Cognitive Neuroscience Laboratory, German Primate Center, Göttingen

Georg August University, Göttingen

Date of the oral examination: 24th October 2013 


\section{Statement of originality}

I hereby declare that this dissertation entitled "Modeling the origins of spatial and temporal variability in visual cortical representations" has been written independently and with no other sources and aids than those cited.

Juan Daniel Flórez Weidinger

Göttingen, 9th September 2013 
To Melanie, my fallen angel dressed in black 


\section{Contents}

1 Introduction 1

1.1 The visual pathway . . . . . . . . . . . . . . . . . . 2

1.2 Organized orientation preference layouts $\ldots \ldots \ldots \ldots$

1.3 Models of orientation map formation . . . . . . . . . . . . . . . . 5

1.3.1 Definition of the order parameter model $\ldots \ldots \ldots \ldots$

1.3.2 The linear and cubic functional . . . . . . . . . . . . . . . . 8

1.3.3 Success of the order parameter model . . . . . . . . . . . . . . . 10

1.4 Disordered orientation preference layouts . . . . . . . . . . . . . . . . . . . 11

1.5 Previous approaches to understand the interspersed layout . . . . . . . . . . 13

1.6 Overview of this work . . . . . . . . . . . . . . . . . . . . 17

2 Understanding the formation of interspersed layouts in visual cortical networks 20

2.1 Generalization of the model . . . . . . . . . . . . . . . . . . . . . . 21

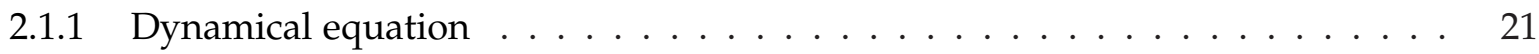

2.1 .2 Describing the interactions . . . . . . . . . . . . . 22

2.1.3 The model generates ordered and disordered layouts . . . . . . . . . . . 23

2.2 Analytical solution of the model in $1 \mathrm{D} \ldots \ldots \ldots \ldots$

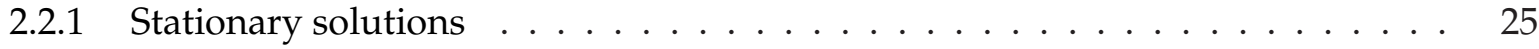

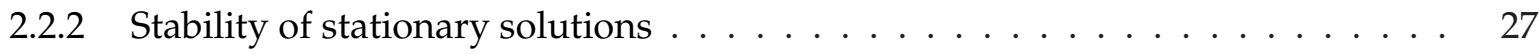

2.3 The transition from order to disorder . . . . . . . . . . . . . . . . . . . 31

2.3.1 Simplifying interaction parameters . . . . . . . . . . . . . 31

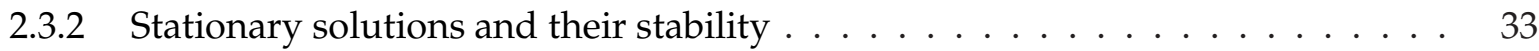

2.3 .3 Numerical confirmation of results . . . . . . . . . . . . . . . . . 37

2.4 Main conclusions from the order-parameter model . . . . . . . . . . . . . . . . 42

2.5 Strong intracortical inhibition in a LGN correlation based model . . . . . . . . . . . 43

2.5.1 Introduction to the model . . . . . . . . . . . . . . . . . . . . . 43 
2.5.2 The equations of the model . . . . . . . . . . . . . . 45

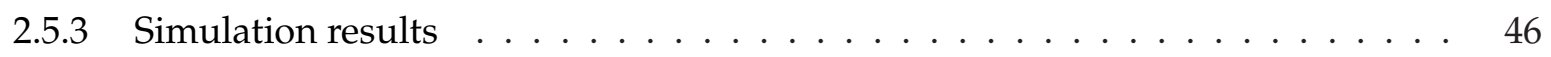

3 Model for binocular neurons $\quad 49$

3.1 Construction of the model . . . . . . . . . . . . . . . . . . . 50

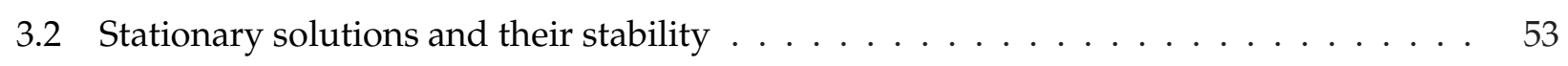

3.2 .1 Unselective state . . . . . . . . . . . . . . . . . . . . . . 54

3.2.2 Monocular orientation selective state . . . . . . . . . . . . . 54

3.2.3 Binocular orientation selective state . . . . . . . . . . . . . 55

3.3 Single parameter conditions for late orientation preference matching $\ldots \ldots$. . . . 58

3.4 Including spatial interaction $\ldots \ldots \ldots \ldots \ldots \ldots$

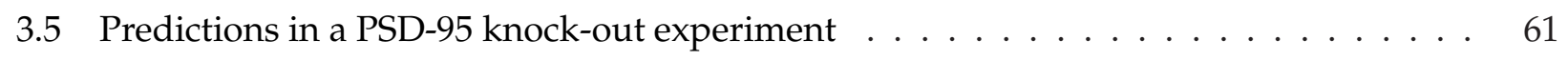

3.5 .1 Tuning of the model $\ldots \ldots \ldots \ldots \ldots$

3.5 .2 Numerical results . . . . . . . . . . . . . . . . . . . . 64

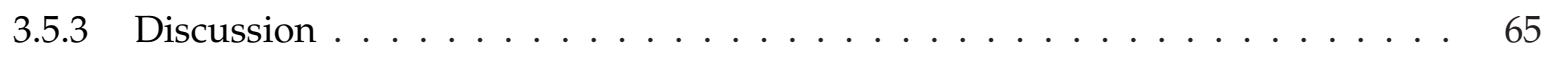

$4 \quad$ Functional implications of the interspersed layout $\quad 67$

4.1 Dynamical characterization . . . . . . . . . . . . . . . 68

4.1 .1 Glassy dynamics . . . . . . . . . . . . . . . . . . . 69

4.1 .2 Energy landscape . . . . . . . . . . . . . . . . . . 71

4.1.3 Lability of preferred orientations in the interspersed phase . . . . . . . . 73

4.2 spatial characterization . . . . . . . . . . . . . . . . 75

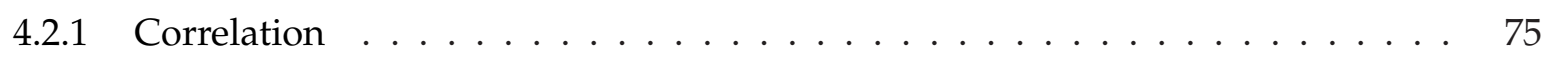

4.2 .2 Discrepancy . . . . . . . . . . . . . . . . 77

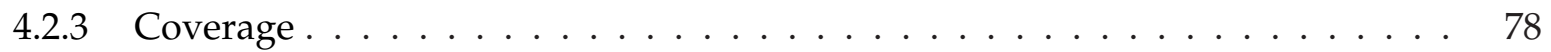

5 Understanding evolutionary transitions between the different layout types 83

5.1 Area size and functional layout . . . . . . . . . . . . . . 83

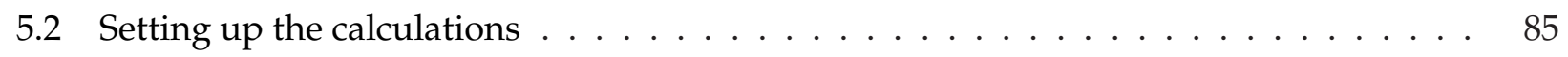

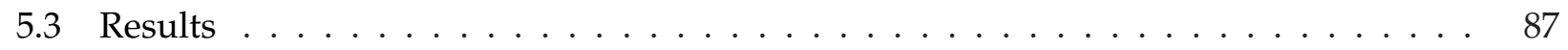

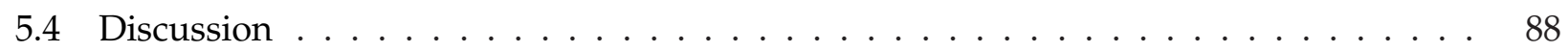

6 Estimation of tuning curves in the rat visual cortex 90

6.1 Experimental protocol and tuning curve estimation . . . . . . . . . . . . . . . . . . . . .

6.2 Comparison of the stimulation and estimation methods . . . . . . . . . . . . 93 


\section{CONTENTS}

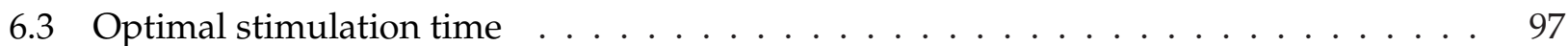

6.4 Non-stationarity of the preferred orientation $\ldots \ldots \ldots \ldots$

6.5 Discussion . . . . . . . . . . . . . . . . . . . . . . . 102

7 Summary and discussion $\quad 104$

Bibliography 112

$\begin{array}{lr}\text { Acknowledgements } & 128\end{array}$ 



\section{Chapter 1}

\section{Introduction}

The brain is an amazing organ. It consist of billions of neurons, most of which have thousands of connections from and to others. This complex entanglement of interacting units defines us as individuals, determining how we sense the world and interact with it. Our brain governs our inner world, modulating our emotions, our instincts, giving us dreams [1].

The field of neuroscience is very broad. Research can be focused from single molecules inside one cell to systems of millions of interacting neurons. All those different scales are not independent of each other, but have direct and reciprocal causal links. For example, mutations of a small molecule like the amyloid precursor protein can lead to a system disease like Alzheimer [2], or conversely a prolonged behavioral response to social stress can lead to the alteration of the number of serotonin receptors in the membrane of the neurons [3]. This makes the brain a very complex system, such that all types of approaches are necessary to understand it.

What is the role of a theoretical approach to neuroscience? What can be learned from the mathematical models? Theoretical models have different abstraction levels. For a given phenomenon, the model can be based on biophysical rules, illustrating the different processes in detail with many free parameters. The goal of theory then is to reduce the system to a small number of key parameters that play a fundamental role in the system and to understand their relation. On the other hand, a model can be very abstract, proposing an equivalent simplified system with similar properties with only a small number of parameters.

The behavior of the abstract model can nevertheless be linked to the original system and help to better understand the phenomenon. Independent of the level of abstraction, all models are caricatures of reality, constructed upon some assumptions that link the theory to the biology [4]. The aim of analyzing models of nature in general is to understand the fundamental principles of the underlying mechanisms in the observed phenomenon starting from those assumptions and to pose new questions to deepen its understanding [5].

Theory has brought many insights in understanding different aspects of neuroscience [6]. Known examples are the model for action potential initiation [7] or the formulation of activity dependent learning rules for the interaction between the neurons [8]. Both models were later deepened experimentally, elucidating the cellular and molecular basis of the phenomenon, for example in Neher et al. [9] and [10] respectively. Visual neuroscience has also benefited from the theoretical approach [11]. For example, the existence of pinwheels in the visual cortex was predicted in a model [12] and 
later confirmed experimentally $[13,14]$. The role of correlation between the eyes and inhibition in the cortex in determining the width of the ocular dominance columns was proposed theoretically $[15,16]$ and later tested in the lab $[17,18]$. Or as will be described further down in this thesis, the density of pinwheels in the visual cortex was predicted in a self-organization model [19] and later confirmed experimentally for different species [20].

This thesis deals with the phenomenon of pattern formation in the primary visual cortex of eutherian mammals. I will use the self-organization model developed by Wolf [21, 22] as basis and build on it to increase our understanding of the principles that lead to the formation of disordered layouts of orientation preference, which are found in some mammalian species and can not be explained by any existing dynamical model of neuronal pattern formation. Based on the results of the model I will make some predictions on the spatial statistics and tuning dynamics of the disordered layout that can be tested experimentally. I will also introduce an optimization model that can explain why some species have ordered layouts and others don't.

To put the phenomenon of disordered orientation layouts in context first a brief overview of the visual system will be presented. Then ordered orientation preference layouts will be introduced, followed by the description of the dynamical model proposed by Wolf [22] that can explain their formation and statistical invariance across species. Afterwards, the phenomenon of disordered layouts of orientation preference found in some species and the approaches taken so far to characterize and explain their formation will be presented.

\subsection{The visual pathway}

The visual pathway is roughly invariant in all mammas, where the difference lies mostly in the number and type of neurons involved. In this chapter the pathway in primates is explained, which is commonly used in the textbooks as a well characterized example [? ]. The visual pathway starts in the retina in the back of the eyes, where photons excite the opsins of cones and rods starting a reaction cascade that stops the influx of cations into the cell and leads to its hyperpolarization. The signal is transmitted and combined in the retina through non-spiking neurons like the bipolar, horizontal and amacrine cells. Retinal ganglion cells (RGC) sample selectively the changes in activity from a population of those cells and transmit the information in the form of action potentials outside of the retina through the optic nerve. In primates the three main types of RGCs are parasol, which sample from rods and responds to movement and depth, midget, which sample from cones and encodes red and green color and forms, and koniocellular, which encodes blue color. In reality the subtypes of RGC are more abundant, with more than 20 types described in macaques.

After a cross over in the optic chiasm of around half of the fibers coming from the nasal side of the retina, RGC connect roughly to the same number to neurons in the dorsal lateral geniculate nucleus (dLGN) of the thalamus. Other targets of the optic nerve are several subcortical regions like the superior colliculus or the pretectal nucleus which mediate reflexive eye movements and pupil dilation respectively. In the dLGN there is a clear anatomical separation of cell bodies in six layers, where fibers coming from ipsi and contra-lateral eye are in layers 2, 3, 5 and in layers 1, 4, 6 respectively. Fibers coming from parasol RGC go to layers 1 and 2, fibers from midget RGC go to layers 3, 4, 5,6 , and fibers from koniocellular RGC connect in between the layers. The receptive fields in the dLGN are similar to the ones of RGC: approximately circular symmetric with center-surround struc- 


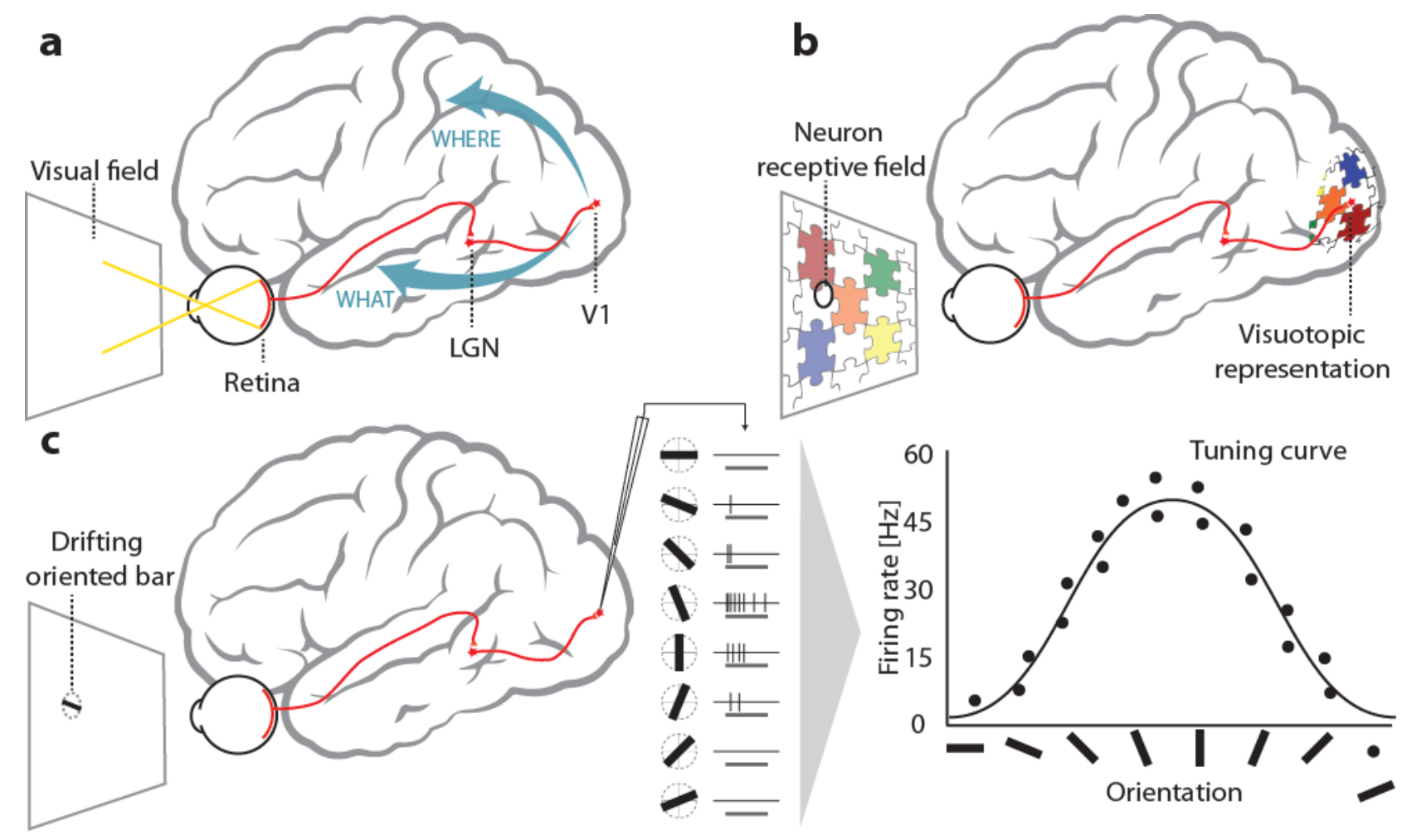

Figure 1.1.1: The visual pathway. a) Light enters the eye and is converted into electrical signals in the retina. The optic nerve have a relay in the dorsal lateral geniculate nucleus (LGN) and from there connect to neurons in the primary visual cortex (V1). After that point the pathway splits in two. The dorsal stream, known as the "Where Pathway", encodes the movement and position of the stimuli. The ventral stream, known as the "What Pathway", encodes the shape of the objects. b) In the primary visual cortex the receptive fields of neurons are organized topographically in a visuotopic map. Neighboring neurons encode neighboring locations in space. Notice that the representation of the visual field is inverted in the cortex. c) Neurons in the primary visual cortex are selective to the orientation of contours in their receptive field. Depending on the orientation of the bar, the example neuron modulates its firing rate. Brought together the firing rate describes a tuning curve, showing that the neuron has a preferred orientation of the stimuli in its receptive field.

ture, where ON cells fire the most when the center of the receptive field is activated by light and the surround not, and OFF cells fire the most when the opposite happens.

After the synaptic relay in the thalamus the pathway continues through the optic radiation to the posterior side of the neocortex in the Brodmann area 17, also called primary visual cortex, striate cortex or V1. In primates the number of neurons in V1 is thousandfold higher than the number of neurons in dLGN. The spatial organization of the optic fibers is maintained, generating a retinotopic (a.k.a. visuotopic or topographic) organization of receptive fields in the cortex, as shown in figure 1.1.1b). This means that neighboring neurons encode neighboring positions in visual space. The cortex in the left hemisphere responds to the right side of the visual field and the cortex in the right hemisphere to the left visual field. The binocular region is split in the most temporal part of both cortices. Both hemispheres are connected by fibers that run through the corpus callosum.

The primary visual cortex is often considered as the first stage of true visual processing. It is the first instance in the visual pathway where neurons respond to stimuli coming from both eyes [23]. How- 
a

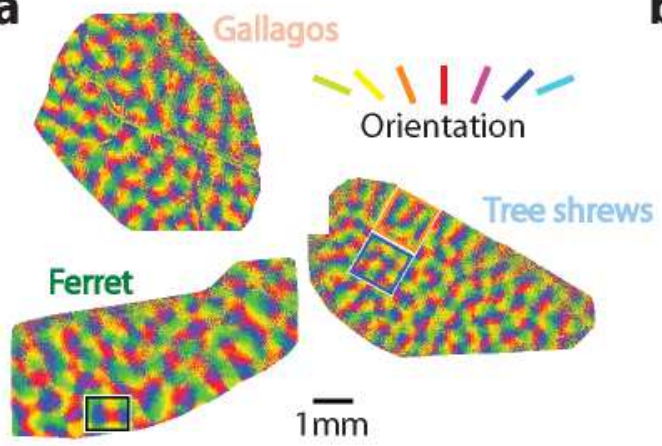

b

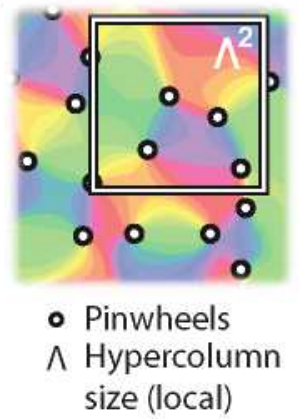

C

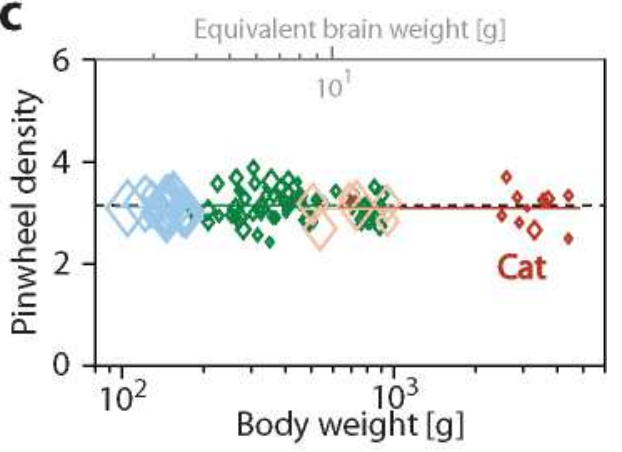

Figure 1.2.1: Orientation maps of different species have a common design. a) Orientation map of the gallago (primate), ferret (carnivore) and tree shrew (scadentia) obtained with intrinsic signal imaging. The preferred orientation of the neurons is color coded as shown. Modified from [20]. b) and c) The pinwheel density is conserved in species with maps. b) To calculate the pinwheel density the spatial coordinates are normalized by the local hypercolumn size. The pinwheel density is defined as the average number of pinwheels inside an hypercolumn squared area. c) Average pinwheel density for different animals. The color of the pointers correspond to the colors of the example maps in a). The pinwheel density approaches the natural constant $\pi$. b) and c) modified from [27].

ever, the strength of the response varies between the eyes, with some neurons firing preferentially to the left or the right eye. This property of the neurons is called ocular dominance. The shape of the receptive fields changes in V1 compared to RGC and neurons in dLGN. It becomes elongated with interchanging intervals of $\mathrm{ON}$ and OFF sub-regions. Therefore, neurons in V1 fire the most for a high contrast visual contour or bars of the same orientation as the elongated regions in the receptive field. This is illustrated in figure 1.1.1c). Depending of the orientation of the bar in the receptive field, the example neuron modulates its firing rate. Brought together the firing rate defines a tuning curve for orientation. This property of the neuron is called orientation preference. There is a distinction between simple and complex neurons, where in simple neurons the position of the bar inside the receptive field and not only its orientation modulates the firing rate and in complex cells the position is not of great influence [23]. Other functional properties of neurons in V1 that are not the main focus of this thesis are direction preference, spatial frequency tuning and color selectivity (see e.g. [24]).

The visual pathway continues in the extrastriate cortex in two streams. The first stream is known as the "Where Pathway" and continues in the dorsal part of the brain involving areas like V5. This stream has a direct connection to motor behavior and is associated with the location and motion of the stimulus (see e.g. area MT [25]). The second steam is known as the "What Pathway" and continues in the ventral part of the brain to the temporal cortex involving areas like V4. This stream is associated with encoding the shape of the stimulus and plays a role in the recognition of objects (see e.g. area IT [26]). As the visual pathway continues downstream through those streams the receptive fields become larger and more complex.

\subsection{Organized orientation preference layouts}

In the previous section the orientation selectivity of the neurons in the primary visual cortex was introduced. How does the orientation preference of neighboring neurons relate to each other? In 
many species a columnar architecture is found, where the neurons in the same radial axis through all the layers of the cortex share the same orientation preference. Parallel to the cortical surface the orientation preference of the columns varies continuously and smoothly in space [23]. The transition of preferred orientations exhibits singularities called pinwheels, where the orientation is radially arranged [12-14]. This kind of organized layout is referred to as orientation map and is found in cats [14, 28], ferrets [29-31], sheep [32], squirrel monkey [33, 34], macaques [23, 35], owl monkey [36], gallago [37], humans [38] and tree shrews [39]. Figure 1.2.1a) shows orientation maps for three different species obtained with intrinsic signal imaging [40]. The preferred orientations of the populations of neurons are labeled with colors. The orientation maps show a quasi-periodic arrangement with a typical spacing between columns preferring the same orientation in the millimeter range. This spacing can be defined as the linear size of an hypercolumn, which is defined as one set of columns showing all orientation preferences.

The species having ordered maps belong to different animal orders widely separated in evolution (primates, carnivores and scadentia), live in different ecological niches and span a large range of cortex sizes and shapes. How similar are their orientation maps? To remove the variation in spatial scales between species and inside each map, the unit of distance in the cortex can be renormalized by using the average local hypercolumn size [20]. Once this is done, the different orientation maps become statistically very similar. For example, as shown in figure 1.2.1b) and c), the mean number of pinwheels inside an hypercolumn area, known as the pinwheel density, clusters around an invariant density of $\sim 3.1$ for all species. As shown in [20], this invariant density is not a property of any arbitrary quasi-periodic arrangement of orientations, as randomized maps have a significantly higher density and many models predict distinct densities [41, 42]. Other pinwheel statistics like the count fluctuations of the density for increasing area size or the distance to nearest neighbors are also invariant, as shown in figure 1.3.1 d) to f), and are also different in randomized maps. This result suggest that the map in all those species follows a specific single common design.

\subsection{Models of orientation map formation}

The emergence of functional organized patterns in the visual cortex is an intriguing phenomenon in neuroscience. How do millions of neurons determine their preferred orientation and organize in the map? Soon after the discovery of orientation preference Hubel and Wiesel proposed that the connections between the neurons in the dLGN and the cortex necessary to shape the receptive fields are determined innately [43]. Indeed the specific sampling of dLGN afferents plays a fundamental role in the orientation selectivity [44] and a rough orientation map develops before visual experience [45]. On the other hand, this idea of a genetically predetermined network is not easy to reconcile with the astronomical number of synapses that have to be specified and the high degree of plasticity that the neurons exhibit in response to manipulations like binocular and monocular deprivation [23]. In fact, closing both eyes but raising ferrets under natural illumination completely abolishes the formation of orientation preference maps [29]. Another counterargument is the fact that the statistics of the layout are so similar in evolutionary distant related species [20], meaning that the genetic instructions had to be conserved in detail through millions of years of evolution. This leads to the idea that the organization might be obtained dynamically by activity dependent self-organization mechanisms $[16,22,46-48]$. 
What are the principles of pattern formation in the cortex? What is required for an ordered map to develop? Modeling the emergence of columnar patterns has been a challenging task in theoretical neuroscience since their discovery. A large number of models have been proposed with different levels of abstraction (see $[11,49]$ for reviews of some models). In a detailed parameter rich approach, networks of a large number of neurons with feed-forward and lateral interactions with synaptic weights following activity-dependent learning rules have been simulated [16, 47, 50-54]. In an intermediate level of abstraction a simple optimization principle was proposed and the necessary dynamics that lead to its minimization were derived [48, 55-61]. In some cases there was no direct energy functional to optimize $[62,63]$, or the dynamics are substituted with annealing algorithms [64, 65]. The most abstract approaches taken was to use the general principles of pattern forming media and combine them with qualitative features of map formation to propose models for the development of feature maps [21, 22, 41, 42, 66-72]. Intriguingly, it is this last, most abstract line of investigation that has led to the quantitatively most accurate account.

In the next section the model developed by Wolf [22]is introduced. So far it is the only dynamical model that can reproduce to an accuracy of a few percent the pinwheel statistics observed in the experiments (see figure 1.2.1c)). The model that I will introduce to understand the formation of interspersed patterns is a generalization of this model.

\subsubsection{Definition of the order parameter model}

There are many features in the visual pathway that might play a role in the formation of orientation selectivity and its spatial organization in the cortex. For example, neurons in the cortex might be optimized to match the statistics of the visual world; the specific layout of retinal ganglion cells in the retina might bias the formation of orientation maps; correlations between neurons in the dLGN can trigger the self-organization of oriented receptive fields in cortical neurons; etc. Also inside the primary visual cortex there are many factors that can influence the details of the layout and the selectivities of the neurons, like the anatomical boundaries of the visual area or the presence of electrical synapses between neurons early in development. To make a model that includes all possible factors that determine the organization in the cortex is not feasible; and it is not sure how much one can learn from it due to the large number of unknown parameters it would contain. Instead, the approach developed by Wolf [21, 22] and presented here starts from a different point of view: If one knows the tuning of all neurons in the primary visual cortex at a given point in time $t$, can this information be used to infer how the distribution of orientations will look like at a point $t+\Delta t$ later? If the answer is yes, the nature of activity dependent refinement that acts on a given state depends strongly on this state. Describing the tuning properties of a neuron $i$ at position $\mathbf{x}_{\mathrm{i}}$ by a complex number $z_{i}=A_{i} e^{i 2 \theta_{i}}$, where $\theta_{i}$ encodes the preferred orientation and $A_{i}$ the tuning selectivity [22,66], if one assumes that the answer to the previous question is affirmative, then an equation of the form

$$
\tau_{z} \frac{\partial z}{\partial t}=\hat{F}[z(\cdot)]
$$

exists. This type of order parameter model for visual cortex organization doesn't try to explain through which mechanism the neurons become orientation selective, since selectivity is assumed as a basic property of the neurons. Instead it focuses on intra-cortical interactions and describes how the tuning of one neuron is modified by the neurons with which it interacts. It is assumed that all 
the other sub-cortical mechanisms that do play a role in the formation of the spatial layout of orientations are maintained, e.g. there is no over-representation of a specific angle in visual space [73]. Furthermore, to find an equation for $\hat{F}[z(\cdot)]$ that captures all the biology of intra-cortical mechanisms that shape the layout of orientation preferences is a very difficult task. For this a statistical physics concept called universality suggest that this might not be necessary. It states that the properties of many dynamical systems that fall into a wide class of models are independent of microscopic details. Therefore, many dynamical properties of such universality classes of systems can be learned by analyzing one representative of the class. Particularly suitable from a technical point of view is the simplest example. The approach taken by Wolf was to search the properties that $\hat{F}[z(\cdot)]$ must satisfy to belong to the same class of dynamical models as the mechanisms shaping the organization in the cortex, and use a representative equation for $\hat{F}[z(\cdot)]$ that can be treated analytically to learn about the properties and constrains of the biological mechanisms.

The properties $\hat{F}[z(\cdot)]$ must satisfy are symmetry requirements that are assumed to exist in the dynamics of the visual cortex where all neurons are treated as equal units independent of their position or orientation. First it imposes $\hat{F}$ to be symmetric under translations $\hat{T}_{\mathbf{y}}$

$$
\hat{F}\left[\hat{T}_{\mathbf{y}} z(\mathbf{x})\right]=\hat{F}[z(\mathbf{x}+\mathbf{y})]=\hat{T}_{\mathbf{y}} \hat{F}[z(\mathbf{x})]
$$

and rotations $\hat{R}_{\beta}$

$$
\hat{F}\left[\hat{R}_{\beta} z(\mathbf{x})\right]=\hat{F}\left[e^{2 i \beta} z\left(\left(\begin{array}{cc}
\cos (\beta) & -\sin (\beta) \\
\sin (\beta) & \cos (\beta)
\end{array}\right) \mathbf{x}\right)\right]=\hat{R}_{\beta} \hat{F}[z(\mathbf{x})]
$$

This means that the spatial component of the interaction between the neurons can only be distance dependent. Secondly, $\hat{F}$ being independent of the orientation of the neuron imposes a phase shift symmetry

$$
\hat{F}\left[e^{i \varphi} z(\mathbf{x})\right]=e^{i \varphi} \hat{F}[z(\mathbf{x})]
$$

This means that only terms of odd order in $z$ can be included, such as $\hat{F}[z]=\hat{L}[z]+\hat{N}_{3}[z]+\hat{N}_{5}[z]+$ h.o.t, where $\hat{N}_{i}[z]$ is the i'th order term. Finally, to maintain the values of $z$ complex and have all orientations represented in the solution, $\hat{F}$ can be chosen symmetric under permutations

$$
\hat{N}_{3}\left[z_{1}, z_{2}, z_{3}\right]=\hat{N}_{3}\left[z_{2}, z_{3}, z_{1}\right]
$$

Near the onset of pattern formation it is understood that it is sufficient to study the dynamics truncated at the third order

$$
\hat{F}[z]=\hat{L}[z]+\hat{N}_{3}[z]
$$

Both linear $\hat{L}[z]$ and nonlinear $\hat{N}[z]$ part must each satisfy all the symmetry constrains. The choice of the linear term $\hat{L}$ determines the possible patterns that are stationary solutions to the system and the nonlinear term $\hat{N}_{3}$ determines their stability. Following Wolf, in the next section first an example equation will be presented which can generate quasi-periodic patterns of orientation selectivities as found in the visual cortex of carnivores and primates. Then the requirements on the nonlinear term to make those patterns that mimic biological observations stable will be analyzed. 


\subsubsection{The linear and cubic functional}

The symmetries of the system (equations 1.3.1 to 1.3.4) span a subspace of spatially ordered solutions in the form of finite Fourier components called planforms

$$
z=\sum_{j=1}^{n} A_{j} e^{i \mathbf{k}_{j} \mathbf{x}}
$$

where $\mathbf{k}_{j}$ is the wavenumber of the planform and determines its periodicity. The linear term $\hat{L}[z]$ is chosen such that the periodicity of those components is fixed at a determined wavenumber $\left|\mathbf{k}_{j}\right|=k_{c}$

$$
\hat{L}_{S H}[z]=r z-\left(k_{c}^{2}+\nabla^{2}\right)^{2} z
$$

This operator is known as the Swift-Hohenberg operator and is commonly used in pattern formation physics to describe a system exhibiting a supercritical symmetry break from the homogeneous solution where a finite pattern periodicity $k_{c}$ emerges and other modes are suppressed [74, 75]. This imposes a critical circle in Fourier space of size $k_{c}$ on which the wave-vectors of the planforms must lie. Because of the permutation symmetry in equation 1.3.4 two anti-parallel modes are not simultaneously possible, avoiding solutions that become real [21]. For this reason, this type of solutions are called essentially complex planforms (ECP). A large set of such solution takes the form

$$
z=\sum_{j=1}^{n} A_{j} e^{i l_{j} \mathbf{k}_{j} \mathbf{x}}
$$

where the $\mathrm{n}$ wave vectors are distributed equidistantly on the upper half of the critical circle $\mathbf{k}_{j}=$ $k_{c}\left(\cos \left(\frac{j \pi}{n}\right), \sin \left(\frac{j \pi}{n}\right)\right)$ and $l_{j}= \pm 1$ determines if the mode is flipped to the lower half. Figure 1.3.1a) shows how the resulting pattern looks like with an increasing number of modes. With $n=1$ the layout is a simple plane wave, and for increasing $n$ the pattern becomes quasi-periodic and has a higher similarity to the measured patterns in primates and carnivores. This resemblance is not just qualitative, but also quantitative. In figure b) the pinwheel density (average number of pinwheels in an hypercolumn square space) is plotted against the number of active modes, i.e the maps in figure a). As the number of active modes increases, the density approaches the value of the natural constant $\pi$, which is consistent with the quantitative density measured in galagos, ferrets, three shrews and cats up to $2 \%[20,27]$.

Next, the conditions on the nonlinear term $\hat{N}_{3}[z]$ are found to make the high numbers of amplitudes $A_{j}$ of the modes stable. To check for the stability, amplitude equations can be used which describe how the different modes interact with each other

$$
A_{i}=r A_{i}-\sum_{j=1}^{n} g_{i j}\left|A_{j}\right|^{2} A_{i}-\sum_{j=1}^{n} f_{i j} A_{j} A_{j^{-}} A_{i^{-}}
$$

were the coefficients $g_{i j}=\left(1-\frac{1}{2} \delta_{i j}\right) g\left(\left|\alpha_{i}-\alpha_{j}\right|\right)$ and $f_{i j}=\left(1-\delta_{i j}-\delta_{i^{-} j}\right) f\left(\left|\alpha_{i}-\alpha_{j}\right|\right)$ depend only on the angle difference between the modes and the index $j^{-}$denotes the mode that is anti-parallel to $j$. With ECP as in equation 1.3.6 the last term drops out since anti-parallel modes are not present. The corresponding stationary amplitudes for the planforms is 
a

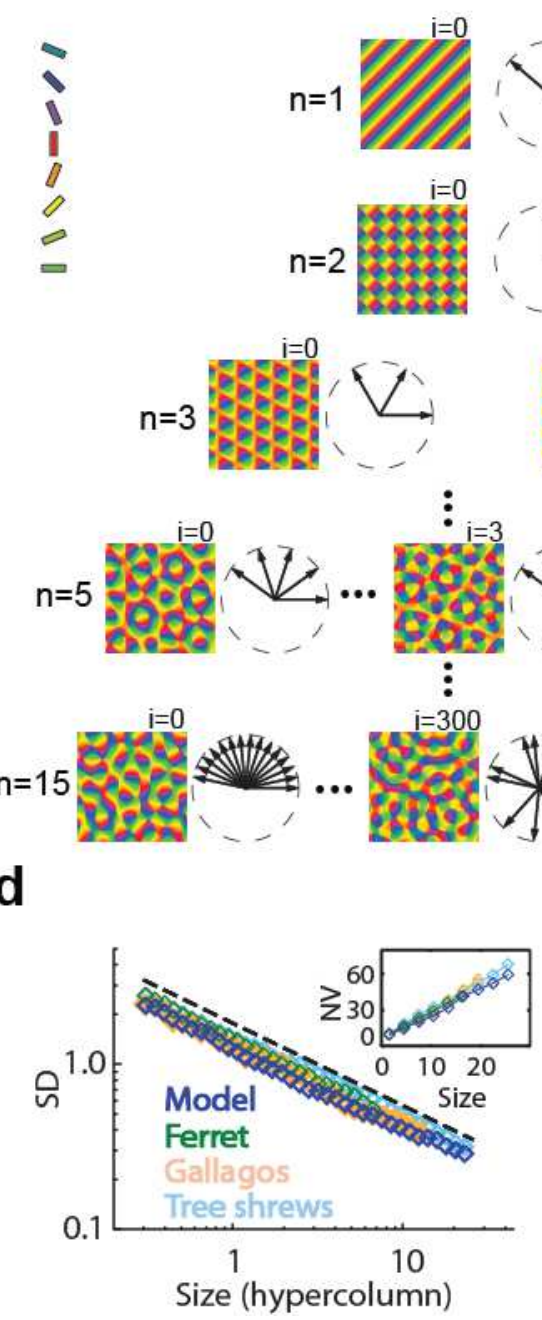

b

C
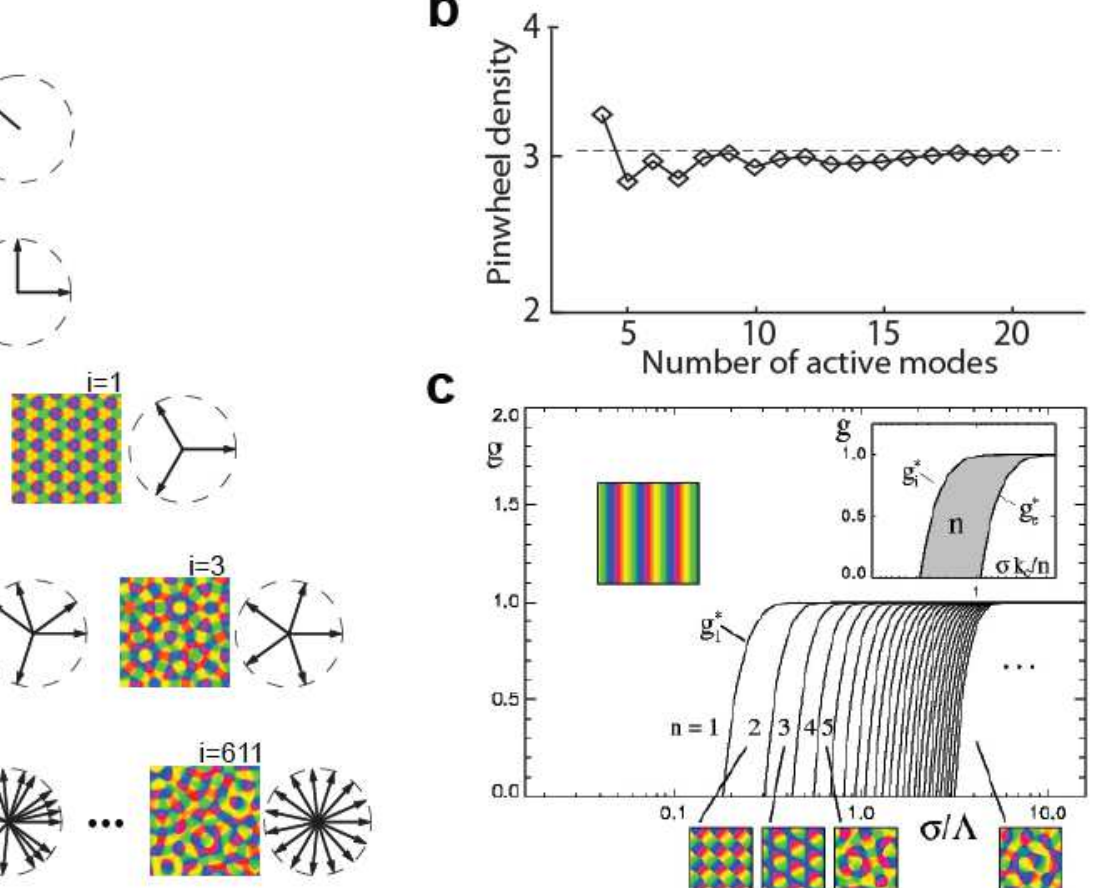

e

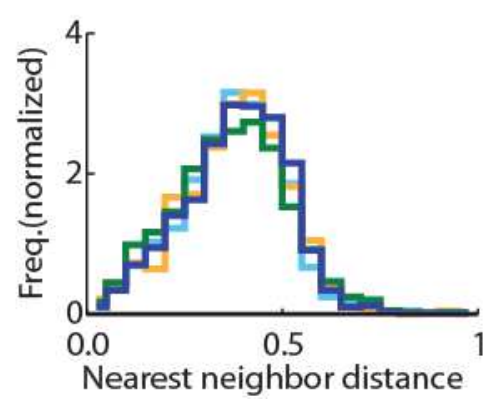

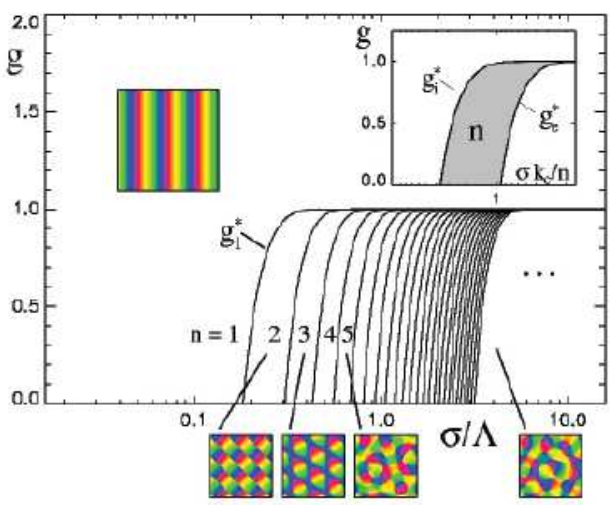

$\mathbf{f}$

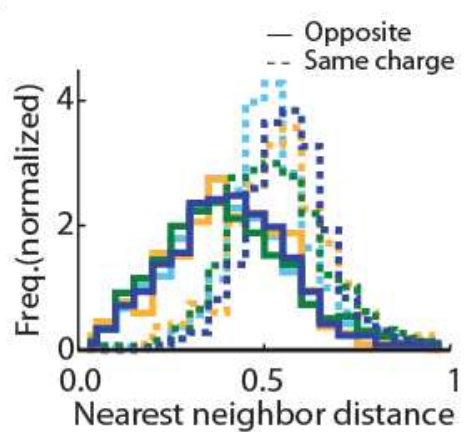

Figure 1.3.1: Summary of the long-range interaction model. a) Appearance of the pattern with an increasing number of complex planforms (ECP) on the critical circle. b) Average pinwheel density for an increasing number of active modes. The density approaches $\pi$, similar to the value found in experiments. c) Phase diagram of the stability of an n-ECP solution depending on the regulation of the influence of the local and nonlocal nonlinearities $g$ and the range of the orientation selective interactions $\sigma$. For long-range selective interactions pinwheel-rich maps are the solution of the model. d) to f) The model also replicates other common quantities found in species with maps: d) Standard deviation of pinwheel densities measured in areas of increasing size, e) frequency of distances between pinwheel nearest neighbors independent of their topological charge, f) frequency of distances between nearest neighbors for pinwheels of same and opposite charge separately. In e) and $\mathrm{f}$ ) the distance is normalized by the local hypercolumn size. 


$$
\left|A_{i}\right|^{2}=r \sum_{j=1}^{n}\left(\mathbf{g}^{-1}\right)_{i j}
$$

The equation of $g(\alpha)$ can be obtained for a general nonlinear functional $\hat{N}_{3}[z, z, \bar{z}]$ using multiscale expansion

$$
g(\alpha)=-e^{-i \mathbf{k}_{0} \mathbf{x}}\left[N_{3}\left(e^{i \mathbf{k}_{0} \mathbf{x}}, e^{i \mathbf{h}(\alpha) \mathbf{x}}, e^{-i \mathbf{h}(\alpha) \mathbf{x}}\right)+N_{3}\left(e^{i \mathbf{h}(\alpha) \mathbf{x}}, e^{i \mathbf{k}_{0} \mathbf{x}}, e^{-i \mathbf{h}(\alpha) \mathbf{x}}\right)\right]
$$

where $\mathbf{k}_{0}=k_{c}(1,0)$ and $\mathbf{h}(\alpha)=k_{c}(\cos (\alpha), \sin (\alpha))$ [21]. As a consequence of symmetry and the truncation at third order all solutions with the same number of modes $n$ have the same energy and stability properties, making it possible to use an example arrangement for $\mathbf{h}(\alpha)$ to determine the properties of the whole family. The stability of a ECP solution with $n$ modes can be calculated by inserting this in the equation above with a proposed nonlinear functional $\hat{N}_{3}[z]$. The functional chosen by Wolf is a combination of local and nonlocal terms

$$
\hat{N}_{3}[z]=(1-g)|z(\mathbf{x})|^{2} z(\mathbf{x})-(2-g) \int d^{2} y K_{\sigma}(\mathbf{x}-\mathbf{y})\left(|z(\mathbf{y})|^{2} z(\mathbf{x})+\frac{1}{2} \bar{z}(\mathbf{x}) z^{2}(\mathbf{y})\right)
$$

where for the shape of the nonlocal interactions a Gaussian kernel is used for simplicity

$$
K_{\sigma}(\mathbf{x}-\mathbf{y})=\frac{1}{2 \pi \sigma^{2}} e^{-\frac{(\mathbf{x}-\mathbf{y})^{2}}{2 \sigma^{2}}}
$$

This function is obtained by including orientation selective interactions between the neurons and expanding for low selectivity. This is similar to what will be used further down in this thesis. Only the terms that have an impact on the stability of the modes are maintained (see supplementary online material of [20]). The nonlinear part has to be negative to saturate the growth from the linear term, where $0 \leq g \leq 2$ regulates the influence of the local and nonlocal interaction. Inserting this $\hat{N}_{3}[z]$ in equation 1.3.7 leads to

$$
g(\alpha)=g+(2-g) 2 \cosh \left(k_{c}^{2} \sigma^{2} \cos \alpha\right) e^{-\sigma^{2} k_{c}^{2}}
$$

It depends on both $g$ and the range of the nonlocal interactions $\sigma$. Figure 1.3.1c) shows the n-ECP solutions that have minimal energy $U=-\frac{1}{2} \sum_{i, j}^{n}\left(\mathbf{g}^{-1}\right)_{i j}$ for a combination of $g$ and $\sigma$, where the last is normalized by the size of an hypercolumn $\Lambda$ that depends on the system size and $k_{c}$. Long-range orientation selective interactions have to be present to have a higher number of spatially complex planforms as an optimal solution. This kinds of interactions are biologically plausible, since axons connecting columns with the same orientation preference many hypercolumns away from each other have been reported $[39,76-79]$. Choosing the parameters $g$ and $\sigma$ in this regime makes quasi-periodic maps the attractors of the system.

\subsubsection{Success of the order parameter model}

The approach proposed by Wolf is the first model for orientation preference map development that shows analytically the existence and stability of quasi-periodic patterns that quantitatively resemble the maps measured in the experiments. Figure 1.3.1d) to f) shows that not only the observed pinwheel 
density is reproduced, but that other quantitative values that are conserved between the species with orientation maps also emerge from the results of the model [20]. In d) the conserved value is the standard deviation of pinwheel densities measured in areas of increasing size in units of $\Lambda^{2}$, in e) the frequency of distances between pinwheel nearest neighbors independent of their topological charge, and in f) the frequency of distances for pinwheels of same and opposite charge.

\subsection{Disordered orientation preference layouts}

That the orientation preferences are organized in space in some species comes as no surprise due to the high level of order in the visual pathway. Citing Hubel and Wiesel [23] :

"It is indeed hard to contemplate the nightmare of interconnections that would have to exist if the cells were distributed at random with respect to orientation."

All the seminal studies on the visual cortex were performed in species with high visual acuity and a pronounced vision driven behavior, like cats and monkey. Initially the visual system of rodents was not deeply investigated because their visual acuity is very low, 1.2 cycles per degree (cpd) in rat compared to $6 \mathrm{cpd}$ in cats and $46.0 \mathrm{cpd}$ in macaques [80]. Recently, advances in genetic tools for rodents have converted them in ideal model organisms to investigate the function of V1, as specific cells and neural circuits can be targeted, monitored and manipulated leaving the rest of the circuit unperturbed [81]. Rodents can perform behavioral task that relies on vision with high accuracy [82], such that the function of different network elements on the system level can be tested. Progressively more attention has been given to the rodent visual cortex, and both similitudes and differences with the standard model animals in visual science have been found.

Rodents lack a fovea in the retina, but the density of photoreceptor is higher in average than in macaques [83]. This means that they rely on head movements to track visual stimuli [84], where independent eye movements maintain the overhead binocular field fixed [85]. They exhibit 22 anatomically different subtypes of RGC [86], where almost half of them are motion selective [87]. A subtype of RGC selective to approaching stimuli has also been found [88]. Since the position of the eyes in rodents is lateral in the head, the binocular field of vision is much smaller than in primates and lies mostly in the upper part of the visual field. Accordingly, only about $10 \%$ of the optic fibers do not cross in the optic chiasm [89]. Downstream in the visual pathway, similar to primates, RGC in rodents connect to a high variety of subcortical targets, like the inter-geniculate leaflet, the suprachiasmatic nucleus, and the nuclei of the accessory optic tract [90]. In the dLGN, the receptive field of neurons is mostly $\mathrm{ON}$ and OFF center surround, with a significant fraction of neurons tuned for the orientation and direction of the stimulus [91-94]. This kind of oriented receptive fields are not an exclusive feature of rodents, since direction tuned neurons are also found in the dLGN of cat [95], rabbit [96] and marmoset [97].

As shown in figure 1.4.1a), in the primary visual cortex of rodents neurons have a visuotopic organization in the macroscopic scale [98]. At neuronal level the scatter in the receptive field position is large, with substantial receptive field sub-regions overlapping between neurons [101]. Although the binocular field in visual space is small, the binocular region in the cortex covers almost one third of the total V1 area [102]. The receptive fields of neurons in the cortex is remarkably similar in rodents compared to other mammals. As figure 1.4.1b) shows, once the degree of visual acuity is normalized, 
a

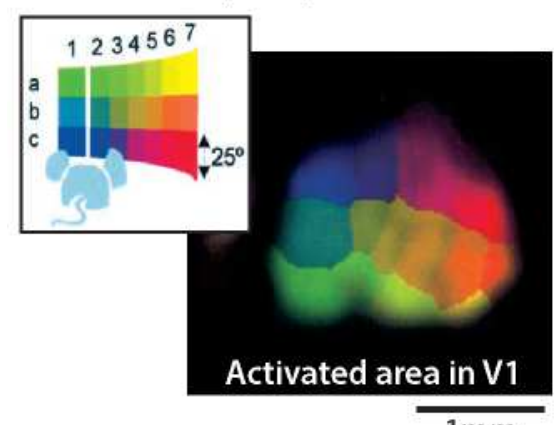

b

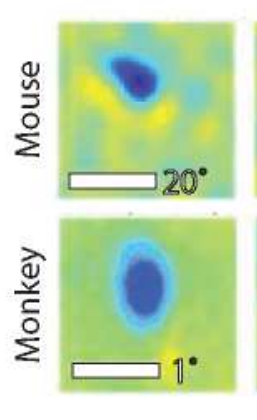

Orientation tuning

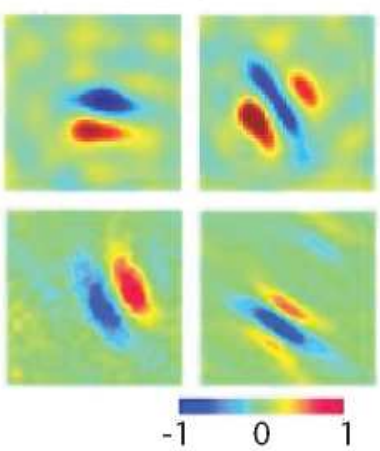

C Interspersed layout

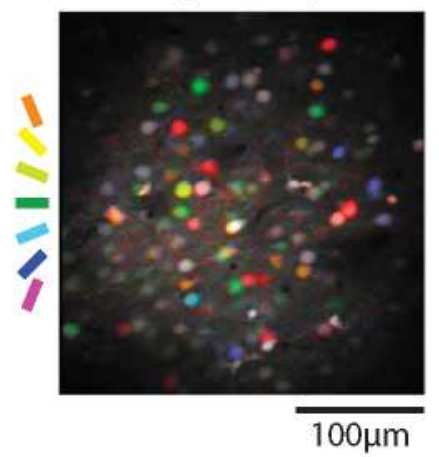

Figure 1.4.1: Neurons in the rodent visual cortex can represent orientated contours in the visual field, but preferred orientations are disordered in space. a) Visuotopic organization of receptive fields in mouse V1 obtained with intrinsic signal imaging. The color code in the cortex represents the position in the visual field as shown above. Modified from [98]. b) Example receptive fields for neurons in the mouse (top row) and monkey (bottom row). Notice that the scale bar in mouse is $20^{\circ}$ and in the monkey is $1^{\circ}$. Although the visual acuity is almost two orders of magnitude smaller in rodents, the shape of the receptive field is remarkably similar. Modified from [99]. c) Spatial layout of orientation preferences in a rat obtained with 2-photon calcium imaging. The preferred orientation is color coded. Although the neurons are tuned for orientation, an ordered orientation map is not present. Modified from [100].

receptive fields share many similar properties [81]. A very detailed characterization of neurons in mouse V1 was done in [103] and comparative review to other mammalian species is given in [80]. In mice $75 \%$ of neurons are tuned for orientation, just slightly lower than in primates. The spatial frequency is 0.04 cycles per degree (cpd), compared to $0.9 \mathrm{cpd}$ in cat and $4 \mathrm{cpd}$ in primate. The spatial frequency bandwidth is 2.5 octaves, compared to 1.5 in cat and macaque. The median orientation tuning half-width is $28^{\circ}-29^{\circ}$, while in cats it is $19^{\circ}-24^{\circ}$ and in macaques $24^{\circ}$. In all species the tuning curve shows contrast invariance. Besides the decrease visual acuity, the largest differences in rodents is in the high fraction of simple cells in layer $2 / 3$ of the cortex, where $75 \%$ of the neurons are simple. In contrast, in macaque most of the cells outside layer 4 are complex. A second difference is that the percentage of direction tuned cells is lower in mice, with only $22 \%$ of cells having this property compared to more than $66 \%$ in carnivores and around $50 \%$ in primates. Another remarkable difference is that most inhibitory cells are unselective for orientation, while in cats the net inhibition a neuron receives was shown to be tuned [104]. Despite those differences the results show that even with the poor visual acuity, small eyes and a small V1 area, the primary visual cortex of mice has the ability to process the contours of the visual stimuli.

The functional features of single neurons in rodents are similar to primates and carnivores. What about their functional organization in space? Since the first recordings in mouse visual cortex it was shown that the spatial organization is not present $[102,105,106]$. This is also the case for rats $[107,108]$, hamsters [109], squirrels [110] and rabbits [111]. As shown in figure 1.4.1c), this lack of organization was finally confirmed at cellular level with 2photon calcium imaging [100], where the orientation of the neurons in labeled with colors. Due to the lack of a map in the tested rodents and lagomorphs this functional arrangement is commonly referred to as "Salt and Pepper layout". Since this name implies a white noise random spatial distribution of orientation preferences and this statistical property has not been measured experimentally, in this thesis the lack of organization will 
be referred to as "interspersed layouts".

\subsection{Previous approaches to understand the interspersed layout}

How can the neurons in rodents and lagomorphs be tuned for orientation in the absence of an orientation map? Or inversely, if neurons are tuned, why doesn't an orientation map develop? Neurons have a statistically higher probability of connecting with other neurons that are in their vicinity. In ordered layouts this means that neurons with similar tuning are wired together, establishing a coherent population response to a given stimulus. In interspersed layouts this is not the case, as many differently tuned neurons are close to each other. This difference lead to the idea that, although the preferred orientations are disordered in space, the interaction between the neurons might be highly specific, such that neurons with similar orientations are connected in disjoint sub-networks (reviewed in [112]). This "selective sub-network hypothesis" is consistent with a number of connectivity studies:

- Song et al. showed using quadruple recordings in layer 5 of rats that there is an increased representation compared to random of different three neuron connectivity motifs. They also showed that the strength of the synaptic weights are concentrated among few connections and that when a connection between two neurons is strong there is a higher chance of a reciprocal strong connection [113].

- This result is expanded by the results of Yoshimura et al. [114]. They performed paired recordings of excitatory neurons in layer $2 / 3$ in rats and evoked localized activity by photostimulation in different regions of all layers. Using cross correlation analysis of the activity evoked by the photostimulation they showed that if the tested neurons were connected together they shared the same input from layer 4 and within layer 2/3. On the other hand, excitatory input from layer 5 and inhibitory inputs from layer $2 / 3$ and 4 were shared by all neurons.

- The functional properties of this non-random connectivity was tested by Ko et al. in mice [115]. They used 2-photon calcium imaging to measure the orientation preference of neurons in the cortex and then performed electrical recording in acute slices to quantify the connection probabilities between the measured neurons. They found that neurons with similar preferred orientation or responding similarly to naturalistic stimuli have twice the probability of being connected.

- The question of how those sub-networks of orientation preference develop was addressed independently by Otsuki et al. [116] and Li et al. [117]. Both studies start from the finding that clonally related neurons that migrate through the radial glial fibers from a progenitor cell in the ventricular zone maintain a higher connection probability among them [118]. Using 2-photon calcium imaging the studies find that sister neurons exhibit a tendency towards similar orientation tuning with higher probability than random. The studies differ methodologically with respect to the age of the animals and the implementation of a transgenic type of mouse to label clonally related cells in the first and retroviral vector injection in the second, which might explain the quantitative difference in their finding. Li et al. also find that electrical gap junctions between the sister cells are necessary to increase the probability of sharing the same orientation tuning beyond chance level, as gap-junction blockers remove this effect. 
The "selective sub-networks hypothesis" tries to close the gap between maps and interspersed patterns. Although the results of those studies are congruent with this theory, it is very clear that connection-wise there are large differences between rodent and carnivore visual cortex, specially in the selectivity of inhibitory interactions:

- As already found by Yoshimura et al. in [114] and supported by a later study by the same group [119], adapting inhibitory cells connect to all pairs of measured neurons independent if the pair is connected to each other or not.

- With respect to functional synaptic connectivity, Hofer et al. showed using the same approach as Ko. et al that, distinct from excitatory neurons, inhibitory fast spiking PV positive neurons connect and also receive connections densely from all cells independent of their orientation preference [120].

- A similar approach to link function and connectivity of inhibitory cells was performed by Bock et al. [121]. 2-photon calcium imaging was used to quantify the orientation preference of the neurons and the underlying connectivity was reconstructed with large scale electron microscopy of thin sections. They also found that inhibitory neurons received convergent input from excitatory neurons from a broad range of orientation preferences.

- In maps, the long-range interactions are orientation specific, clustering in columns of similar orientation $[39,76,78,79,122]$. Using latex microsphere injections to trace the axons of neurons Van Hooser et al. showed that in gray squirrels there is no clustering of long-range connections [123], marking another difference in the wiring diagram of rodents.

The result of other studies question the degree of selectivity in the connections and the influence they have on the tuning of neurons:

- Using the same methods as in the previous paper, Ko et al. showed that the preferential connection between excitatory neurons of the same orientation preference is not present at eye opening but is acquired during development through activity-dependent plasticity mechanisms [124].

- Although neurons with similar orientation have a higher probability of being connected, this doesn't mean that the afferent inputs to one neuron come from cells with a restricted range of orientation preferences. This issue was addressed by Jia et al. [125] using high speed 2photon imaging combined with electrophysiological recordings to detect sub-threshold calcium hotspots in dendrites. The hotspots come from the corresponding input synapses and share the same functional tuning as the connected cell. By matching the hotspots with the presented visual stimuli the authors found that neurons receive inputs from cells of all kinds of preferred orientations, and that the oriented inputs are interspersed in the dendrites of the neuron.

- The previous finding lead to a comment by Priebe et al. [126] stating that the results make the rules of Hebbian plasticity appear unnecessary, since neurons in rodent visual cortex apparently wire together if they fire together or not. That indeed there is a large degree of plasticity in the rodent cortex, even without external manipulations like dark-rearing, was found by Wang et al. [127]. They showed that when orientation selectivity is developed in binocular neurons the orientation of the receptive fields of the left and right eye are not matched. Only through 
visual experience during maturation the matching of the binocular orientation preference is achieved. This findings demonstrate that neurons change their preferred orientation during development, meaning that the underlying orientation selective sub-networks would have to change and integrate.

- Finally, the idea that specific interactions between the neurons are needed to maintain orientation selectivity was refuted in a theoretical study by Hansel and Van Vreeswijk [128]. They found that in a network model with weakly tuned input to a balanced recurrent random network, neurons were well tuned and orientation selective. Selectivity is possible as untuned components of the input to each neuron caused by the random connections are canceled by inhibition.

The presented results don't contradict the idea that preferential connections within sub-networks exist, but make it very unlikely that rodent visual cortex exhibits an essentially identical neuronal circuit as primates as carnivores. In addition, they question their relevance in the formation and maintenance of the disordered orientation layout. A different approach is not to search similarities between the two kinds of functional organizations, but treat them as two separate collective phenomena with different properties. The main mechanisms of pattern formation in cortical networks might be shared, but differences between the species might lead either to the formation of maps or interspersed layouts. Indeed, the interspersed organization in rodents is not just observed in the primary visual cortex:

- The classical example for columnar organization in rodents is the so called barrel cortex, where the touch sensors of in the different facial whiskers are mapped to disjoint areas in the primary somatosensory cortex maintaining the relative positioning of the vibrissa [129]. But this organization is equivalent to the retinotopy in the visual cortex. Comparable to orientation selectivity in the visual cortex is the tuning for the movement direction of the vibrissa the neuron is encoding. Although an earlier electrophysiology study by Andermann et al. [130] found evidence of a vibrissa movement direction map in the barrel cortex of rats, using 2-photon calcium imaging and sampling from a larger set of neurons Kerr et al. and Sato et al. independently reported a high degree of disorder in the functional properties of the neurons [131, 132]. This result was further corroborated by Kremer et al. [133]. They reported that the organization of movement direction selective cells develops until long after the classical critical period and that there is a large degree of disorder until adulthood. Only after pooling from different animals and implementing strong spatial filters a map could be extracted.

- The primary auditory cortex is known to display a tonotopic organization, where there is a systematic progression between the represented frequencies [? ]. Using 2-photon calcium imaging two independent studies in mice by Rotshild et al. [134] and Bandyopadhyay et al. [135] showed that although a large scale tonotopy exists, there are high levels of heterogeneity in the represented frequencies and the intensity tuning is not spatially organized at all. Sub-threshold potential measurements show that although neighboring neurons share a common input they can have very different functional responses. This type of scatter at the single neuron level is also found in the retinotopy of rodent visual cortex, as described above [136].

What is the fundamental difference between the species that lead to the formation of orientation maps or interspersed patterns? Although the visual pathway has many conserved features in all 
mammals, rodents and lagomorphs are different to other species in many aspects:

- Structurally, Hustler et al. found that the pyramidal cell layers in rodents are more than half as small than in primates, even when controlled for brain size [137]. A study by Herculano-Houzel et al. show that rodent brains vary in mass as a power function of the number of neurons with exponent 1.5 while in primates it increases linearly [138]. This means that with equal size of the brain rodents would have a smaller number of neurons with a less dense neuronal packing compared to primates.

- Functionally there are also many differences. As discussed above, there is evidence that orientation selectivity emerges earlier in the visual pathway of rodents and lagomorphs compared to other species, where the percentage of orientation selective cells in the visual pathway before the visual cortex is higher [91]. In a study in mouse by Rochefort et al. it was found that direction selectivity develops at eye opening even in dark reared animals, and only later in development the number of orientation selective cells increases to become the most abundant in the cortex [139]. In comparison the ferret needs visual experience to develop direction selectivity [140].

A simple feature that distinguishes almost all studied rodents and lagomorphs from the well characterized primates, carnivores and scadentia is the size of the visual cortex. The primary visual cortex of the tested animals with interspersed layout might be too small to fit an organized orientation map. Although this is a common argument in discussions, the real role of the area size for functional organization has never been characterized. A counterargument to a potential role of area size was given by Van Hooser et al. [110]. They showed that the gray squirrel, a highly visual rodent with a visual cortex size comparable to ferrets and a visual acuity comparable to tree shrews, lacks ordered orientation maps. In chapter 5 of this thesis the role of V1 size in determining the organization of the layout will be analyzed in a optimization model.

With the exception of the work by Hansel and Van Vreeswijk, all the mentioned approaches above are experimental. What can theory teach us about the emergence of the different types of functional organizations? Can a model explain why some animals develop a map and others not? Until now there are only two theoretical models that can generate both ordered maps and interspersed layouts:

- Koulakov et al. implemented an optimization model to explore the functional organization a pattern must have to minimize the wiring costs for a given connectivity function [64]. When strict non-selective connections between the neurons are imposed, i.e. the neuron has to connect with equal amounts of neurons for all orientations, interspersed patterns are the most cost effective. With increasing selectivity, i.e. a higher number of connections with neurons with similar orientation preference, the most cost effective layout becomes periodic.

- Paik et al. proposed a model based on the segregated roughly hexagonal mosaic formed by ON and OFF RGCs in the retina [141,142]. According to the model, when neurons in V1 sample from the overlaid ON and OFF mosaics a Moiré interference pattern is generated. ON-OFF dipoles emerge in the receptive field of cortical neurons, seeding their orientation preference and forming an ordered periodic map. Depending on the relative orientation of the mosaics and their size, the wavenumber of the pattern increases and it becomes qualitatively disorganized due to the very short wavelength. Although very simple, many aspects of this model have been 
criticized. RGC mosaics in the retina don't have the characteristics that model needs, lacking a typical scale and long-range correlations [143]. The model predicts that this mechanism leaves an hexagonal footprint in the map, but its existence is difficult to test experimentally [144] and can also be generated by many self-organization models [41, 42].

It is important to underline that these two models are not dynamical. A structure in the intra-cortical interactions or in the RGC positions is assumed and the pattern that satisfies the conditions is directly calculated. After 40 years of dynamical models for pattern formation none was ever reported to exhibit a transition to interspersed patterns with a change of parameters. Determining whether such a model exists could be very informative, because it might lead to a clarification of what is the fundamental difference in the mechanism that leads to the formation of interspersed and ordered patterns.

\subsection{Overview of this work}

In this thesis I will introduce a class of models and an optimization approach that can generate both types of functional organization found in the primary visual cortex of mammals: ordered maps with the invariant layout statistics found in primates and carnivores and interspersed arrangements. The conditions on the intra-cortical interaction for the generation of interspersed layouts are analytically calculated and confirmed in a biologically detailed model. The model is further expanded to include representations to both eyes and to modulate the degree of plasticity during the critical period. The generated interspersed pattern is characterized both structurally and dynamically and an optimization theory is proposed modeling a V1 size dependent transition between the different layout types. The thesis is organized as follows:

In the second chapter of the thesis the dynamical models for pattern formation are introduced. First the model proposed by Wolf $[21,22]$ is generalized by describing all intra-cortical interactions as convolutions. It is shown numerically that depending on the parameters of the interaction kernels the model can generate both types of layouts. The interspersed pattern is generated dynamically, and is not a consequence of the randomness of the initial condition. With the adequate interaction parameters, starting the simulations from ordered maps or from an imposed iso-orientation domain lead to the dynamical disorganization of the layout. Secondly, to understand the underlying principles leading to interspersed patterns, the model is solved in a one dimensional cortex. It is shown that ordered maps exist over wide regions of parameter space as stationary solutions. An analytical expression for their stability is calculated. The parameters of the interaction kernels are reduced using experimental data and the predicted stability diagram of the map solutions are calculated. This leads to the main result of the thesis: with strong local inhibition all ordered stationary solutions become unstable, leading to the development of an interspersed pattern. The generality of this finding is tested in a well established correlation-based model for pattern formation [53]. This model is chosen because its assumptions are closely tied to biology. It is shown that the conclusions of the abstract model apply also to this biologically detailed model, meaning that with strong inhibition the neurons develop orientation selectivity but preferred orientations remain interspersed in space.

The third chapter of the thesis deals with an expansion of the model to include the tuning to binocular stimuli. The experimental results obtained by Wang et al. [127] discussed above about the late matching of binocular receptive fields show that orientation preference is very plastic, which is 
an argument for dynamical models like the one proposed, but poses questions of how the neurons can specifically match their response while embedded in a disordered network. In this chapter first inter-eye coupling terms are included in the model by examining if the resulting dynamics satisfy a set of coupling symmetry conditions. Next the dynamical equations are solved for non-interacting neurons to find the coupling parameter regime where the binocular orientation is matched after the earlier emergence of orientation selectivity. Once this regime is found, strong inhibitory lateral interaction between the neurons are included and it is numerically shown that the interspersed pattern emerges and is maintained while the neurons match their orientation preference. This shows that the interspersed pattern can be understood as a fundamental consequence of strong inhibition and can coexist with other mechanisms that increase the order of receptive field organization. This chapter also describes how to include changes in the degree of plasticity found during the critical period in the model. Numerical analyzes are used to predict the consequences in binocular matching in a PSD95 knock-out mutant, where the critical period is prolonged but the orientation selectivity is reduced. The simulations predict that even with an extended critical period the degree of binocular orientation matching is decreased.

In the fourth chapter the interspersed pattern obtained in the simulations are characterized in detail. The first part deals with the dynamical characterization. It is shown that the disordered arrangement is caused by the mutual repulsion of the optimal orientation for each neuron. This leads to frustration and glassy dynamics, with a power law decrease in the rates of changes in the orientation of the neurons. Next the energy landscape of solutions in the model is addressed. In the interspersed regime the number of solutions is very large and the energy barriers between them are low, making the system more susceptible to noise. This result is corroborated with numerical simulations of stochastic differential equations. It is also shown in these simulations that freezing the dynamics of a fraction of neurons in maps leads to a large decrease in the number of available states, radically decreasing the "diffusion" of preferred orientations in the presence of noise. In comparison, even after freezing the dynamics of a large fraction of neurons in interspersed patterns, the number of available states is still very high, such that the diffusion coefficient in noisy simulations remains essentially constant. The second part of this chapter deals with the structural characterization of the interspersed pattern. It is shown that the final layout is not random, but that the preferred orientations between the neighboring neurons are negatively correlated. The magnitude of this correlation depends on the range of the inhibitory interaction and the number of neurons in this range. As more neurons interact with each other this correlation is decreased, making it a difficult prediction to test experimentally. On the other hand, it is shown that random patterns with the same correlation structure are not solutions in the system. Two different structural measures that are sensitive to phase randomization are introduced, the discrepancy and the Swindale coverage. The first measures the homogeneity of the disorder for any pattern interval. The second measures the homogeneity of the representation of the pattern for any possible oriented stimulus in space. Interspersed patterns have a better discrepancy and coverage compared to random layouts with and without negative correlations. Finally an stimulus driven optimization model is presented showing that interspersed patterns have an optimal stimulus coverage [145].

In the fifth chapter of the thesis the question of why some species have maps and others interspersed patterns is approached from an optimization perspective. Since the size of the hypercolumn remains roughly constant in all species with maps, an increase in the size of V1 leads to an increase in the number of hypercolumns representing an area in visual space. The consequence of this is that with increasing cortex size the difference in the coverage between interspersed patterns and ordered maps 
is reduced. On the other hand, following the results and considerations of Koulakov and Chklovskii [64], the wiring costs of the different layouts are calculated for increasing cortex size assuming a orientation selective interaction as found in mouse visual cortex [115]. Since specific wiring costs depends only on the immediate vicinity of neurons and with constant hypercolumn size do not change with cortex area, both costs remain roughly constant and are always lower for ordered maps. In an optimization model that combines both coverage and wiring cost a transition between the different layouts for increasing cortex size is predicted.

In the sixth chapter of the thesis the prediction of the lability of preferred orientations in the rodent visual cortex is tested experimentally. In collaboration with the Max Planck Institute for Biological Cybernetics in Tübingen, 2-photon calcium imaging of the evoked activity by visual stimulation in the rat visual cortex is performed. The aim of the first part of the chapter is to search for a stimulation and tuning curve estimation paradigm that lead to small confidence intervals of the estimated orientation preference, such that significant changes in time of the preferred orientation of the neurons can be detected. Two stimulation paradigms are tested: episodic stimulation, where drifting high contrast gratings with different orientations are presented in discrete intervals, and continuous stimulation, where the transition between the drifting orientation is obtained without interruption by the rotation of the grating [146]. Three different estimation methods are compared: vector average, Levenberg-Marquardt and Bayesian inference [147]. The results show that within a stimulation paradigm the difference in the estimated preferred orientation between the methods is not significant, but that in Bayesian inference the confidence intervals are smaller and decrease faster in size with the number of stimulus sets presented. The distribution of estimated orientations in episodic stimulation was more reliable than with continuous stimulation, and is therefore chosen for the second part of the chapter. There the data of a recording is divided in parts and the tuning curve is estimated in each one of them. In accordance to the prediction of the model, this approach shows that the preferred orientation of at least $20 \%$ of the neurons changes significantly during the experiment in less than half and hour.

In the last chapter the results of the thesis and future directions for the study of interspersed patterns are discussed. 


\section{Chapter 2}

\section{Understanding the formation of interspersed layouts in visual cortical networks}

The approach proposed by Wolf [22] described in the introduction is the first model for orientation preference map development that shows analytically the existence and stability of quasi-periodic patterns that quantitatively resemble the maps measured in the experiments. On the other hand, when the aim is to understand the formation of the interspersed arrangements of orientation preferences from rodents and lagomorphs the model falls short. The analytical treatment of the model is based on the imposition of a critical circle by the linear term, defining the periodicity of the pattern. The nonlinear analysis then determines how to make many modes stable on this critical circle. In rodents the preferred orientation of the neurons are disorganized in space, such that a general periodicity of the pattern is not present. The linear functional can be changed to remove this constrain, but this also means that the weakly nonlinear analysis used in the amplitude equations would have to be strongly modified since the values of $\left|\mathbf{k}_{j}\right|$ are not bounded to be close to $k_{c}$.

In this chapter the long-range interaction model is generalized such that disorganized layouts are not excluded by construction but can form in principle. For this all intra-cortical interactions are described as convolutions. As in the previous model the symmetries of the system span a subspace of spatially ordered solutions, and using perturbation theory on a one dimensional cortex equations for their stationarity and stability are derived for a general interaction shape. After describing a family of intra-cortical interaction shapes that are congruent to experimental observations in rodents with three free parameters, the derived equations are used to test the stability of different ordered solutions. It is found that local strong inhibition makes all existing stationary solutions unstable, such that the arrangement of orientations is disordered. The analytical results from the stability analysis are tested by numerical simulations of the dynamical equation. The interspersed layout is dynamically generated in the model, such that ordered initial conditions like maps are disordered in time. At the end of this chapter the condition of strong inhibition in generating disordered patterns is successfully tested in a biologically detailed model based on Hebbian competition in the LGN-V1 synapses [53]. 


\subsection{Generalization of the model}

The generalized model splits from the previous approach after the symmetry conditions for $\hat{F}$ are stated. Here the lateral interactions between the neurons play a central role from the beginning of the definitions

$$
\tau_{z} \frac{\partial z_{i}}{\partial t}=\hat{F}\left[z, w_{i, j}\right]
$$

where $w_{i j}$ weights the strength of the interaction between neuron $i$ and $j$. Due to translation and rotation symmetry the spatial component of the interaction between the neurons can only be distance dependent. The interaction strength between the neurons $w_{i j}$ is also a dynamical variable, representing the changes in synaptic connections. It approaches with time constant $\tau_{w}$ the value $w_{i j}^{\infty}$, which depends on the position of the neurons $\mathbf{x}_{i}$ and $\mathbf{x}_{j}$ and their corresponding tuning $z_{i}$ and $z_{j}$.

$$
\tau_{w} \frac{\partial w_{i, j}}{\partial t}=w_{i, j}^{\infty}\left(\mathbf{x}_{i}-\mathbf{x}_{j} \mid z_{i}, z_{j}\right)-w_{i, j}(t)
$$

A neuron $i$ has to change its interactions $w_{i j}$ to neighboring neurons in order to change its tuning. Focusing on the timescale of orientation dynamics, one can assume that when there is a change in orientation the needed changes in the interactions have already been made, such that $\tau_{z} \gg \tau_{w}$. Therefore, the interaction dynamics can be tied to the tuning dynamics by directly setting $w_{i, j} \rightarrow w_{i, j}^{\infty}$ and using

$$
\frac{\partial z_{i}}{\partial t}=\hat{F}\left[z, w_{i, j}^{\infty}\right]
$$

\subsubsection{Dynamical equation}

Instead of treating the linear and nonlinear part of equation 2.1.1 separately, in this approach an example equation for $\hat{F}$ that satisfies the symmetry conditions will be directly given. Remember that this proposed equation, although based on them, is not meant to delineate the biological mechanisms found in the brain, but to give an analytically tractable example of the same universal class of functions sharing many dynamical properties with them. The equation to use is

$$
\tau_{z} \frac{\partial z_{i}}{\partial t}=\tilde{r} z_{i}-z_{i}\left|z_{i}\right|^{2}+\frac{1}{N} \sum_{j}^{N}\left(z_{j}-z_{i}\right) w_{i, j}^{\infty}\left(\mathbf{x}_{i}-\mathbf{x}_{j} \mid z_{i}, z_{j}\right)
$$

Notice that in this approach the index of individual neurons $i$ is kept, since a continuous orientation field $z$ is not assumed. The first two terms show that, in the absence of intracortical interactions, with $\tilde{r}>0$ the selectivity of the neurons increases and saturates at $A_{i}=\sqrt{\tilde{r}}$. This is represented in figure 2.1.1a), where the saturation of the color encodes the selectivity of the neuron. The third term shows that the tuning of neuron $i$ will be pulled toward the tuning of all neurons $j$ in its vicinity depending on the strength of interaction $w_{i, j}^{\infty}$ between them, as represented in figure 2.1.1b), where the hue of the color encodes the preferred orientation of the neuron. Only the third term can change the preferred orientation of the neurons, such that the patterns that will be solutions to this equation will depend on the choice of the interaction $w_{i, j}^{\infty}$. 


\subsubsection{Describing the interactions}

The interaction is split in two parts, one that only depends on the distance between the neurons and a second that also scales with selectivity $\sigma_{z}$ depending on the similarity of the tuning

$$
w_{i, j}^{\infty}\left(\mathbf{x}_{i}-\mathbf{x}_{j} \mid z_{i}, z_{j}\right)=K\left(\mathbf{x}_{i}-\mathbf{x}_{j}\right)+K_{o s}\left(\mathbf{x}_{i}-\mathbf{x}_{j}\right) e^{-\frac{\left|z_{j}-z_{i}\right|^{2}}{\sigma_{z}^{2}}}
$$

For concreteness each interaction kernel is expressed as a sum of Gaussian functions, representing excitation and inhibition

$$
\begin{gathered}
K\left(\mathbf{x}_{i}-\mathbf{x}_{j}\right)=\frac{E}{2 \pi \sigma^{2}} e^{-\frac{\left(\mathbf{x}_{i}-\mathbf{x}_{j}\right)^{2}}{2 \sigma^{2}}}-\frac{I}{2 \pi \varrho^{2}} e^{-\frac{\left(\mathbf{x}_{i}-\mathbf{x}_{j}\right)^{2}}{2 \varrho^{2}}} \\
K_{o S}\left(\mathbf{x}_{i}-\mathbf{x}_{j}\right)=\frac{E_{O S}}{2 \pi \sigma_{o s}^{2}} e^{-\frac{\left(\mathbf{x}_{i}-\mathbf{x}_{j}\right)^{2}}{2 \sigma_{o s}^{2}}}-\frac{I_{O S}}{2 \pi \varrho_{o s}^{2}} e^{-\frac{\left(\mathbf{x}_{i}-\mathbf{x}_{j}\right)^{2}}{2 \varrho_{o s}^{2}}}
\end{gathered}
$$

$E$ and $\sigma$ are the strength and range of unselective excitation, and $I$ and $\varrho$ the strength and range of unselective inhibition. The same parameters are present for the selective interaction. For the analytical and numerical calculations the following approximation will be used: if the selectivity of the selective interactions is of the same order as the selectivity of the neurons, $\sigma_{z} \sim \mathcal{O}(z)$, one can use

$$
K_{o s}\left(\mathbf{x}_{i}-\mathbf{x}_{j}\right) e^{-\frac{\left|z_{j}-z_{i}\right|^{2}}{\sigma_{z}^{2}}} \simeq K_{o s}\left(\mathbf{x}_{i}-\mathbf{x}_{j}\right)\left(1-\frac{\left|z_{i}-z_{j}\right|^{2}}{\sigma_{z}^{2}}\right)
$$

Inserting this in the dynamical equation 2.1.2 and expanding leads to

$$
\begin{aligned}
\tau_{z} \frac{\partial z_{i}}{\partial t}= & \tilde{r} z_{i}-z_{i}\left|z_{i}\right|^{2}+\frac{1}{N} \sum_{j}^{N}\left(z_{j}-z_{i}\right)\left(K\left(\mathbf{x}_{i}-\mathbf{x}_{j}\right)+K_{o s}\left(\mathbf{x}_{i}-\mathbf{x}_{j}\right)\left(1-\frac{\left|z_{i}-z_{j}\right|^{2}}{\sigma_{z}^{2}}\right)\right) \\
= & \tilde{r} z_{i}-\left(1+\frac{\widetilde{K}_{o s}(0)}{\sigma_{z}^{2}}\right) z_{i}\left|z_{i}\right|^{2}+\frac{1}{N} \sum_{j}^{N}\left(K\left(\mathbf{x}_{i}-\mathbf{x}_{j}\right)+K_{o s}\left(\mathbf{x}_{i}-\mathbf{x}_{j}\right)\right) z_{j} \\
& -\frac{1}{N \sigma_{z}^{2}} \sum_{j}\left(K_{o s}\left(\mathbf{x}_{i}-\mathbf{x}_{j}\right)\left(-z_{i}^{2} \overline{z_{j}}-2\left|z_{i}\right|^{2} z_{j}+2 z_{i}\left|z_{j}\right|^{2}+\overline{z_{i}} z_{j}^{2}-z_{j}\left|z_{j}\right|^{2}\right)\right)
\end{aligned}
$$

where $\widetilde{K}_{o s}(0)$ is the Fourier transform of $K_{o s}$ evaluated at zero and all resulting constants multiplying $z_{i}$ have been included in $\tilde{r}$. If the unselective interaction $K\left(\mathbf{x}_{i}-\mathbf{x}_{j}\right)$ has an unique maximum $k_{c}$ in Fourier space, the linear part of this general equation has the same properties of the functional $\hat{L}[z]$ of the original model in equation 1.3.5. In this case also a critical circle of size $k_{c}$ in the power spectrum of the pattern is imposed, and the non-linear interactions defined by the orientation selective terms will determine how many modes can coexist inside this circle. Therefore, for a specific choice of parameters of the interaction kernels the general model can be mapped to the original model and exactly the same amplitude equations are obtained by weakly nonlinear analysis, demonstrating an identical set of solutions. 


\subsubsection{The model generates ordered and disordered layouts}

Equation 2.1.4 can be solved numerically for different choices of the interaction parameters and the layout that emerges from the system can be checked. The results are shown in figure 2.1.1c) to f). The modeled system is composed of a grid of 128x128 equally spaced neurons with periodic boundary conditions. The integration of the function was done using a 4th-5th order Runge-Kutta-Fehlberg scheme where the error was kept below $0.01 \tilde{r}$, where $\widetilde{r}=0.1$. The simulation time frame is defined by $\tau=\frac{1}{\tilde{r}}$, which is the time-scale of the initial emergence of orientation selectivity. All simulations are done without adding noise to underline the role of the interaction on the dynamics pattern formation (the effect of adding noise is shown in chapter 4). The figures show only a region of the simulated pattern. It is important to stress that the results of the simulations remain valid for a large range of parameters. Their exact relation to the emerging layout will be examined below.

In figure 2.1.1c) the initial condition are randomly orientation selective neurons with low selectivity $z=\frac{1}{100} \sqrt{\tilde{r} \xi_{1}} e^{i 2 \xi_{2}}$, where $\xi_{1} \in \mathcal{U}[0,1]$ and $\xi_{2} \in \mathcal{U}[0, \pi]$ are are sequences of uniformly distributed random numbers. The parameters of the interaction are chosen to resemble the model by Wolf. The unselective kernel is a Mexican hat that imposes a fixed wavenumber to the solution. The size of the simulated box is scaled to fit 10 hypercolumns $\Lambda$ in the system, $L=10 \Lambda$. The orientation selective interactions are long-ranged, as was shown to be required to have a stable pinwheel-rich solution. The range of the later was scaled to be twice the size of an hypercolumn. Defining $\Lambda=2 \pi$, the chosen parameters are

$$
E=4.50 \sigma=1.08 \quad I=5.50 \quad \varrho=2.01 \quad E_{o S}=0 \quad I_{o s}=1 \quad \varrho_{o s}=12.57
$$

The profile of the interactions is depicted next to the initial condition, where unselective interactions are filled with gray and orientation selective interactions are marked with a dashed line. The results at $1 \tau$ and $100 \tau$ show that, given the long-range selective interactions, a quasi-periodic map emerges as stable solution to the model. This shows that this generalization of the model can reproduce the results from the original model.

In figure 2.1.1d) the same initial condition and scaling of the simulated box are used as in c). In the rodent visual cortex only excitatory interactions are orientation selective, while inhibitory neurons connect with their neighbors independent of their tuning [115]. Therefore $I_{o s}=0$ is set. There are no long-range patchy connections in the rodent visual cortex, meaning that the selective and unselective excitatory interactions have the same range [123]. This makes $\sigma_{o s}=\sigma$. For the unselective interaction the relative range of inhibition is decreased, incrementing the amount of local inhibition and inverting the Mexican hat. The parameters used are

$$
E=4.50 \quad \sigma=1.70 \quad I=5.50 \varrho=1.39 \quad E_{o s}=0.06 \quad \sigma_{o s}=1.39 \quad I_{o s}=0
$$

Using these conditions, the results in d) show that orientation selectivity increases in the system and saturates, following a), but that no ordered arrangement of orientations emerges as solution. The layout is disorganized and remains like this for simulations of $100 \tau$ and longer. This shows that for a different choice of parameters the model can also generate interspersed patterns as found in rodents and lagomorphs.

To show that the disordered layout is generated by the dynamics and is not a direct consequence of the random initial condition, in figure 2.1.1e) the pattern in c) at $100 \tau$ with lowered selectivity is 
a

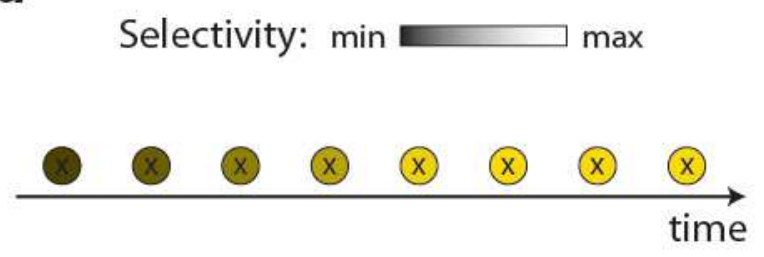

b

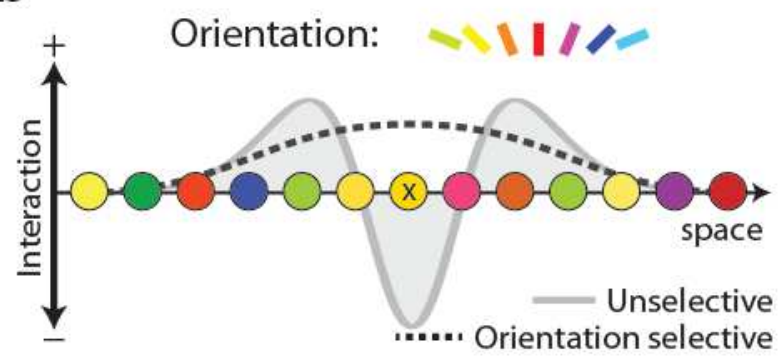

C Interaction profile

Initial condition

$t=5 \tau$ $t=100 \tau$

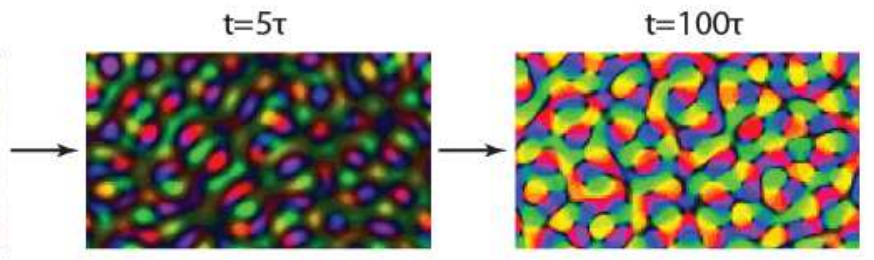

d
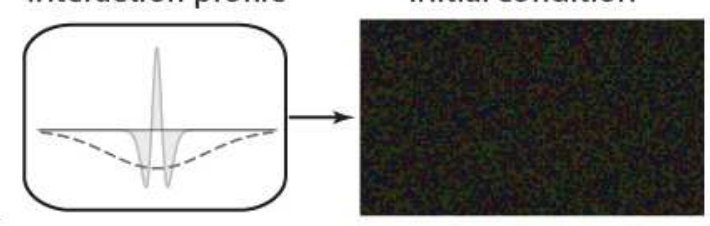

$\rightarrow$
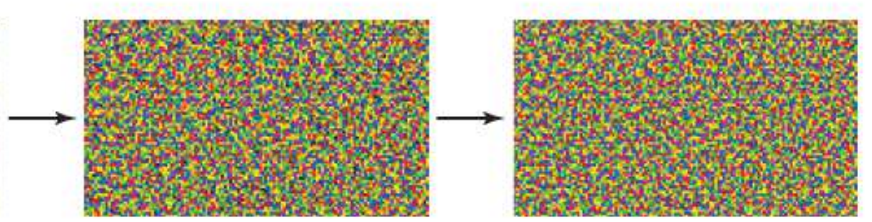

e
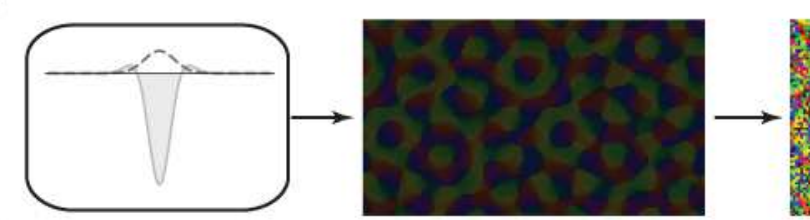

f
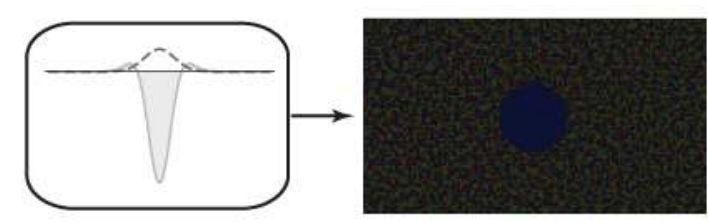

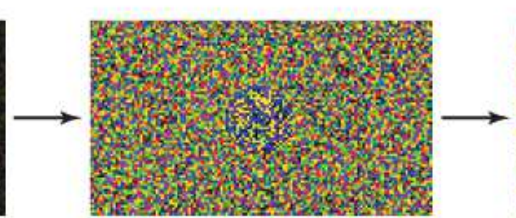

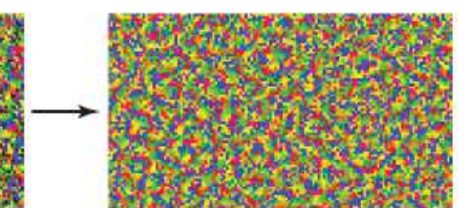

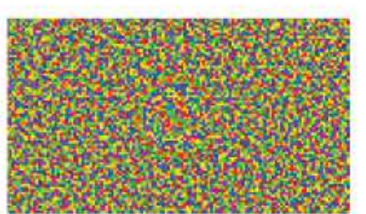

Figure 2.1.1: Explanation of the general model and simulation results. a) Neurons in the model increase and saturate their selectivity, represented by the brightness of the color. b) Neurons interact through orientation selective and unselective interactions with their neighbors, where orientation is labeled with colors. c) to f) Simulation of the model for different interaction parameters. c) With Mexican hat shaped unselective interactions and long-range orientation selective interactions the system develops from a random unselective state into a quasi-periodic map of orientation preferences as in the long-range interaction model. e) With increased local inhibition, selectivity develops in the neurons but the layout remains disorganized. e) Using the final pattern in c) as initial condition shows that the disordered layout is obtained dynamically and is not a consequence of the random initial conditions. f) The final layout of d) with an imposed column of equally tuned neurons is used as initial condition. The ordered imposed by the orientation column is also disrupted by the dynamics of the system. 
used as initial condition. As the system develops, this organization is lost and a disordered pattern emerges. A small resemblance to the initial condition can still be detected, but this is lowered for longer simulations or totally removed with a very small amount of noise in the simulations (see chapter 4).

To further show that the dynamics actively disorganize the system, in figure 2.1.1f) the layout of d) at $100 \tau$ is used with decreased selectivity as initial condition, where a column of equally tuned neurons is imposed. Also in this case the system reorganizes into an interspersed pattern and the imposed column is lost.

Taken together these results show that, depending on the choice of interaction parameters, this model can generate both types of organizations found in nature. In the next section the role of the interaction kernels in the formation of the different layouts will be analyzed further. For this purpose an analytical treatment of the equation in a one dimensional cortex is performed, where the solutions can be obtained and their stability analytically calculated. The conclusions of the one dimensional model are directly applicable to two dimensional systems as shown in subsequent simulations.

\subsection{Analytical solution of the model in 1D}

The simulations presented in section 2.1.3 show that depending on the interaction parameters ordered maps or disordered arrangements of orientation preference are generated in the model. Is the transition between the different layout types gradual or sharp? What are the essential conditions for each layout type to emerge? In this section the stationarity of orientation patterns that are invariant under the dynamics is analyzed. Then their stability and how it changes depending on the interaction parameters is calculated. To be able to solve the equations analytically, the model will be examined in a one dimension, where ordered patterns and disordered arrangements can still be identified. The stability conditions developed here will be used in the next section to show an interaction parameter regime where selectivity increases but none of the stationary patterns are stable, making a disordered arrangement of orientation preference the only solution.

\subsubsection{Stationary solutions}

The symmetries of the system (equations 1.3.1 to 1.3.4) imply an invariant subspace of spatially ordered solutions of the form $z_{i}=A(t) e^{i k x_{i}}$, where $k$ is the wavenumber of the pattern. This include three different types of stationary solutions:

- Unselective state, with $A=0$.

- Perfectly periodic state, with $A>0$ and $k \neq 0$.

- Uniformly selective state, where $A>0$ and $k=0$.

For each solution the conditions on the interaction parameters for its existence will be calculated. 


\section{Unselective state}

It is easy to see that $\hat{F}[0]=0$, such that $A=0$ is always a stationary solution independent of the choice of interaction parameters. This represents the unselective state the neurons are during the first moments of cortical development.

\section{Perfectly periodic state}

Inserting $z_{i}(t)=A(t) e^{i k x_{i}}$ in equation 2.1.4 leads to

$$
\begin{aligned}
\hat{F}\left[A(t) e^{i k x_{i}}\right]= & \tilde{r} A(t) e^{i k x_{i}}+\frac{1}{N} \sum_{j}^{N}\left(K\left(x_{i}-x_{j}\right)+K_{o s}\left(x_{i}-x_{j}\right)\right) A(t) e^{i k x_{j}} \\
& -\left(1+\frac{\widetilde{K}_{o s}(0)}{\sigma_{z}^{2}}\right) A(t) e^{i k x_{i}}|A(t)|^{2} \\
& -\frac{A(t)|A(t)|^{2}}{N \sigma_{z}^{2}} \sum_{j}\left(K_{o s}\left(x_{i}-x_{j}\right)\left(2 e^{i k x_{i}}-3 e^{i k x_{j}}-e^{i k\left(2 x_{i}-x_{j}\right)}+e^{i k\left(2 x_{j}-x_{i}\right)}\right)\right)
\end{aligned}
$$

Using the definition of the Fourier transform, the interaction term can be calculated as

$$
\frac{1}{N} \sum_{j} K\left(x_{i}-x_{j}\right) e^{i k x_{j}}=e^{i k x_{i}} \widetilde{K}(k)
$$

where $\widetilde{K}(k)$ is the Fourier transform of $K\left(x_{i}-x_{j}\right)$. Using $\widetilde{K}(0)=\frac{1}{N} \sum_{j} K\left(x_{i}-x_{j}\right)$ leads to

$$
\hat{F}\left[A(t) e^{i k x_{i}}\right]=A(t) e^{i k x_{i}}\left[\tilde{r}+\widetilde{K}(k)+\widetilde{K}_{o s}(k)-|A(t)|^{2}\left(1+\frac{1}{\sigma_{z}^{2}}\left(3 \widetilde{K}_{o s}(0)+\widetilde{K}_{o s}(2 k)-4 \widetilde{K}_{o s}(k)\right)\right)\right]
$$

For the pattern to be stationary, either $A=0$ as in the unselective state, or

$$
\left|A_{k}\right|^{2}=\frac{\tilde{r}+\widetilde{K}(k)+\widetilde{K}_{o s}(k)}{1+\frac{1}{\sigma_{z}{ }^{2}}\left(3 \widetilde{K}_{o s}(0)+\widetilde{K}_{o s}(2 k)-4 \widetilde{K}_{o s}(k)\right)}
$$

The stationary selectivity depends on the choice of interaction kernels $K$ and $K_{o s}$ and the wavenumber of the pattern $k$. To ensure the existence of at least one stationary pattern with $A_{k}>0$ for all combination of interaction parameters, the bifurcation parameter $r$ is introduced:

- If the denominator in equation 2.2.2 is positive, the numerator has to be positive and $\tilde{r}$ is defined as

$$
\tilde{r}=r-\max \left(\widetilde{K}(k)+\widetilde{K}_{o s}(k)\right)
$$


- If the denominator in equation 2.2.2 is negative, the numerator has to be negative and $\tilde{r}$ is defined as

$$
\tilde{r}=-r-\min \left(\widetilde{K}(k)+\widetilde{K}_{o s}(k)\right)
$$

In both cases, when $r \leq 0$ the only stationary solution is $A=0$. When $r>0$ this state is unstable and symmetry is spontaneously broken and spatially periodic patterns become a stationary solution to the dynamics.

\section{Uniformly selective state}

This is a special case of the previous stationary solution, where $A_{k}$ for $k=0$ in equation 2.2.2 is positive. In this case all neurons are selective to the same orientation, as in the vertical axis of a columnar organization. The selectivity of this uniform state is given by

$$
|A|^{2}=\tilde{r}+\widetilde{K}(0)+\widetilde{K}_{o s}(0)
$$

\subsubsection{Stability of stationary solutions}

The stationary patterns have an uniform or progressively changing orientation preference with wave number $k$. This means that the tuning of each neuron only depends on its position in space, $z_{i} \rightarrow$ $z\left(x_{i}\right)$. Therefore the limit $N \rightarrow \infty$ can be taken to treat the cortex as a continuous system and calculate the interactions as integrals. Having an exact expression for the selectivity $A$ of the stationary patterns allows us to calculate the dynamics of a perturbation $\delta z$ upon them. In each case the interaction parameters determine the eigenvalue of the perturbation. If the eigenvalue is positive, then the pattern $A_{k} e^{i k x}$ will be unstable.

\section{Unselective state}

A small perturbation $\delta z$ upon the stationary solution $A=0$ is performed. The linearized dynamics of the perturbation is given by the linear component of $\hat{F}[\delta z]$

$$
\hat{L}_{0}[\delta z]=\tilde{r} \delta z+\frac{1}{N} \sum_{j}^{N}\left(K\left(x_{i}-x_{j}\right)+K_{o s}\left(x_{i}-x_{j}\right)\right) \delta z
$$

To check if ordered patterns emerge from the unselective state, the perturbation $\delta z_{i}=A e^{i k x_{i}}$ is inserted obtaining

$$
\hat{L}_{0}\left[A(t) e^{i k x_{i}}\right]=A(t) e^{i k x_{i}}\left[\tilde{r}+\widetilde{K}(k)+\widetilde{K}_{o s}(k)\right]+\mathcal{O}\left(\delta z^{3}\right)
$$

Therefore the growth rates of the perturbation $\lambda(k)$ are obtained from the Fourier transform of the linear component of $\hat{F}[z]$ 


$$
\lambda(k)=\tilde{r}+\widetilde{K}(k)+\widetilde{K}_{o s}(k)
$$

With the bifurcation parameter $r>0$ in the definition of $\tilde{r}$ in equations 2.2.3 or 2.2.4, $\lambda(k)$ allays crosses the zero line and gets positive for at least one $k$, making the homogenous solution $A=0$ unstable.

\section{Perfectly periodic state}

Examining the dynamics of a perturbation $\delta z$ upon the stationary solution $z=A_{k} e^{i k x}$ with $A_{k}>0$, the linearized dynamics is

$$
\begin{aligned}
\hat{L}_{k}[\delta z(x, t)]= & \tilde{r} \delta z+\int_{\mathbb{R}}\left(K(x-y)+K_{o s}(x-y)\right) \delta z(y, t) d y \\
& -A_{k}^{2}\left(2+\frac{4 \widetilde{K}_{o s}(0)}{\sigma_{z}^{2}}-\frac{4 \widetilde{K}_{o s}(k)}{\sigma_{z}^{2}}\right) \delta z(x, t) \\
& -A_{k}^{2}\left(1+\frac{\widetilde{K}_{o s}(0)}{\sigma_{z}^{2}}-\frac{2 \widetilde{K}_{o s}(k)}{\sigma_{z}^{2}}+\frac{\widetilde{K}_{o s}(2 k)}{\sigma_{z}^{2}}\right) e^{2 i k x} \overline{\delta z}(x, t) \\
& -A_{k}^{2} \int_{\mathbb{R}} \frac{K_{o s}(x-y)}{\sigma_{z}^{2}}\left(-4+2 e^{i k(x-y)}+2 e^{-i k(x-y)}\right) \delta z(y, t) d y \\
& -A_{k}^{2} \int_{\mathbb{R}} \frac{K_{o s}(x-y)}{\sigma_{z}^{2}}\left(2 e^{i k(x+y)}+e^{2 i k y}-e^{2 i k x}\right) \overline{\delta z(y, t)} d y+\mathcal{O}\left(\delta z^{3}\right)
\end{aligned}
$$

To find the eigenfunctions $\psi_{k}$ of this spatially periodic integro-differential operator the Floquet-Bloch theorem is used [75]. It states that the eigenfunctions have the same period of the operator up to a phase shift $Q$. The Bloch phase $Q$ plays the role of a perturbation parameter, labeling all possible Eckhaus instabilities of a stationary pattern in one dimension. Following this, the eigenfunctions have the form

$$
\psi_{k}^{Q}(x, t)=a_{+}(t) e^{i(k+Q) x}+a_{-}(t) e^{i(k-Q) x}
$$

For a given $Q, a_{+}$and $a_{-}$satisfy the following matrix equation

$$
\mu(k, Q)\left(\begin{array}{c}
a_{+}(t) \\
a_{-}(t)
\end{array}\right)=M_{2 \times 2}\left(\begin{array}{c}
a_{+}(t) \\
a_{-}(t)
\end{array}\right)
$$

Where $\mu$ are the eigenvalues of the operator, $\hat{L}_{k}\left[\psi_{k}^{Q}\right]=\mu(k, Q) \psi_{k}^{Q}$. Since this is an optimization model the matrix $M_{2 \times 2}$ will be symmetric, such that all eigenvalues are real. The entries of the matrix are found by inserting the eigenfunction $\psi_{k}^{Q}(x, t)$ into equation 2.2.7 and separating terms proportional to $a_{+}(t) e^{i(k+Q) x}, a_{-}(t) e^{i(k+Q) x}, a_{+}(t) e^{i(k-Q) x}$ and $a_{-}(t) e^{i(k-Q) x}$. To make the calculations more transparent, equation 2.2.7 is split by lines and treated separately

$$
\hat{L}_{k}[\cdot]=\hat{L}_{k}^{1}[\cdot]+\hat{L}_{k}^{2}[\cdot]+\hat{L}_{k}^{3}[\cdot]+\hat{L}_{k}^{4}[\cdot]+\hat{L}_{k}^{5}[\cdot]
$$




$$
\begin{aligned}
\hat{L}_{k}^{1}\left[\psi_{k}^{Q}\right]= & \tilde{r}\left(a_{+}(t) e^{i(k+Q) x}+a_{-}(t) e^{i(k-Q) x}\right) \\
& +\int_{\mathbb{R}}\left(K(x-y)+K_{o s}(x-y)\right)\left(a_{+}(t) e^{i(k+Q) y}+a_{-}(t) e^{i(k-Q) y}\right) d y \\
= & \left(\tilde{r}+\widetilde{K}(k+Q)+\widetilde{K}_{o s}(k+Q)\right) a_{+}(t) e^{i(k+Q) x} \\
& +\left(\tilde{r}+\widetilde{K}(k-Q)+\widetilde{K}_{o s}(k-Q)\right) a_{-}(t) e^{i(k-Q) x} \\
= & \lambda(k+Q) a_{+}(t) e^{i(k+Q) x}+\lambda(k-Q) a_{-}(t) e^{i(k-Q) x}
\end{aligned}
$$

$$
\begin{aligned}
\hat{L}_{k}^{2}\left[\psi_{k}^{Q}\right]= & -A_{k}^{2}\left(2+\frac{4 \widetilde{K}_{o s}(0)}{\sigma_{z}^{2}}-\frac{4 \widetilde{K}_{o s}(k)}{\sigma_{z}^{2}}\right) a_{+}(t) e^{i(k+Q) x} \\
& -A_{k}^{2}\left(2+\frac{4 \widetilde{K}_{o s}(0)}{\sigma_{z}^{2}}-\frac{4 \widetilde{K}_{o s}(k)}{\sigma_{z}^{2}}\right) a_{-}(t) e^{i(k-Q) x}
\end{aligned}
$$

$$
\begin{aligned}
\hat{L}_{k}^{3}\left[\psi_{k}^{Q}\right]= & -A_{k}^{2}\left(1+\frac{\widetilde{K}_{o s}(0)}{\sigma_{z}^{2}}-\frac{2 \widetilde{K}_{o s}(k)}{\sigma_{z}^{2}}+\frac{\widetilde{K}_{o s}(2 k)}{\sigma_{z}^{2}}\right) a_{+}(t) e^{i(k-Q) x} \\
& -A_{k}^{2}\left(1+\frac{\widetilde{K}_{o s}(0)}{\sigma_{z}^{2}}-\frac{2 \widetilde{K}_{o s}(k)}{\sigma_{z}^{2}}+\frac{\widetilde{K}_{o s}(2 k)}{\sigma_{z}^{2}}\right) a_{-}(t) e^{i(k+Q) x}
\end{aligned}
$$

$$
\begin{aligned}
\hat{L}_{k}^{4}\left[\psi_{k}^{Q}\right]= & -A_{k}^{2} \int_{\mathbb{R}} \frac{K_{o s}(x-y)}{\sigma_{z}^{2}}\left(-4+2 e^{i k(x-y)}+2 e^{-i k(x-y)}\right)\left(a_{+}(t) e^{i(k+Q) y}+a_{-}(t) e^{i(k-Q) y}\right) d y \\
= & -A_{k}^{2} \int_{\mathbb{R}} \frac{K_{o s}(x-y)}{\sigma_{z}^{2}}\left(-4\left(a_{+}(t) e^{i(k+Q) y}+a_{-}(t) e^{i(k-Q) y}\right)\right) d y \\
& -A_{k}^{2} \int_{\mathbb{R}} \frac{K_{o s}(x-y)}{\sigma_{z}^{2}}\left(+2 e^{i k x}\left(a_{+}(t) e^{i(Q) y}+a_{-}(t) e^{i(-Q) y}\right)\right) d y \\
& -A_{k}^{2} \int_{\mathbb{R}} \frac{K_{o s}(x-y)}{\sigma_{z}^{2}}\left(2 e^{-i k x}\left(a_{+}(t) e^{i(2 k+Q) y}+a_{-}(t) e^{i(2 k-Q) y}\right)\right) d y \\
= & -2 A_{k}^{2}\left(-2 \frac{\widetilde{K}_{o s}(k+Q)}{\sigma_{z}^{2}}+\frac{\widetilde{K}_{o s}(Q)}{\sigma_{z}^{2}}+\frac{\widetilde{K}_{o s}(2 k+Q)}{\sigma_{z}^{2}}\right) a_{+}(t) e^{i(k+Q) x} \\
& -2 A_{k}^{2}\left(-2 \frac{\widetilde{K}_{o s}(k-Q)}{\sigma_{z}^{2}}+\frac{\widetilde{K}_{o s}(Q)}{\sigma_{z}^{2}}+\frac{\widetilde{K}_{o s}(2 k-Q)}{\sigma_{z}^{2}}\right) a_{-}(t) e^{i(k-Q) x}
\end{aligned}
$$




$$
\begin{aligned}
\hat{L}_{k}^{5}\left[\psi_{k}^{Q}\right]= & -A_{k}^{2} \int_{\mathbb{R}} \frac{K_{o s}(x-y)}{\sigma_{z}^{2}}\left(2 e^{i k(x+y)}+e^{2 i k y}-e^{2 i k x}\right)\left(a_{+}(t) e^{i(-k-Q) y}+a_{-}(t) e^{i(-k+Q) y}\right) d y \\
= & -A_{k}^{2} \int_{\mathbb{R}} \frac{K_{o s}(x-y)}{\sigma_{z}^{2}}\left(2 e^{i k(x)}\left(a_{+}(t) e^{i(-Q) y}+a_{-}(t) e^{i(Q) y}\right)\right) d y \\
& -A_{k}^{2} \int_{\mathbb{R}} \frac{K_{o s}(x-y)}{\sigma_{z}^{2}}\left(\left(a_{+}(t) e^{i(k-Q) y}+a_{-}(t) e^{i(k+Q) y}\right)\right) d y \\
& -A_{k}^{2} \int_{\mathbb{R}} \frac{K_{o s}(x-y)}{\sigma_{z}^{2}}\left(-e^{2 i k x}\left(a_{+}(t) e^{i(-k-Q) y}+a_{-}(t) e^{i(-k+Q) y}\right)\right) d y \\
= & -A_{k}^{2}\left(2 \frac{\widetilde{K}_{o s}(Q)}{\sigma_{z}^{2}}+\frac{\widetilde{K}_{o s}(k+Q)}{\sigma_{z}^{2}}-\frac{\widetilde{K}_{o s}(k-Q)}{\sigma_{z}^{2}}\right) a_{-}(t) e^{i(k+Q) x} \\
& -A_{k}^{2}\left(2 \frac{\widetilde{K}_{o s}(Q)}{\sigma_{z}^{2}}+\frac{\widetilde{K}_{o s}(k-Q)}{\sigma_{z}^{2}}-\frac{\widetilde{K}_{o s}(k+Q)}{\sigma_{z}^{2}}\right) a_{+}(t) e^{i(k-Q) x}
\end{aligned}
$$

To shorten the notation when bringing terms together, $\Gamma(k, Q), Y_{+}(k, Q)$ and $Y_{-}(k, Q)$ are defined as

$$
\begin{aligned}
\Gamma(k, Q) & =\widetilde{K}_{o s}(0)-2 \widetilde{K}_{o s}(k)+2 \widetilde{K}_{o s}(Q)+\widetilde{K}_{o s}(2 k)-\widetilde{K}_{o s}(k+Q)-\widetilde{K}_{o s}(k-Q) \\
\mathrm{Y}_{ \pm}(k, Q) & =2 \widetilde{K}_{o s}(0)-2 \widetilde{K}_{o s}(k)+\widetilde{K}_{o s}(Q)-2 \widetilde{K}_{o s}(k \pm Q)+\widetilde{K}_{o s}(2 k \pm Q)
\end{aligned}
$$

This way, the entries of the matrix are

$$
M_{2 \times 2}=\left(\begin{array}{cc}
\lambda(k+Q)-2 A_{k}^{2}\left(1+\frac{1}{\sigma_{z}^{2}} Y_{+}(k, Q)\right) & -A_{k}^{2}\left(1+\frac{1}{\sigma_{z}^{2}} \Gamma(k, Q)\right) \\
-A_{k}^{2}\left(1+\frac{1}{\sigma_{z}^{2}} \Gamma(k, Q)\right) & \lambda(k-Q)-2 A_{k}^{2}\left(1+\frac{1}{\sigma_{z}^{2}} Y_{-}(k, Q)\right)
\end{array}\right)
$$

The eigenvalues of $M_{2 \times 2}$ are

$$
\begin{aligned}
\mu_{1,2}(k, Q)= & \left(\frac{\lambda(k+Q)+\lambda(k-Q)}{2}\right)-2 A_{k}^{2}\left(1-\frac{1}{2 \sigma_{z}^{2}}\left(Y_{-}(k, Q)+Y_{+}(k, Q)\right)\right) \\
& \pm \sqrt{\left(\left(\frac{\lambda(k-Q)-\lambda(k+Q)}{2}\right)-\frac{A_{k}^{2}}{\sigma_{z}^{2}}\left(Y_{-}(k, Q)-Y_{+}(k, Q)\right)\right)^{2}+\left(A_{k}^{2}+\frac{A_{k}^{2}}{\sigma_{z}^{2}} \Gamma(k, Q)\right)^{2}}
\end{aligned}
$$

Equation 2.2.8 is an analytical expression for the stability of periodic stationary patterns. For a given combination of interaction parameters in the kernels $K$ and $K_{o s}, \mu_{1,2}(k, Q)$ gives the eigenvalues of all possible perturbations $Q$ on a pattern with wavenumber $k$. If $\mu$ is positive for any $Q$, then the stationary pattern $k$ is unstable. The eigenvalue with the positive root will be used since it gives a higher number. In the following section it will be shown that for a special regime of $K$ and $K_{o s}$, $\mu(k, Q)>0$ for at least one $Q$ for every $k$. Since all organized patterns are unstable in that case, but the non-selective state is also unstable, the result is a disorganized layout of orientation preferences. 


\section{Uniformly selective state}

The uniformly selective state is a special case of the periodic states, where $k=0$. The stability of this kind of patterns is calculated using equation 2.2.8 as $\mu(k=0, Q)$.

\subsection{The transition from order to disorder}

In the previous section the conditions for the invariant solution $z(x)=A e^{i k x}$ to be stationary were derived in a one dimensional cortex. The eigenvalues in equation 2.2.6 and in equation 2.2.8 define conditions for their stability. The expressions hold for any choice of interaction parameters in $K$ and $K_{o s}$. In this section the range of interaction parameters will be reduced and narrowed by including recent experimental findings in the rodent visual cortex. The reduced parameters will be inserted in the derived equations to calculate which patterns are stationary solutions and are stable under perturbations. An interaction regime will be shown where no stationary pattern is stable, demonstrating that spatially periodic arrangements of orientation preferences will not form.

\subsubsection{Simplifying interaction parameters}

The interaction kernels in equation 2.1.3 have 9 parameters $\left(E, \sigma, I, \varrho, \sigma_{z}, E_{o s}, \sigma_{o s}, I_{o s}, \varrho_{o s}\right)$, describing a large set of possible shapes. To describe a coherent rodent interaction kernel, the amount of parameters is reduced to three $(S, W, g)$, where some of the original parameters are fixed and others have been combined. To do so, as in the simulations in section 2.1.3, the following experimental findings are used:

1. In the rodent visual cortex only excitatory interactions are orientation selective, while inhibitory neurons connect with their neighbors independent of their tuning [115]. This means that $I_{o S}=$ 0 .

2. There are no long-range orientation selective connections in the rodent visual cortex, meaning that the selective and unselective excitatory interactions can be assumed to have the same range [123]. This makes $\sigma_{o s}=\sigma$.

Brought together this reduces the kernels in equation 2.1.3 to

$$
\begin{gathered}
K\left(x_{i}-x_{j}\right)=\frac{E}{\sqrt{2 \pi} \sigma} e^{-\frac{\left(x_{i}-x_{j}\right)^{2}}{2 \sigma^{2}}}-\frac{I}{\sqrt{2 \pi} \varrho} e^{-\frac{\left(x_{i}-x_{j}\right)^{2}}{2 \rho^{2}}} \\
K_{o S}\left(x_{i}-x_{j}\right)=\frac{E_{O S}}{\sqrt{2 \pi} \sigma} e^{-\frac{\left(x_{i}-x_{j}\right)^{2}}{2 \sigma^{2}}}
\end{gathered}
$$

Furthermore, since only the shape of the interaction is important and not the total magnitude, the amount of parameters is reduced by combining them:

- The strength of excitation and inhibition will be controlled with a single parameter $S \in[0,1]$, where $S$ is the strength of excitation and $(1-S)$ the strength of inhibition. For $S=0$ there is only inhibition and for $S=1$ only excitation. 
- Similarly, the range of the excitatory and inhibitory interactions is controlled with the parameter $W \in(0,1)$, where $W \sigma$ is the range of excitation and $(1-W) \sigma$ the range of inhibition. $\sigma$ defines a typical interaction distance between neurons in the cortex and can be set to 1 without loss of generality. For $W<\frac{1}{2}$ the interaction will have a Mexican hat shape, while with $W>\frac{1}{2}$ the Mexican hat will be inverted.

- The total amount of excitation is split in the specific and unspecific interactions regulated by the parameter $g \in[0,1] . g$ scales the selective interaction and $(1-g)$ the unselective.

The resulting interaction kernels are

$$
\begin{gathered}
K\left(x_{i}-x_{j}\right)=\frac{(1-g) S}{\sqrt{2 \pi} W \sigma} e^{-\frac{\left(x_{i}-x_{j}\right)^{2}}{2 W^{2} \sigma^{2}}}-\frac{1-S}{\sqrt{2 \pi}(1-W) \sigma} e^{-\frac{\left(x_{i}-x_{j}\right)^{2}}{2(1-W)^{2} \sigma^{2}}} \\
K_{o s}\left(x_{i}-x_{j}\right)=\frac{g S}{\sqrt{2 \pi} W \sigma} e^{-\frac{\left(x_{i}-x_{j}\right)^{2}}{2 W^{2} \sigma^{2}}}
\end{gathered}
$$

In figure 2.3.1a) a graphical representation of the simplified kernel is given. Notice that the selectivity of the interactions is regulated both by parameters $\sigma_{z}$ and $g$. For a given $S$ and $W$, the probability of excitatory interaction $P\left(\theta_{i}-\theta_{j}\right)$ is proportional to

$$
P\left(\theta_{i}-\theta_{j}\right) \propto(1-g)+g e^{-\frac{\left|z_{j}-z_{i}\right|^{2}}{\sigma_{z}^{2}}}
$$

Continuing with the assumption that the selectivity of the interactions is of the same order of the selectivity of the tuning, $\sigma_{z} \simeq \mathcal{O}(z)$, the probability can be rewritten as

$$
P\left(\theta_{i}-\theta_{j}\right) \propto(1-g)+g\left(1-\frac{\left|z_{i}-z_{j}\right|^{2}}{\sigma_{z}^{2}}\right)=1-\frac{g}{\sigma_{z}^{2}}\left|z_{i}-z_{j}\right|^{2}
$$

Using $\sigma_{z}^{2}=|A|^{2}$ and $z=A e^{2 i \theta}$ this simplifies to

$$
P\left(\theta_{i}-\theta_{j}\right) \propto 1-g\left|e^{2 i \theta_{i}}-e^{2 i \theta_{j}}\right|^{2}
$$

The interaction probability has a cosine shape. When there is no difference in orientation between both neurons $P(0) \propto 1$. The lowest probability is with orthogonal orientations, $P\left(\frac{\pi}{2}\right) \propto 1-4 g$. This sets an effective range for $g$ where the expansion $\sigma_{z} \simeq \mathcal{O}(z)$ holds, $g \in\left[0, \frac{1}{4}\right]$. Experimentally it was reported that $P\left(\frac{\pi}{2}\right) / P(0) \approx 0.5$ [115], for which $g=\frac{1}{8}$. Figure 2.3.1b) gives a graphical representation of the selectivity of excitation for increasing differences in orientation between the neurons.

Using those steps, the parameters describing the rodent interaction are $S \in[0,1], W \in(0,1)$ and $g \in\left[0, \frac{1}{4}\right]$. With no orientation selective inhibition the denominator in the expression of the selectivity in equation 2.2.2 is always positive, so the definition of $\tilde{r}$ as in equation 2.2.3 is used. 

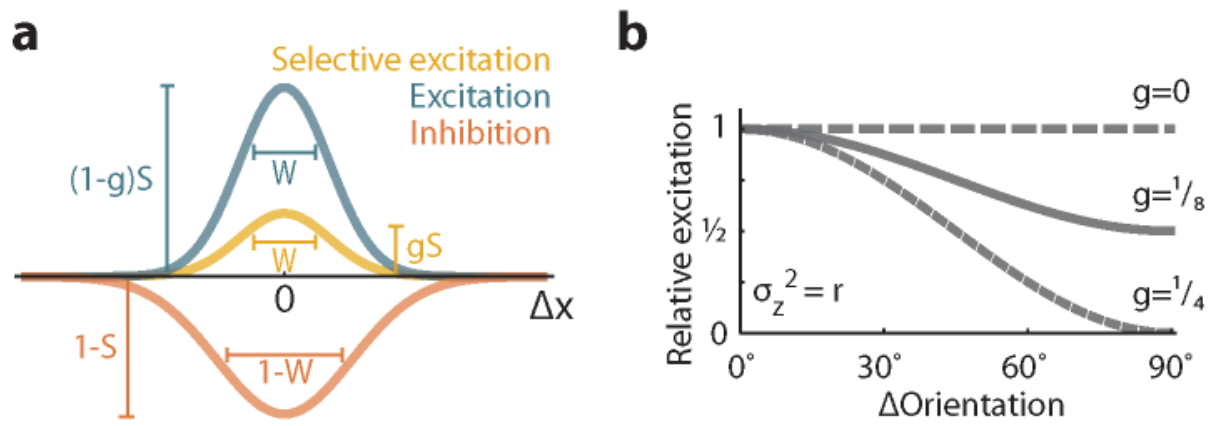

Figure 2.3.1: Graphical explanation of the simplified interaction parameters. a) The parameters for the unselective interaction (blue and red) are coupled with the parameters $S$ and $(1-S)$ for the strength and $W$ and $(1-W)$ for the range of excitation and inhibition respectively. There is only orientation selective excitation (yellow), which is linked to the unselective excitation with the parameter $g$ and $(1-g)$. b) The effect on the strength of excitation between neurons for increasing difference in orientation is depicted with different choices of the parameter $g$. For $g=0$ the excitatory interactions are independent of the orientation of the neurons. For $0<g \leq \frac{1}{4}$ the interaction is stronger for neurons where the orientation is more similar.

\subsubsection{Stationary solutions and their stability}

The simplified interaction kernels in equations 2.3.1 and 2.3.2 have the advantage of being described with only three bounded parameters. Therefore, for all possible combinations of $S, W$ and $g$ equation 2.2.2 can be used to find the patterns that are stationary and equations 2.2.6 and 2.2.8 to calculate their stability comprehensively screening all exact solutions for all parameters combinations.

\section{Unselective state}

As stated before, $A=0$ is always a stationary solution independent of the combination of parameters. Using the definition of $\tilde{r}$ as in equation 2.2.3, $\lambda_{\max }(k)=r>0$, so the unselective state is not stable and the selectivity $A$ will increase with time.

\section{Perfectly periodic and uniformly selective state}

The condition for $z=A_{k} e^{i k x}$ to be stationary is $A_{k}>0$ in equation 2.2.2. Since there is no orientation selective inhibition, this condition is fulfilled when $\lambda(k)>0$ in equation 2.2.6. With the reduced interaction kernels the expression for $\lambda$ is independent of $g$

$$
\lambda(k)=\tilde{r}+S e^{-\frac{1}{2} k^{2} W^{2}}-(1-S) e^{-\frac{1}{2} k^{2}(1-W)^{2}}
$$

The range of patterns $k$ that make $\lambda(k)>0$ depends on the choice of $r$ in equation 2.2.3. The focus is on the maximum of $\lambda$ since it is always positive even with very small $r . \lambda(k)$ has two extreme points

$$
k_{I}=0 \text { and } k_{I I}=\sqrt{\frac{\log \left(\frac{(1-S)(1-W)^{2}}{S W^{2}}\right)}{\frac{1}{2}-W}}
$$

They exchange characteristics depending on the choice of $S$ and $W$ : 
a

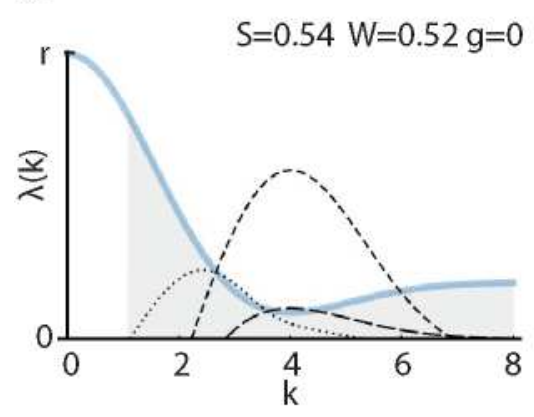

b

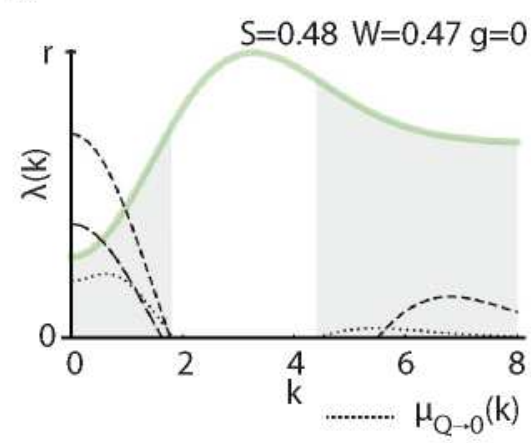

C

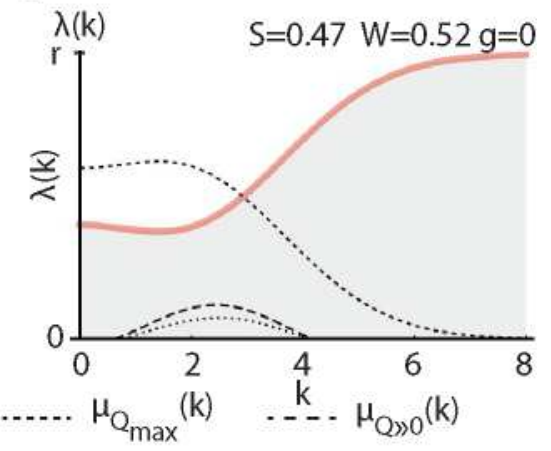

Figure 2.3.2: Stability of stationary patterns. a) - c) The growth rate of the stationary pattern $\lambda(k)$ is plotted for a combination of interactions parameters belonging to a) type I, b) type II and c) type III. On top three examples of the growth rate of a perturbation over the stationary pattern $\mu(k, Q)$ is plotted, with $Q \rightarrow 0$ (dotted line), with the maximum of $\mu$ for a given $k$ : $Q_{\max }$ (short dashed line), and with $Q \rightarrow \infty$ (long dashed line). The patterns with wavenumber $k$ where the growth rate of the instability is positive for any $Q$ are unstable and are marked with gray.

- Type I interaction: For $\frac{(1-S)(1-W)^{2}}{S W^{2}} \leq 1$ and $S \geq \frac{1}{2}, k_{I}$ is the maximum and $\tilde{r}=r-(2 S-1)$. With these parameters the uniformly selective state $k=0$ is stationary.

- Type II interaction: For $\frac{(1-S)(1-W)^{2}}{S W^{2}}>1$ and $W<\frac{1}{2}, k_{I I}$ is maximum and $\tilde{r}=r-\left(S\left(\frac{(1-S)(1-W)^{2}}{S W^{2}}\right)^{\frac{W^{2}}{-1+2 W}}-(1-S)\left(\frac{(1-S)(1-W)^{2}}{S W^{2}}\right)^{\frac{(1-W)^{2}}{-1+2 W}}\right)$. Choosing $S$ and $W$ in this range makes the perfectly periodic state stationary.

- Type III interaction: For $S=0$ or for $S \leq \frac{1}{2}$ and $W \geq \frac{1}{2}$, the single maximum at finite wave number is lost and an infinite number of modes satisfy $\lambda(k)>0$. In this case, even with small $r$, there is a continuum of periodic patterns with arbitrary high wave numbers that are stationary.

In every interaction type the stability of the stationary patterns will be analyzed. An example of this procedure for a limited number of perturbations is shown in figure 2.3.2a) to c). In every figure the growth rate of an ordered solution $\lambda(k)$ in equation 2.3.3 is plotted for different interaction parameters belonging to interaction type I, II and II respectively. The bifurcation parameter is $r=0.1$. Plotted on top of each curve are three examples of the growth rate of the perturbation $\mu(k)$ from equation 2.2.8, with $Q \rightarrow 0$ (dotted line), with the maximum of $\mu$ for a given $k$ : $Q_{\max }$ (short dashed line), and with $Q \rightarrow \infty$ (long dashed line). In all examples, whenever $\mu(k)$ is positive for any given $Q$ the pattern $z=A e^{i k x}$ is unstable and is marked in the figures with gray. Using only this limited number of instabilities $Q$ in the example, with type I interaction in a) the stable pattern with fastest growth is $k=0$, with type II interaction in $\mathrm{b}$ ) the stable pattern has a finite wavelength $k>0$, and with interaction type III in c) all ordered patterns are unstable. Having this in mind, next the different interaction types will be treated separately showing that the results of this example are general for the whole interaction parameters in each type. As stated, the focus is on the maximum of $\lambda(k)$ in equation 2.3.4, since it is always positive even with very small $r$. 
Type I interaction Inserting $k_{I}=0$ in equation 2.2.8 leads to $\Gamma(0, Q)=\mathrm{Y}_{ \pm}(0, Q)=0$ and $\left|A_{0}\right|^{2}=$ $\lambda(0)$. The eigenvalue of the perturbation can be written as

$$
\mu(k=0, Q)=\lambda(Q)-\lambda(0)
$$

Since in interaction type I $\lambda(0)$ is maximum, $\mu \leq 0$ for all $Q$, making the continuous pattern stable in this regime. In this interaction regime the emerging patterns will always be a uniform orientation preference.

Type II interaction For the interaction regime type II where there is a single maximum at $k_{I I}=$ $\sqrt{\frac{\log \left(\frac{(1-S)(1-W)^{2}}{S W^{2}}\right)}{\frac{1}{2}-W}}$ the stability of the pattern $z(x)=A^{i k_{I I} x}$ can not be proved analytically with only one equation as in type I. Here the focus is on three different perturbation types for the unselective interaction case $(g=0), Q \rightarrow 0, Q_{\max }$ and $Q \rightarrow \infty$. In all those cases the pattern with wavenumber $k_{I I}$ will be shown to be stable. In the next section the stability of the map will be investigated numerically for more $Q$ and $g \neq 0$, showing that the results also holds for those cases.

Long wavelength perturbation $Q \rightarrow 0$ : Since many derivatives are involved in the next steps, the following notation will be used

$$
\begin{aligned}
& \psi(k)=S e^{-\frac{1}{2} k^{2} W^{2}} \\
& \xi(k)=(1-S) e^{-\frac{1}{2} k^{2}(1-W)^{2}}
\end{aligned}
$$

Equation 2.2.8 will be expanded in Taylor series around zero, such that $Q \simeq 0$. Only even terms of $Q$ will be non-zero because of the symmetry of the system. Up to second order the terms are

$$
\begin{aligned}
\mu_{Q \rightarrow 0}(k, Q)= & \frac{k^{2}}{2}\left(\frac{\left(W^{2} \psi(k)-(1-W)^{2} \xi(k)\right)^{2}}{A^{2}}+\left(W^{4} \psi(k)-(1-W)^{4} \xi(k)\right)\right) Q^{2} \\
& -\frac{1}{2}\left(W^{2} \psi(k)-(1-W)^{2} \xi(k)\right) Q^{2}+\mathcal{O}\left(Q^{3}\right)
\end{aligned}
$$

Inserting the maximum $k_{I I}$ leads to

$$
\mu_{Q \rightarrow 0}\left(k_{I I}, Q\right)=-S W^{2}\left(\frac{(1-S)(1-W)^{2}}{S W^{2}}\right)^{\frac{W^{2}}{-1+2 W}} \log \left(\frac{(1-S)(1-W)^{2}}{S W^{2}}\right) Q^{2}+\mathcal{O}\left(Q^{3}\right)
$$

This is smaller than zero for $\frac{(1-S)(1-W)^{2}}{S W^{2}}>1$, which is part of the type II interaction. The pattern $z(x)=A\left(k_{I I}\right) e^{i k_{I I} x}$ is stable under this kind of perturbations. 
Finite wavelength perturbation $Q_{\max }$ : For the finite wavelength perturbation the extrema of the eigenvalue $\mu$ with respect of $Q$ will be found and their effect on the stationary pattern will be calculated

$$
\begin{aligned}
\frac{\partial \mu(k, Q)}{\partial Q}= & \frac{(k+Q)}{2}\left(W^{2} \psi(k+Q)-(1-W)^{2} \xi(k+Q)\right)(-1+\gamma(k, Q)) \\
& +\frac{(k-Q)}{2}\left(W^{2} \psi(k-Q)-(1-W)^{2} \xi(k-Q)\right)(1+\gamma(k, Q))
\end{aligned}
$$

with

$$
\gamma(k, Q)=\frac{\psi(k-Q)-\xi(k-Q)-\psi(k+Q)+\xi(k+Q)}{\sqrt{(\psi(k-Q)-\xi(k-Q)-\psi(k+Q)+\xi(k+Q))^{2}+4 A^{4}}}
$$

To set equation 2.3.5 to zero, first notice that $\gamma= \pm 1$ only when $A=0$, which is not a stationary pattern. The derivative consists of two parts, where only the sign of $Q$ is changed. $\mu$ is even, so $\frac{\partial \mu(k, Q)}{\partial Q}$ is odd and the zeros will be symmetric. The zeros of the first term will be modified by the zeros of the second term and the other way around. Since there is not an analytical expression that sets both parts to zero simultaneously, the location of the maximum is approximated using only the term that gives the positive zeros. Those are

$$
Q_{1}=k \quad Q_{2,3}=k \pm k_{I I}
$$

- Using the first extreme point $Q_{1}=k$ leads to

$$
\mu\left(k=k_{I I}, Q=k_{I I}\right)=-r+\frac{r_{I}-r_{I I}}{2}+\sqrt{r^{2}+\left(\frac{r_{I}-r_{I I}}{2}\right)^{2}}
$$

with $r_{I}=2 S-1$ and $r_{I I}=S\left(\frac{(1-S)(1-W)^{2}}{S W^{2}}\right)^{\frac{W^{2}}{-1+2 W}}-(1-S)\left(\frac{(1-S)(1-W)^{2}}{S W^{2}}\right)^{\frac{(1-W)^{2}}{-1+2 W}}$. The growth rate is negative for $W<\frac{1}{2}$ since $r_{I}<r_{I I}$ in this case. With $W<\frac{1}{2}, \frac{(1-S)(1-W)^{2}}{S W^{2}}>1$ to have a pattern with real wave number $k$ in equation 2.3.4. Since in interaction regime II this holds, the pattern $z(x)=A\left(k_{I I}\right) e^{i k_{I I} x}$ is stable under this perturbation.

- For the second extreme point, $Q_{2}=k-k_{I I}=0$ and there is no perturbation.

- For the third extreme point $Q_{3}=k+k_{I I}=2 k_{I I}$

$$
\mu\left(k=k_{I I}, Q=2 k_{I I}\right)=-r+\frac{-r_{I I}+\psi\left(3 k_{I I}\right)-\xi\left(3 k_{I I}\right)}{2}+\sqrt{r^{2}+\left(\frac{-r_{I I}+\psi\left(3 k_{I I}\right)-\xi\left(3 k_{I I}\right)}{2}\right)^{2}}
$$

This expression is negative for $W<\frac{1}{2}$, so $\frac{(1-S)(1-W)^{2}}{S W^{2}}>1$, making the wave number $k_{I I}$ stable with this interaction type II. 
Short wavelength perturbation $Q \rightarrow \infty$ : Setting $Q \rightarrow \infty$ for $\lambda(k)>0$ leads to

$$
\mu_{Q \rightarrow \infty}(k)=(1-S) e^{-\frac{k^{2}(1-W)^{2}}{2}}-S e^{-\frac{k^{2} W^{2}}{2}} .
$$

Inserting $k_{I I}$ this gives the normalization constant,

$r_{I I}=S\left(\frac{(1-S)(1-W)^{2}}{S W^{2}}\right)^{\frac{W^{2}}{-1+2 W}}-(1-S)\left(\frac{(1-S)(1-W)^{2}}{S W^{2}}\right)^{\frac{(1-W)^{2}}{-1+2 W}}$. If $W<\frac{1}{2}$ then $r_{I I}<0$, therefore the pattern is stable under this kind of perturbations.

Type III interaction For the interaction regime where there is no single maximum a short wavelength perturbation is used, $Q \rightarrow \infty$. For $\lambda(k)>0$, the growth rate of the perturbation can be written as

$$
\mu_{Q \rightarrow \infty}(k)=(1-S) e^{-\frac{k^{2}(1-W)^{2} \sigma^{2}}{2}}-S e^{-\frac{k^{2} W^{2} \sigma^{2}}{2}}-2 g\left(S e^{-\frac{k^{2} W^{2} \sigma^{2}}{2}}-S e^{-2 k^{2} W^{2} \sigma^{2}}\right)
$$

The eigenvalue of the instability decrease exponentially with $k$. For $S=0$ the growth rate is positive for all $k$. For $S>0$ the condition for instability $\mu_{Q \rightarrow \infty}(k)>0$ can be rewritten as

$$
\log \left(\frac{1-S}{S}\right)>k^{2}\left(\frac{1}{2}-W\right)+\log \left(1+2 g\left(1-e^{-\frac{3}{2} k^{2} W^{2}}\right)\right)
$$

With $g=0$, i.e. with no orientation specific connections, for $S<\frac{1}{2}$ the left side hand is positive (zero for $S=\frac{1}{2}$ ) and for $W>\frac{1}{2}$ the right hand side negative (zero for $W=\frac{1}{2}$ ), satisfying the condition of instability for all $k$ in this interaction type. With $g>0$, the second part of the right hand side is zero at $k=0$ and otherwise positive with maximum $\log (1.5)$ for large $k$ and $g=\frac{1}{4}$. This only modifies the stability when $S \simeq \frac{1}{2}$ and $W \simeq \frac{1}{2}$ for small $k$. In general, in this interaction regime all patterns, although being stationary solutions, are not stable under perturbations. Even with orientation selective interactions no ordered arrangement of neurons is possible in this regime, so the continuum limit $N \rightarrow \infty$ can not be used and the neurons have to be defined individually as $z_{i}=A_{i} e^{i 2 \theta_{i}}$.

\subsubsection{Numerical confirmation of results}

\section{Busse balloons and phase diagram}

Busse balloons Busse balloons, common in pattern formation physics, are a graphical way of representing stability regions in the parameter space for different wavenumbers of the pattern. Since the stability calculations are kept in the background and only the results are shown, they help to have a clear idea of phase transitions that occurs in the model as parameters vary.

Using the defined interaction kernels in equation 2.3.1 and equation 2.3.2, one can test for different combinations of the parameters $r, S, W$ and $g$ which organized patterns are stationary using $\lambda(k)$ in equation 2.2.6, and which of those are stable under perturbations using $\mu(k, Q)$ in equation 2.2.8. Fixing the parameters of the interaction, the wave number $k$ was increased stepwise from $k_{\min }$ to $k_{\max }$ in steps $\Delta k$. If a value $k_{i}$ was found such that $\lambda\left(k_{i}\right)>0$, then $Q$ was varied from $Q_{\text {min }}$ to $Q_{\text {max }}$ in steps 
a map unstable map stable

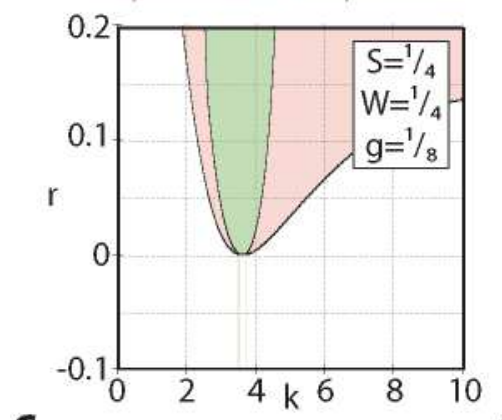

C

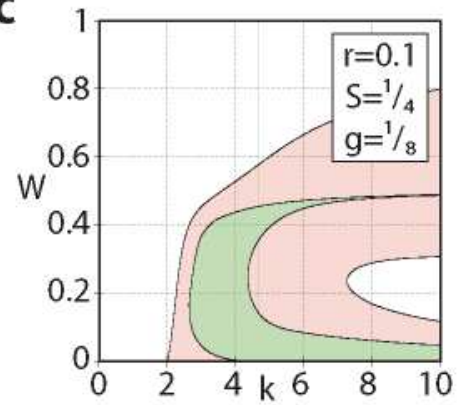

b

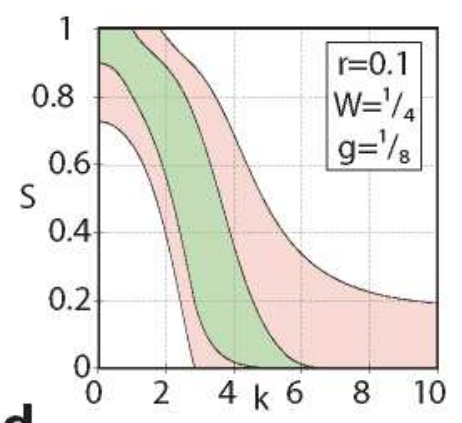

d

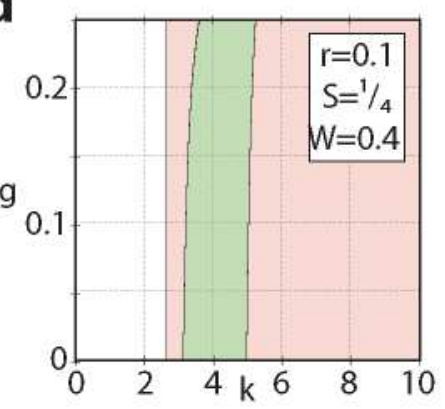

e

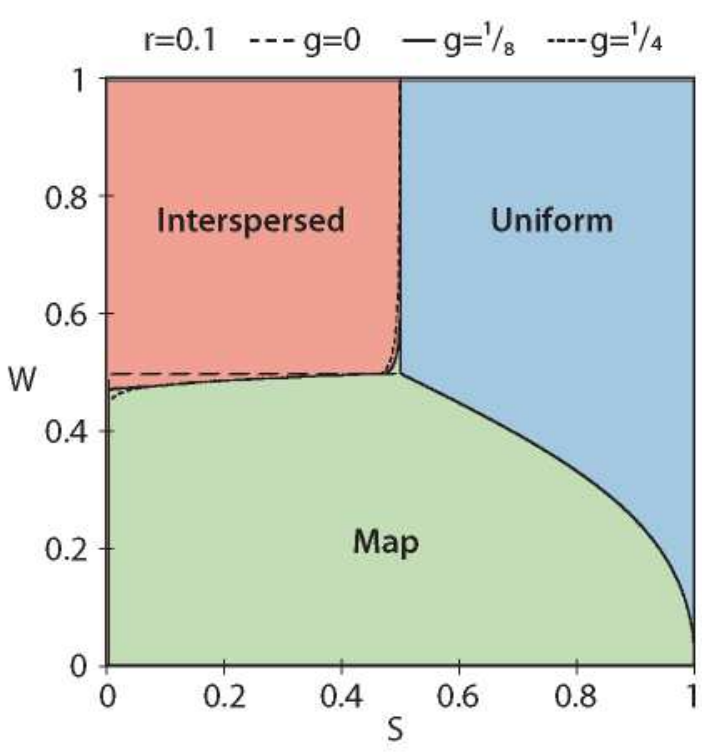

Figure 2.3.3: Numerical confirmation of the analytical results using the stability equations. a) - d) Busse balloons showing the stable regions in the parameter space for different patterns with wavenumber $k$. Unstable patterns are marked in red and stable in green. a) Role of the bifurcation parameter $r$ in the emergence of selectivity from the homogeneous state. b) Role of the strength of the interactions in the stability patterns. With high $S$ the continuous state $k=0$ is stable, with lowered $S$ the stable pattern is periodic with $k>0$ and with $S=0$ all patterns are unstable. c) Role of the range of the interaction in the stability of the patterns. When the Mexican hat with low $W$ is inverted after $W=\frac{1}{2}$ all patterns are unstable. d) Role of the selectivity of the interactions. With increasing selectivity the stable region shifts towards higher wavenumber. e) Phase diagram of the solutions of the model. The colors are set using $g=\frac{1}{8}$ and overlaid are the phase transitions for $g=0$ with dashed lines and for $g=\frac{1}{4}$ with dotted lines. The analytical results are confirmed.

$\Delta Q$ and the expression $\mu\left(k_{i}, Q\right)$ was tested for its sign. If $\mu\left(k_{i}, Q_{k}\right)>0$ for a $Q_{k}$, then the variation in $Q$ was interrupted, the wave number $k_{i}$ was determined as unstable, and the procedure started again with $k=k_{i}+\Delta k$. To accelerate the procedure the key $Q$ found analytically is used and $\mu\left(k_{i}, Q\right)$ with $Q=\left\{10^{-5}, k-k_{\text {max }}, k, k+k_{\text {max }}, 10^{5}\right\}$ is explicitly tested, where $k_{\text {max }}$ is the maximum growing mode of $\lambda$ in the tested interaction type. The set of stable wave numbers $k_{s}$ for a specific combination of $S$, $W$ and $g$ was thus defined as

$$
k_{s}=\left\{k \epsilon\left[k_{\text {min }}, k_{\text {max }}\right]: \lambda(k)>0 \cap \mu(k, Q) \leq 0 \forall Q \epsilon\left[Q_{\text {min }}, Q_{\text {max }}\right]\right\}
$$

The equations were tested with $k_{\min }=0, k_{\max }=10, Q_{\min }=0, Q_{\max }=30$ and $\Delta k=\Delta Q=0.01$. Figure 2.3.3a) to d) shows the results of this procedure in Busse balloons for different combination of parameters: a) $\left.\left.S=\frac{1}{4}, W=\frac{1}{4}, g=\frac{1}{8}, \mathrm{~b}\right) r=0.1, W=\frac{1}{4}, g=\frac{1}{8}, \mathrm{c}\right) r=0.1, S=\frac{1}{4}, g=\frac{1}{8}$ and d) $r=0.1, S=\frac{1}{4}, W=\frac{2}{5}$. The patterns that are a stationary solution to the equation are labeled with red and green. Only the patterns labeled with green are stable under all tested perturbations $Q$.

In a) the role of the bifurcation parameter $r$ is shown. Only when $r>0$ symmetry is broken and 
organized patterns become solutions to the equation, but not all of those patterns are stable.

In $b$ ) the role of the strength of the interaction is illustrated. At the top of the figure excitation is strong, with interaction type I. In this case the stable region surrounds the continuous solution $k=0$, where all neurons are tuned to the same orientation. With a decrease of $S$, the interaction switches to type II, and the stable region is a periodic pattern with positive wavenumber $k>0$. With $S=0$ there is only inhibition and the interaction is type III. The stability region shrinks and then fully disappears as the parameter approaches this value. In this case all ordered solutions are unstable.

In c) the transition from order to disorder is further clarified. At the bottom part of the figure the interaction has a Mexican hat shape, making it a interaction type II. As the border $W=\frac{1}{2}$ is crossed, local inhibition gets stronger and the Mexican hat is inverted, making it a interaction type III. As in b), it can be seen that as this value is approached the stable regions shrinks and disappears. In the upper part of the figure organized patterns exist that are stationary solutions to the equation, marked in red, but all of them are unstable.

In d) the role of the orientation selective excitatory interactions is depicted. The stable region is only slightly modified even with high $g$. The selectivity of the interactions only plays a significant role close to the phase transitions for patterns with a high wavenumber $k \gg 0$ or with low wavenumber $k \simeq 0$.

Taken together the Busse balloons show that the results of the analytical calculation of the stability of the stationary patterns are solid. When the interaction is of type III all organized layouts of orientation preference that are stationary are not stable under perturbations.

Phase diagram The phase diagram summarizes the Busse balloons by summarizing the results for all patterns $k$ with a given combination of parameters (i.e moving horizontally in the diagram) into a single label. In principle there are 4 possible outcomes:

- Only solutions with low $k$ approximately 0 are stable (Uniform phase).

- The uniform pattern is unstable but at least one periodic pattern with $k>0$ is stable (Map phase).

- Uniform and periodic patterns are both stable (Coexistence phase).

- Both uniform and periodic patterns are unstable (Putative Interspersed phase).

As we saw above a coexistence phase is excluded in the model. For the phase diagram $S, W$ and $g$, the mode $k_{i}$ in $k_{s}$ with the maximum growth rate $\lambda\left(k_{i}\right)$ is used. If $k_{i}=0$, the point in phase space was labeled as uniform phase. If $k \neq 0$ the point was labeled as map phase. If $k_{s}$ was an empty set, the point was marked as interspersed phase. The limits used are $k_{\min }=0, k_{\max }=15, Q_{\min }=0$, $Q_{\max }=30$ and $\Delta k=\Delta Q=0.01$. $S$ was varied from 0 to 1 and $W$ from 0.01 to 0.99 in steps of 0.01 . Three different $g$ were used, $g=0, g=\frac{1}{8}$ and $g=\frac{1}{4}$.

Figure 2.3.3e) shows the resulting phase diagram, where the interspersed phase is red, the map phase is green and the uniform phase is blue. The phase coloring is done with $g=\frac{1}{8}$. The phase transition for $g=0$ is overlaid with dashed lines and for $g=\frac{1}{4}$ with dotted lines. For $g=0$ the phase transition corresponds to the transition between the interaction types, as was shown analytically. 
a

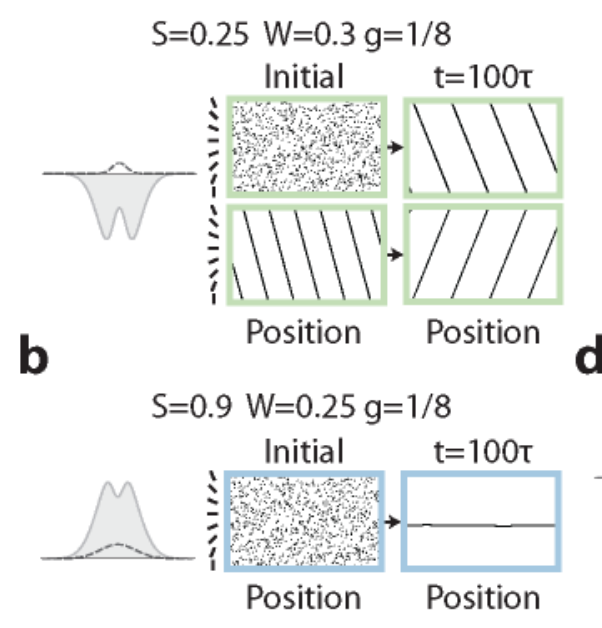

C
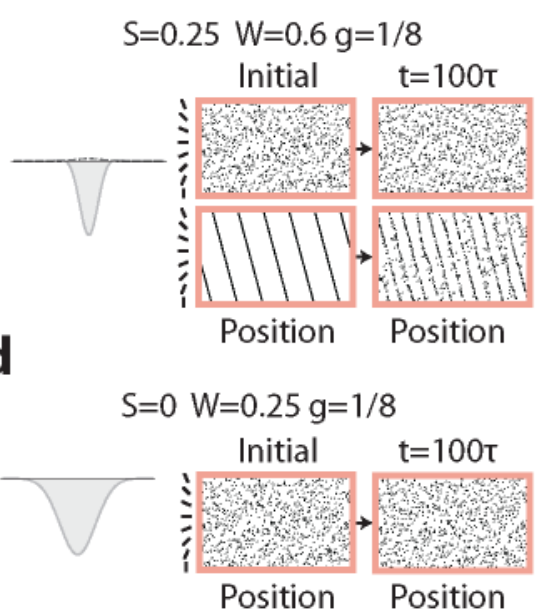

e

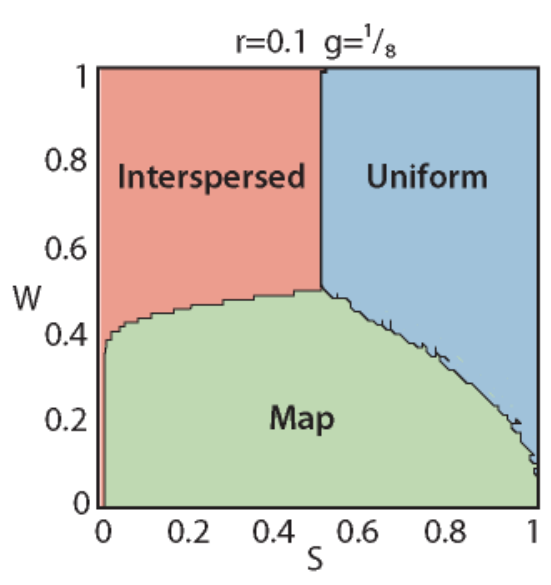

Figure 2.3.4: Numerical confirmation of the analytical results simulating a one dimensional cortex. a) to d) For given parameters the shape of the interaction is plotted left from the simulations. Unselective interactions are filled with gray and selective interactions are dashed. The layout of the one dimensional cortex is plotted with the position of the neuron in the $\mathrm{x}$-axis and the corresponding preferred orientation in the $\mathrm{y}$-axis. The initial condition is either a random arrangement of orientation preferences with low selectivity or a periodic pattern that is a stationary solution to the dynamics. In all simulations $r=0.1$. e) Reproduction of the phase diagram using the simulations. With the given parameters a random initial condition is used and the system develops for $t=500 \tau$. At the end of each simulation the wavenumber of the pattern is tested and the corresponding phase is assigned.

With increasing $g$ the general shape of the phases remains constant and the the only changes are observable close to the map - interspersed phase transition:

- When $S \simeq \frac{1}{2}$ and $W \simeq \frac{1}{2}$ the interaction is mostly a single Gaussian that flips from positive to negative as the value $W=\frac{1}{2}$ is passed from below. With increasing selectivity of the excitatory interaction $g$, part of this vanishing non-selective excitation is maintained, extending the map phase into the interspersed phase.

- When $S<\frac{1}{2}$ and $W \simeq \frac{1}{2}$ the fastest growing mode has a high wavenumber $k \gg 0$. At some point, this mode goes to infinity and all patterns become unstable. As can be seen from the Busse balloon of $g$ in $d$ ), by increasing $g$ neurons with a similar orientation get closer together, shifting the stability regime towards higher $k$. Therefore, the transition to the interspersed phase where instability kicks in starts earlier with increasing $g$, extending that phase into the map phase.

The phase diagram also confirms the analytical results. It is based on the first steps of the analysis of the orientation dynamics. The next test will directly start from the definition of the tuning dynamics in equation 2.1.4 and will use it to simulate the emergence of selectivity in a set of model neurons. 


\section{Simulation for a one dimensional grid of neurons}

In a second numerical approach the dynamical equation 2.1.4 was solved in a grid of $N=512$ equally spaced neurons in a box of size $L=2 \pi \sigma$ with periodic boundary conditions. The tuning of each neuron was described by a complex number, $z_{i}=A_{i} e^{i 2 \theta_{i}}$. The size of the box was set to prevent the interaction of neurons with their copy in the next box. $N$ was controlled to be high enough to have at least 12 neurons interacting within the shortest interaction width $\min (W,(1-W)) \sigma$. The discretization restricts the possible wave numbers to $-\frac{N}{2}<k<\frac{N}{2}+1$ with $k \in \mathbb{Z}$. The characteristic time constant of the simulations $\tau$ was defined as the reciprocal of the fastest growth rate from the homogeneous state $\lambda\left(k_{\max }\right)=r$, where $r=0.1$ was used. All $z_{i}$ were updated simultaneously in each discrete time step using a 4th-5th order Runge-Kutta-Fehlberg method. The error was controlled to be maintained below $0.01 r$. Periodic conditions were imposed by solving the convolutions of the grid of neurons with the kernels $K$ and $K_{o s}$ in Fourier space. The equation was solved without additive noise.

In figure 2.3.4a) to d) the stability of different initial conditions for given interaction parameters is assessed. In each figure the shape of the interaction kernels is schematized. The one dimensional system is plotted with the position of the neuron in the $x$-axis and the orientation in the y-axis. The initial conditions were either randomly distributed unselective tuning $z_{i}=\frac{1}{100} \sqrt{r \xi_{1}} e^{i 2 \xi_{2}}$, where $\xi_{1} \in \mathcal{U}[0,1]$ and $\xi_{2} \in \mathcal{U}[0, \pi]$ are uniformly distributed random numbers; or a noisy pattern with wave number $k$, $z_{i}=A(k) e^{i k x_{i}}+\frac{\xi_{3}}{100} e^{i 2 \pi \xi_{4}}$, where $\xi_{3} \in \mathcal{N}[0,1]$ and $\xi_{4} \in \mathcal{U}[0, \pi]$ are Gaussian and uniformly distributed random numbers respectively. The stability of the initial patterns with the used interaction parameters matches the one predicted by the theory in figure 2.3.3b) and c). In figure 2.3.4a) unselective random initial conditions lead to an ordered pattern of wavenumber $k=4$, which is part of the stable region in the corresponding Busse balloon. In comparison, $k=6$ is a stationary solution with those parameters, but is not stable and decays into a pattern with $k=4$. In 2.3.4b) parameters are chosen to have a interaction type I. Therefore random initial conditions lead to a pattern where all neurons share the same preferred orientation. In 2.3.4c), in the interspersed phase, the selectivity is increased from the random initial conditions (not shown), but no ordered pattern emerges. If the system is started from a pattern with $k=6$, which is a stationary solution according to the analytic treatment, the pattern decays into a disordered layout. Figure c) is the one dimensional parallel to the simulations in figure 2.1.1d) and e), where orientation selectivity increases but ordered layouts decay. As with the two dimensional examples, a small addition of noise or longer simulations reduces the remaining organization from the ordered initial condition. Finally, figure 2.3.4d) shows that inhibition alone can already produce interspersed patterns, as can be seen in the Busse balloon in figure 2.3.3e).

In figure 2.3.4e) the phase diagram is reproduced with simulations. For this neurons with randomly distributed unselective tuning were used as initial condition as in a) to d). The development was simulated for $t=500 \tau$ and the wave number of the final pattern was detected by finding the maximum of the power spectrum $k_{\max }$. To label the pattern as ordered, the maximum should have at least twice the power of the second highest mode and $P\left(k_{\max }\right) \geq \frac{1}{\sqrt{N}} \sum_{k \neq k_{\max }} P(k)$. If $k_{\max }=0$ the point was marked in phase space as uniform. If $k_{\max } \neq 0$ the point was marked as map phase. If no wave number was found that matched the described conditions, the point was labeled as interspersed. $S$ was varied from 0 to 1 and $W$ from 0.01 to 0.99 , both in steps of 0.01 . The selectivity of the interaction was set to $g=\frac{1}{8}$. Using different values leaves the resulting phase diagram unchanged since very high values of $k$ are indistinguishable to interspersed patterns and are labeled as such. The results shown 
essentially recover the analytical phase diagram in figure 2.3.3e), maintaining the same general shape of the different phases and the transitions. The transition between the map and the uniform phase is not smooth because in some cases both $k=0$ and $k=1$ are stable and randomly one of them emerges. As mentioned the interspersed phase is larger compared to the analytical results since close to the map transition the stable ordered patterns have a high $k$ and are indistinguishable to interspersed layouts with the labeling method used.

Taken together, these results show the validity of the analytical conclusions. From the simulations one can learn that depending on the interaction parameters the solution changes from ordered to disordered, but only from the analytical calculations one can understand that the reason for this is the instability for all ordered solutions for strong short-range inhibition.

\subsection{Main conclusions from the order-parameter model}

The simulations of the dynamical equation in figure 2.1.1 show that the model can generate both types of layouts found in nature: pinwheel-rich quasi-periodic maps as in primates and carnivores, and disordered arrangements of orientation preference as in rodents and lagomorphs. In both cases the emerging layout is stable for very long simulations. The shape of the interaction between the neurons determines the type of layout that is generated.

From the original model proposed by Wolf is is known that long-range orientation-selective interactions are necessary to make the a quantitatively realistic map solution stable. Otherwise the map decays into a pattern with a lower amount of Fourier modes and pinwheel density deviating from experimental observations (see figure 1.3.1). The results of this analysis applies to all dynamical models that satisfy the imposed symmetries in equations 1.3.1 to 1.3.4. In any dynamical model for pattern formation that doesn't include this type of interactions pinwheel-rich maps might emerge initially, but they won't be stable and for longer simulations the pattern will decay to a structurally simpler solution. This is the case in the elastic net model, as was first found in [22] and analyzed in detail in [60].

From the analytical treatment and simulations of the generalized model it can be seen that strong short-range inhibition generates orientation selective neurons, which are arranged disordered in space. The interspersed pattern that emerges is mostly determined by the unselective interactions, where orientation selective interactions only play a significant role close to the ordered-disordered phase transition. From the analytical equations in the one dimensional cortex one learns that the reason for the generation of the disordered pattern is that, with strong local inhibition, all ordered periodic arrangements that are by symmetry solutions of the model are unstable, leaving only disordered arrangements as solution.

In this model it is shown that the same conditions lead to the generation of interspersed patterns of orientation selective neurons that are disordered in the cortical plane. In the next section the condition of strong intra-cortical inhibition will be implemented in a correlation based model for the emergence of orientation selectivity, developed by Miller [53]. The model is less abstract and has a direct biological interpretation of the dynamical mechanisms involved. In this model it is shown that if the conditions to generate interspersed patterns are satisfied, the result will be orientation selective neurons that are disordered in space. Strong inhibition was also applied in the elastic net model by Keil in his doctoral thesis [145] by removing the continuity term representing excitatory lateral con- 
nections and allowing only interactions through normalizing soft-competition, which is inhibitory by definition. The removal of excitation in this model also leads to the formation of interspersed patterns. The next part of the thesis will give a brief look at this model when quantifying the properties of the disordered solutions.

In three completely distinct models for orientation preference development with very dissimilar levels of abstraction the increment of local inhibition is found to generate spatially disordered arrangements of orientation selective neurons. Biologically there is evidence for the existence of strong intra-cortical inhibition in rodents, where genetical tools have made it possible to separately analyze the role of different types of neurons in cortical function. In rodents inhibitory neurons are densely connected to the neurons in their surroundings [120,148], cortical responses to visual stimuli are dominated by synaptic inhibition in awake state [136], the activation of a cortical neuron suppresses the activity of lateral vicinity [149], and the increase in excitation triggered by visual stimulation is followed by strong inhibitory conductance a few milliseconds later [150]. On the other hand, in species with orientation maps the new genetical tools are not available at present and knowledge about the excitation/inhibition ratio is limited. The available evidence is discussed in the final chapter and appears to be in line with the prediction of substantially stronger local excitation than found in rodents.

\subsection{Strong intracortical inhibition in a LGN correlation based model}

From the order-parameter model it was found that strong local intra-cortical inhibition makes all ordered patterns unstable and disorganized layouts of orientation preference emerge in the system. The equations are very abstract, modeling the pattern formation describing only one parameter of the neuron tuning curves. Orientation selectivity is assumed to be the only relevant property by construction. Following the principles of universality, the dynamical results of this abstract approach should hold for a larger set of more general models that satisfy the the same symmetry conditions, even if the dynamics are more complicated and not analytically tractable. In this section the previous results are tested in a well known model for the development of oriented receptive fields of simple cells [53].

\subsubsection{Introduction to the model}

The model is based on biological assumptions and was the first to give a dynamical perspective to the Hubel and Wiesel concept of orientation selectivity emerging from selective sampling of ON-center and OFF-center afferents from the lateral geniculate nucleus (LGN). The principle of the model is depicted in figure 2.5.1a) and is introduced as follows:

- The system is composed of a two dimensional layer of V1 neurons, labeled with Latin letters $(x, y, z)$, and a two dimensional layers of LGN neurons, labeled with Greek letters $(\alpha, \beta)$, where for every index in LGN there is an On-center and an Off-center cell.

- The connections between LGN and V1 neurons $S_{O N}(z, \alpha) \geq 0$ and $S_{O F F}(z, \alpha) \geq 0$ are dynamic and changes through Hebbian mechanisms: Correlated activity of the common input leads to a reinforcement of the synaptic weight of the connection at the expense of weakening other 


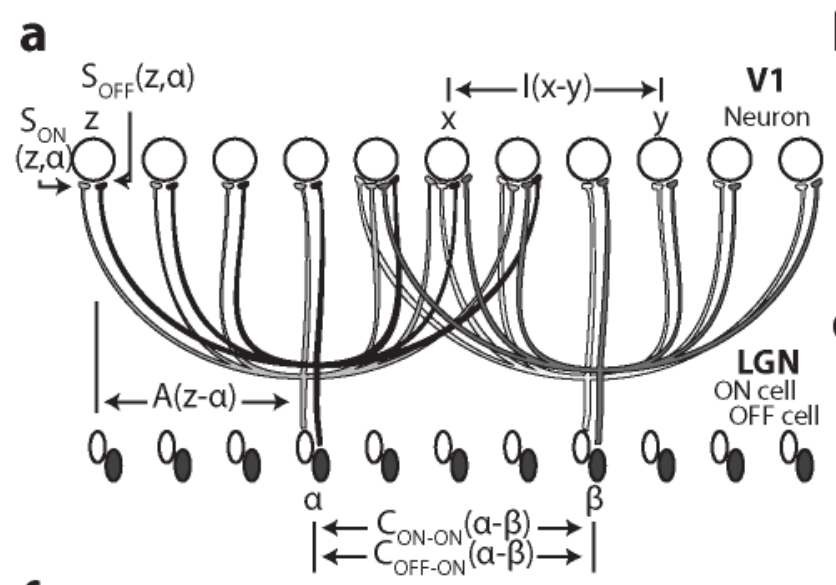

b

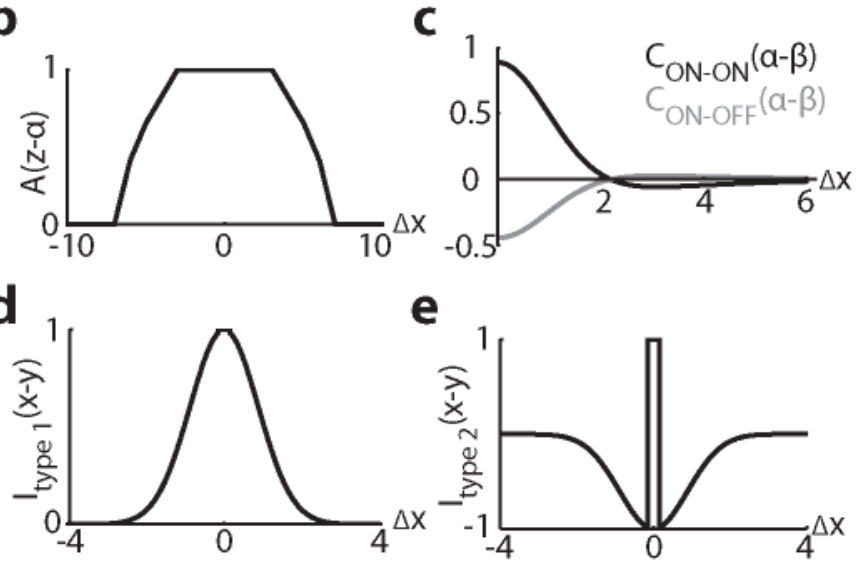

f

Initial condition:
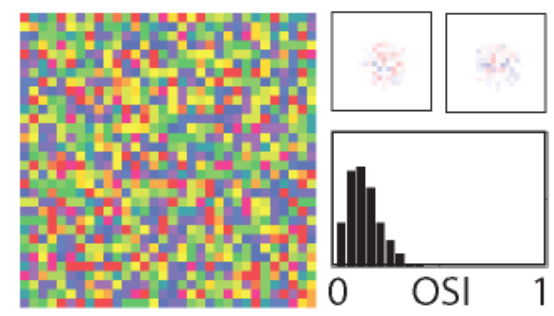

g

After Hebbian dynamics:
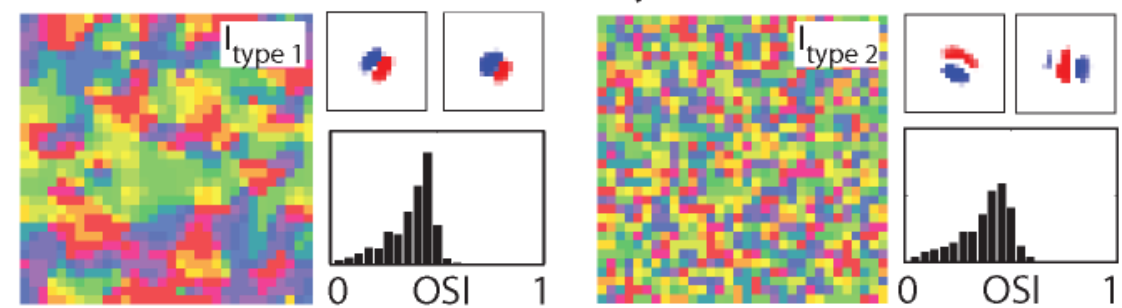

$-1110$

Off $\max =$ On max

Figure 2.5.1: Implementation of strong inhibitory intra-cortical interactions in a correlation based model [53]. a) Sketch of the modeled system, consisting of a 2D layer of V1 neurons $(x, y, z)$ and a 2D layer of LGN neurons $(\alpha, \beta)$, where for every index in LGN there is a pair of On and Off-center cells. Neurons in V1 interact though space dependent intra-cortical interaction $I(x-y)$ and the correlation of the activity of neurons in LGN is given by $C_{O N-O N}(\alpha-\beta), C_{O N-O F F}(\alpha-\beta)$ and $C_{O F F-O F F}(\alpha-\beta)$. The synaptic connections between LGN and V1 neurons is labeled as $S_{O N}(z, \alpha)$ and $S_{O F F}(z, \alpha)$, and is spatially limited by the spread of interactions given by the arbor function $A(z-\alpha)$. Only the connections $S_{O N}(z, \alpha)$ and $S_{O F F}(z, \alpha)$ are dynamic and change through Hebbian mechanisms. b) - e) The interaction functions are plotted with the used parameters in the simulation: b) Arbor function, c) LGN correlation, d) intra-cortical interaction with strong excitation (type I) and e) intra-cortical interaction with strong inhibition (type II). f) - h) Show the simulation results. In each figure the receptive fields of two example neighboring neurons is presented, where red marks an increase in interaction with On cells and blue with Off cells. Below is the histogram of orientation selectivity indices (OSI) in V1, where OSI is defined in equation 2.5.1. The functional layout of V1 is presented where colors represent preferred orientations as marked in the label below. f) The initial condition of the simulations: Connections between V1 and LGN are randomly assigned as $S_{O N / O F F}(x-\alpha)=\left(1+\xi_{1}\right) A(x-\alpha)$ where $\xi_{1} \in \mathcal{N}[0,0.2]$ are normally distributed random numbers. g) - h) Results of simulations after $90 \%$ of the V1 - LGN connections have saturated. In g) the interaction type I was used. Orientation selectivity emerges and the pattern is ordered in space. In h) the interaction type II was used, and although orientation selectivity develops, the spatial organization of orientation preferences is lost. The results therefore confirm the implications of strong intracortical inhibition as derived in the order-parameter model. 
synapses. As an example, the interaction strength between $x$ and $\alpha\left(S_{O N}(x, \alpha)\right)$ is affected by the correlated activity between $\alpha$ and $\beta\left(C_{O N-O N}(\alpha-\beta)\right.$ and $\left.C_{O N-O F F}(\alpha-\beta)\right)$, since $\beta$ is connected to $y$ with weights $S_{O N}(y, \beta)$ and $S_{O F F}(y, \beta)$, and $y$ to $z$ through intracortical interactions $I(x-y)$.

- The range and strength of the interaction between neurons in LGN and V1 is limited by the arbor function $A(z-\alpha)$. The arbor function and the intra-cortical interaction $I(x-y)$ are not dynamic and remain fixed during the simulations.

- With the constructed model, simple receptive fields will form in the neurons in V1 when the On and Off afferents are spatially segregated. This is achieved through a positive correlation between On-On and Off-Off cells, clustering strengthened synapses from similar type of LGN cell together, but a negative correlation between On-Off cells to spatially separate the clusters.

The correlation functions in the LGN will determine the formation of receptive fields, but how the preferred orientations are ordered in space will depend on the intracortical interactions $I(x-y)$. It is important to notice that all the interactions are only distance dependent, and that the dynamics are the same for all neurons in V1 and LGN independent of their index or type. This means that the model satisfies the symmetry conditions that are needed for the previous results to apply. First the original results from Miller will be replicated, where a Mexican hat or a simple positive Gaussian was used for $I(x-y)$. Then strong local inhibition between the neurons in V1 will be implemented to study whether the layout of the pattern changes to a disorganized pattern as predicted by the order-parameter model.

\subsubsection{The equations of the model}

As formulated in the previous section, the strength of the interaction between LGN and V1 cells depends on the correlated activity between LGN neurons and its propagation to V1. The unconstrained learning rule for a synapse between an On-cell $\alpha$ in LGN and a neuron $x$ in V1 is

$$
\frac{d}{d t} \mid \mathrm{U} S^{\mathrm{ON}}(x, \alpha)=\lambda A(x-\alpha) \sum_{y, \beta} I(x-y)\left[C^{\mathrm{ON}, \mathrm{ON}}(\alpha-\beta) S^{\mathrm{ON}}(y, \beta)+C^{\mathrm{ON}, \mathrm{OFF}}(\alpha-\beta) S^{\mathrm{OFF}}(y, \beta)\right]
$$

The learning rule for an Off cell is the same with indices On and Off interchanged. $\lambda$ sets the learning rate and can be varied during the simulations. To include competition between different inputs to a cortical neuron, the strengthening of a synapse to a neuron is combined with a weakening of all other synapses. As in [53] subtractive normalization of the total connection strength will be used. The constrained dynamics are then

$$
\frac{d}{d t} S^{\mathrm{ON}}(x-\alpha)=\frac{d}{d t} S_{\mathrm{U}} S^{\mathrm{ON}}(x-\alpha)-\epsilon(x) A(x-\alpha)
$$

where

$$
\epsilon(x)=\frac{1}{2 \sum_{\beta} A(x-\beta)} \sum_{\beta}\left[\frac{d}{d t}\left|\mathrm{U} S^{\mathrm{ON}}(x, \beta)+\frac{d}{d t}\right| \mathrm{U} S^{\mathrm{OFF}}(x, \beta)\right]
$$


The arbor function between neurons in LGN and V1 is constructed to mimic the physical overlap of the spread of LGN axons and V1 dendrites. Defining $\operatorname{Circ}\left(r_{A}, x\right)$ as a circle of radius $r_{A}$ centered at the neuron $x$ in V1 and $\operatorname{Circ}\left(c_{A} r_{A}, \alpha\right)$ a circle centered at the neuron $\alpha$ in LGN, the arbor function is the overlap of both circles

$$
A(x-\alpha)=\operatorname{Circ}\left(r_{A}, x\right) \cap \operatorname{Circ}\left(c_{A} r_{A}, \alpha\right)
$$

The correlation between neurons in LGN of the same type is defined to have a Mexican hat shape

$$
\mathrm{C}^{\mathrm{ON}, \mathrm{ON}}=C^{\mathrm{OFF}, \mathrm{OFF}}=e^{-\frac{|x|^{2}}{\sigma_{c}^{2}}}-\frac{1}{\gamma_{c}^{2}} e^{-\frac{|x|^{2}}{\gamma_{c}^{2} \sigma_{c}^{2}}}
$$

The Mexican hat is a plausible function for the correlation because a stimulus in the photoreceptor that excites the bright/dark center region of one cell will be part of the surround of neurons a short distance apart. If the neuron is of the same center-surround class, the neighboring neuron will be anti-correlated. For opposite cell types, the correlation is scaled by half and inverted

$$
\mathrm{C}^{\mathrm{OFF}, \mathrm{ON}}=-\frac{1}{2} \mathrm{C}^{\mathrm{ON}, \mathrm{ON}}
$$

Finally two types of intra-cortical interactions are defined. Type I follows the steps of the original paper, writing the interaction as a positive (excitatory) Gaussian

$$
I_{\text {typeI }}(x-y)=e^{-\frac{|x-y|^{2}}{\sigma_{I}^{2}}}
$$

Type II flips the strength of the interaction using a negative (inhibitory) Gaussian

$$
I_{\text {typeII }}(x-y)=a(x-y) e^{-\frac{|x-y|^{2}}{\sigma_{I}^{2}}}, a(0)=1, a(x \neq 0)=-1
$$

At $\Delta x=0$ the interaction is set to 1 such that selectivity in the receptive field can emerge. Otherwise the synapses between neurons in V1 and LGN are not strengthened. The value of $I(0)$ plays the same role as the bifurcation parameter $r$ in the order-parameter model.

\subsubsection{Simulation results}

Following the procedure in [53], the equations were simulated in a system of $32 \times 32$ neurons in V1 and two $32 \times 32$ On and Off layers in LGN with periodic boundary conditions. The used parameters of the interaction functions are $r_{A}=13, c_{A}=0.5, \sigma_{c}=2, \gamma_{c}=3$, and $\sigma_{I}=3$. Figure 2.5.1b) shows the arbor function, where distance is given in units of the separation between two nearest neighbors. Figure 2.5.1c) shows the correlation between LGN neurons with this choice of parameters . Figure 2.5.1d) and e) show the intra-cortical interactions type I and type II respectively. $\lambda$ was set such that the standard deviation of the synaptic changes was 0.01 in every integration step, and was bounded by $\lambda_{\min }=0.01$. Connections that saturate at $S^{\mathrm{ON} / \mathrm{OFF}}(x-\alpha)=0$ or $S^{\mathrm{ON} / \mathrm{OFF}}(x-\alpha)=s_{\max } A(x-\alpha)$ with $s_{\max }=4$ are frozen for the rest of the simulation. The equation is numerically solved with a three step method until $90 \%$ of the connections have reached their maximum or minimum values. 
The initial conditions are depicted in figure 2.5.1f). The strength of the synapses is initialized with $S^{\mathrm{ON} / O F F}(x-\alpha)=\left(1+\xi_{1}\right) A(x-\alpha)$ where $\xi_{1} \in \mathcal{N}[0,0.2]$ are normally distributed random numbers. The receptive field of a neuron $x$ in V1 will be determined by the subtraction of its On and Off afferents for every point $\alpha$ in LGN. In the figure the receptive field of two example neighboring neurons is shown, where red marks a surplus of On input and blue of Off input. For such initial conditions, the receptive fields also exhibit random fluctuations around zero. The preferred orientation of the neurons in V1 is defined as the angle where the presentation of a rotated sine grating to the receptive field gives the maximum response. Numerically this is obtained by calculating the two dimensional Fourier transform of the receptive field, finding the mode $\mathbf{k}$ with highest power and extracting its orientation, $\theta=\frac{1}{2} \arctan \left(\frac{k_{y}}{k_{x}}\right)$. Since the receptive fields are random in the initial condition, the layout of orientation preference is also random. Using the value of the maximum response $R\left(\theta_{n}\right)$ for $n$ orientations in the interval 0 to 180, independent of their spatial frequency (i.e. the magnitude of the mode k), the orientation selectivity index (OSI) of the neuron is defined as the magnitude of the vector sum of the response divided by the total response

$$
\text { OSI }=\frac{\left|\prod_{i=1}^{n} R\left(\theta_{n}\right) e^{i 2 \theta_{n}}\right|}{\sum_{i=1}^{n} R\left(\theta_{n}\right)}
$$

The value of the OSI is bounded by $[0,1]$. When the response is very strong to only one orientation the value approaches 1 , and when the magnitude of the response is independent of the angle $\theta$ the value approaches 0 . Because the receptive fields are linear and of finite size, the neuron response to any given orientation is never zero. This means that the values of OSI that are obtained are not as high as in biological tuning curves, even if the response has a perfect cosine shape. In figure 2.5.1f) a histogram of the selectivities of the initial condition is given. The values are low with a peak close to zero.

Figure 2.5.1g) shows the results of a simulation using the intra-cortical interaction of type I, i.e. with strong lateral excitation. After $90 \%$ of the connections have saturated, receptive fields with segregated On and Off inputs are generated. The receptive fields have an increase orientation selectivity, as is shown in the OSI histogram. As mentioned high values of OSI are not reached, but the distribution skews towards higher values compared to the initial condition. The preferred orientation of the neurons also cluster in space, where neurons with a similar orientation are closer to each other and with smooth transitions between the orientation of neighboring neurons. This replicates the results obtained by Miller. The resulting pattern does not exhibit all spatial characteristics observed in species with maps that are reproduced by the order-parameter model but qualitatively reproduces a spatially continuous organization disrupted by pinwheels. A complete analysis of the organized pattern that emerge for this type of interaction is given in the original paper [53].

In figure 2.5.1h) the type II interaction is implemented, i.e. strong intra-cortical inhibition. According to the results of the order-parameter model, this type of interaction should make ordered solutions unstable, but orientation selectivity is still expected to emerge from the system. The two example neighboring receptive fields show that the On and Off inputs are segregated and oriented in space, although their orientation is very different. According to the OSI histogram the cortical neurons increase their orientation selectivity, similar to the case of excitatory interaction (type I). But in contrast to the results with excitatory interaction, the preferred orientations are disordered in space. With this kind of interaction, the neurons develop orientation selectivity, but they are embedded in an interspersed pattern. 
The results of the implementation of intra-cortical inhibition in this well established model for the development of orientation selectivity demonstrate the generality of the conclusions drawn from the order-parameter model. This confirms the theoretical expectation that simple idealized models that are analytically tractable can help to understand the dynamical principles of other more detailed and biologically realistic approaches. 


\section{Chapter 3}

\section{Model for binocular neurons}

It was recently shown that in binocular neurons of mice the preferred orientations for stimuli presented to each eye are not matched at the onset of vision but become matched only later in development after visual experience [127]. As shown in figure 3.0.1a, at P20-23 the difference in orientation preference between the tuning of inputs from each eye is broad. By P31-P36 the difference has decreased and is maintained until adulthood in P60-P90. The matching of orientation agrees with the overall characteristics of plasticity in the visual cortex, where dark rearing prologues the critical window for the matching and monocular deprivation impairs the matching of the tuning only during the critical period. For normal cortical development these results showed for the first time how activity-dependent mechanisms change the tuning of the neurons to improve response properties.

This finding poses new questions about the nature and dynamics of activity-dependent mechanisms. Every neuron has two sources of input that have to be dynamically regulated to have both orientation preferences matched: The interactions from the LGN, that are eye specific, and the interactions inside the cortex, where no evidence of input-eye specific connections has been reported. Internal mechanism can make the neuron sample from LGN fibers to match the orientation of the receptive field to both eyes, but the interactions with other neurons in the cortex can pull the matching apart by making the neuron resemble the tuning of its intracortical inputs. This effect would be specially strong in the case of interspersed orientation arrangements like in the rodent, where close-by neurons can have a very dissimilar orientation preferences.

In this chapter order-parameter models are generalized to include separate tuning in each eye. The tuning of each neuron $z_{i}$ is split into $z_{i, l}$ and $z_{i, r}$, as shown in figure 3.0.1b, where colors represent the preferred orientation. The tuning to each eye is independently coupled internally for every neuron. Both tuning parameters of a neuron are modified depending on a combination the tuning parameters of neighboring neurons through intra-cortical interactions. The shape of the intra-cortical interactions to use are similar to those described for the monocular model. For simplicity only unselective interactions will be included (see figure 3.0.1c). The derived model is abstract, but as long as the orderparameter approach is appropriate, it will provide a complete list of possibilities for the matching dynamics. Necessary conditions to reproduce the experimental results can then be used to identify a class of candidate models that can be used to predict further features of matching dynamics.

In this part of the thesis first, in the absence of intracortical interaction, the internal inter-eye coupling terms necessary to increase the matching between $z_{i, l}$ and $z_{i, r}$ after the emergence of selectivity are 
a

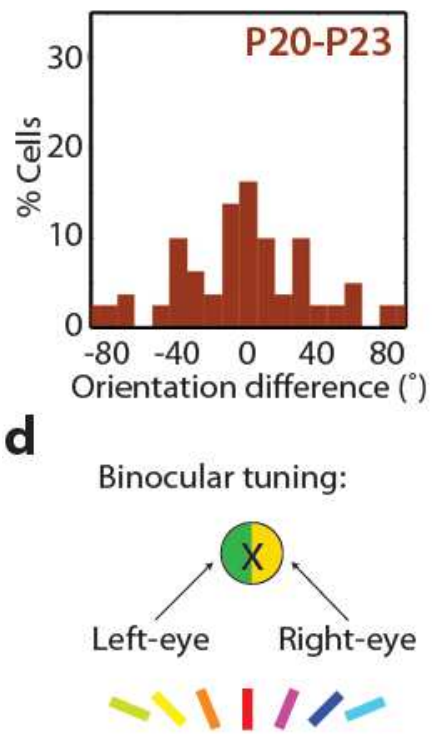

b

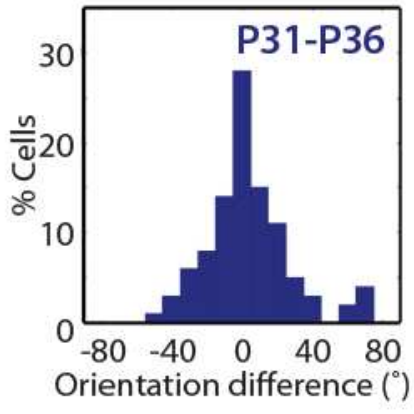

e

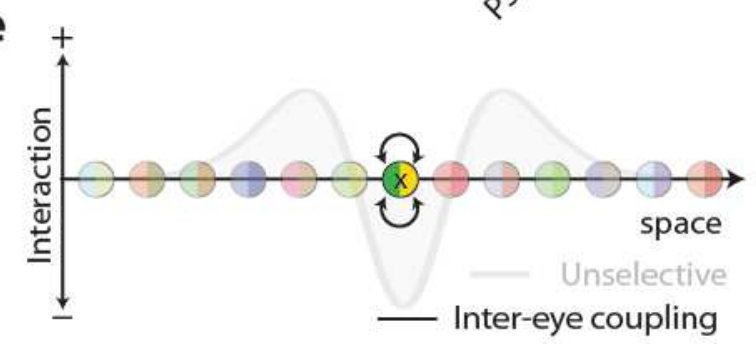

Figure 3.0.1: Binocular neurons in the developing visual cortex of mice match the orientation preference for inputs from each eye through activity dependent mechanisms. a) Soon after eye opening in P20-P23 orientation selectivity has developed in the binocular neurons, but the difference between the preferred orientation to stimuli coming from each eye is broad. b) In animals in P32-P36 this binocular miss-match is reduced and most neurons encode the same orientation for inputs of each eye. c) Only through visual experience the difference between the tuning is narrowed, as in dark-reared (DR) and monocular deprived (MD) animals in P32-P36 the binocular mismatch is not reduced. Adapted from [127]. d) To expand the model to binocular neurons, each tuning is split in two parts representing the left and the right eye. e) For every neuron in the system both tuning curves interact through internal coupling terms, independent of the lateral connections. For simplicity only unselective intra-cortical interactions are taken into account.

found. Then intracortical interactions that generate disordered arrangements (type III connectivity) are included. It is numerically shown that the increase in order by eye matching and the decrease of order by generating an interspersed orientation layout can be simultaneously achieved with the same dynamical equation.

\subsection{Construction of the model}

The aim is to construct the dynamics for the development of the tuning of inputs from each eye

$$
\begin{aligned}
\partial_{t} z_{i, l} & =\hat{F}_{l}\left[z_{l}, z_{r}\right] \\
\partial_{t} z_{i, r} & =\hat{F}_{r}\left[z_{l}, z_{r}\right]
\end{aligned}
$$

Following the steps of the monocular model, the dynamics of the total tuning of each neuron shouldn't depend on its position or orientation, imposing a translation symmetry with operator $\hat{T}_{\mathbf{y}}$

$$
\hat{F}_{l}\left[\hat{T}_{\mathbf{y}} z_{l}, \hat{T}_{\mathbf{y}} z_{r}\right]=\hat{F}_{l}\left[z_{l}(\mathbf{x}+\mathbf{y}), z_{r}(\mathbf{x}+\mathbf{y})\right]=\hat{T}_{\mathbf{y}} \hat{F}_{l}\left[z_{l}, z_{r}\right]
$$


a rotation symmetry with operator $\hat{R}_{\beta}=\left(\begin{array}{cc}\cos (\beta) & -\sin (\beta) \\ \sin (\beta) & \cos (\beta)\end{array}\right)$

$$
\hat{F}_{l}\left[\hat{R}_{\beta} z_{l}, \hat{R}_{\beta} z_{r}\right]=\hat{R}_{\beta} \hat{F}_{l}\left[z_{l}, z_{r}\right]
$$

and a phase shift symmetry

$$
\hat{F}_{l}\left[e^{i \varphi} z_{l}, e^{i \varphi} z_{r}\right]=e^{i \varphi} \hat{F}_{l}\left[z_{l}, z_{r}\right]
$$

The same symmetries apply to $\hat{F}_{r}$. Furthermore, for each neuron, the dynamics shouldn't depend on which eye each tuning curve is representing, making the dynamics equivariant under eye inversion

$$
\hat{F}_{l}\left[z_{l}, z_{r}\right]=\hat{F}_{r}\left[z_{r}, z_{l}\right]
$$

Finally, if neuron $z_{i}$ is interacting with neuron $z_{j}$, an eye inversion in neuron $j,\left[z_{j, l}, z_{j, r}\right] \rightarrow\left[z_{j, r}, z_{j, l}\right]$, shouldn't have an effect on the dynamics of neuron $i$. This imposes an eye inversion symmetry

$$
\hat{F}_{l}\left[z_{i, l}, z_{i, r}, z_{j, l}, z_{j, r}\right]=\hat{F}_{l}\left[z_{i, l}, z_{i, r}, z_{j, r}, z_{j, l}\right]=\hat{F}_{l}\left[z_{i, r}, z_{i, l}, z_{j, l}, z_{j, r}\right]
$$

and similarly for $\hat{F}_{r}$. This last symmetry represents that the intra-cortical interactions are not selective to the response of the neurons to the stimulation from the left or the right eye.

To search for coupling terms in the dynamical equation satisfying these constraints the most general energy of the system is written down up to fourth order including only terms that are invariant under the symmetries. As a reference, from the monocular model using $\frac{\partial E}{\partial \bar{z}}=-\frac{\partial z}{\partial t}$ the energy is

$$
E=-\sum_{i=1}^{N}\left(r\left|z_{i}\right|^{2}+\bar{z}_{i} \frac{1}{N} \sum_{j=1}^{N} K\left(x_{i}-x_{j}\right) z_{j}-\frac{\left|z_{i}\right|^{4}}{2}\right)
$$

The energy of the binocular model will depend on the representation of each eye. For transparency of the construction the energy will be split in intra-neuron coupling terms and inter-neuron coupling terms.

For the intra-neuron coupling terms, up to fourth order, all the possible ways of combining the left and right eye tuning in each neuron and obtain a phase shift invariant energy are

$$
\begin{array}{llll}
z_{i, l} \bar{z}_{i, r} & \bar{z}_{i, l} \bar{z}_{i, l} z_{i, l} z_{i, r} & \bar{z}_{i, l} \bar{z}_{i, l} z_{i, r} z_{i, r} & \bar{z}_{i, l} \bar{z}_{i, r} z_{i, r} z_{i, r} \\
\bar{z}_{i, l} z_{i, r} & z_{i, l} z_{i, l} z_{i, l} \bar{z}_{i, r} & z_{i, l} \bar{z}_{i, l} \bar{z}_{i, r} z_{i, r} & z_{i, l} \bar{z}_{i, r} \bar{z}_{i, r} z_{i, r} \\
& & z_{i, l} z_{i, l} \bar{z}_{i, r} \bar{z}_{i, r} &
\end{array}
$$

Some of those terms have to be combined in order to satisfy the energy invariance under eye inversion. Parametrizing with $\alpha, \gamma, \delta$ and $\epsilon$ all the combination of terms, the most general intra-neuron coupling energy up to fourth order is 


$$
\begin{aligned}
E_{\text {intra-neuron }}= & -\sum_{i=1}^{N}\left[r\left(\left|z_{i, l}\right|^{2}+\left|z_{i, r}\right|^{2}\right)-\frac{1}{2}\left(\left|z_{i, l}\right|^{4}+\left|z_{i, r}\right|^{4}\right)\right] \\
& -\alpha \sum_{i=1}^{N}\left(z_{i, l} \bar{z}_{i, r}+z_{i, r} \bar{z}_{i, l}\right) \\
& -\gamma \sum_{i=1}^{N}\left(z_{i, r} \bar{z}_{i, l}\left|z_{i, l}\right|^{2}+z_{i, l} \bar{z}_{i, r}\left|z_{i, r}\right|^{2}+\bar{z}_{i, r} z_{i, l}\left|z_{i, l}\right|^{2}+\bar{z}_{i, l} z_{i, r}\left|z_{i, r}\right|^{2}\right) \\
& -\delta \sum_{i=1}^{N}\left(\left|z_{i, l}\right|^{2}\left|z_{i, r}\right|^{2}\right) \\
& -\epsilon \sum_{i=1}^{N}\left(z_{i, l}{ }^{2} \bar{z}_{i, r}{ }^{2}+z_{i, r}{ }^{2} \bar{z}_{i, l}{ }^{2}\right)
\end{aligned}
$$

For the inter-neuron coupling the quadratic terms of the energy that are invariant under phase-shift are easy to enumerate

$$
\begin{array}{llll}
\bar{z}_{i, l} z_{j, l} & \bar{z}_{i, l} z_{j, r} & \bar{z}_{i, r} z_{j, l} & \bar{z}_{i, r} z_{j, r} \\
z_{i, l} \bar{z}_{j, l} & z_{i, l} \bar{z}_{j, r} & z_{i, r} \bar{z}_{j, l} & z_{i, r} \bar{z}_{j, r}
\end{array}
$$

Terms maintaining simultaneous and independent eye inversion symmetry are joined and parametrized with $\beta$

$$
E_{\text {inter-neuron }}^{(2)}=-\beta \sum_{i=1}^{N}\left(\left(\bar{z}_{i, r}+\bar{z}_{i, l}\right) \frac{1}{N} \sum_{j=1}^{N} K\left(x_{i}-x_{j}\right)\left(z_{j, l}+z_{j, r}\right)+\text { c.c }\right)
$$

Since the complex conjugate is obtained by changing the neuron index $i \leftrightarrow j$ and this doesn't modify the energy, both terms are real and equivalent, such that one of them can be dropped.

Inter-neuron coupling terms of fourth order are more abundant. There are 82 ways of combining the left and right tuning of neuron $i$ and $j$ and obtain a phase shift symmetric energy. Simultaneous and independent eye inversion symmetry combines this permutations using 29 parameters, where some of the terms are complex conjugates of each other and have to be joined to make the energy real. Terms were neuron $j$ has combinations of the left and right eye tuning (e.g. $z_{j, l} z_{j, r}$ or $z_{j, l}\left|z_{j, r}\right|^{2}$ ) are not to be included because they lead to a cancellation of inter-neuron interactions with monocular neurons, which is unlikely to happen in the cortex. Examples of possible fourth order terms are

$$
\begin{gathered}
-\sum_{i, j=1}^{N} K\left(x_{i}-x_{j}\right)\left(\left(\bar{z}_{i, r}+\bar{z}_{i, l}\right)\left(z_{j, l}\left|z_{j, l}\right|^{2}+z_{j, r}\left|z_{j, r}\right|^{2}\right)+\text { c.c }\right) \\
-\sum_{i, j=1}^{N} K\left(x_{i}-x_{j}\right)\left(\left(\left|z_{i, r}\right|^{2}+\left|z_{i, l}\right|^{2}\right)\left(\left|z_{j, l}\right|^{2}+\left|z_{j, r}\right|^{2}\right)\right) \\
\quad-\sum_{i, j j=1}^{N} K\left(x_{i}-x_{j}\right)\left(\left(z_{i, r}^{2}+z_{i, l}^{2}\right)\left(\bar{z}_{j, l}^{2}+\bar{z}_{j, r}^{2}\right)\right) \\
-\sum_{i, j=1}^{N} K\left(x_{i}-x_{j}\right)\left(\left(z_{i, l} \bar{z}_{i, r}+\bar{z}_{i, l} z_{i, r}\right)\left(\left|z_{j, l}\right|^{2}+\left|z_{j, r}\right|^{2}\right)\right)
\end{gathered}
$$

In this study the focus is on the role of the intra-neuron interactions in matching the binocular tuning of the neurons, such that only the quadratic inter-neuron energy term is used. The parameter $\beta$ only 
scales the interaction kernel, such that without loss of generality it can be set to $\frac{1}{2}$ to represent an average of the tuning of neuron $j$. Taken together, the dynamics of the tuning for the right eye are

$$
\begin{aligned}
\partial_{t} z_{i, r}= & r z_{i, r}-z_{i, r}\left|z_{i, r}\right|^{2}+\frac{1}{N} \sum_{j=1}^{N} K\left(x_{i}-x_{j}\right)\left(\frac{z_{j, l}+z_{j, r}}{2}\right) \\
& +\alpha z_{i, l}+\gamma\left(2 z_{i, l}\left|z_{i, r}\right|^{2}+z_{i, l}\left|z_{i, l}\right|^{2}+\bar{z}_{i, l} z_{i, r}{ }^{2}\right)+\delta z_{i, r}\left|z_{i, l}\right|^{2}+\epsilon \bar{z}_{i, r} z_{i, l}{ }^{2}
\end{aligned}
$$

The equation for the left eye tuning is obtained by interchanging the indices $r$ and $l$.

\subsection{Stationary solutions and their stability}

Next all inter-neuron interaction terms will be set to zero and the effect of the intra-neuron coupling will be investigated. What are the conditions for the tuning of each eye to become matched? When does the matching occur? Are there other attractor states? To answer these questions, the existence and stability of different solutions to the equations will be analyzed. Since individual neurons are considered the index $i$ will be dropped. If $\left[z_{r 0}, z_{10}\right]$ is a stationary solution to both equations, the linear stability matrix of a general perturbation $\left[z_{r p}, z_{l p}\right]$ is

$$
\partial_{t}\left(\begin{array}{c}
z_{\mathrm{rp}} \\
z_{\mathrm{lp}}
\end{array}\right)=M_{1}\left(\begin{array}{c}
z_{\mathrm{rp}} \\
z_{\mathrm{lp}}
\end{array}\right)+M_{2}\left(\begin{array}{c}
\bar{z}_{\mathrm{rp}} \\
\bar{z}_{\mathrm{lp}}
\end{array}\right)
$$

with

$$
\begin{gathered}
M_{1}=\left(\begin{array}{cc}
r-2\left|z_{\mathrm{r} 0}\right|^{2}+2 \gamma z_{10} \bar{z}_{\mathrm{r} 0}+2 \gamma z_{\mathrm{r} 0} \bar{z}_{10}+\delta\left|z_{10}\right|^{2} & \alpha+2 \gamma\left(\left|z_{\mathrm{r} 0}\right|^{2}+\left|z_{10}\right|^{2}\right)+\delta z_{\mathrm{r} 0} \bar{z}_{10}+2 \epsilon z_{10} \bar{z}_{\mathrm{r} 0} \\
\alpha+2 \gamma\left(\left|z_{\mathrm{r} 0}\right|^{2}+\left|z_{10}\right|^{2}\right)+\delta z_{10} \bar{z}_{\mathrm{r} 0}+2 \epsilon z_{\mathrm{r} 0} \bar{z}_{10} & r-2\left|z_{10}\right|^{2}+2 \gamma z_{\mathrm{r} 0} \bar{z}_{10}+2 \gamma z_{10} \bar{z}_{\mathrm{r} 0}+\delta\left|z_{\mathrm{r} 0}\right|^{2}
\end{array}\right) \\
M_{2}=\left(\begin{array}{cc}
-z_{\mathrm{r} 0}^{2}+2 \gamma z_{10} z_{\mathrm{r} 0}+\epsilon z_{10}^{2} & \gamma z_{10}^{2}+\gamma z_{\mathrm{r} 0}^{2}+\delta z_{10} z_{\mathrm{r} 0} \\
\gamma z_{\mathrm{r} 0}^{2}+\gamma z_{10}^{2}+\delta z_{\mathrm{r} 0} z_{10} & -z_{10}^{2}+2 \gamma z_{\mathrm{r} 0} z_{10}+\epsilon z_{\mathrm{r} 0}^{2}
\end{array}\right)
\end{gathered}
$$

To obtain the growth rate of the perturbation the eigenvalues of $M_{1}$ and $M_{2}$ have to be found simultaneously. For this the system is represented using a $4 x 4$ real matrix

$$
\partial_{t}\left(\begin{array}{c}
z_{r p} \\
z_{l p} \\
\bar{z}_{\mathrm{rp}} \\
\bar{z}_{\mathrm{lp}}
\end{array}\right)=\left(\begin{array}{cc}
M_{1} & M_{2} \\
\bar{M}_{2} & \bar{M}_{1}
\end{array}\right)\left(\begin{array}{c}
z_{r p} \\
z_{l p} \\
\bar{z}_{\mathrm{rp}} \\
\bar{z}_{\mathrm{lp}}
\end{array}\right)
$$

The parameters $\alpha, \gamma, \delta$ and $\epsilon$ determine the existence of a stationary solution $\left[z_{r 0}, z_{l 0}\right]$. To check for its stability the values of the solution have to be inserted in $M_{1}$ and $M_{2}$ in equation 3.2.1 and the eigenvalues of the matrix calculated. The parameters $\alpha, \gamma, \delta$ and $\epsilon$ will then determine if the eigenvalues are negative or positive, meaning that the stationary solution is stable or unstable respectively.

The conditions for the existence and stability of the following solutions will be checked: 
- Unselective state, with both $z_{r 0}=z_{l 0}=0$.

- Monocular orientation selective state, with $z_{r 0}=A e^{i 2 \theta_{r}}$ and $z_{l 0}=0$ (or vice versa).

- Binocular orientation selective state, with $z_{r 0}=A e^{i 2 \theta}$ and $z_{l 0}=A e^{i 2(\theta+\phi)}$, where both selectivities are equal but the preferred orientations are potentially shifted by a phase $\phi$.

\subsubsection{Unselective state}

\section{Conditions for stationarity}

The unselective state $\left[z_{r 0}=0, z_{l 0}=0\right]$ always exists due to the symmetries of the system, independent of the choice of parameters.

\section{Conditions for stability}

To calculate the stability of this stationary state $\left[z_{r 0}=0, z_{l 0}=0\right]$ is inserted in equation 3.2.1 which simplifies to

$$
\partial_{t}\left(\begin{array}{c}
z_{\mathrm{rp}} \\
z_{\mathrm{lp}}
\end{array}\right)=\left(\begin{array}{cc}
r & \alpha \\
\alpha & r
\end{array}\right)\left(\begin{array}{c}
z_{\mathrm{rp}} \\
z_{\mathrm{lp}}
\end{array}\right)
$$

The eigenvalues of the perturbation are

$$
\begin{aligned}
& \lambda_{1}=r+\alpha \\
& \lambda_{2}=r-\alpha
\end{aligned}
$$

As in the monocular model, $r$ is the bifurcation parameter, where for $r>0$ symmetry is spontaneously broken independent of the value of $\alpha$ and orientation selectivity emerges at least for one eye. If $r<0$, the bifurcation is shifted to $|r|<|\alpha|$.

\subsubsection{Monocular orientation selective state}

\section{Conditions for stationarity}

Now solutions will be checked where neurons are orientation selective to the input of one eye only, $\left[z_{r 0}=z_{r}, z_{10}=0\right]$. Inserting this state in the tuning dynamics in equation 3.1.2 and its left eye equivalent leads to

$$
\begin{aligned}
& \partial_{t} z_{r}=\left(r-\left|z_{r}\right|^{2}\right) z_{r} \\
& \partial_{t} z_{l}=\left(\alpha+\gamma\left|z_{r}\right|^{2}\right) z_{r}
\end{aligned}
$$


Therefore, the conditions for this solution to be a stationary are $\left|z_{r}\right|^{2}=r$ and either $\alpha=\gamma=0$ or $r=-\frac{\alpha}{\gamma}$. For the model to produce monocular selective neurons either some intra-eye coupling terms are not to be included, or they need to be balanced depending on the selectivity of the monocular tuning. This requirement for parameter tuning would be relaxed if we allow the selectivities for the "weak" eye to be non-zero but small, a case that we do not consider in the following for simplicity.

\section{Conditions for stability}

To calculate the stability of the monocular selective state $\left[z_{r 0}=z_{r}, z_{l 0}=0\right]$ with $\left|z_{r}\right|^{2}=r$, the state is inserted in the dynamics for a general perturbation in equation 3.2.1. The eigenvalues of the perturbation are

$$
\begin{aligned}
& \lambda_{1,2}=\frac{1}{2}\left(r(1+\delta-\epsilon) \pm \sqrt{\left(4 \gamma^{2}+(1+\delta-\epsilon)^{2}\right) r^{2}+4 \alpha(\alpha+2 r \gamma)}\right) \\
& \lambda_{3,4}=\frac{1}{2}\left(r(-1+\delta+\epsilon) \pm \sqrt{\left(36 \gamma^{2}+(3+\delta+\epsilon)^{2}\right) r^{2}+4 \alpha(\alpha+6 r \gamma)}\right)
\end{aligned}
$$

- With the first choice of parameters for stationarity, $\alpha=\gamma=0$ the conditions to have all $\lambda_{1,2,3,4} \leq$ 0 and therefore the solution stable are

$$
\begin{aligned}
& \delta-\epsilon \leq-1 \\
& \delta+\epsilon \leq-1
\end{aligned}
$$

This means that the stability of this solution requires nonzero coupling between the eyes. Furthermore, since adding both conditions leads to $\delta \leq 1$, weak coupling makes the monocular orientation selectivity unstable. The larger $\delta$, the larger the range of $\epsilon$ where the solution is stable.

- With the second choice of parameters, $\left|z_{r}\right|^{2}=r=-\frac{\alpha}{\gamma}$, the conditions to have all $\lambda_{1,2,3,4} \leq 0$ are

$$
\begin{aligned}
\delta-\epsilon & \leq-1 \\
-1+\delta+\epsilon \pm \sqrt{(3+\delta+\epsilon)^{2}+(4 \gamma)^{2}} & \leq 0
\end{aligned}
$$

This is an extended case of the previous condition where at least 3 of the 4 parameters are non-zero and strong coupling is necessary.

\subsubsection{Binocular orientation selective state}

\section{Conditions for stationarity}

The third solution to examine are neurons with equal selectivity $A_{r}=A_{l}$ in both eyes but potentially different orientation preferences, $\left[z_{r}=A e^{i 2 \theta_{r}}, z_{l}=A e^{i 2 \theta_{l}}\right]$. Furthermore, since the interest is mostly 
in the difference between the preferred orientations, the notation $\left[z_{r}=A e^{i 2 \theta}, z_{l}=A e^{i 2(\theta+\phi)}\right]$ can be used. Inserting this into the tuning dynamics in equation 3.1.2 and simplifying after making the amplitude stationary, $\frac{\partial A}{\partial t}=0$ leads to

$$
\begin{aligned}
\partial_{t} i 2 \theta & =r+\alpha e^{i 2 \phi}+|A|^{2}\left(-1+\gamma\left(3 e^{i 2 \phi}+e^{-i 2 \phi}\right)+\delta+\epsilon e^{i 4 \phi}\right) \\
\partial_{t} i 2(\theta+\phi) & =r+\alpha e^{-i 2 \phi}+|A|^{2}\left(-1+\gamma\left(3 e^{-i 2 \phi}+e^{i 2 \phi}\right)+\delta+\epsilon e^{-i 4 \phi}\right)
\end{aligned}
$$

For this solution to be stationary, both $\partial_{t} i 2 \theta \equiv 0$ and $\partial_{t} i 2(\theta+\phi) \equiv 0$. Each equation determines the selectivity the neuron must have for this solution to be stationary for a given orientation $\theta$ and orientation offset $\phi$

$$
|A|^{2}=\frac{r+\alpha e^{i 2 \phi}}{1-\gamma\left(3 e^{i 2 \phi}+e^{-i 2 \phi}\right)-\delta-\epsilon e^{i 4 \phi}}
$$

The conditions on the parameters are therefore that the right hand side of equation 3.2.2 is real and positive.

Setting both dynamical equations to zero and subtracting them the conditions for the orientation offset to be stationary are obtained

$$
-\alpha \sin (2 \phi)-2 \gamma|A|^{2} \sin (2 \phi)-\epsilon|A|^{2} \sin (4 \phi)=0
$$

With no restriction on the choice of interaction parameters, the orientation difference between the neurons in the stationary state are $\left\{\phi=0, \phi= \pm \frac{\pi}{2}\right\}$. If the choice of parameters includes $\alpha=\gamma=0$, the stationary orientation differences extend to $\left\{\phi=0, \phi= \pm \frac{\pi}{2}, \phi= \pm \frac{\pi}{4}\right\}$. If all coupling parameters are set to zero and $r>0$, the binocular selective state is stable independent of the orientation shift $\phi$, since in this case the tuning curves are not coupled and the orientation offset is irrelevant. Similarly, when only $\delta \neq 0$ the binocular state is stable, but $\phi$ is marginal since it doesn't play a role in the orientation dynamics. Other solutions for $\phi$ with non-zero parameters are not possible since the right hand side of equation 3.2.2 would not be real. An orientation offset $\phi=0$ means that the tuning curves are matched, $\phi= \pm \frac{\pi}{2}$ means that the preferred orientation of inputs from each eye is orthogonal, and $\phi= \pm \frac{\pi}{4}$ means oblique preferred orientations between the eyes.

\section{Conditions for stability}

To calculate the stability of binocular states with orientation offset $\phi$ and selectivity $|A|^{2}$, the solution $\left[z_{r}=A e^{i 2 \theta}, z_{l}=A e^{i 2(\theta+\phi)}\right]$ is inserted in the general stability matrix in equation 3.2.1 and the conditions on the parameters for the eigenvalues to be negative are calculated. Each case will be treated separately.

- Orientation offset $\phi=0$

The stationary selectivity for this solution in equation 3.2.2 is $|A|^{2}=\frac{r+\alpha}{1-4 \gamma-\delta-\epsilon}$. The eigenvalues of the perturbation matrix are 


$$
\begin{aligned}
& \lambda_{1}=0 \\
& \lambda_{2}=-2(r+\alpha) \\
& \lambda_{3}=\frac{-2(r(1+2 \gamma+\delta+\epsilon)-2 \alpha(-1+\gamma))}{1-4 \gamma-\delta-\epsilon} \\
& \lambda_{4}=\frac{-2(2 r(\gamma+\epsilon)+\alpha(1-2 \gamma-\delta+\epsilon))}{1-4 \gamma-\delta-\epsilon}
\end{aligned}
$$

The first eigenvalue is zero because it represents a perturbation along the phase shift symmetry. It will appear in the eigenvalues for all other orientation offsets. For the second eigenvalue to be negative, the condition $r+\alpha>0$ is imposed. This also imposes that the denominator of the selectivity must be positive, such that $1-4 \gamma-\delta-\epsilon>0$. This simplifies the conditions for $\lambda_{3,4} \leq 0$ to $-r(1+2 \gamma+\delta+$ $\epsilon)+2 \alpha(-1+\gamma) \leq 0$ and $-2 r(\gamma+\epsilon)-\alpha(1-2 \gamma-\delta+\epsilon) \leq 0$.

- Orientation offset $\phi= \pm \frac{\pi}{2}$

The stationary selectivity in this case is $|A|^{2}=\frac{r-\alpha}{1+4 \gamma-\delta-\epsilon}$. The eigenvalues of the perturbation matrix are

$$
\begin{aligned}
& \lambda_{1}=0 \\
& \lambda_{2}=-2(r-\alpha) \\
& \lambda_{3}=2 r-\frac{4(r-\alpha)(1+\gamma)}{1+4 \gamma-\delta-\epsilon} \\
& \lambda_{4}=\frac{2(2 r(\gamma-\epsilon)+\alpha(1+2 \gamma-\delta+\epsilon))}{1+4 \gamma-\delta-\epsilon}
\end{aligned}
$$

As with the previous case, to have the second eigenvalue negative the condition $r-\alpha>0$ is imposed and therefore the denominator of the amplitude must also be positive, imposing $1+4 \gamma-\delta-\epsilon>0$. The other conditions to have negative eigenvalues simplify to $r(2 \gamma-(1+\delta+\epsilon))+2 \alpha(1+\gamma) \leq 0$ and $2 r(\gamma-\epsilon)+\alpha(1+2 \gamma-\delta+\epsilon) \leq 0$

- Orientation offset $\phi= \pm \frac{\pi}{4}$

For $\phi= \pm \frac{\pi}{4}$ to be a stationary solution the condition $\alpha=\gamma=0$ has to be satisfied. The selectivity of the state by inserting in equation 3.2.2 is $|A|^{2}=\frac{r}{1-\delta+\epsilon}$. The eigenvalues of the perturbation are

$$
\begin{aligned}
& \lambda_{1}=0 \\
& \lambda_{2}=-2 r \\
& \lambda_{3}=\frac{4 r \epsilon}{1-\delta+\epsilon} \\
& \lambda_{4}=\frac{r(-2-\delta+\epsilon)}{1-\delta+\epsilon}
\end{aligned}
$$

Since the second eigenvalue imposes $r>0$, the condition $1-\delta+\epsilon>0$ must be satisfied to have the selectivity positive. The other conditions to have negative eigenvalues simplify to $\epsilon \leq 0$ and $-\delta+\epsilon \leq 2$. Two conditions can be combined into $-1<-\delta+\epsilon \leq 2$. 


\subsection{Single parameter conditions for late orientation preference matching}

A model where the neurons develop orientation selectivity for both eyes with matched orientation can be constructed with many combinations of parameters. In this work priority is given to simple models with a small amount of parameters. Therefore, solutions on the axes of the model parameter space will be calculated, i.e. only one non-zero parameter at the time will be used. For simplicity $r>0$ as in the monocular model will be assumed. For each solution the single parameter conditions for stability are as follows:

- Unselective state:

- Never stable with $r>0$

- Monocular orientation selective state:

$\cdot \delta \leq-1$

- Binocular orientation selective state with $\phi=0$ :

$\cdot \alpha>0$

$\cdot 0<\gamma<\frac{1}{4}$

. $1<\delta<1$ ( $\phi$ is marginal, so $\phi=0$ is stable )

. $0<\epsilon<1$

- Binocular orientation selective state with $\phi= \pm \frac{\pi}{2}$ :

$\cdot \alpha<0$

$-\frac{1}{4}<\gamma \leq 0$

- $1<\delta<1$ ( $\phi$ is marginal, so $\phi= \pm \frac{\pi}{2}$ is stable )

$\cdot 0<\epsilon<1$

- Binocular orientation selective state with $\phi= \pm \frac{\pi}{4}$ :

$\cdot-1<\epsilon<0$

. $1<\delta<1$ ( $\phi$ is marginal, so $\phi= \pm \frac{\pi}{4}$ is stable $)$

These results are summarized in figure 3.3.1a), where the change of the stable solution as each single parameter is varied and the others are set to zero is plotted. The binocular selective state with orientation offset $\phi=0$ can be achieved in models using every single parameter independently, but in some of those cases the solution is shared with other stable offsets. The model with $-1<\delta<1$ is discarded because the offset $\phi$ is marginal. The model with $0<\epsilon<1$ is also discarded because the stability is shared with the offset $\phi= \pm \frac{\pi}{2}$. Finally, models with $\alpha>0$ have the coupling between the tuning of each eye in the linear term, so although matched neurons are the only stable solution, this 
happens at the same time as orientation selectivity is developing. Since this contradicts the experimental findings reported at the beginning of this part of the thesis, models with this parameter are also discarded. Taken together, the only single parameter model for late orientation matching is

$$
\partial_{t} z_{i, r}=r z_{i, r}-z_{i, r}\left|z_{i, r}\right|^{2}+\gamma\left(2 z_{i, l}\left|z_{i, r}\right|^{2}+z_{i, l}\left|z_{i, l}\right|^{2}+\bar{z}_{i, l} z_{i, r}{ }^{2}\right) \text { with } 0<\gamma<\frac{1}{4}
$$

The validity of the previous results on the single parameter models are checked numerically. For this the inter-neuron interactions are set to zero and equation 3.1.2 is numerically solved. Since for individual neurons the dynamics in equation 3.1.2 is independent of their distribution in space, the system is solved with a vector of 1024 sets of tuning pairs $\left[z_{i, l}, z_{i, r}\right]$. The bifurcation parameter is set to $r=0.1$ and the system is simulated for $100 \tau$, where $\tau=\frac{1}{r}$ sets the timescale for emergence from the unselective state. The dynamical equation is integrated using a 4-5th order Runge-Kutta-Fehlberg method, where the error of the integration is kept below $10^{-2} r$. The initial condition are neurons with randomly distributed tuning $z_{i, r}=\frac{1}{100} \sqrt{r \xi_{1}} e^{i 2 \xi_{2}}$ and $z_{i, l}=\frac{1}{100} \sqrt{r \xi_{3}} e^{i 2 \xi_{4}}$, where $\xi_{1,3} \in \mathcal{U}[0,1]$ and $\xi_{2,4} \in \mathcal{U}[0, \pi]$ are sequences of uniformly distributed random numbers. Figure 3.3.1b) show the results of the simulation for different choices of interaction parameters. In each figure for different time points of the simulation the mean selectivity of the tuning of each eye is plotted at the left and the distribution of the absolute orientation offset is plotted at the right. The selectivity is normalized by the maximum selectivity achieved by any tuning after $100 \tau$.

The simulations validate the analytical results. As mentioned with $\alpha>0$ the matching occurs before selectivity develops. For the monocular selective state with $\delta \leq-1$ the mean selectivity for each eye is half of the maximum, meaning that approximately half of the neurons are tuned only to one eye. This can be read from the mean absolute difference in selectivities of left and right tuning, which reaches the maximum value. In this parameter regime the selectivity first increases in both tuning curves, but then the coupling becomes strong and one of the selectivities is reduced to zero. These results are representative examples; different values for the parameters can be chosen leading to the same behavior as long as parameters are in the calculated ranges.

\subsection{Including spatial interaction}

Using the analytical and simulation results without intra-cortical interactions, equation 3.1.2 reduces to

$$
\partial_{t} z_{i, r}=r z_{i, r}+\frac{1}{N} \sum_{j} K\left(x_{i}-x_{j}\right)\left(\frac{z_{j, l}+z_{j, r}}{2}\right)-z_{i, r}\left|z_{i, r}\right|^{2}+\gamma\left(2 z_{i, l}\left|z_{i, r}\right|^{2}+z_{i, l}\left|z_{i, l}\right|^{2}+\bar{z}_{i, l} z_{i, r}^{2}\right)
$$

The unselective interaction kernel defined in the monocular model will be used,

$$
K\left(x_{i}-x_{j}\right)=\frac{S}{\sqrt{2 \pi} W \sigma} e^{-\frac{\left(x_{i}-x_{j}\right)^{2}}{2 W^{2} \sigma^{2}}}-\frac{1-S}{\sqrt{2 \pi}(1-W) \sigma} e^{-\frac{\left(x_{i}-x_{j}\right)^{2}}{2(1-W)^{2} \sigma^{2}}}
$$

where $S$ and $1-S$ describe the strength of excitation and inhibition respectively and $W \sigma$ and $(1-$ $W) \sigma$ their range. Using this equation, the coexistence of eye matching and disordered layouts will 
a

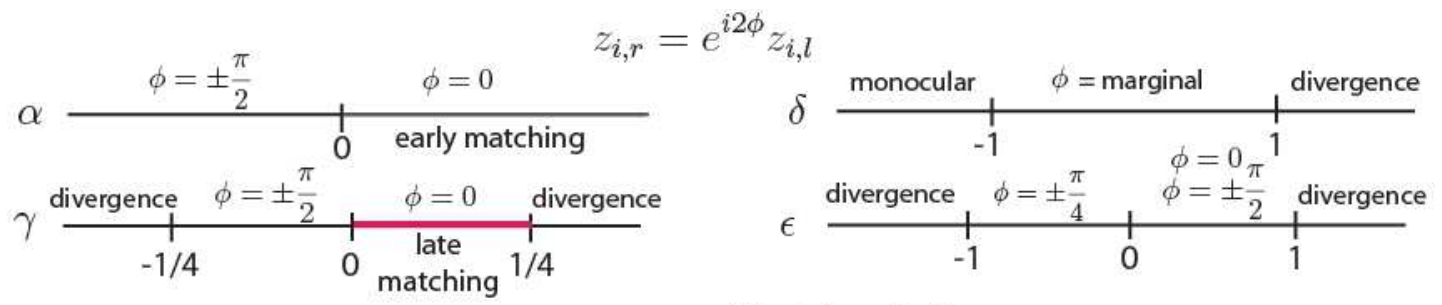

b
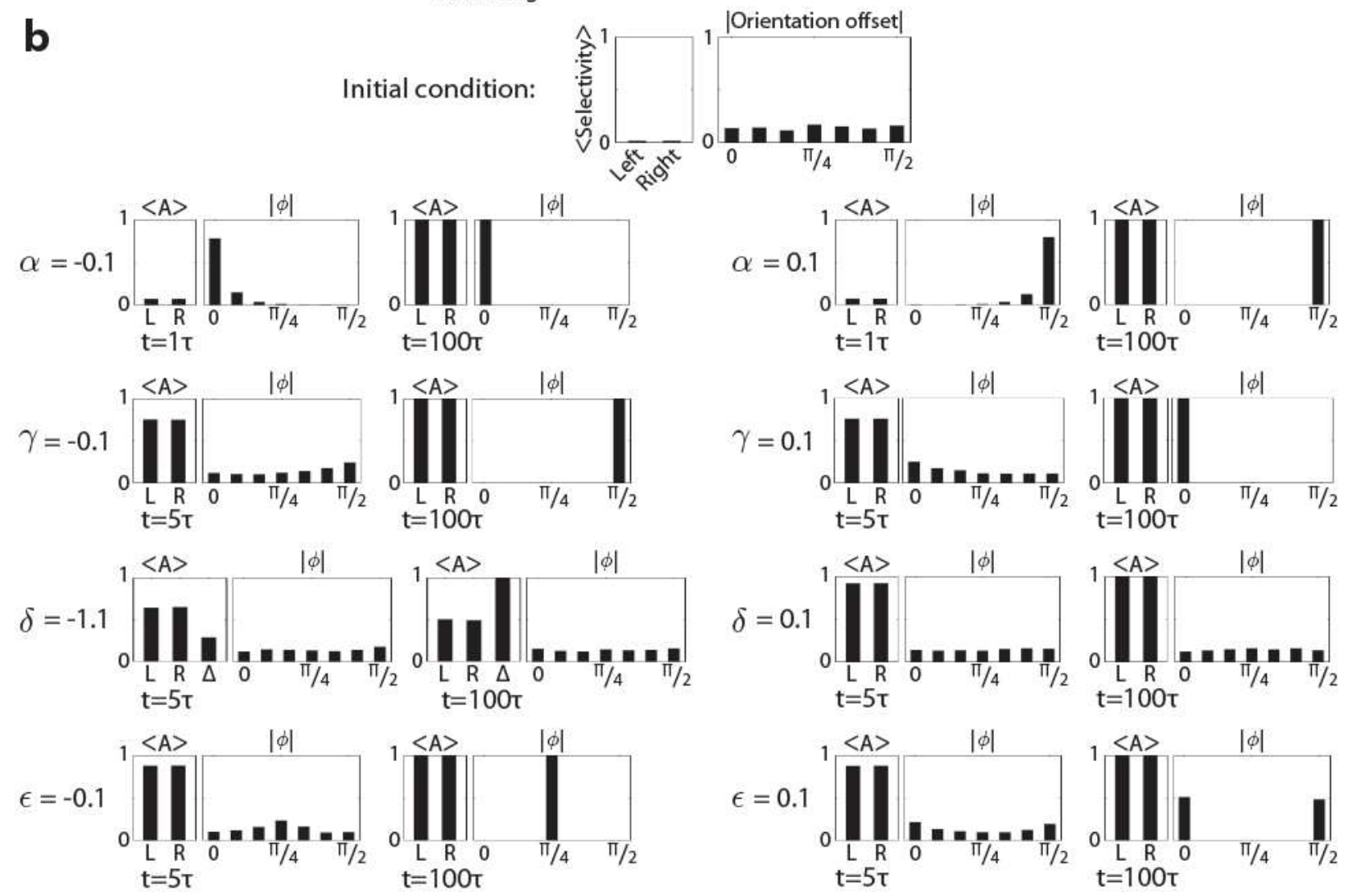

Figure 3.3.1: Results of the binocular model without intra-cortical interactions. a) The analytical results for single parameter models are presented graphically. As each parameter is individually varied and the rest is set to zero different solutions become stable. The only parameter regime where late binocular selectivity matching occurs and all other solutions are unstable is with $0<\gamma<\frac{1}{4}$, marked in red in the figure. b) Simulation of a system of 1024 neurons with $r=0.1$ and different choices of interaction parameters. For two time points of the simulation the mean selectivity of the left eye and right eye tuning is plotted, where the selectivity is normalized by the maximum selectivity achieved by the neurons in the simulation. For $\delta=-1.1$ the mean absolute difference between left and right eye tunings is also plotted. Next to it is a histogram of the fraction of orientation offsets between the left and right eye tunings. The simulations validate the analytical results. 
be numerically investigated. A grid of $128 \times 128$ neurons is simulated in a box of length $L=2 \pi \sigma$ with periodic boundary conditions. The numerical integration method and initial conditions are the same as in the simulations without intra-cortical interactions. Using the parameters $r=0.1, \sigma=1$, $S=0.25, W=0.75$ and $\gamma=0.7 r$ the system is developed for $1000 \tau$. The results are not significantly modified with a different choice of $S, W$ and $\gamma$ as long as the interactions meet the requirements to generate an interspersed pattern and $0<\gamma<\frac{1}{4}$ to obtain late orientation matching.

Figure 3.4.1 shows the results of an example simulation. In figure a) the development of the selectivity of the left eye tuning in gray and right eye tuning in black for one example neuron is shown. In both tuning curves orientation selectivity emerges from the unselective state after $1 \tau$ and then saturates, making it a binocular neuron. In figure b) the development of the orientation for the left and right eye is plotted, where the initial orientation of the left neuron is defined as zero. Comparing both figures a) and b) it can be observed that the matching of the preferred orientation for this neuron happens only after the onset of selectivity. Figure c) shows the mean orientation difference between the neurons of the system in time. It shows that the offset is only reduced once the selectivity has developed. Taken together, figure a) to c) show that the late matching of the tuning curves also takes place when intra-cortical interactions are present.

Figure 3.4.1d ) shows the orientation layout at three time points of the simulation. The tuning at the left and right eye inputs are labeled with colors, where the preferred orientation is encoded with hue and the selectivity with brightness. In the initial condition the neurons have a low selectivity and the orientation for each eye is random. At $10 \tau$ the selectivity has increased, but the preferred orientations remain different from each other. Only later in development the orientations are matched, but no spatial order in the orientation layout emerges. This shows numerically that the matching of the tuning for inputs to both eyes is not impaired by the presence of an interspersed pattern.

The detailed spatial structure of the interspersed pattern as seen in the monocular model doesn't change, but compared to it the dynamics are very different. Figure 3.4.1e) shows in red the average absolute change of the orientation of neurons in the monocular model with respect to the initial condition. Since the initial condition is already disordered, neurons only change their orientations by a few degrees (see chapter 4). In the binocular model on the other hand, neurons have to change their tuning much more because the preferred orientations have to be matched and the interspersed pattern kept simultaneously. In the figure this translates into a much higher mean absolute change in orientation, which starts after the onset of selectivity and approaches 22.5 degrees, which is the mean shift the neurons have to undergo to match an average difference of 45 degrees between two randomly drawn orientations.

That the interspersed pattern is maintained after such a high orientation change is an argument that the layout is determined dynamically by activity dependent mechanisms and is not predefined by genetic or structural cues [116, 117].

\subsection{Predictions in a PSD-95 knock-out experiment}

The model with parameters $0<\gamma<\frac{1}{4}$ and $\beta=1$ reproduces the experimental results described in the introduction. Can the model also predict the outcome of a proposed experiment?

Postsynaptic density protein 95 (PSD-95) is the best characterized of the synaptic PDZ proteins [151]. 
a

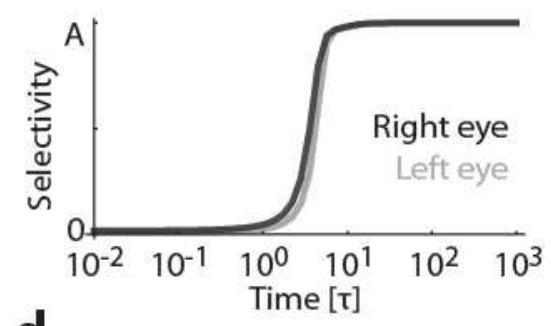

d

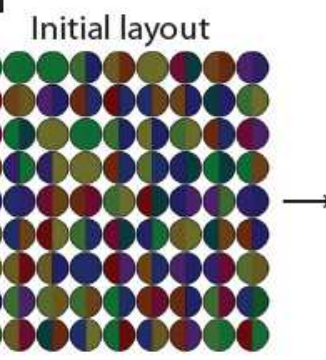

b

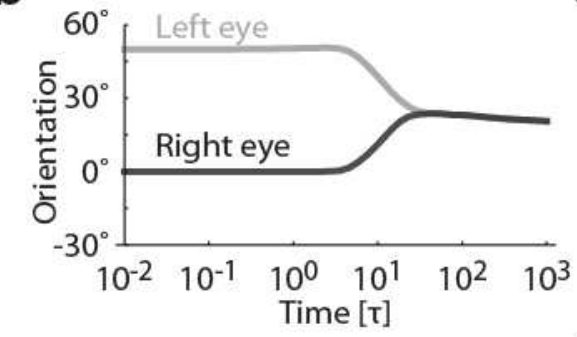

C

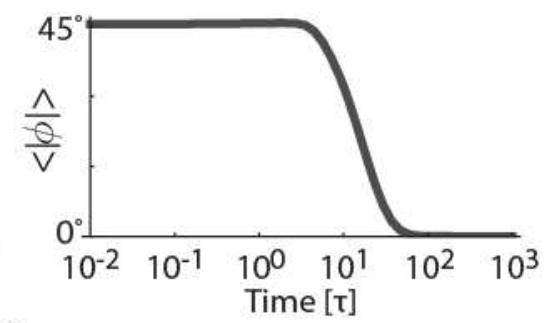

e

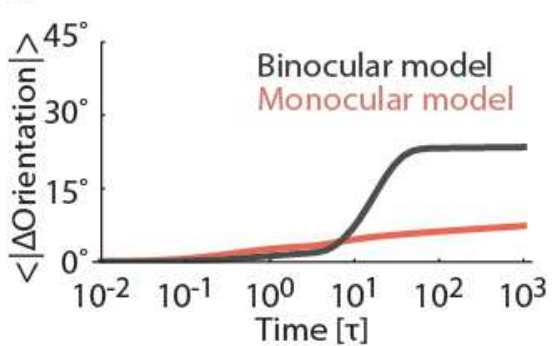

Figure 3.4.1: Results of the binocular model including intra-cortical interactions. a) and b) Plot of the selectivity and the orientation preference for both eyes of one example neuron for $r=0.1, \sigma=1, S=0.25, W=0.75$ and $\gamma=0.7 r$. With that choice of eye-coupling parameters, the neuron is binocular with matching orientation preference, and the matching occurs after the emergence of orientation selectivity. c) Orientation offset between the tuning of each eye in the population. The value only decreases after the development of selectivity. d) Spatial layout of binocular orientations. The tuning is encoded with colors, where the hue is the preferred orientation and the brightness the selectivity. The orientations are matched after the onset of selectivity and the disordered state is maintained. f) Mean absolute change of orientation of the network for the binocular and monocular model. In the binocular case the neurons have a higher change of their preferred orientation since the tuning for each eye has to be matched.

It determines the size and strength of excitatory synapses by organizing glutamate receptors and its associated signaling proteins. Between many other functions, PSD-95 plays an important role in synaptic plasticity. For example, it promotes spine formation and growth through interactions with synaptic RAS GTPase-activating protein and promotes cell adhesion and development through interactions with neuroligin. PSD-95 clusters the N-Methyl-D-aspartic acid (NMDA) receptor and is responsible for its functional localization. An over-expression of PSD-95 in mice increases the magnitude of 2-amino-3-(3-hydroxy-5-methyl-isoxazol-4-yl)propanoic acid (AMPA) receptor associated excitatory post-synaptic currents (EPSC), mimicking long term potentiation (LTP) by converting silent synapses into functional. PSD-95 knock-out mice, on the other hand, have a reduced AMPA/NMDA EPSC ratio, while the strength of inhibitory post-synaptic currents (IPSC) is maintained. In those mutants the number of silent synapses (i.e. with decreased functional AMPA receptors) is increased and maintained into adulthood [152].

It is shown that in PSD-95 knock-out mice the orientation selectivity of neurons doesn't reach normal levels [153]. Recent findings, on the other hand, show that in PSD-95 knock-out mice juvenile ocular dominance plasticity is maintained into late adulthood [154]. That even with a prolonged critical period the orientation selectivity of the neurons doesn't fully mature is intriguing, specially because it was shown that this period determines the temporal window where the orientation of the left and right eye tunings are matched [127]. 
The interplay between ocular dominance and orientation selectivity plasticity in those knock-out mice is a good experimental set up to test the predictive power of the dynamical matching model. What is the impact of the deletion of PSD-95 in the preferred orientation matching between the eyes?

\subsubsection{Tuning of the model}

To predict the degree of binocular orientation matching in a PSD-95 knock-out, first the model has to be tuned to reproduce the behavior of the dynamics found in the experiments.

- Wild type levels of binocular matching

If the equations are solved without noise, the matching between the orientations will be perfect, independent on the choice of parameters in the valid range. Experimentally there is a distribution of binocular orientation mismatch even in late adulthood [127]. Therefore, noise in the simulation is included, where the noise input to the left eye is correlated to the noise input to the right eye with strength $c$.

$$
\begin{aligned}
& \partial_{t} z_{i, r}=F\left[z_{r}, z_{l}\right]+c \sigma \eta_{1}(t)+\sqrt{1-c} \sigma \eta_{2}(t) \\
& \partial_{t} z_{i, l}=F\left[z_{l}, z_{r}\right]+\sqrt{1-c} \sigma \eta_{1}(t)+c \sigma \eta_{2}(t)
\end{aligned}
$$

where $\eta_{1}$ and $\eta_{2}$ are Gaussian white noise with zero mean and noise intensity $\sigma$. A choice of parameters where the final distribution of orientation offset matches the experimental findings is

$$
r=0.1 \quad c=0.1 \quad \sigma=0.510^{-3} \quad \gamma=0.3 r
$$

- Changes in the degree of plasticity in the critical period

In the model the dynamics are time independent. To include temporal changes in the speed of the dynamics of each tuning curve, the time dependency is included in the time-constant $\tau$

$$
\begin{aligned}
\partial_{t} z_{i, r} & =\frac{1}{\tau(t)} F\left[z_{r}, z_{l}\right] \\
\partial_{t} z_{i, l} & =\frac{1}{\tau(t)} F\left[z_{l}, z_{r}\right]
\end{aligned}
$$

Having a time dependent time-constant is inconvenient for the injection of noise in the equation. Without further modification, as the time step is varied the noise statistics remain constant and the diffusion coefficient doesn't reflect the speed of the dynamics. A simple solution for this is to solve the equation with constant time step and link the time in the simulation $t$ in units of $\frac{1}{r}$ with the real time $T$ in days using

$$
t=\int_{0}^{t} \frac{1}{\tau(T)} d T
$$


The smaller the time constant, the longer the equations have to be simulated to represent the same time $T$. The inverse of the time-constant represents therefore the level of plasticity.

The variations in plasticity found in the critical period [17] is modeled as the difference of two exponential functions

$$
\tau^{-1}(T)=B+A\left(e^{-\frac{T}{F}}-e^{-\frac{T}{R}}\right)
$$

where $B$ is the basal plasticity level, $R$ the raise of plasticity and $F$ the fall. The parameter $A$ normalizes the level of plasticity to reach a maximum of 1

$$
A=\frac{1-B}{\left(\frac{R}{F}\right)^{\frac{R}{F-R}}-\left(\frac{R}{F}\right)^{\frac{F}{F-R}}}
$$

For the wild type the parameters chosen are

$$
R=40 \quad F=70 \quad B=0.25
$$

The resulting curve is plotted in green in figure 3.5.1a). The time $T$ is discretized such that equation 3.5.1 becomes a cumulative sum, and the size of the time bins $\Delta T$ is tuned such that the dynamics of the matching parallels the time course in the experiments. The value used is $\Delta T=0.7$. To emulate the prolonged critical period for the PSD-95 knock-out, the parameters of the plasticity are changed to

$$
R=15 \quad F=1000 \quad B=0.25
$$

The resulting curve is platted in red in figure 3.5.1a).

- Decrease in the orientation selectivity in PSD-95 knock-out

The expression for the selectivity of neurons in the $\gamma \neq 0$ model is obtained from equation 3.2.2 $|A|^{2}=\frac{r}{1-4 \gamma}$. The 1 in this equation comes from the cubic term $z_{i, r}\left|z_{i, r}\right|^{2}$ in the dynamical equation. By scaling this term with the parameter $q$, the amplitude becomes $|A|^{2}=\frac{r}{q-4 \gamma}$. An increase in $q$ leads to a decrease in the final selectivity of the neurons. For the wild-type simulations the value used is $q=1$ and for the knock out $q=2$.

\subsubsection{Numerical results}

A two dimensional system of $128 \times 128$ neurons in a box of size $L=2 \pi \sigma$ with periodic boundary conditions was simulated using the modifications described and with lateral interactions given by $S=0.3, W=0.7$ and $g=0$. The initial conditions were neurons with random orientation preference and low selectivity. The equation was solved using a stochastic Runge-Kutta method [155, 156]. The results of the simulation are plotted in figure 3.5.1b) to f). Figure b) and c) show the development of the left and right eye tuning for 2 neurons (light and dark colors) in the wild type (green) and knockout (red) simulations. Straight lines represent the left eye tuning, dotted lines the right eye. In both 
a

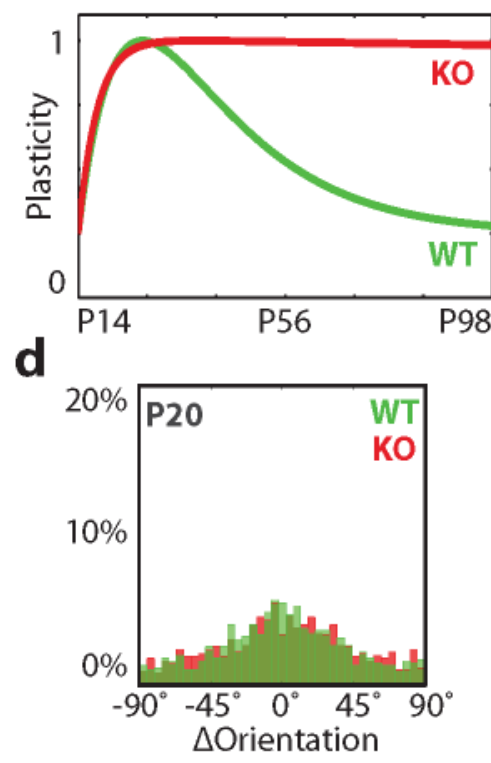

b
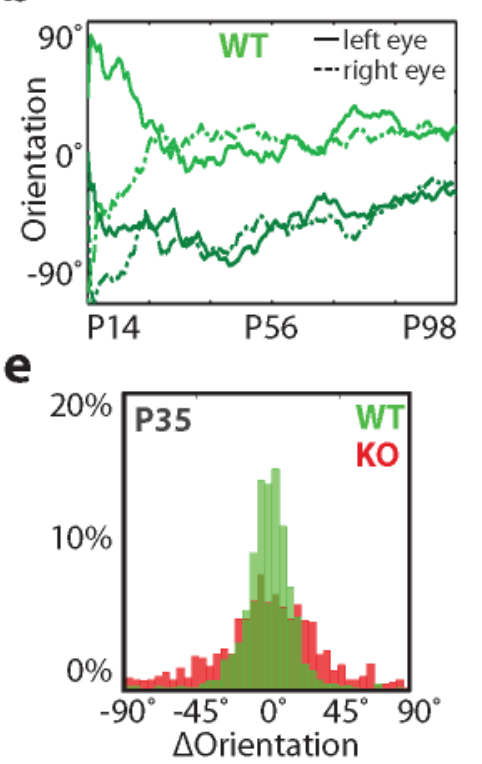

C
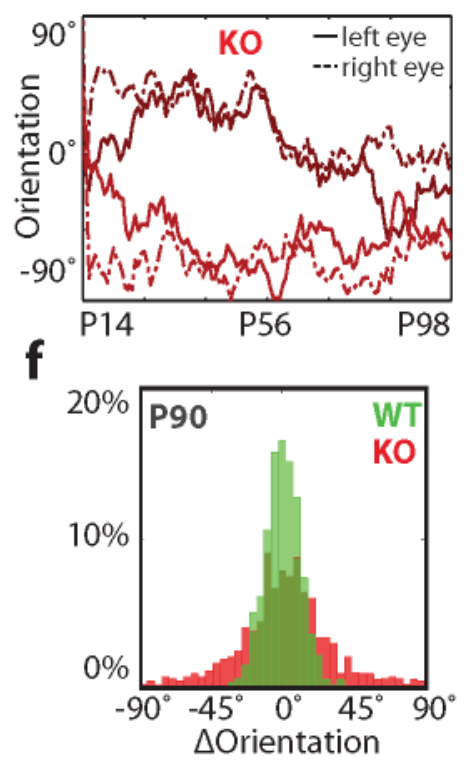

Figure 3.5.1: Predictions of the model in a PSD-95 knock-out experiment. a) Time course of the plasticity used in wild type (green) and knock-out (red) simulations. In PSD-95 high levels of plasticity are maintained into adulthood [154]. b)and c) Development of the orientation preference of the left and right eye tuning for two example neurons in the simulations (dark and light color). Although the orientation offset is decreased, the level of matching and the preferred orientation fluctuates. d) to f) Histogram of the orientation offset for different time points of the simulation. d) At P20 the neurons are orientation selective (not shown) but the level of matching is low. e) At P35 the wild type and knock-out have reached the maximum degree of matching given by the noise levels, where the offset has a wider distribution in the knock-out. f) Even with a prolonged period of high plasticity the levels of matching in the knock out are not increased.

cases the preferred orientations are brought together, but the level of matching and the preferred orientation fluctuates in time. This fluctuation is larger in the knock-out example. In figures d) to f) a histogram of the orientation offset between the eyes at different time points of the simulation is plotted. The wild type is plotted in green and the knock-out in red. At P20, where selectivity already developed (not shown), the offset between the neurons is large in both simulations. At P35 the matching between the orientation increases, but the spread of offsets is higher for the knock-out. This difference is maintained into adulthood in P90, even if the levels of plasticity in the knock-out simulations was increased in this period.

\subsubsection{Discussion}

The model predicts that in PSD-95 knock-out experiments the level of matching between the neurons will decrease. That this is the result of the simulation is no surprise. In the model, the mechanism that matches the orientation after the emergence of selectivity is non-linear, and therefore it is scaled by the selectivity of the neurons. This means that a reduced selectivity decreases the strength of the matching dynamics. With equal amount of noise, an impairment of the selectivity will therefore represent a higher susceptibility to the fluctuations, decreasing the level of orientation matching.

There can be different interpretations if the knock-out experiments are performed and the results 
agree with the prediction of the model. Many interpretations would include a fundamental role of PSD-95 in the biological mechanisms responsible for the binocular orientation matching. This model shows that if PSD-95 affects the selectivity of the neurons, the degree of matching between the eyes will also be affected, even if there is no direct causal link between the two. 


\section{Chapter 4}

\section{Functional implications of the interspersed layout}

In the previous part the necessary conditions for the development of interspersed layouts of orientation preference were derived and successfully tested in a biologically inspired model of oriented receptive field formation. Analytically it was concluded that, with strong local inhibition, both the unselective state and ordered patterns are unstable solutions, making a disordered layout the only possible outcome in development. In this part of the thesis the resulting pattern will be analyzed numerically. The following questions will be answered:

- How are disordered patterns dynamically formed?

- What are the dynamical implications for a neuron embedded in an disordered network?

- Are all disordered layouts equivalent? Is there a spatial structure in the disorder?

- What is the functional benefit of having disordered layouts in the visual cortex?

This part of the thesis is split in two sections. In the first section the dynamical implications of the interspersed layout are explained. It is shown that the developmental dynamics are similar to a glass system, where frustration results in a power law decrease of changes in orientation and energy with time (aging). It is also shown that the high number of disordered solutions has a strong dynamical implication in the susceptibility of neurons to noise, where even small fluctuations will have a strong effect on the stability of the preferred orientation of neurons. In the second section the spatial characteristics of the interspersed pattern are examined in detail. It is shown that a negative correlation in the tuning of the neurons develops, where neurons will tend to have an orientation preference as different as possible from its nearest neighbors. This correlation as a determinant characteristic of interspersed patterns is challenged by the two facts: i) it scales inversely with the number of neurons inside the interaction range, and ii) random patterns with the same negative correlation show a higher amount of change in development as the solutions achieved by the dynamics considered here. Two alternative higher order characterizations are used that are sensitive to different patterns with the same correlation. The fist one is the discrepancy of the layout, measuring the homogeneity of the disorder for any possible pattern interval. The second one has a similar background but a direct 
biological interpretation: the Swindale coverage of the pattern. It measures the homogeneity of the representation by the pattern of any given oriented visual input. Both the discrepancy and coverage are improved in the dynamically generated interspersed patterns. The later result leads in chapter 5 of the thesis to propose an evolutionary transition mechanism between maps and interspersed layouts.

\subsection{Dynamical characterization}

From the analysis of the dynamical equations one can determine the stability of stationary solutions by calculating the growth rate of a perturbation over them. But how the system develops towards that solution is a non-equilibrium physics problem that can't be solved with the mathematical tools used in the previous part of the thesis. Instead, simulations of a developing pattern are implemented, keeping track of the history of each neuron. Having this information one can calculate how much in average each neuron changes its orientation over time and how the energy of the pattern is decreased.

Many of the simulations in this section were performed in a one dimensional system of 1024 neurons without orientation selective interactions following the numerical methods described in section 2.3.3. Selectivity in the interactions was not included since it was shown in the previous part not to be crucial in the determination of the interspersed patterns. Also recent experimental findings [124] show that the selective interaction between excitatory neurons in mouse visual cortex emerges only later in development after visual experience. The results presented here are not strongly modified when orientation selective interactions are used. Using this reduction, the dynamics in equation 2.1.4 simplifies to

$$
\frac{\partial z_{i}}{\partial t}=\tilde{r} z_{i}+\frac{1}{N} \sum_{j} K\left(x_{i}-x_{j}\right) z_{j}-z_{i}\left|z_{i}\right|^{2}
$$

The energy of a layout $z$ in the system can be calculated using $\frac{\partial E}{\partial \bar{z}}=-\frac{\partial z}{\partial t}$, obtaining

$$
E=-r \sum_{i}\left|z_{i}\right|^{2}-\frac{1}{N} \sum_{i, j} \bar{z}_{i} K\left(x_{i}-x_{j}\right) z_{j}+\sum_{i}\left|z_{i}\right|^{4}
$$

A one dimensional system was chosen because of the possibility to detect symmetrically equivalent patterns. Two patterns are equivalent if a combination of a rotation $R_{\beta}$, a translation $T_{y}$ and phase shift $e^{i \varphi}$ makes both patterns equal. If how much a pattern changes in time is measured, a development towards an equivalent pattern should be regarded as no change. The optimal phase shift $\varphi$ that minimizes the angular difference between the neurons in a pattern $z$ and a pattern $y$ is determined by minimizing the following expression:

$$
\sum_{i}\left|z_{i}-y_{i} e^{i \varphi}\right|^{2}=\sum_{i}\left|z_{i}\right|^{2}+\sum_{i}\left|y_{i}\right|^{2}-\sum_{i} z_{i} \bar{y}_{i} e^{-i \varphi}-\sum_{i} \bar{z}_{i} y_{i} e^{i \varphi}
$$

To minimize the angular difference the last two terms have to be maximized. For this we define

$$
B=\sum_{i} z_{i} \bar{y}_{i}=|B| e^{i \phi}
$$


a

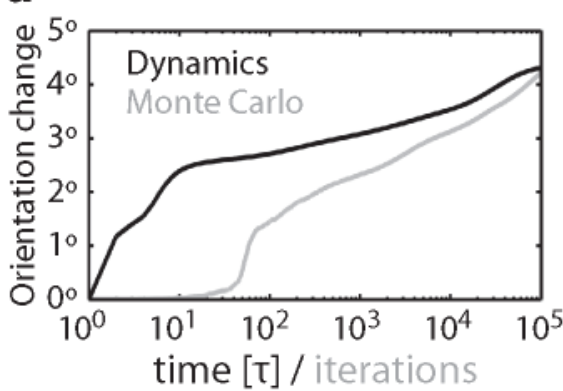

b

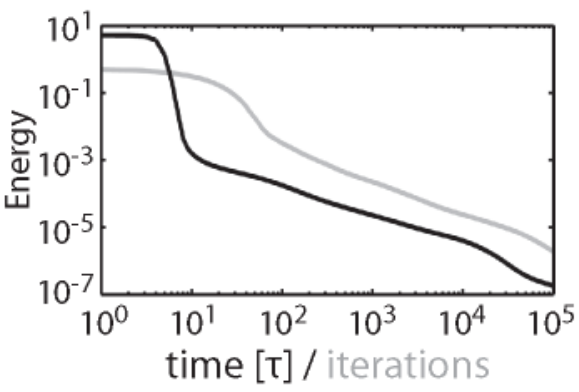

C

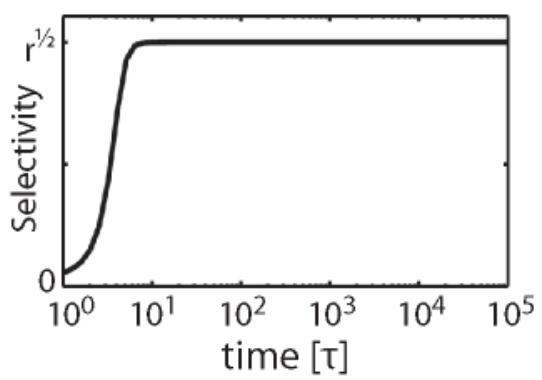

Figure 4.1.1: The development of the interspersed layout is analogous to a glass system where aging in the dynamics takes place. Black curves: Simulation of the system following the dynamics in equation 2.1.4. Gray curves: Monte Carlo approach where the selectivity is fixed and for one neuron at the time the orientation is set that minimizes the energy. a) Mean absolute orientation difference of the system compared to the pattern at $1 \tau$. For Monte Carlo simulations the iterations are discrete and the comparison is done with the orientations of the initial condition. b) Energy decrease in time / Monte Carlo iterations. c) Development of the mean selectivity of the neurons. The one dimensional system is composed of 1024 with interaction parameters $S=\frac{1}{4}, W=\frac{3}{4}$ and $g=0$.

and insert in the last two terms, obtaining

$$
\sum_{i} z_{i} \bar{y}_{i} e^{-i \varphi}+\sum_{i} \bar{z}_{i} y_{i} e^{i \varphi}=B e^{-i \varphi}+\bar{B} e^{i \varphi}=|B|\left(e^{-i(\varphi-\phi)}+e^{i(\varphi-\phi)}\right)=2|B| \cos (\varphi-\phi)
$$

The two terms are maximized with the phase shift

$$
\varphi=\phi=\arg \left(\sum_{i} z_{i} \bar{y}_{i}\right)
$$

In a one dimensional system rotations do not exist and all possible translations with their corresponding optimal orientation shift can be tested simultaneously using toeplitz matrices, making it possible to find the least difference between two patterns very efficiently. Once again, running the simulations in a two dimensional system doesn't modify the results.

\subsubsection{Glassy dynamics}

Choosing the model parameters in the interspersed phase, $S=\frac{1}{4}, W=\frac{3}{4}$ and $g=0$, a network of neurons with initially random orientations and low selectivity was simulated for $t=10^{5} \tau$. At the end of the simulation the pattern is still disorganized and visually very similar to the initial condition. The pattern at each measured point in the development was translated and phase shifted to minimize the difference in orientation compared to the initial condition.

In figure 4.1.1a) the mean orientation change of the pattern for every time-point in development compared to the time-point where selectivity emerges $(t=1 \tau)$ is plotted in black. With $t<1 \tau$ very small absolute changes in $z_{i}$ can cause a large increase in $\arg \left(z_{i}\right)$, so they are not plotted for clarity. The magnitude of the change until $t=1 \tau$ is about $5^{\circ}$. As comparison, the black curve in figure 4.1.1b) shows the decrease in the energy of the pattern in time. Both curves present two different regimes in the tuning curve dynamics of the neurons: After $t>10 \tau$ neurons change their orientation 
with a lower slope than initially in development and the slope of the energy reduction is also highly decreased. This transition can be explained looking at the plot of the average orientation selectivity of the neurons over time in figure 4.1.1c). Although selectivity emerges at $t=1 \tau$, it is not fully developed until $t=10 \tau$, where it saturates at the value $A=\sqrt{r}$. Therefore, the first dynamical regime belongs to the development of the selectivity, where there is a large decrease in the energy as the first and last terms in equation 4.1.1 cancel each other. This corresponds to a higher change in the orientation of the neurons, representing a steeper slope plus the amount of change accumulated before $1 \tau$ that is not plotted. The second dynamical regime belongs to a decrease of the energy only by optimizing the arrangement of orientations in the system. With the saturated selectivity $|A|^{2}=\sqrt{r}$ the energy term that remains belongs only to the interaction between the orientations and can be written as

$$
E^{\text {int }}=-\frac{r}{N} \sum_{i, j} K\left(x_{i}-x_{j}\right) e^{i 2\left(\theta_{j}-\theta_{i}\right)}
$$

Since the interaction $K\left(x_{i}-x_{j}\right)$ needed to develop interspersed patterns the inhibition is locally strong, to further decrease the energy the term $\frac{1}{N} \sum_{i, j} e^{2 i\left(\Delta \theta_{i, j}\right)}$ has to be minimized. This means that every neuron $i$ has to have the neurons $j$ in its interaction range as dissimilar as possible to maximize $\Delta \theta_{i, j}$. But as all neurons try to do this simultaneously, the change in orientation in one neuron is frustrated by the change of the other neurons and the system gets trapped in a disordered state, making every successful movement very slow. This phenomenon is well studied in spin glasses. They are systems of magnetic spins with randomly assigned ferromagnetic and anti-ferromagnetic bonds. The frustrated interactions between the spins in the system lead to a frustration of mutual changes in the spin, leading to a large amount of meta-stable states. Spin glasses exhibit aging in the dynamics, meaning that the time for the system to move from one meta-stable state to another depends on the age of the glass. This aging effect on the timescale of the dynamics is also presented in the simulations with strong inhibition, showing in plots a) and b) as a power law in both orientation change and energy decrease.

To further investigate the role of multiple meta-stable solutions in the generation of interspersed patterns, the achieved dynamics are compared with an algorithm in which for every iteration the shift in the orientation of a single neuron is performed that minimizes the total energy of the pattern. The algorithm is similar to the Metropolis Monte-Carlo scheme [157], where the picked neuron and its assigned shift are randomly chosen and the probability of accepting the shift is proportional to the change in energy of the system $p($ accept $) \propto \max (1 \backslash \exp (-\Delta E))$. The used algorithm differs from the standard scheme in three ways:

- The optimal shift for every neuron is analytically calculated.

- The shift that minimizes the total energy is chosen.

- All iterations are accepted.

Keeping the differences in mind the used algorithm will still be called Monte Carlo. In the algorithm the same random orientations for the initial conditions are chosen, but the selectivity of the pattern is set to $|A|^{2}=\sqrt{r}$ from the beginning and only the orientations are modified. The optimal shift in 
orientation for every neuron can be calculated by introducing a phase shift $e^{i \varphi}$ in one neuron $z_{k}$ and minimizing the interaction term of the energy

$$
\begin{aligned}
E^{i n t} & =-\frac{1}{N} \sum_{i, j} \bar{z}_{i} K\left(x_{i}-x_{j}\right) z_{j} \\
& =-\bar{z}_{k} e^{-i \varphi} \frac{1}{N}\left(\sum_{j \neq k} K\left(x_{k}-x_{j}\right) z_{j}+K(0) z_{k} e^{i \varphi}\right)-\sum_{i \neq k} \bar{z}_{i} \frac{1}{N}\left(\sum_{j \neq k} K\left(x_{i}-x_{j}\right) z_{j}+K\left(x_{i}-x_{k}\right) z_{k} e^{i \varphi}\right)
\end{aligned}
$$

Separating the values of the interaction energy that depend on $\varphi$ leads to

$$
E_{i n t}(\varphi)=-\frac{1}{N} \sum_{j \neq k} \bar{z}_{k} K\left(x_{j}-x_{k}\right) z_{j} e^{-i \varphi}-\frac{1}{N} \sum_{j \neq k} z_{k} K\left(x_{j}-x_{k}\right) \bar{z}_{j} e^{i \varphi}
$$

Defining

$$
B=\frac{1}{N} \sum_{j \neq k} z_{k} K\left(x_{j}-x_{k}\right) \bar{z}_{j}=|B| e^{i \phi}
$$

and inserting in equation 4.1.2

$$
E_{\text {int }}(\varphi)=-\bar{B} e^{-i \varphi}-B e^{i \varphi}==-|B|\left(e^{i(\varphi-\phi)}+e^{-i(\varphi-\phi)}\right)=-2|B| \cos (\varphi-\phi)
$$

Therefore, the shift in the orientation of neuron $z_{k}$ that minimizes the energy of the pattern is

$$
\varphi=\phi=\arg \left(\frac{1}{N} \sum_{j \neq k} z_{k} K\left(x_{j}-x_{k}\right) \bar{z}_{j}\right)
$$

Using this algorithm with interactions in the map phase leads to periodic patterns after a number of iterations in the order of the number of neurons in the system. The results of applying this algorithm in the interspersed phase are shown in gray in figure 4.1.1a) and b), where the x-axis marks the number of iterations used. The energy in $b$ ) starts lower than in the dynamical simulations because the selectivity of the neurons is set at its saturation value from the beginning. The mean change of orientations and the decrease in energy start with very low values in the logarithmic plot since in every iteration only the orientation of one neuron is shifted. As the scale of the logarithm is increased the change in the values is accelerated, but it also enters a second regime where the number of iterations needed to have an equal amount of change becomes a power law.

Using this algorithm it is shown that even when the neurons are manually moved to their energy minima the amount of change and decrease in energy possible in every iteration slows down. This result is in accordance with the idea that the optimal state of each neuron frustrates the optimal state for the rest of the network.

\subsubsection{Energy landscape}

The developing disordered pattern behaves like a glass in the dynamics exhibiting signs of aging. Similarly to spin glasses, this aging might be caused by the existence of a high number of meta-stable 
a

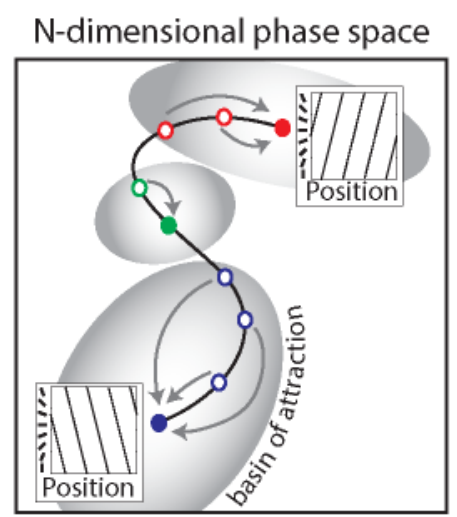

b

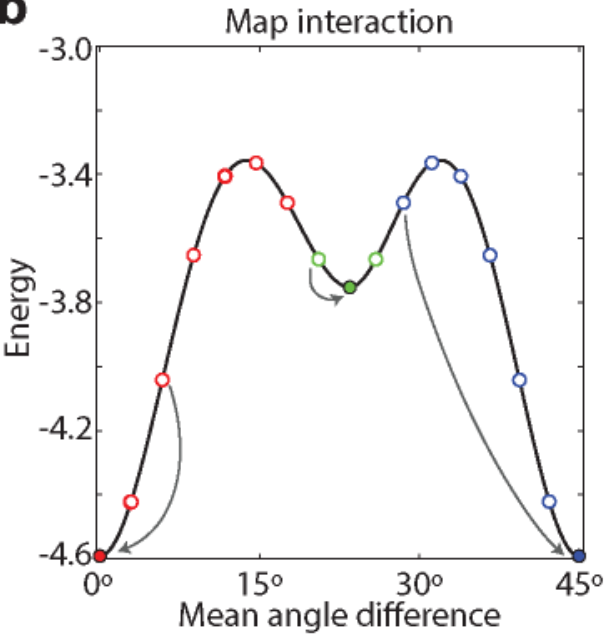

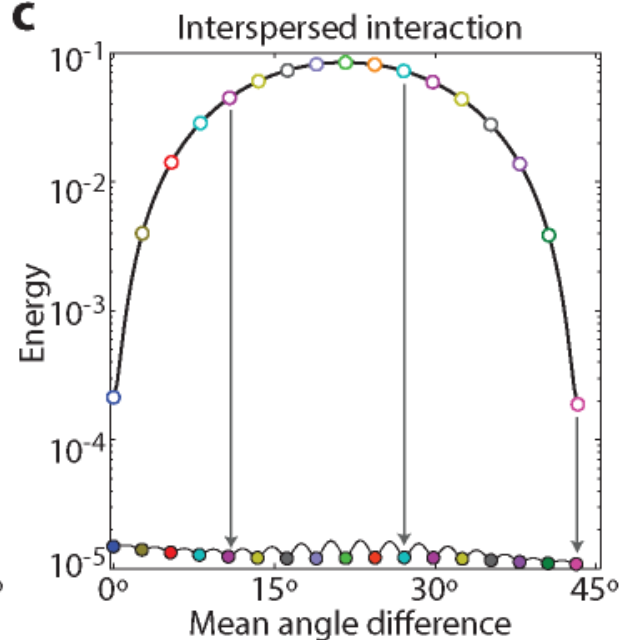

Figure 4.1.2: Multiple meta-stable states in the energy landscape of interspersed solutions. a) Explanation of the approach: A path through the $\mathrm{N}$-dimensional phase space is generated by interpolating between two solutions of a one dimensional system. The path can be characterized by the mean angle difference to the initial pattern. Samples in this path are used as initial condition for a simulation. If the sample sits in the basin of attraction of one solution, it will converge to it during the simulation. Points belonging to the same basin of attraction are color-coded. b) Energy landscape in the generated path (black line) with interactions that generate maps. The energy barriers between the solutions are large, and the number of stable solutions is small. c) Energy landscape in the generated path (upper line) with interactions that generate interspersed layouts. Every initial condition sits in a different basin of attraction. There is a large number of solutions and the energy barriers between them is small.

solutions where the dynamics gets trapped for increasing amounts of time. Here it will shown that for interspersed patterns the number of symmetrically different solutions is very high. This makes the energy barriers between the solutions very shallow, what will have a strong repercussion on how susceptible neurons are to noise.

To show that the number of solutions in disordered patterns is high, a path through phase space will be defined and simulations will be initiated from different sampling points in this path. Points that are inside the influence of the same local energy minima will converge during the simulation. As comparison the same method will be applied to a system with interactions that lead to ordered solutions.

The procedure taken is explained more concretely in figure 4.1.2a). The path in phase space is obtained by interpolating between two different results of the simulations. In the figure this is a pattern with wavenumber $+k$ and with $-k$. The interpolation of the selectivity and the orientation is done separately to keep the selectivity of the neurons high. Distances in the path are measured as the minimum angle difference between the two patterns. This is again the reason to use one dimensional simulations, such that equivalent patterns can be detected. The color code implemented marks with filled circles the results obtained in the simulations, where for every symmetrically nonequivalent solution a different color is used. Colored open circles mark the initial conditions that after $100 \tau$ converge to the patterns marked with the same color.

Figure 4.1.2b) shows the results of this approach in a system with map forming interactions, $S=\frac{1}{4}$, $W=\frac{1}{4}$ and $g=0$. The path in phase space measured in mean angle difference is plotted on the 
x-axis, while the y-axis marks the energy of the interpolated point. To have a smoother path the interpolation was forced to pass through a meta-stable state where the power of the solutions is distributed between the $+k$ and $-k$ modes, where for this choice of parameters $|k|=4$. The figure shows that for every interpolation away from the optimal solution with wavenumber $+k$ or $-k$ there is an increase in the energy of the pattern, but that even with this energy differences initial conditions close to the perfect periodic pattern develop back to the original state. The meta-stable state used as middle point of the interpolations has its own stability range. Longer simulations show that this intermediate state also decays back to a pattern with either $+k$ or $-k$, but for illustration purposes it is kept in the figure. This result shows that in the map phase the amount of solutions is low and the energy barriers between them are large, such that a perturbation to a map solutions will decay in time and the order will be reestablished.

Figure 4.1.2c) shows the same procedure for interactions that form interspersed patterns, $S=\frac{1}{4}$, $W=\frac{3}{4}$ and $g=0$. the extreme points of the interpolations are two disordered solutions obtained from simulations with different random initial conditions. It can be seen that the energy increases between the different interpolated points, but the size of the difference is orders of magnitude smaller than with interpolations in figure b). From the previous section it is known that the system continues developing even for very long time scales, such that the initial points of the interpolation also decrease their energy after $100 \tau$. Independent of this, the result show that none of the points in the interpolation converge, but everyone of them sits in a region attracted by a different local minimum. This behavior does not change for a larger number of samples in the interpolated path or for longer simulations. The results show that in the interspersed case the number of solutions is very large and that the energy barriers between them is low. Although switching between the different solutions will never happen in deterministic development, the presence of noise will make the transition between the different basins very easy because of the low energy barriers and their closeness in phase space.

In the next section these conclusions are tested by including noise in the system and measuring the diffusion coefficient of motion of preferred orientations with time.

\subsubsection{Lability of preferred orientations in the interspersed phase}

The results on the number of solutions in the system depending on the interaction parameters and their impact on the susceptibility to noise are tested in this section. Since the symmetrical equivalence of the patterns is no longer tested, the simulations were performed in a two dimensional network of $128 \times 128$ neurons with selective interactions as described in section 2.1.3. The integration methods was changed to a stochastic Runge-Kutta scheme with fixed time step [155, 156], where Gaussian white noise was injected in every iteration. The time step was chosen as $0.01 \tau$ and the system is simulated for $100 \tau$. The initial conditions of the simulations were the final layout obtained in figure 2.1.1a) and b) in chapter 2. For two dimensional systems the number of solutions for the map case increases, since instead of only two optimal wavenumbers $+k$ and $-k$ the solutions are distributed on the critical circle of radius $\left|\mathbf{k}_{c}\right|$.

The results of the simulations are plotted in figure 4.1.3. Simulations in the map phase are plotted in green and in the interspersed phase in red. Simulations are also performed without interactions to have a comparison of the diffusion caused by noise in a system where neurons only dynamically stabilize their selectivity to $|A|^{2}=r$. Those non-interaction simulations are plotted in gray. 
a
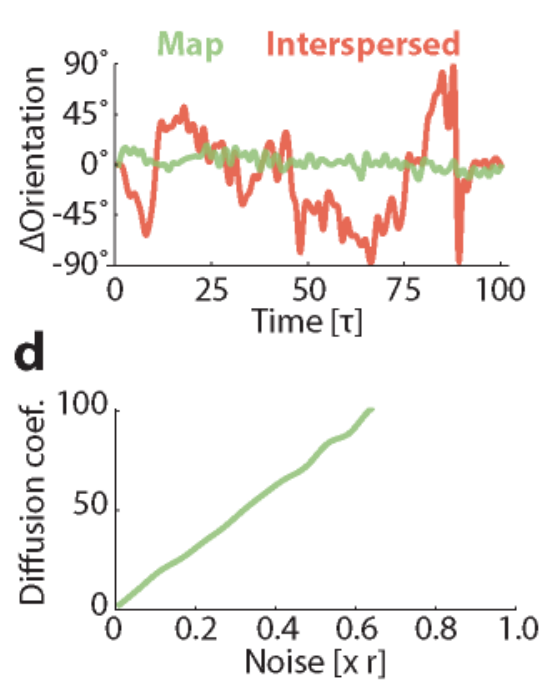

b

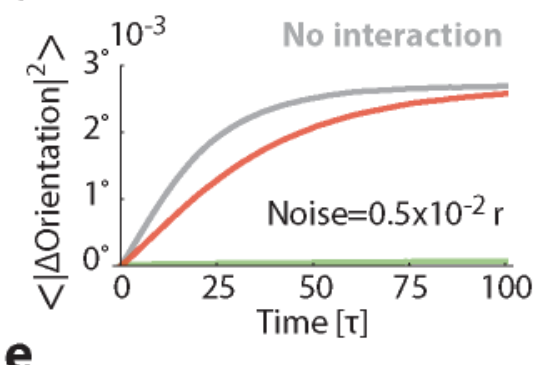

e

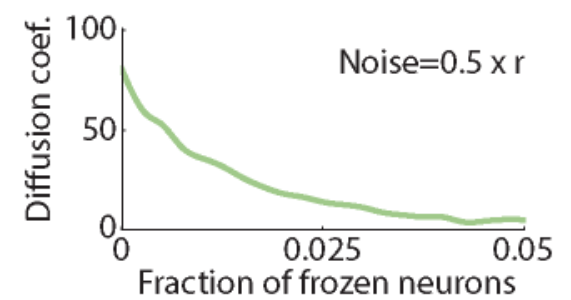

C
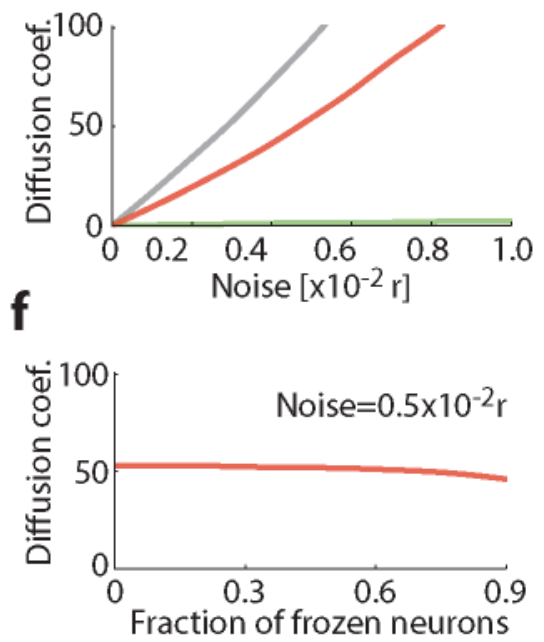

Figure 4.1.3: Neurons in the interspersed pattern are more susceptible to noise. a) Orientation change in time of one neuron in a map (green) and in an interspersed layout (red). b) Mean square orientation difference of the system. In gray the change in a system with no lateral interactions is shown for comparison. Each curve is the mean of 10 simulations. c) Diffusion coefficient of the orientation for each interaction type, obtained by calculating the initial slope in b). d) Diffusion coefficient for the map. Notice that the noise in the x-axis is two orders of magnitude higher than in c). e) and f) Reduction of the diffusion coefficient as the orientation of a fraction of the neurons is frozen, reducing the number of available states in a map (e) and in an interspersed pattern (f). In interspersed patterns the number of available solutions is very high, such that freezing a fraction of neurons doesn't have a strong effect on the diffusion coefficient.

Figure 4.1.3a) shows the orientation over time of one example neuron in each simulation, where the initial orientation is set to zero. The standard deviation of the Gaussian noise is $10^{-2} r$. The represented orientation by the neuron in the map is very stable (as long as the chosen neuron is not close to a pinwheel, where selectivity falls and small changes have a stronger influence on the orientation). In contrast, the orientation of the neuron in the interspersed simulation is changing drastically, passing through many possible orientations in a small amount of time. In figure 4.1.3b) the mean absolute change of orientation of the population in time is plotted, where every curve represents the mean of 10 simulations. With this amount of noise there is a steep increase in the change in the interspersed case, followed by saturation towards $45^{\circ}$ due to the circular constrains of the orientation preference. The neurons are not freely diffusing as if interactions were not present, as can be seen by comparing the results with a non-interacting system in gray. The rise is much steeper and the saturation happens earlier in this case. In contrast, neurons in the map have a very stable representation during the simulation. Figure 4.1.3c) shows the diffusion coefficient $\frac{\mid \Delta \text { Orientation }\left.\right|^{2}}{\tau}$ for different noise levels. It is obtained by calculating the slope of the square increment of the mean change in orientation, where only the initial time steps are used to avoid the influence of saturation. The coefficient increases linearly with the amount of noise. The interspersed coefficient is approximately half of the coefficient for the non-interacting simulations at all noise levels. The diffusion coefficient for the map also increases with higher levels of noise, but the effect is only noticeable if the noise intensity is increased by orders of magnitude. This is shown in figure 4.1.3d). Very high levels of noise in the map overcome the effect of the linear interaction and the characteristic periodicity is lost. 
Taken together the results support the conclusions from the previous section. The neurons in the disordered layouts are more sensitive to noise than in maps by far. The reason is that they have many possible states that can be reached by the perturbation.

The accessibility to some of those states can be blocked by freezing the orientation of a neuron, as all solutions where the neuron has a different orientation as the frozen one will no longer be available. This should reduce the diffusion coefficient in the system. The result of freezing the orientation of a fraction of neurons, while keeping the selectivity dynamical, are shown in figure 4.1.3e) and f) for the map and the interspersed case respectively. In e) the strength of the noise is set at $0.5 \times 10^{-1} r$. Freezing a small fraction of the neurons strongly decreases the available states of the system, making the whole pattern more robust to noise. In contrast in figure f), with the noise level set at $0.5 \times 10^{-2} r$, the freezing of a fraction of neurons also reduces the amount of available states, but they are so numerous that the diffusion coefficient of the remaining neurons is only slightly influenced.

The decrease of the lability of neurons in the interspersed case, even with a large fraction of its orientations frozen, further matches the image of the dynamics of a glass system with many possible states. This last result could be tested experimentally. Some neurons might have a more robust tuning due to mechanisms that are not included here, e.g. stronger connections to the LGN afferents. In the map phase those neurons would work as an anchor point, such that neurons that don't share this property would still be very robust and the map structure would not change. On the other hand, neurons in disordered layouts that are robust won't have a strong influence on the stability of their neighbors, even if they are directly connected. Experimentally this would be measured as a highly varying orientation tuning of some neurons in rodent visual cortex, compared to much more persistent orientation tuning in species with maps. An experimental approach aimed to detect those neurons is presented in part VI of the thesis.

\subsection{Spatial characterization}

In the previous section it was found that in the interspersed case there are many disordered solutions available to the system. But is any random arrangement of orientations a solution? From the analysis of the interaction energy it is known that every neuron will maximize the orientation difference to the neurons it is interaction with. Here the repercussion this has on the spatial structure of the pattern will be analyzed.

\subsubsection{Correlation}

The simplest approach to detect spatial structure in a system is measuring the auto-correlation function. For one dimensional system it is calculated as

$$
C(\Delta x)=\frac{\langle z(x) \bar{z}(x+\Delta x)\rangle_{x}}{\left\langle|z(x)|^{2}\right\rangle_{x}}
$$

For two dimensional systems the radial correlation has the form 
a

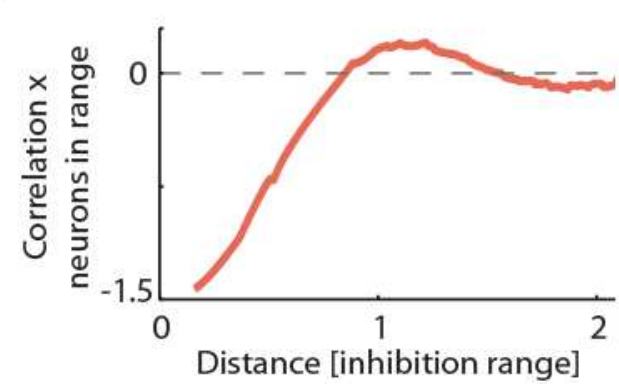

b

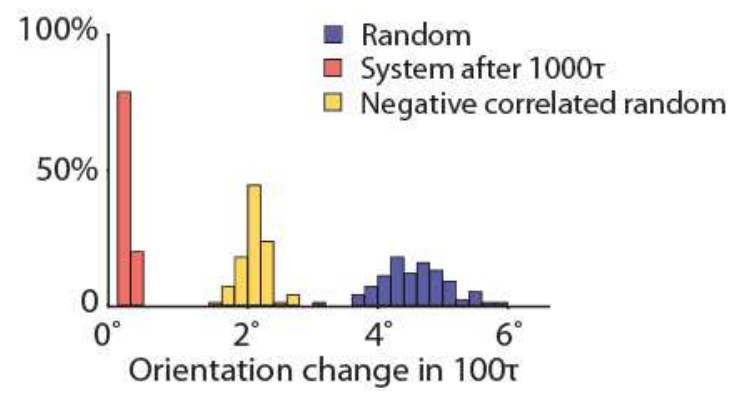

Figure 4.2.1: The structure of the interspersed layout is not random. a) Correlation function between the neurons. Neurons are negatively correlated for short distances. Independent of the choice of parameters, the strength of the correlation is scaled by the number of neurons inside the range of inhibition, and the extend of the correlation depends on the range of inhibition. b) Histogram of changes in orientation for $100 \tau$ for random initial conditions in blue, the system after a long simulation in red, and a random pattern with the same correlation function as the previous case in yellow. The correlation function doesn't fully describe the solutions, as random patterns with the same correlation have a higher change in time.

$$
C(r)=\frac{1}{2 \pi} \int d \phi C(\mathbf{r})
$$

with

$$
C(\mathbf{r})=\frac{\langle z(\mathbf{x}) \bar{z}(\mathbf{x}+\mathbf{r})\rangle_{\mathbf{x}}}{\left\langle|z(\mathbf{x})|^{2}\right\rangle_{\mathbf{x}}}
$$

When measuring the correlation in any one dimensional or two dimensional solution a common result is obtained. It is plotted in figure 4.2.1a). There is a negative correlation for close neurons. The range of this correlations depends only on the range of the inhibitory interaction $(1-W) \sigma$, with no influence of the parameters $S$ and $g$. The strength of the negative correlation depends on the discretization of the system, where the value for nearest neighbors is 1.5 divided by the number of neurons inside the $(1-W) \sigma$ range. The fact that the correlation scales with the number of neurons inside this range agrees with the analogy of interspersed pattern with a glass: with increasing interaction range the more neurons interact with each other, the higher the frustration of the dynamics will be. This means that the correlation is usually very low. For example, for a one dimensional system of size $L$ with 1000 neurons where $\frac{(1-W) \sigma}{L}=0.2$, the nearest neighbor correlation is 0.0075 . In the visual cortex the number of neurons interacting with each other is immense, such that if this negative correlation is present it would be experimentally very difficult to detect.

The negative correlation is not the best spatial description of the final state, since random arrangements of orientation with negative correlation are not solutions. This is presented in figure 4.2.1b). There, a two dimensional system of 256x256 neurons with $S=\frac{1}{4}, W=\frac{3}{4}$ and $g=\frac{1}{8}$ was simulated for $1000 \tau$. The histogram shows the percentage of neurons that changed their orientation a certain amount of degrees during the initial $100 \tau$ from the unselective random initial conditions (blue) and the final $100 \tau$ of the simulations (red). A random arrangement of orientations with the same correlation function as the final pattern was generated by randomizing its phases in Fourier space. The generated pattern was used as initial condition for a simulation for $100 \tau$ and the histogram of the changes in orientation obtained is plotted in yellow. The figure shows that the patterns with equal correlation as the solutions obtained with the dynamics are not equally stationary. This means that 
a

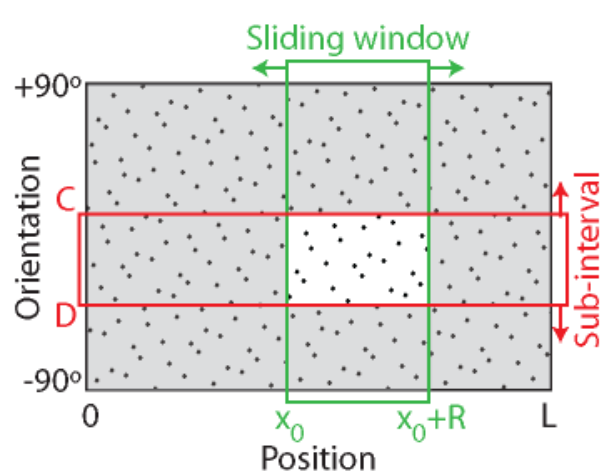

b

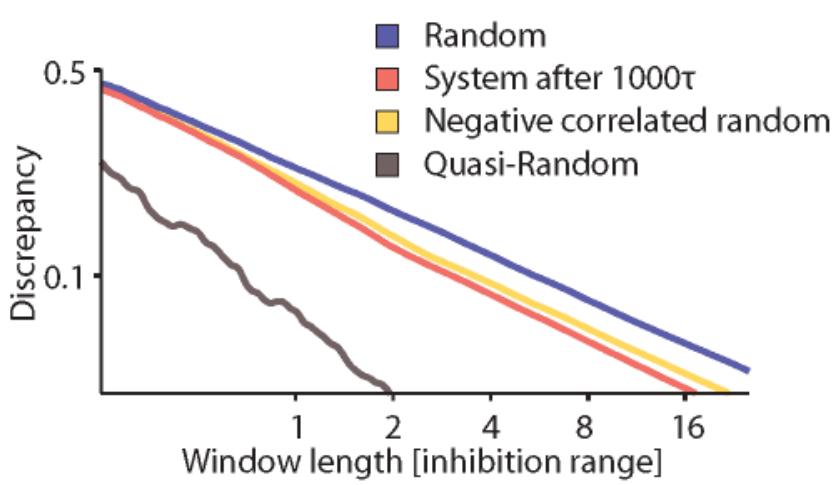

Figure 4.2.2: In interspersed layouts the distribution of orientations is more homogeneous that in random patterns. a) Explanation of the discrepancy measure in one dimensional patterns. For a specific length of the sliding window, orientation sub-intervals of different lengths are tested for homogeneity by comparing the fraction of elements represented in the sub-interval with the relative size of the interval. The maximum difference for all sub-intervals and position of the sliding window is defined as discrepancy. b) Discrepancy of different patterns with increasing window length. Each curve is the mean of 100 simulations. Both axes are logarithmic. The random initial condition is in blue, the solution after a long simulation is in red and a random pattern with the same correlation function as the previous case is in yellow. A computer generated minimum discrepancy arrangement (quasi-random) is plotted in gray for comparison. The discrepancy of the pattern obtained in the dynamics is lower that the discrepancy of random sequences and for phase randomized solutions, but higher than of computer generated quasi-random sequences.

the characteristic structure obtained goes beyond correlation, and that higher order statistics have to be implemented.

\subsubsection{Discrepancy}

The discrepancy measures the homogeneity of a sequence of numbers. For a real sequence $s_{1}, s_{2}, \ldots, s_{N}$ in the interval $[A, B]$, the discrepancy is defined as

$$
D(N)=\sup _{A \leq C \leq D \leq A}\left(\left|\frac{\left\{s_{1}, s_{2}, \ldots s_{N}\right\} \cap[C, D]}{N}-\frac{C-D}{A-B}\right|\right)
$$

where $[C, D]$ is a sub-interval of $[A, B]$. The discrepancy is low if the proportion of elements falling in any sub-interval of the range $[A, B]$ equals the proportion of the length of the sub-interval. In a low discrepancy sequence, any sub-interval has approximately the same number of elements. It is important to note that the order of the elements in the sequence is not important. Random sequences that keep a low discrepancy as more elements are included are called quasi-random and are useful for sampling methods like Monte Carlo integration of functions.

The discrepancy measure was adapted to analyze the homogeneity of the resulting disordered patterns in one dimension. The simulations were performed in one dimension because for comparison a quasi-random pattern like the Halton set can be directly generated using available algorithms. In figure 4.2.2a) the measure is graphically explained, where the example pattern plotted is a quasi-random sequence. The discrepancy of the sequence of preferred orientations of the pattern $\left\{\theta_{1}, \theta_{2}, \ldots \theta_{N}\right\}$ in the interval $[0, \pi]$ was calculated. The interval is periodic, such that constrains on the sub-intervals $[C, D]$ are relaxed to $|\overline{C D}| \leq \pi$. In the previous section it was found that the negative correlation is 
local, so the discrepancy should be more distinguishable from a random sequence for small regions of the pattern where it is not overshadowed by the increasing number of samples. Therefore, the discrepancy was measured for neurons in a sliding window of increasing size $R\left[x_{0}, x_{0}+R\right]$, where the periodic boundary conditions were taken into account in the sliding of the window by bounding its initial point as $\left|\overline{x_{0}, x_{0}+R}\right| \leq L$, where $L$ is the system size. The maximum discrepancy for each window size was used. With these changes, the definition of the discrepancy of a pattern of neurons with orientation $\left\{\theta_{1}, \theta_{2}, \ldots \theta_{N}\right\}$ and position $\left\{x_{1}, x_{2}, \ldots, x_{N}\right\}$ in a window of size $R$ is

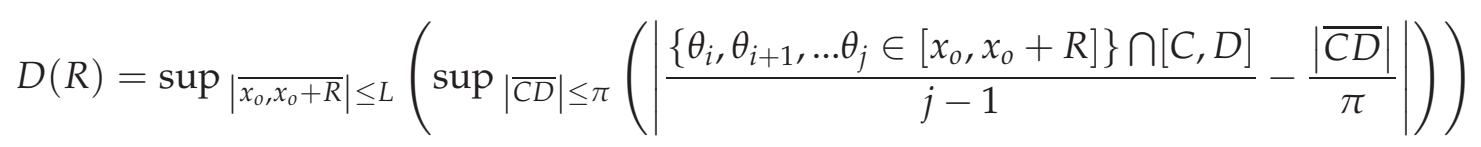

The mean discrepancy as a function of window size of 100 patterns of 1024 neurons and interaction parameters $S=\frac{1}{4}, W=\frac{3}{4}$ and $g=\frac{1}{8}$ is shown in figure 4.2.2b). The random initial condition is plotted in blue, the pattern after $1000 \tau$ in red and a random pattern with equal correlation function as the solution after $1000 \tau$ in yellow. The discrepancy of a quasi-random sequence is plotted in gray for comparison. Both axes are logarithmic, such that straight lines correspond to power exponents.

The results show that the discrepancy of the solution is smaller than the discrepancy of the random pattern for any window size. The discrepancy is also sensitive to patterns with the same correlation, as the values increase in those arrangements. The discrepancy of a quasi-random solution is not achieved, showing that discrepancy is not fully optimized during the dynamics. The discrepancy of the final pattern has a change of slope when the size of the window is approximately twice the range of inhibition. Before that point the slope of the curve is -0.60 and after -0.53 . The slope for a quasirandom sequence is -0.87 and for a random pattern -0.49 . For small pattern lengths, the discrepancy behaves more similar to homogeneously distributed sequences, and as more neurons are taken into account, the behavior is more similar to random sequences. This again shows a spatial regime where the homogeneity of the pattern is increased, which is a result of the interaction range between the neurons. This effect is caused by the correlation structure in the pattern, as phase randomize solutions also present a change of slope. It is important to keep in mind that the change in slope is only apparent when the discrepancy of many patterns is averaged. Here it is shown that the final pattern has an increase homogeneity of represented orientations. In the next section a similar measure will be used with a biological interpretation.

\subsubsection{Coverage}

The homogeneity of the pattern is an interesting structural feature of the interspersed layout, but its relevance in real cortical networks is difficult to interpret. The Swindale coverage of a pattern describes how well the orientation layout can represent any kind of stimulus from a given ensemble [158]. To implement this measure using the result of the simulations further assumptions on the activation function of the neurons have to be used that are not necessary in the discrepancy measure, which depends uniquely on the layout itself. On the other hand, the interpretation of the results is easier.

The concept of the Swindale coverage is represented in figure 4.2.3a). A stimulus in position $r_{x}, r_{y}$ in visual space with orientation $\phi$ is presented to the pattern. The position of the stimulus is translated to a position in cortical space, which as a first approximation assuming retinotopy will also be a 
a

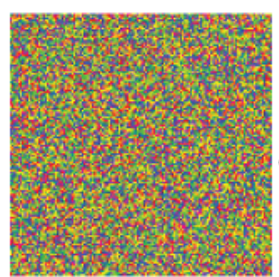

Layout

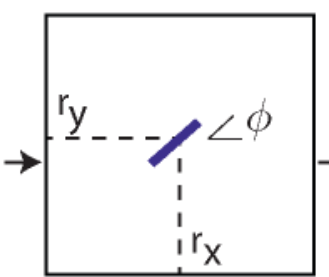

Stimulus

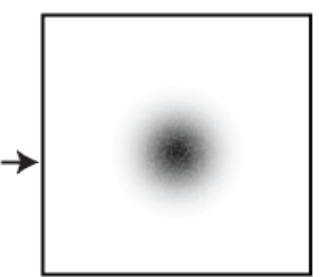

Evoked activity b

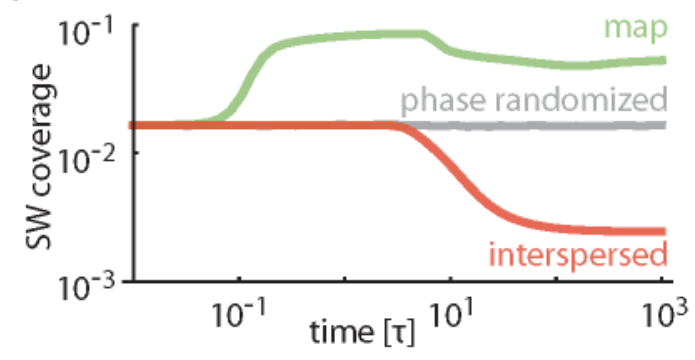

Figure 4.2.3: In interspersed compared to random layouts the stimulus coverage is improved. a) Explanation of the Swindale coverage measure. A stimulus of orientation $\phi$ and position $r_{x}, r_{y}$ is presented to the layout, evoking a response that depends on the tuning of the neurons and their distance to the stimulus. Homogeneity of the total activity across stimulus parameters corresponds to a better coverage of the stimulus ensemble. b) Swindale coverage over time for a map in green and an interspersed layout in red. At every point the interspersed pattern is phase randomized and its coverage plotted in gray. Each curve is the mean of 10 simulations. Although it is not directly optimized in the simulations, the value of the coverage in interspersed patterns decreases monotonically with time and is lower than phase randomized solutions. The simulation parameters are the same as in figure 2.1.1.

scaled version of $r_{x}, r_{y}$. The activity of the neurons elicited by the stimulus depends on how far they are from the center of the stimulation and on how different is the preferred orientation of the neuron compared to the orientation of the stimulus. Depending on the position and orientation of the stimulus, the total activity of the pattern will vary. If the elicited total activity is low for a specific orientation, it means that the pattern does a bad job in representing it. Homogeneity of the total activity across stimulus parameters corresponds to a better coverage of the stimulus ensemble, since the pattern will be optimized to represent all possible stimuli. If $A$ is the total activity elicited by a stimulus $\mathcal{S}$, the Swindale coverage is

$$
C=\frac{\sqrt{\left\langle A^{2}\right\rangle_{\mathcal{S}}}}{\langle A\rangle_{\mathcal{S}}}
$$

Low values of $C$ show that both the mean elicited activity is high, but that its variation in stimulus space is low.

To use this measure of stimulus representation homogeneity, a function that describes the activity of each neuron of the pattern depending on the orientation of stimulus has to be used. For this a Von Misses function is chosen

$$
\Omega(\theta-\phi)=\frac{e^{\kappa \cos (2(\theta-\phi))}}{2 \pi I_{0}(\kappa)}
$$

where $\theta$ is the preferred orientation of the neuron, $\phi$ is the stimulus orientation and $I_{0}(\kappa)$ is the modified Bessel function of first kind. The value of $\kappa$ is $\frac{\pi}{8}$ as in [158]. The modification of the activity depending on the distance to the center of the stimulus is given by the cortical point image $P_{c}(\Delta x, \Delta y)$, defined with a Gaussian function

$$
P_{c}\left(x_{i}-r_{x}, y_{i}-r_{y}\right)=\frac{1}{2 \pi \sigma_{c}^{2}} e^{-\left(\left(x_{i}-r_{x}\right)^{2}+\left(y_{i}-r_{y}\right)^{2}\right) / 2 \sigma_{c}^{2}}
$$


where $x_{i}, y_{i}$ is the location of neuron $z_{i}$ and $r_{x}, r_{y}$ is the position of the center of the stimulus in cortical space, where the magnification factor relating the retinotopical and visual space is set to one. The range of the cortical point image $\sigma_{c}$ is not straight forward to set. What is the typical scale of the interspersed patterns in the simulation? For this the fact is used that the model can also generate ordered map solutions, where the range of the cortical point image can be defined as a function of an hypercolumn size. After choosing a Mexican hat in the linear term to fix the periodicity of the pattern and obtain the column spacing, the Mexican hat is inverted to generate interspersed patterns but the space dependent activation parameters are maintained. The range is thus defined as $\sigma_{c}=0.3 \Lambda$, where $\Lambda$ is the size of the hypercolumn in the map. The value of $\sigma_{c}$ was varied from $0.2-0.5 \Lambda$ without obtaining marked differences. Taken together, the total activity of the map is given by

$$
A\left(\phi, r_{x}, r_{y}\right)=\frac{1}{N} \sum_{i=1}^{N} \Omega\left(\theta_{i}-\phi\right) P_{c}\left(x_{i}-r_{x}, y_{i}-r_{y}\right)
$$

The stimulus ensemble is composed of equally spaced orientations in the interval $0<\phi \leq 180$ centered at every neuron in the cortex. The results of using this activation functions are shown in figure 4.2.3b). The $y$-axis is the Swindale measure of coverage and the $x$-axis marks the development of the value in time as the pattern develops. The initial conditions were neurons with random orientations with low selectivity and the parameters to generate maps and interspersed patterns are the ones described in section 2.1.3. The curves represent the mean result of 100 simulations. The Swindale coverage for the map is plotted in green and of the interspersed pattern in red. At each time point the interspersed pattern is phase randomized, the coverage calculated and plotted in gray.

When an ordered map is formed, the periodicity imposed by the linear term emerges after a very small time scale. The Swindale coverage of these periodic random fields is worse than for random orientations. Intuitively this can be understood as follows: In a map, neurons that represent the same orientation are clustered and the transition of the preferred orientation between the clusters is smooth. This means that neurons preferring two orthogonal orientations are far from each other. If a stimulus is shown to a column preferring an orthogonal orientation the activity will be low, and the activity of a column that is optimized for the presented orientation is damped by the distance. This worsens the coverage of the pattern. When the selectivity of the map is fully developed at $\sim 10 \tau$, the effect of the nonlinear term is strongest, which determines the survival of the modes in the critical circle. As time passes, the pattern reorganizes and unstable modes decay, increasing the homogeneity of the pattern. A more homogenous pattern represents a more homogeneous representation, leading to a decrease in the coverage measure, but the values of the initial conditions are not reached.

As interspersed patterns develop the homogeneity of the layout is increased as neurons mutually repel their orientation preference. This means that neurons representing all possible orientations are very close to each other. If any given stimulus is presented to the pattern, independent of its position, there will always be a neuron close by that is optimized to this orientation, giving a low coverage measure. Similar to the energy of the pattern as a function of time in figure 4.1.1b), this effect is faster while the selectivity is still being developed, since big changes in the orientation can be achieved with small absolute values of $z$. After this point the rearrangement of the orientations continues, but with a smaller slope due to the frustration in the dynamics.

The effect of the correlation on the value of the coverage is much smaller than in the case of the discrepancy, as the coverage of random and negative correlated random orientations is hardly dis- 
a

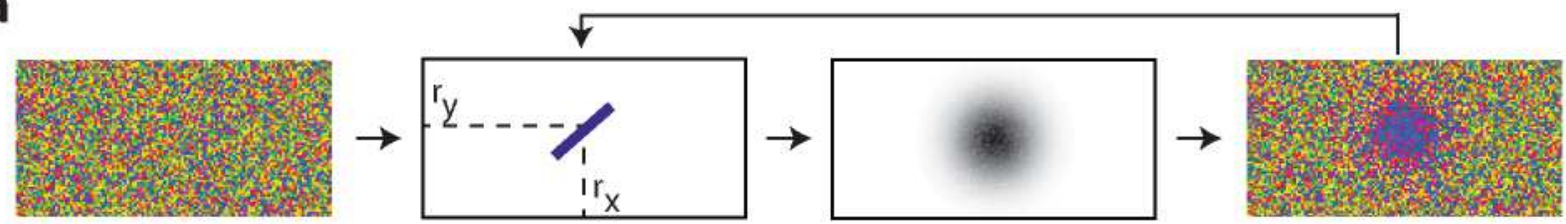

Stimulus

b

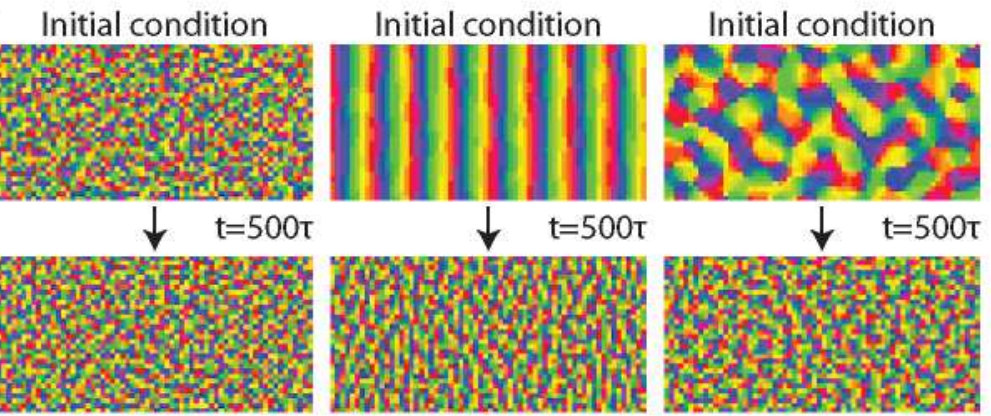

Evoked activity

C

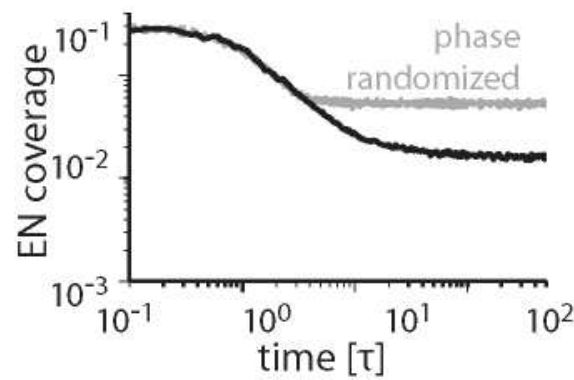

Figure 4.2.4: A model designed to increase the stimulus coverage leads to interspersed patterns [145]. a) Learning dynamics in the model: A stimulus $\mathbf{S}$ described by parameters $s_{z}=\left|s_{z}\right| e^{2 i \phi}$ and $\mathbf{s}_{r}=\left(r_{x}, r_{y}\right)$ is presented to the pattern, evoking activity in the neurons depending on their preferred orientation and the distance to the stimulation site. Lateral interactions are included by normalizing the total activity of the pattern, leading to soft competition between the neurons. Notice that neighboring neurons are co-activated. The activity of each neuron leads to a plastic change in its representation to better match the stimulus parameters. In this case the learning rate is exaggerated. The stimulus presentation is repeated for another set of parameters and the tuning dynamics is described by averaging the changes for a large number of presentations. The energy optimized in the model describes the homogeneity of the evoked activity and is called elastic net coverage. b) Different initial conditions are used and the pattern after $500 \tau$ is plotted. The dynamics actively disorganize the spatial structure of the orientations, leading to interspersed patterns. c) This disorganization is achieved by a reduction of the elastic net coverage. The coverage measure is sensitive to solutions with equal correlation structure, which are plotted in gray. All plots are modified from [145].

tinguishable in the first. It is important to note that the negative correlation is small and that the range of the point spread image is larger than the range of the correlation, making its effect weak. Similar to discrepancy, the effective range of the organization of orientations is larger than the range of inhibition, such that the improvement in coverage goes beyond the correlation range.

Although the coverage is not explicitly optimized in the tuning dynamics, its monotonic decrease suggests a functional consequence of the development of the pattern. A model where the coverage is explicitly optimized was treated by Keil in his $\mathrm{PhD}$ thesis [145]. The approach is based on the elastic net model of cortical map formation [55]. The procedure is explained in figure 4.2.4a). Based on Hebbian learning rules, every represented stimulus by the neurons causes a plastic change in their tuning towards the parameters of the stimulus

$$
\delta z_{i}=\varepsilon\left(s_{z}-z_{i}\right) e\left(\mathbf{x}_{i}, \mathbf{S}, z(\cdot)\right)
$$

The stimulus $\mathbf{S}$ is described by parameters $s_{z}=\left|s_{z}\right| e^{2 i \phi}$ and $\mathbf{s}_{r}=\left(r_{x}, r_{y}\right)$, where $\phi$ denotes the orientation of an activity pattern and $\mathbf{s}_{r}$ its position. $\mathbf{x}_{i}$ is the position of the neuron $i$ and $z_{i}$ its tuning. The parameter $\varepsilon$ scales the strength of the modification caused by one pattern. The activity of the 
neurons is influenced by the similarity of the tuning to the presented pattern $s_{z}-z_{i}$, the distance to the center of the stimulus $\mathbf{s}_{r}-\mathbf{x}_{i}$, and the activity of neighboring neurons. The last one is modeled by soft competition, where the activity of each neuron is normalized by the total activity of the pattern

$$
e\left(\mathbf{x}_{i}, \mathbf{S}, z\right)=\frac{e^{-\left(\left|\mathbf{s}_{r}-\mathbf{x}_{i}\right|^{2}\right) / 2 \sigma^{2}} e^{-\left(\left|s_{z}-z_{i}\right|^{2}\right) / 2 \sigma^{2}}}{\frac{1}{N} \sum_{j}^{N} e^{-\left(\left|\mathbf{s}_{r}-\mathbf{x}_{j}\right|^{2}\right) / 2 \sigma^{2}} e^{-\left(\left|s_{z}-z_{j}\right|^{2}\right) / 2 \sigma^{2}}}
$$

This divisive normalization can be derived from detailed network models with recurrent inhibitory interactions [159]. The influence of a stimulus to a neuron depends on the interaction range $\sigma$, which in the model plays the role of the bifurcation parameter (see [145]). The tuning dynamics can be converted to continuous by averaging a large number of stimulus presentations

$$
\frac{\partial z_{i}}{\partial t}=\left\langle\left[s_{z}-z_{i}\right] e\left(\mathbf{x}_{i}, \mathbf{S}, z\right)\right\rangle_{\mathbf{S}}
$$

The dynamics of the pattern is obtained by $\frac{\partial E}{\partial \bar{z}}=-\frac{\partial z}{\partial t}$ from the energy

$$
E=-\sigma^{2}\left\langle\ln \left(\frac{1}{N} \sum_{j}^{N} e^{-\left(\left|s_{z}-z_{j}\right|^{2}+\left|\mathbf{s}_{r}-\mathbf{x}_{i}\right|^{2}\right) / 2 \sigma^{2}}\right)\right\rangle_{\mathbf{S}}
$$

In this model the energy is equivalent to the Swindale coverage, where the value is minimized when the pattern equally represents the whole stimulus ensemble. Therefore, this dynamics optimizes the coverage of the stimulus space. The model differs from the elastic net by not including an extra continuity term in the energy, which imposes a smooth transition of orientations in the pattern.

As shown in figure 4.2.4b) the pattern that optimizes the coverage obtained in the simulations is disordered. Other ordered arrangements used as initial conditions decay during learning, leading to interspersed layouts. The details of the simulations are given in [145]. That the solution is disordered can be inferred directly from the conclusions of the parameter model in the first part of this thesis, since the model satisfies all the symmetry conditions and the soft competition between the neurons is equivalent to strong local inhibition. The decrease of energy in the model, which is equivalent to the coverage, is plotted in figure 4.2.4c). It is very similar to the decrease in the Swindale coverage for interspersed patterns in figure 4.2.3b), although in the second the coverage is not directly optimized.

Taken together these results show that an improvement in coverage is an essential and robust feature of the dynamical generation of interspersed patterns. Disordered layouts have a benefit compared to maps that all possible stimuli are more homogeneously represented. It is important to note that the coverage of the ordered map will depend on how many columns are spanned by the cortical point image. The more neurons with different orientations are activated by the same stimulus, the more homogenous the activation will be, improving the coverage of the pattern. In the next part of the thesis this effect will be investigated in more depth and its potential role in driving a transition between maps and interspersed patterns will be examined. 


\section{Chapter 5}

\section{Understanding evolutionary transitions between the different layout types}

In the first part of the thesis it was shown that the transition from dynamically generated orientation maps to interspersed patterns can be achieved by increasing the amount of intra-cortical inhibition between the neurons. This result explains how some mammals can build maps and others interspersed architectures using Hebbian rules. It, however, doesn't answer the question why some species evolved to have ordered maps and others not. Are there specific benefits of disordered layouts and ordered maps? The fact that rodents have orientation tuned neurons but no maps shows that the later are not essential for the generation and maintenance of orientation selectivity in the cortex.

In this chapter of the thesis the role of the area size in determining the functional layout of the cortex is investigated. A cost function depending only on structural features of the layout is constructed combining stimulus coverage and wiring cost per neuron. Taking into account the fact that the size of orientation hypercolumns in V1 is approximately constant for maps and brains of all sizes [27], this cost-function predicts a transition from optimal layout as the cortex increases in size. This results because in maps the coverage is largely improved by the addition of columns as the size of V1 is expanded, while adding neurons to the interspersed pattern doesn't have the same impact. On the other hand, the vicinity of a neuron remains unperturbed by the addition of columns or neurons, such that the wiring cost of selective connections between the neurons with similar orientations are lower in maps than in interspersed patterns.

This work was done in collaboration with Wolfgang Keil. He wrote the first version of the code to calculate the wiring cost per neuron following [64] and ran the preliminary simulations.

\subsection{Area size and functional layout}

The concept of using area size as determinant for the functional layout of the primary visual cortex follows directly from looking at the species with and without maps that have been characterized so far, as presented in figure 5.1.1. The plot shows for the different mammalian lineages some examples of the species that have maps in green and the species that have an interspersed pattern in red. 


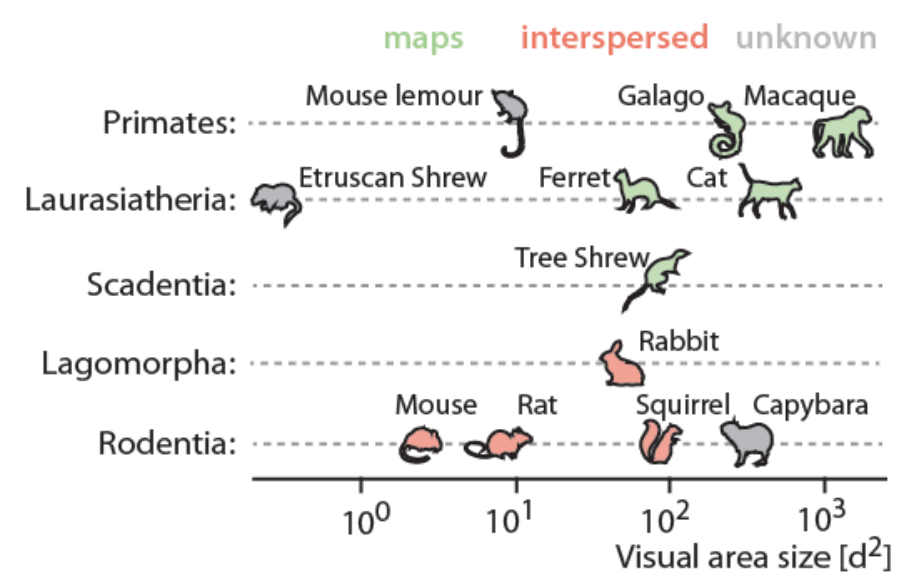

Figure 5.1.1: Area size and functional organization of the cortex in different mammalian lineages. In animals displayed in green ordered orientation maps have been found. In red animals, the pattern of orientation is interspersed. Gray animals haven't been tested. Although there appears to be a clear separation between the lineages and layout organization, this might also be a reflection of the fact that most glires species tested have a smaller visual cortex than the primates and carnivores used. There is a small overlap in cortex size with different layouts, but this can represent a broader transition region between the optimality of the layout types. Data taken from $[98,161-170]$.

Species that haven't been characterized are in gray. The $x$-axis marks the typical size of the visual cortex in the different species. On the one hand the differentiation is clear between mammalian lineages, as the rodents and lagomorphs tested don't have maps and the primates, scadentia and carnivores tested do. On the other hand this result is biased by the size of the cortex of the animals classified. So far, in all the rodents and lagomorphs tested the cortex is smaller or of similar size as the cortex of the tested species with maps. That cortex size needs to be considered as a potentially critical factor is clear when considering the overall course of the mammalian evolution. During the Cretaceous period from 148 to 65 millions of years ago, the common ancestor of all Eutheria weighed between 6 and 245 gram [160]. This means that the size of its cortex was very small. Only after the extinction of dinosaurs in the Cretaceous-Tertiary extinction event 65 millions of years ago the competition for ecological space was presumably reduced and the mammals started to fill and create large animal niches [160], leading to a growth of the cortex independently in the different mammalian lineages [27]. Assuming that due to its size the common ancestor had the functional organization of rodents, at some point it must have been evolutionarily favored in species with large cortices to generate maps similar to the ones found today. Small species like the ones tested maintained the original disordered pattern, while in larger species a change in the interaction between the neurons allowed for the emergence of a self-organized ordered pattern.

To give this hypothesis a precise meaning and to understand conditions under which selection forces might have driven such a transition this chapter will build an optimization theory. A plausible optimization criterion will be identified that is minimal for disordered layouts for small sized brains and for maps of large areas. The aim is not to prove that animals have this criterion optimized, but rather to provide insight to the adaptations and selective forces that might have shaped the evolutionary dynamics [5].

A connection of area size and the functional architecture can already be seen in species with ordered layouts. In species with maps the size of the hypercolumn is approximately preserved in evolution. 
Figure 5.1.2a) shows that although tree shrews have a body weight more than ten times smaller than cats, the typical size of the hypercolumn barely changes [27]. This supports the notion that the cortex grows by adding columns rather than by increasing their size [171]. This also holds during development. As the size of the cortex grows in cats, the size of ocular dominance columns remains constant [172]. This effect has a repercussion on the coverage of the pattern, as explained in figure 5.1.2b). It was previously shown in this thesis that patterns that maximize the coverage to the stimulus parameters are disordered. Coverage is also dynamically improved in those patterns even if it is not explicitly required in the dynamical equation. But the difference in coverage compared to maps depends on the size of the cortex. As the area increases, the number of hypercolumns representing the same region of visual space also rises. With more columns representing the same input the coverage to the stimulus parameters is improved. In interspersed patterns, on the other hand, the homogeneity of represented orientations for any interval of the visual cortex is already increased, such that increasing the number of neurons doesn't have a strong effect on the coverage.

Stimulus coverage favors the formation of interspersed patterns, but its relative advantage decreases with area size. Therefore, a combination of coverage with other optimization criteria that reverse the favoritism will naturally lead to a predicted transition from interspersed to map organization with area size. One such criterion is the wiring cost per neuron that was previously proposed by [64]. The authors analyzed the functional layout that minimizes the total wiring length between the neurons when a specific orientation dependent connectivity function is assumed. If the number of afferents to a neuron has to be equal for every orientation difference, the layout that minimizes the wiring costs is disordered, as in that case all orientations are represented close to each other. On the other hand, if the number of afferents to a neuron needs to be increased for inputs of same orientation tuning, the solution that minimizes wire length are ordered patterns, as neurons with similar properties are close to each other. It was recently shown that in the primary visual cortex of mice neurons with similar orientation tuning have a higher connection probability [115]. Orientation selective interactions are therefore present in both species with and without orientation maps, implying that the wiring costs to maintain this connectivity are higher in interspersed patterns. While for coverage this difference in the cost is reduced with increasing visual cortex area, the wire length per neuron remains constant. This is because the functional composition of the vicinity of a neurons is not influenced by the size of the cortex.

A tradeoff between coverage and wiring costs per neuron leads to a transition in the layout of the primary visual cortex with V1 size. If the cortex is small, the optimal layout are interspersed patterns because the coverage in maps is low and the benefit of lower wiring costs is outweighed. For large cortices, the optimal layout are maps because the wiring costs to maintain orientation selective interactions is low and the difference in coverage is only minimally reduced. In the next section this effect will be studied in detail by constructing and calculating the cost function for both organizations as a function of V1 area sizes.

\subsection{Setting up the calculations}

To check that the effect of the area size on the coverage and wiring cost follows the hypothesis presented, the layouts of different sizes have to be constructed following the rules described.

Ordered maps of increasing size are generated by defining the hypercolumn as $\left(n_{\Lambda}\right)^{2}$ neurons and 
a

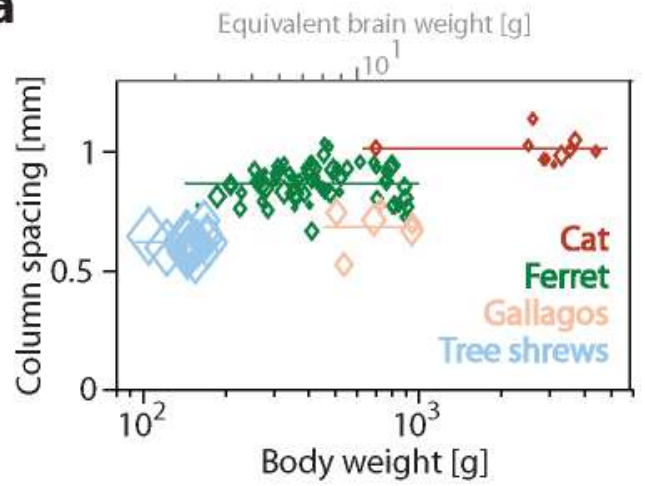

b

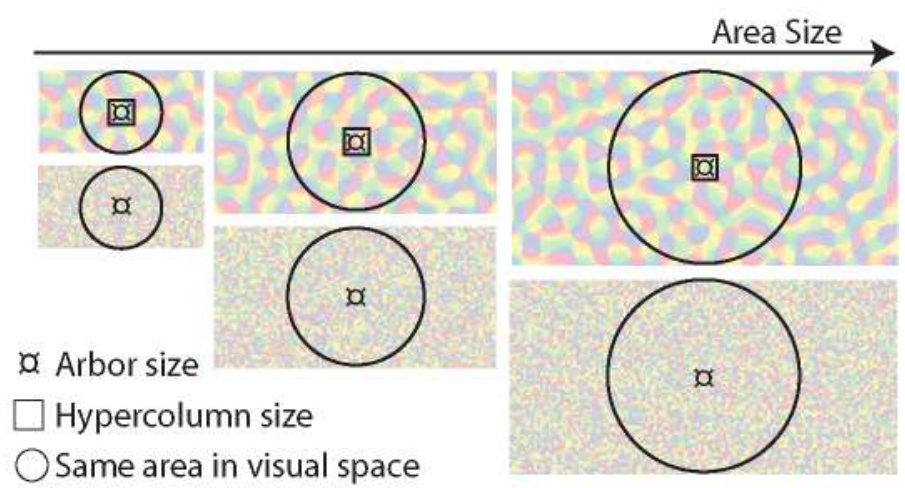

Figure 5.1.2: Functional layout with increasing cortex size. a) Typical hypercolumn size for different animals compared to their body weight. Even with logarithmic increase in the size of the animals, and equivalently their visual cortical area, the size of the hypercolumns changes little. b) With constant hypercolumn size (squares), an increase in the size of the cortex leads to the involvement of more columns with different orientations to represent the same area in visual space (circles). The vicinity of each neuron (asterisks) remains the same independent on the size of the cortex. Figure a) is adapted from [27].

adding discretized number of columns $L$ to the cortex. By setting the hypercolumn size to $1 \mathrm{~mm}, L$ determines the length of the system in $\mathrm{mm}$. For a system of $\left(L n_{\Lambda}\right)^{2}$ neurons, the map is generated in Fourier space by assigning a nonzero value to 20 modes equally distributed in the critical circle of size $L$, as shown in figure 1.3.1a). Reducing the number of modes leads to a more crystalline pattern with lower Swindale coverage values as discussed in figure 4.2.3b). This has as effect the shift of the transition towards ordered maps. As first approximation maps with a high number of modes, and therefore pinwheel density close to $\pi$ are used.

Interspersed patterns are generated by assigning random orientations to the system of $\left(L n_{\Lambda}\right)^{2}$ neurons. Previously in the thesis it was shown that interspersed patterns are not random. The optimized homogeneity of orientations is local and depends on the interaction range between the neurons, which doesn't change with cortex size. Therefore, including the fine structure of the disordered layout only shifts the transition between the layout types towards interspersed patterns, making random orientations a good approximation in this qualitative model.

To measure the Swindale coverage of the layout $C$ the definition in equation 4.2.1 is used. The size of the point spread image is scaled with the cortex size as $\sigma_{c}=p L n_{\Lambda}$, where $p<1$. This is done to increase the number of involved columns in representing a stimulus as the cortex gets larger. The choice of $p$ only determines the relative amount of neurons where activity is evoked by a stimulus, such that its exact value only shifts the transition towards interspersed patterns for smaller choices. The other parameters for the calculation of the Swindale coverage are the same as used in the previous part of the thesis.

To calculate the minimal wiring costs per neuron $W$ the steps described in [64] are followed. First, a discretized connection function between the neurons is imposed

$$
C F(\Delta \theta)=\left[N_{\alpha}\left(b+\frac{e^{\kappa \cos (2 \Delta \theta)}}{e^{\kappa}}\right)\right]
$$

The connection function describes with how many neurons with orientation difference in the interval 
$\Delta \theta$ each neuron has to be connected with. $N_{\alpha}$ is a normalization factor to ensure that each neuron has exactly $\alpha$ afferents and the square brackets represent rounding the result for each bin since only only integer number of connections are possible. The parameter $\kappa$ determines the shape of the interaction function. Smaller $\kappa$ makes the distribution broader, such that the number of imposed connections to neurons for each orientation difference bin $\Delta \theta$ is similar. Larger $\kappa$ makes the distribution more peaked. The constant $b$ is chosen to ensure that every neuron has at least one afferent of every orientation difference bin. For a fixed connectivity function, the minimal wire length to satisfy it is calculated neuron by neuron by binning the orientation difference between its neighbors, looking for the closest $C F(\Delta \theta)$ neurons for each orientation difference bin and summing the distances.

The parameters used in the calculations are $n_{\Lambda}=20, p=0.02, \alpha=\pi\left(\frac{n_{\Lambda}}{4}\right)^{2}, b=0.12$ and $\kappa=3$. The orientation difference in the connectivity function is divided into 15 bins. The area of the system was varied from $10^{1}$ to $10^{3} \mathrm{~d} m^{2}$ The only parameters that are crucial for this approach are the ones defining the connection function. This will be analyzed with more detail later in the text.

\subsection{Results}

For every cortex size and functional architecture 30 patterns were generated and their Swindale coverage and minimal wiring cost per neuron calculated. The results are shown in figure 5.3.1a) and b).

Figure 5.3.1a) shows the Swindale coverage of the interspersed pattern in red and for the map in green as a function of area size. The points are the actual measurements and the line is a power fit passing through them. The variation of the measured values for every area size is small, such that the fit is a good representation of the data. For maps the equation used is $C_{\text {map }}(a)=0.76 a^{-0.36}$ and for interspersed patterns $C_{i s p}(a)=0.27 a^{-0.50}$, where $a$ is the area size. Although for both cases the value of the coverage decreases with increasing size, the difference gets smaller the more columns are involved in the representation. The results thus follow the predictions presented.

Figure 5.3.1b) shows the minimal wiring costs per neuron of the interspersed pattern in red and for the map in green. In the case of the interspersed patterns the variation for the different sizes is small. It is evident that the value is insensitive to a change in cortical area, such that the result can be described with the constant $W_{i s p}(a)=17.58$. In the case of the map there is a high variation in the measure obtained for every area size. Since the pattern is not crystalline, different realizations have pronounced differences in the fine structure of the orientations, leading to the high variation of the wiring costs for every neuron. A possible solution to make the measurements less variable is to decrease the number of modes used to generate the maps or use the median wiring costs per neuron instead of the mean. From the plot it is clear that the mean value of the wiring cost and the size of the fluctuations are independent of the cortex size, such that it can be described by the constant value $W_{\text {map }}(a)=17.01$. The results of the wiring costs show that, as predicted, the cost is larger for interspersed patterns than for maps and constant with respect to cortex size.

Figure 5.3.1c) shows the result of joining both optimization parameters linearly in a combined cost function

$$
F=C+\alpha W
$$


a
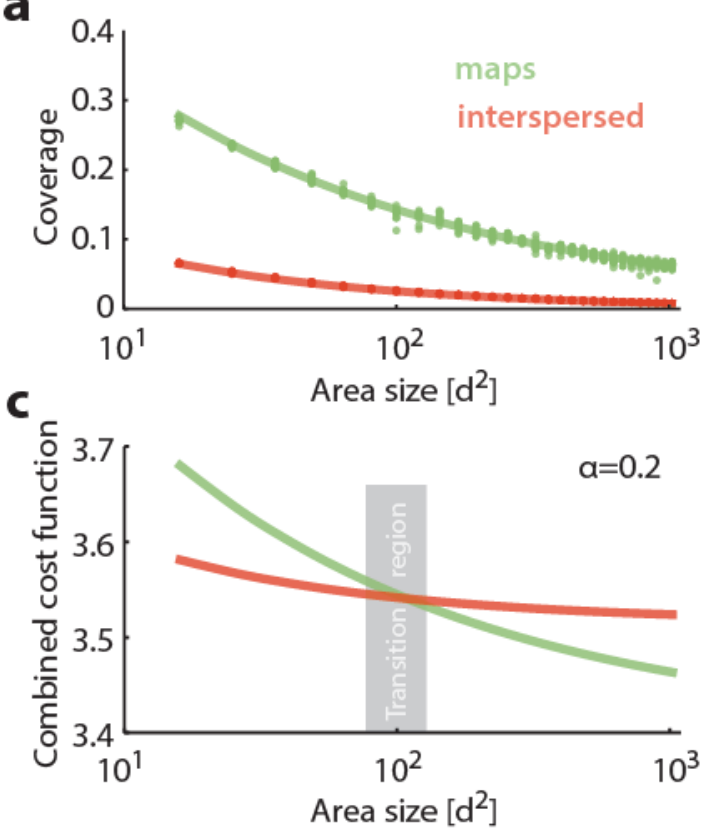

b
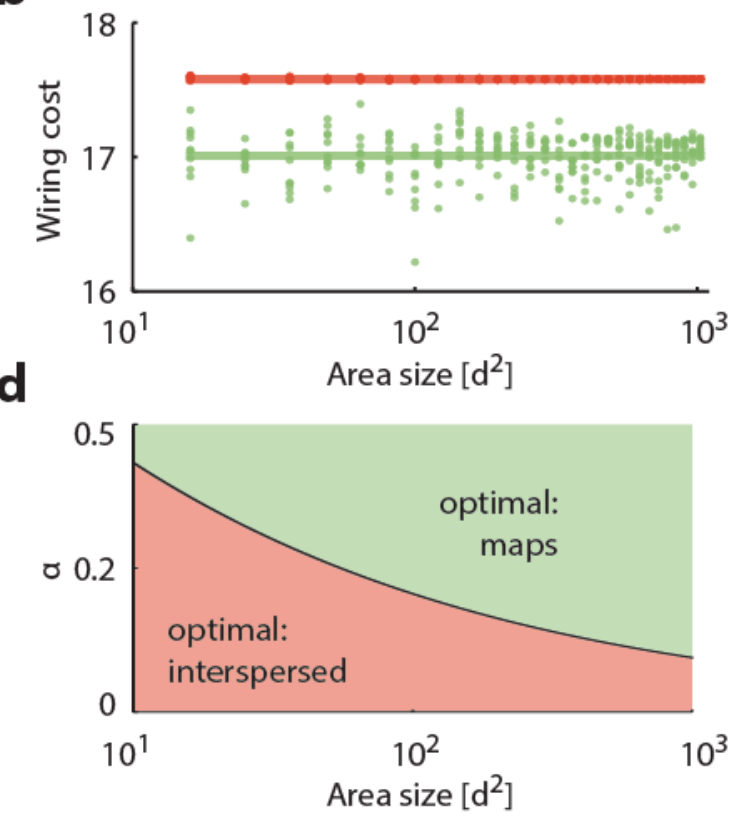

Figure 5.3.1: Swindale coverage and wiring costs for the different layout types of varying size. In all plots the measurements for maps are displayed in green and for interspersed patterns in red. 30 realizations of each pattern type and size are analyzed. a) Swindale coverage of the patterns. The coverage is improved with the addition of neurons responding to the same stimulus for both layouts, but the difference is reduced with increasing size. b) Minimal wiring costs per neuron for the patterns. Although there are big fluctuations for the different realizations, the costs remain constant with increasing cortex size and are always lower for maps than for interspersed patterns. c) Combined cost function where the wiring cost is weighted by the parameter $\alpha$. In the plot the fitted curves for the measurements are used. After $10^{2} \mathrm{dm}$ there is a transition between interspersed patterns being optimal to ordered maps being optimal. d) Optimal layout depending on area size and the parameter $\alpha$. For every choice of parameter $\alpha$ there is an area size where the optimal pattern is switched.

In the example $\alpha=0.2$. The curves are drawn by using the fitted values of the measurements. As predicted, for small sizes interspersed patterns are favored because of having a lower coverage that overcomes the deficit caused by increased wiring cost. For larger sizes, this effect is reverted. With this value of $\alpha$ the transition between maps and interspersed patterns happens at approximately $10^{2} \mathrm{~d} m^{2}$. A different choice of parameter $\alpha$ shifts the transition region. This is shown in figure 5.3.1d). For every value of $\alpha$ there is a transition line marking the sizes where maps are optimal in green and where interspersed is optimal in red.

Taken together, the results show that the optimality theory proposed in this part of the thesis can produce a possible area size dependency on the type of the emerging layout in the cortex.

\subsection{Discussion}

The idea that the functional architecture in the visual cortex is shaped by the evolutionary drive to optimize an unspecified cost function was already proposed by Hubel and Wiesel [23]. The model 
proposed here is simple and thus easy to understand. The aim of the model is not to give a precise quantitative prediction for the exact critical size of V1, but to explicitly demonstrate the consistency of a plausible evolutionary mechanisms that could drive the evolution of the cortical architecture. The model shows a simple and plausible optimization principle that can explain the evolutionary divergence of V1 functional organization.

On the one hand the coverage of the system and its dependency on cortex size is a very robust feature that has only weak dependencies on the chosen parameters. This can already be inferred by the fact that optimization models for coverage lead to interspersed patterns [145].

On the other hand, the wiring cost is just one of different conceivable cost components that may favor a functional map architecture. Even if the variation between map realizations is removed, the difference between the wiring costs for maps and interspersed patterns depends strongly on the choice of parameters for the connection function. As was shown in the original paper [64], for maps with increasing number of Fourier modes to be optimal the orientation selectivity of connections between the neurons has to be very high. Intermediate values of $\kappa$ making the connection function cosine shaped lead to a stripe pattern as optimal solution. Although that kind of connectivity is more approximate to the one found experimentally in adult animals [115], using it in this approach leads to a decrease and then the inversion of the benefit in wiring costs for the different layouts, as neither maps not interspersed patterns are optimal in this case.

It is thus important to emphasize that wiring cost was used in this study just as an representative example that is conceptually simple. That this cost component leads to a change in optimal layout as the difference in coverage is reduced, is not a specific feature of wiring optimization. Any other optimization factor that favors maps over interspersed patterns and that is insensitive to area size will lead to a qualitatively similar theory and transition. The previous chapters of this thesis in fact suggest an alternative candidate. Also a selective value of the stability of the represented orientation of neurons would favor a map architecture over an interspersed design. The wiring cost was used in this study because it only relies on the structural properties of the layout and not its dynamics.

The view of area size as determinant for the functional layout has been questioned by the results from gray squirrel V1 [110]. They are highly visual rodents with a primary visual cortex of approximately the size of ferrets, but orientation maps are not developed. It is thus important to note that this result doesn't strongly refute the idea of area size as a critical factor influencing the functional organization. The above results show that the exact boundary marking the transition between the different layout types must be expected to be influenced by many factors. An example is the cellular composition of the visual cortex, which shows many differences between lineages. In rodents an increase in the number of neurons scales as a power law with area size, while in primates the scaling is linear [138]. This means that in an area with equal size the the total number of neurons is smaller in rodents that in primates. Another example is the difference in pyramidal cell layer thickness between the species [137]. The thickness is more than twice smaller in rodents compared to primates, suggesting a different proportion of cortical volume dedicated to intra-cortical connections. Therefore, to critically assess the area size hypothesis, it would be much more informative to examine species that are far from the possible transition region. These include on the one hand rodents with large brains, like the capybara with $300 \mathrm{~mm}^{2}$ V1 size [164], and on the other hand primates with small brains, like the mouse lemur with a V1 size of $10 \mathrm{~m} m^{2}$ [173]. 


\section{Chapter 6}

\section{Estimation of tuning curves in the rat visual cortex}

In chapter 4 interspersed patterns generated by simulations of the theory were analyzed. A robust prediction of the dynamical theory for the generation of interspersed layouts was that the preferred orientation of the neurons is very susceptible to noise. Even if the preferred orientation of some neurons is frozen in time, the remaining neurons continue fluctuating with almost the same diffusion coefficient as if every neuron behaved freely. The conclusion of the structural characterization was that the spatial arrangement of orientation preferences is not random, but has a negative correlation between nearest neighbors and an increase in the homogeneity of represented orientations (discrepancy) and stimulus responses (coverage). This chapter presents and applies methods to test those characteristic features in the visual cortex of rats.

How can these predictions be tested experimentally? The difference between the interspersed pattern and a random arrangement is very subtle, such that a very accurate estimation of the preferred orientation is needed. The estimation of preferred orientation, as any measurement, will inevitably involve a certain degree of variability. To characterize the lability of the tuning properties of neurons it is important to detect when changes actually occur and are not just a reflection of the uncertainty of the estimation. Therefore, to analyze the primary visual cortex of the rat the tuning curve of the neurons has to be estimated with a great precision and the confidence intervals of the fitted parameters have to be calculated to detect when changes are significant. Also, depending on the speed of change, the minimal number of observations needed to estimate significant changes of the tuning parameters in time needs to be determined.

In this chapter first the protocol of the 2-photon calcium imaging experiments in rat V1 is presented. The effect of two stimulation paradigms (episodic and continuous stimulation) and three fitting methods (vector average, Levenberg-Marquardt and Bayesian inference) on the estimation of the preferred orientation is then analyzed. The results show that, within the same stimulation paradigm, the orientation preference estimated with the different methods was very similar. The size of the confidence intervals however varied between the methods, with the smallest achieved by Bayesian inference. Therefore, from the estimation methods tested, Bayesian inference is the most statistically optimal. On the other hand, when the two stimulation paradigms were compared and the fitting methods maintained constant the preferred orientation and distribution of confidence interval sizes 
had a higher variation. This discrepancy indicates that the different stimulation methods have a strong impact on the response of the neurons.

Next the effect of the recording time on the accuracy of orientation preference estimation is addressed. The results show that a estimation of the tuning curve with great accuracy is possible with a relatively short presentation of the stimulus set. Interestingly, longer stimulation times do not lead to the statistically expected reduction of the confidence interval. To disentangle this effect, the data of a long recording from the rat V1 was split in equal parts and analyzed separately. Significant changes of the tuning between the parts were detected. In accordance with the theory, the tuning of some neurons was very variable, even if they were sharply tuned before and after the significant change in orientation.

This chapter of the thesis is a collaborative project with Jason Kerr from the Network Imaging group at the Max Planck Institute for Biological Cybernetics in Tübingen. The data acquisition and spike detection from the calcium signals was performed by Vishnudev Ramachandra.

\subsection{Experimental protocol and tuning curve estimation}

\section{Imaging protocol}

Lister Hooded male rats (>140g, P40-P49) were anesthetized by intraperitoneal injection of urethane (1.8g per $\mathrm{Kg}$ ). The binocular region of the visual cortex was identified by performing intrinsic optical imaging [40] on a $4 \times 4 \mathrm{~mm}$ area of thinned skull over the visual cortex [131]. A 2-3mm wide craniotomy was opened over the binocular visual cortex and sealed with Agarose $(1.2 \%$ in Ringer solution). Astrocytes were labeled with sulforhodamine 101 [174]. Neurons were bolus loaded by pressure injection of calcium indicator Oregon Green BAPTA-1 AM from pipette of tip size $1-2 \mu \mathrm{m}$. 2-photon imaging was carried out using mode-locked Ti:sapphire laser (MaiTai, Spectra Physics) at wavelength $920 \mathrm{~nm}$. Image acquisition was controlled using custom software. A CRT monitor (IIyama MT9021T, 1280X1024 resolution) placed 48cm in front of the rat was used for visual stimulation. Software for visual stimulation was written in Matlab with Psychophysics Toolbox extensions [175-177]. All procedures were performed according to the animal welfare guidelines of the MaxPlanck-Society.

\section{Stimulation paradigms}

Two stimulation paradigms are used:

- The episodic stimulation consisted of a moving square-wave grating of size 0.05 cycles/degree and drifting speed of 2 cycles/sec. Each grating was shown for 2 seconds with a inter-stimulus interval of 2-3 seconds. The luminance during the stimulus and inter-stimulus interval was $30 \mathrm{~cd} / \mathrm{m}^{2}$. A single stimulation trial consisted of the presentation of the moving grating drifting in 16 equally spaced directions in random order.

- In the continuous stimulation the square-wave grating of size 0.05 cycles/degree had two degrees of freedom: It drifted with speed 2 cycles/sec and rotated around the center of the screen 
with angular speed of $6 \mathrm{deg} / \mathrm{sec}$ [146]. A single stimulation trial consisted of one $360^{\circ}$ rotation of the grating.

\section{Data analysis}

Fast motion artifacts were corrected using the algorithm described in [178] and action potential evoked calcium transients were detected as in [179]. The binned temporal resolution of the spikecount was 0.1 seconds. The study focused on estimating orientation preference, such that the direction of the stimulus was divided modulo $180^{\circ}$. For episodic stimulation the spikes recorded during each 5 bins were added, representing one complete movement of the dark-light or light-dark edge of the grating. For each neuron a stimulus-response vector was generated and was used to fit a tuning curve with the three different methods discussed below. To obtain the confidence intervals of the fitted parameters the bootstrap method was used [180]. In this approach the parameters are fitted multiple times with stimulus-response pairs re-sampled with replacement from the measured data. The 2.5 and 97.5 percentiles calculated from the samples are the bootstrap confidence intervals. For the estimation of the tuning parameters for each sample three different methods were used:

- Vector averaging: The magnitude of each response is multiplied by an unit vector of angle twice the orientation of the corresponding presented stimulation and the obtained value is then averaged over all stimulus-response pairs. This procedure is most transparent for a cosine tuning curve

$$
T C(\phi)=A_{0}+A \cos (2(\phi-\theta))
$$

Then the amplitude $A$ corresponds to the absolute value of the vector average and the preferred orientation $\theta$ to half the argument of the vector average. The preferred orientation is independent of the mean response $A_{0}$. In the text this method will be abbreviated as VAV.

- Levenberg-Marquardt fitting [181]: This iterative method starts from an initial guess of the tuning curve parameters and modifies them in order to minimize the sum of square errors between the data and the predictions of the model. When the sum of squares of errors is large the method acts similar to gradient-descent methods, where the parameters are updated in the direction of the greatest reduction of the least squares objective. As the error is reduced the method acts similar to Gauss-Newton method, where it is assumed that the least squares function is locally quadratic and the parameters are updated to find the minimum. The tuning curve to fit was a Von Mises function

$$
T C(\phi)=A e^{\sigma \cos (2(\phi-\theta))}
$$

where $A$ is the amplitude of the tuning curve and $\theta$ the preferred orientation. The parameter $\sigma$ regulates the width of the tuning curve; for constant $A$ and $\theta$, the larger $\sigma$ the sharper and higher the peak of the tuning curve will be. The initial guess for the parameters $\sigma$ and $\theta$ were respectively the magnitude and orientation of the vector average, and for the parameter $A$ the maximum average response for all stimulus orientations was taken. The fit was labeled as successful when either the error, the gradient or the change in the parameters reached a value smaller than $10^{-7}$ in an iteration. Unsuccessful estimates happened when 100 iterations were completed without reaching a solution satisfying the requirements above or when the Jacobian 
matrix was numerically not invertible. Unsuccessful bootstrap samples where not considered for the confidence interval. In the text this method will be abbreviated as LM.

- Bayesian inference [147]: This method evaluates for a set of $\mathrm{k}$ parameters the posterior probability of the tuning curve being consistent with the measured data given a prior assumption about how the neuron response relates to the tuning curve (e.g. Poisson or Gaussian) and the relation between the tuning parameters. This generates a k-dimensional probability field of tuning curves. A Markov Chain Monte Carlo method [157] is used to make a random walk in this parameter space to approximate the distribution of tuning curves in it and to find the maximum posterior probability. The tuning curve to be fitted with this method was a circular Gaussian function

$$
T C(\phi)=B+A \sum_{n=-\infty}^{\infty} e^{-\frac{(\phi+180 n-\theta)^{2}}{2 \sigma^{2}}}
$$

where $A$ is the amplitude, $\theta$ the preferred orientation and $\sigma$ the width of the tuning curve. The parameter $B$ determines the magnitude of the minimum response of the tuning curve. The relation of spikes to the tuning curve was chosen to be Poisson distributed, where the mean of the distribution for a given orientation $\phi$ is the corresponding value of the tuning curve. The posterior probability of the obtained tuning curve is divided by the posterior probability of a constant tuning curve $T C(\phi)=c$, a measure called Bayes factor. It measures how much better the data is explained by the circular Gaussian than by a constant "tuning curve". The software for this method was first provided in [147]. Corrections to the circular statistics in the calculation of the bootstrap confidence intervals and the possibility to modify the seed of the random numbers in the program were added in this work and shared with the authors. In the text this method will be abbreviated as BAY.

It is important to note that although the parameters $A$ and $\sigma$ of the different models represent the same concept, they are not mathematically equivalent and can't be directly compared. However, direct comparison is possible for the preferred orientation of the tuning curve $\theta$, which is equivalent in all models and is our wanted read-out. Since the preferred orientation $\theta$ is estimated from different tuning curve functions, the comparison on the size of the confidence interval has to be done with care.

\subsection{Comparison of the stimulation and estimation methods}

The activity of 86 neurons corresponding to four fields of view at different depths was recorded while presenting 7 repetitions of one episodic stimulation trial followed by one continuous stimulation trial. The results of estimating the orientation preference with the different methods is shown in figure 6.2.1. Each point in the plots corresponds to the orientation preference as estimated by the methods labeled in the $\mathrm{x}$ and $\mathrm{y}$ axes. The color of the axis represents the stimulation paradigm used. Episodic stimulation is labeled with blue and continuous stimulation with orange. Even if the orientation of the neurons are not on the diagonal for the pair of fitting methods, the difference between the estimates can be unsignificant. In the plots, non-significant differences are labeled in green and significant differences in red. The average absolute orientation difference in the estimated orientation preferences is marked in the corner of each plot. 
a
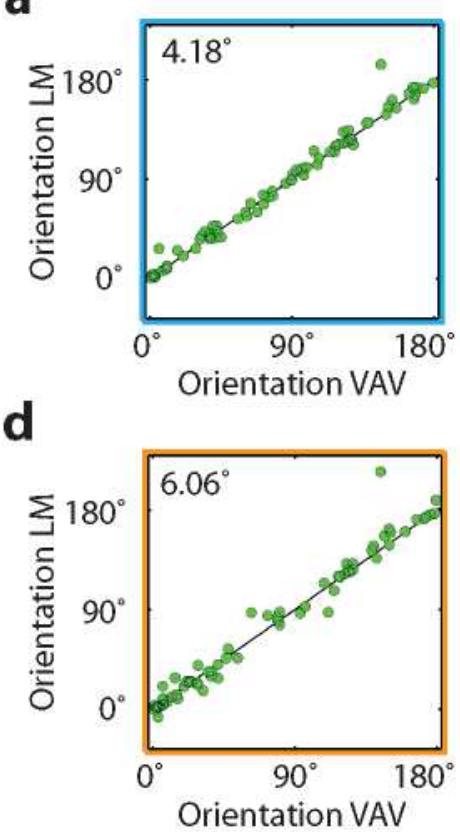

g

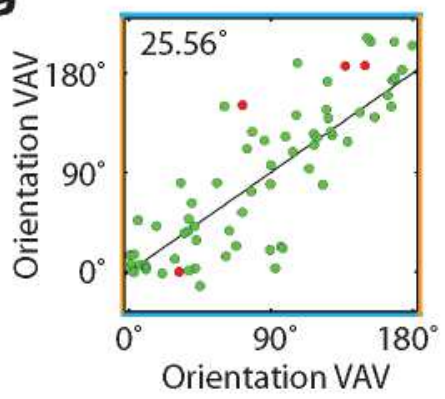

b

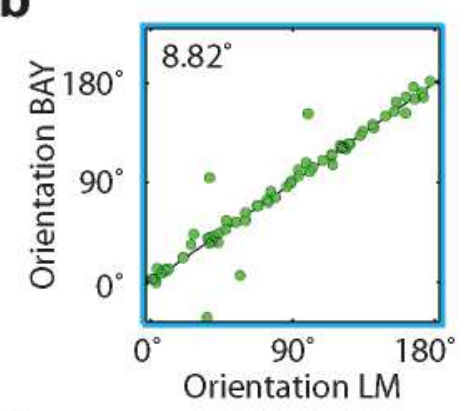

e

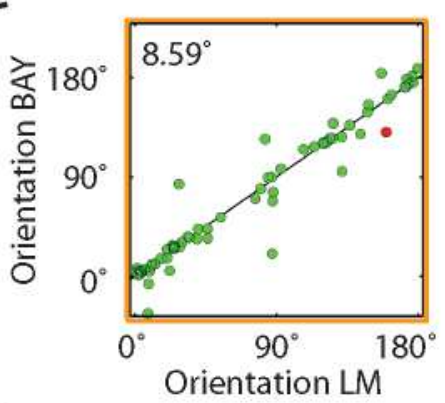

h

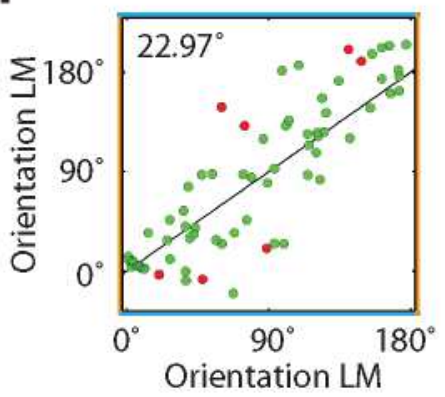

C

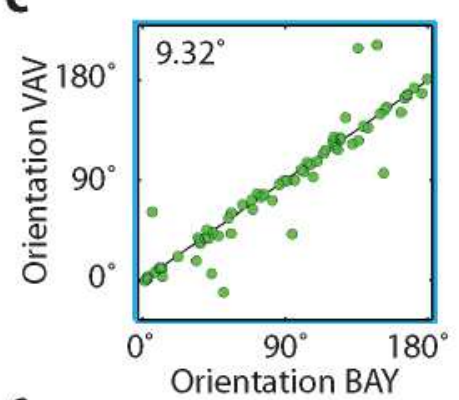

f

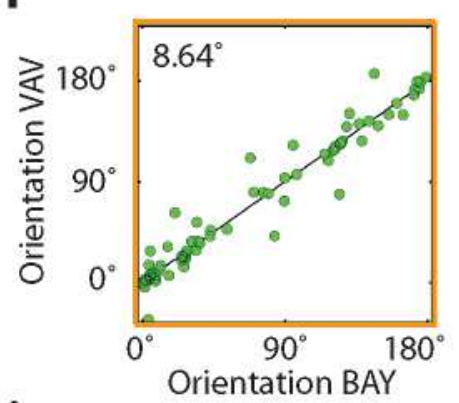

i

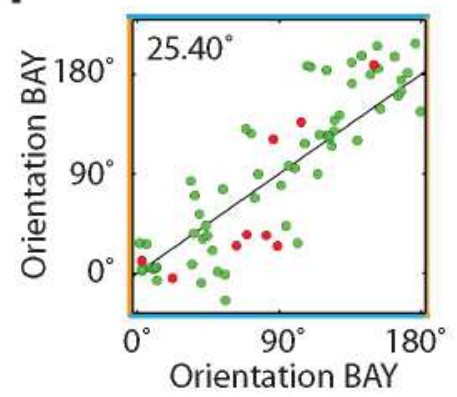

Episodic stimulation $\square$ Continuous stimulation Difference not significant Difference significant

Figure 6.2.1: Comparison of the estimated preferred orientation from episodic and continuous stimulation (blue and orange borders respectively) using different estimation methods: vector averaging (VAV), LevenbergMarquardt fitting (LM) and Bayesian inference (BAY). The mean absolute orientation difference is displayed for each comparison. Green markers represent that the difference in the estimated orientation is not significant and red markers represent significant differences. Comparisons within one stimulation paradigm show that the estimated orientation does not significantly differ with the different fitting methods. Cross-stimulation comparisons are more variable, indicating that the response of the neurons is different depending on the method used. 
a

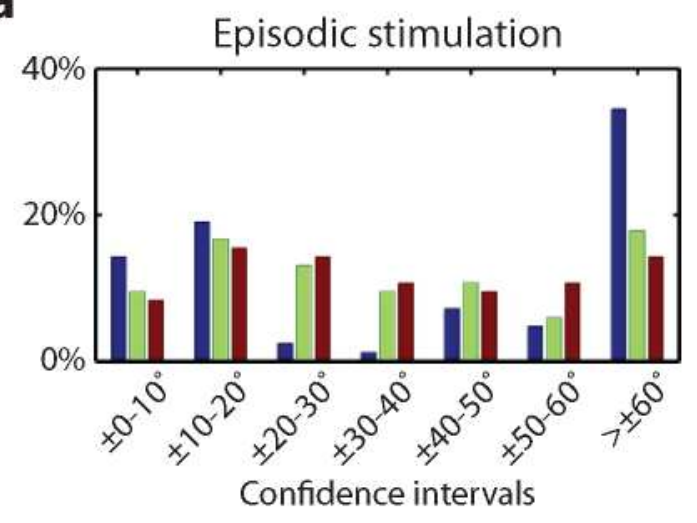

b

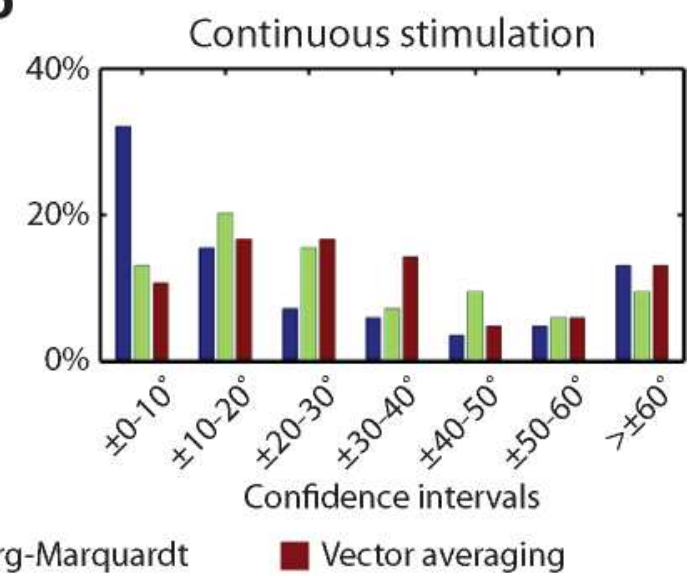

Figure 6.2.2: Histogram of orientation confidence interval sizes for the different stimulation and estimation methods. Blue bars correspond to Bayesian inference, green bars to Levenberg-Marquardt and red bars to vector averaging. In a) episodic stimulation is used and in b) continuous stimulation. The highest number of small confidence intervals is obtained when using Bayesian inference.

In figures a) to c) episodic stimulation is used. The estimated orientation is consistent across fitting methods, specially between VAV and LM. This is to be expected because the LM method uses the results of the VAV as initial guess for the parameters. In figure d) to f) continuous stimulation is used. The estimated orientation preference is also consistent across methods. The distribution of preferred orientations is different as for the episodic stimulation, having a larger clustering around $0^{\circ}$. This will be analyzed further below. That the estimated orientations are different can be seen in figures g) to i), where the orientations for the different stimulation paradigms using the same methods are compared. The difference between the orientations is much larger, with many neurons having a significant difference. Since the stimulation paradigms are different, not necessarily all the neurons will have the same response for both of them. One neuron might be highly selective for a specific orientation that is not presented in the episodic stimulation, resulting in a lower selectivity in the later. Similarly, some cells might respond only to strong changes in the receptive field, and therefore since in the continuous stimulation the presentation of the grating is not interrupted during the whole trial those neurons will have a lower response than with episodic stimulation. With episodic stimulation the neurons are strongly driven by the sub-cortical afferents, such that most fire at the onset of the stimulus. In comparison, with continuous stimulation the initial high activity reaches equilibrium, such that the activity elicited by recurrent networks is accentuated.

Independent of the cross-stimulus comparison, the results show that estimating the tuning curve with methods of increasing complexity doesn't lead to a significant difference in the orientation preference. How do the confidence intervals depend on the used method?

Figure 6.2.2 shows the histogram of confidence intervals for the different fitting methods; BAY in blue, LM in green and VAV in red. Figure a) shows the results of episodic stimulation and figure b) for continuous stimulation. In figure a) it is shown that to estimate confidence intervals below $20^{\circ}$ the fitting method plays a role, where a higher number of small intervals is obtained with decreasing order by BAY, LM and VAV. This difference might depend both on the complexity of the fitting method or the number of parameters used to model the tuning curve. Preliminary analysis using a circular 
a

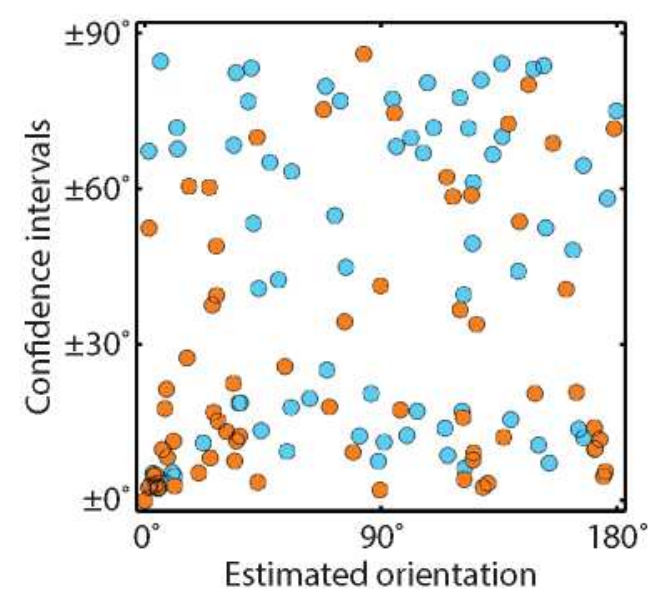

b

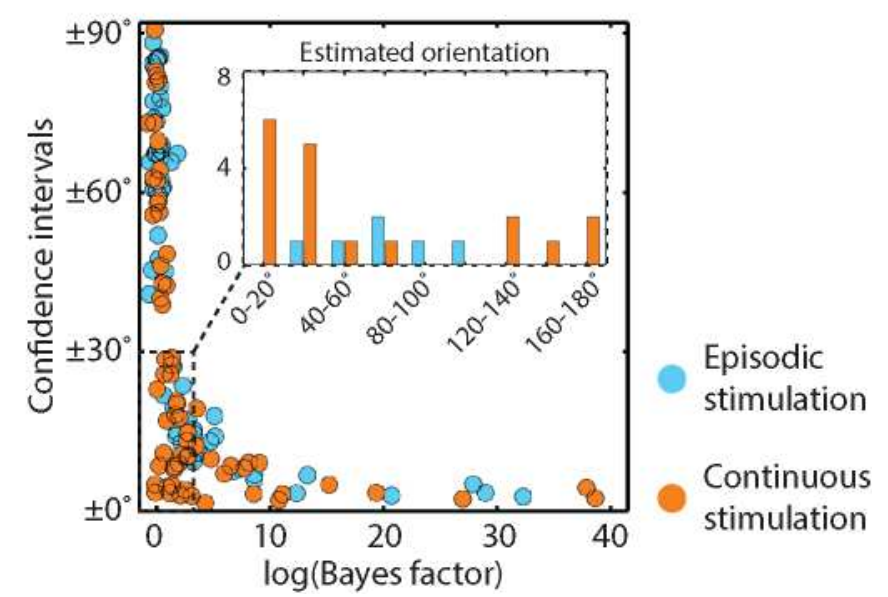

Figure 6.2.3: With continuous stimulation orientation preferences close to $0^{\circ}$ are more frequent compared to episodic and have a smaller confidence interval. a) Size of the confidence intervals as a function of estimated preferred orientation. b) Size of the confidence intervals as a function of the Bayes factor. Inset: Histogram of estimated orientation preferences with confidence intervals below $30^{\circ}$ and Bayes factor below 100. In both plots episodic stimulation is marked with blue and continuous stimulation with orange.

Gaussian with LM to have a direct comparison with BAY show that the number of parameters is not the main cause of the reduction of the size of the confidence interval. This preliminary result depends on the initial conditions of the parameters in LM, so it is possible that a better initial guess leads to a higher match between the methods. In the BAY algorithm the random walk in parameter space removes the impact of the initial guess.

The distribution of confidence interval sizes for LM and VAV behaves similar, with all sizes more or less equally represented. On the other hand, in BAY there is a tendency to either estimate small or large confidence intervals, with intermediate intervals under-represented. This shows that BAY is more susceptible to bootstrap samples for neuron that are less orientation selective, doing a better job in detecting them. Therefore BAY is not only theoretically optimal but also conservative in assigning large confidence intervals to weakly tuned neurons.

For the continuous stimulation in figure b) the same conclusion about the efficiency of the methods in estimating small confidence intervals apply. Interestingly, for BAY there is a large fraction of neurons with a very narrow confidence interval. As mentioned before this could be caused in part by the difference in response properties of the neurons to the different stimulation paradigms. On the other hand, this effect might be caused in part by details of the stimulation paradigm that can be corrected. A hint of the second is the over-representation of estimated orientations close to $0^{\circ}$. This is analyzed for the BAY approach in more detail in figure 6.2.3. In figure a) the size of the confidence intervals as a function of the estimated orientation is plotted, where blue marks episodic and orange continuous stimulation. As found before, for continuous stimulation there is a clustering of orientations close to $0^{\circ}$, where many of them have a very small confidence interval. This is not the case of episodic stimulation, with a more homogeneous distribution of orientations and confidence intervals. In figure b) the confidence intervals as a function of the Bayes factor of the estimation is plotted. The Bayes factor gives the ratio of the posterior probability of the data being described by a circular Gaussian versus 
being described by a constant function. For episodic stimulation in blue the expected behavior is obtained; with higher Bayes factor the neurons are more likely orientation selective and have therefore a decreasing size of the confidence interval. For continuous stimulation in orange the overall behavior is similar, but there is a larger amount of neurons having both a low Bayes factor and a low confidence interval. The inset in the figure shows the distribution of estimated orientations for the neurons having a Bayes factor lower than 100 and confidence intervals smaller than $30^{\circ}$. The inset shows that the majority of estimates having those conditions come from continuous stimulation and that most of them have an orientation preference close to $0^{\circ}$. What makes the horizontal orientation special?

The over-representation of this orientation does not depend on the fitting method, as it is present with the three algorithms used. One possible reason for this effect is that the rotating grating is drifting at this orientation at the beginning of the stimulus, causing an increase in the response due to the onset of the stimulus. A solution for this is to start the drifting of the grating at a random orientation in each trial or to complete a $366^{\circ}$ turn and neglect the first recorded bins. A second possible cause is that the calcium indicator used to detect activity in the neurons might have a weaker response in time due to the continuous stimulation, making spike detection less accurate. This possibility has to be tested by comparing electrophysiological recordings with the spikes obtained from the calcium imaging. If this is found to be a problem, a solution is to make breaks during the continuous stimulation for the signal to recover. Since those experiments and tests are yet not completed by the experimental collaborators, the rest of this chapter uses data from episodic stimulation only.

Taken together the results show that small confidence intervals on the order of a few degrees can be obtained with all estimation methods. In the next section the length of the recording necessary to obtain small confidence intervals is analyzed.

\subsection{Optimal stimulation time}

The experiments in rats require the injection of a calcium indicator to the cortex. With time the signal obtained by the indicator decays, defining a temporal window where the response of the neurons can be recorded. Therefore, if a large number of neurons has to be characterized with high accuracy, the time spent in recording every field of view has to be optimized. An appropriate way of characterizing the effectivity of the recording is the size of the confidence intervals for the preferred orientation. Theoretically, the longer the sampling, the smaller the confidence intervals will be with an expected decrease proportional to the inverse square root of the sample size. In reality, correlated noise and fluctuations in the response of the neurons will influence how accurately the preferred orientation can be estimated. If orientation preference is not really stationary, the confidence interval size may saturate or even increase for longer recording times.

In this section 78 neurons corresponding to 3 fields of view at different depths were recorded during the presentation of 9 pairs of episodic and continuous stimulation trials. Progressively increasing lengths of the response were used to fit a tuning curve as described above. Figure 6.3.1 shows the result of this approach. Only the results for the episodic stimulation are shown. In each plot the colors correspond to different lengths of the recording as labeled at the bottom of the figure. Each plot shows the cumulative probability of finding neurons with a confidence interval size below a certain value. For example, if the curve passes at $[ \pm 30,0.4]$ means that $40 \%$ of the neurons have a 

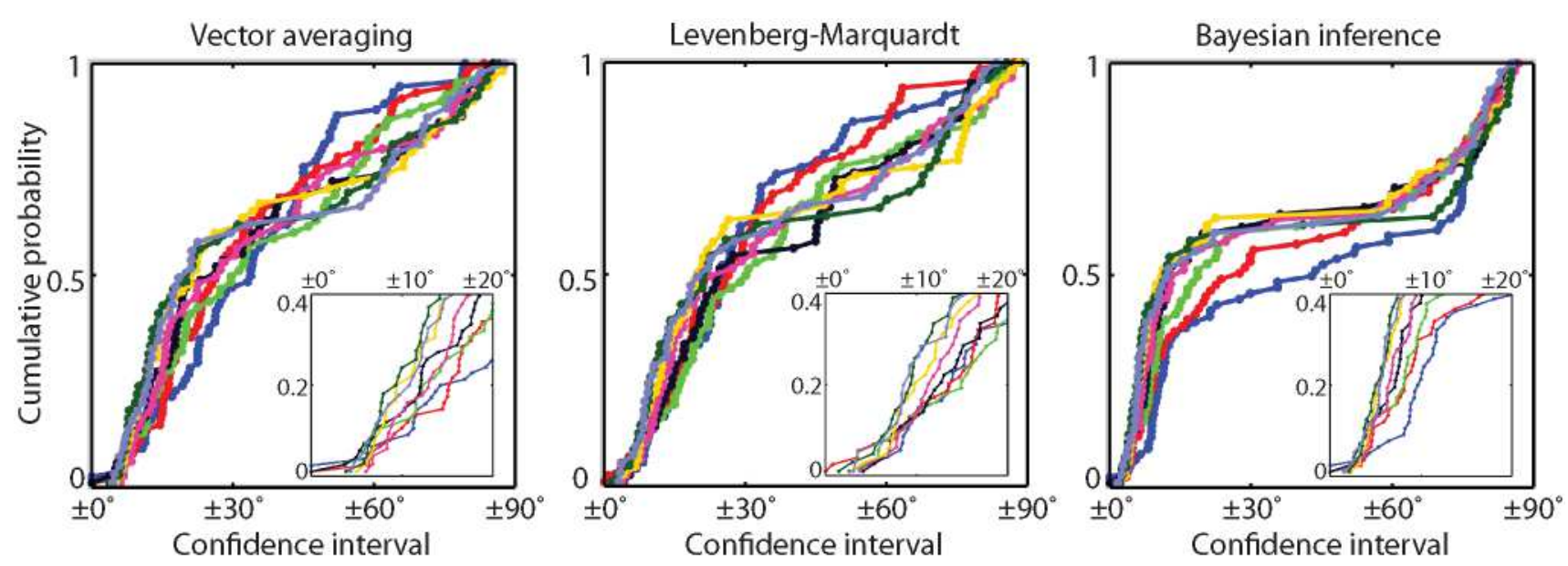

Stimulus trials presented: $\because 1=2=3 \quad 4=5=6=7=8$

Figure 6.3.1: A small number of stimulus trials are necessary to have a good estimation of the preferred orientation. Each plot shows the probability of finding neurons with a confidence interval below or equal a certain size. The colors correspond to increasing numbers of stimulus trials presented. The estimation method used from left to right was vector averaging, Levenberg-Marquardt and Bayesian inference. The insets correspond to a magnification of the curves for small confidence intervals.

confidence interval size below or equal to $\pm 30^{\circ}$. The inset in the figures is a magnification of the curves for small confidence intervals.

The results show that as the number of stimulation trials increases the orientation of the neurons is estimated with smaller confidence intervals. After a few trials this improvement represents only a few degrees. In VAV confidence intervals are quickly improved with more presented trials, while for LM this only happens for longer stimulation time. The steepness of the curve is highest with BAY, such that after 3 trials the number of neurons with a small confidence interval is larger than for LM and VAV after 8 trials. Interestingly, with only 2 presentations with LM and VAV small confidence intervals are not very common, and longer stimulation time is needed to increase their number. This is not observed in BAY. There, the bi-modality described before is achieved with 4 trials.

Taken together, these results show that a small amount of presentations are sufficient to have a good estimation of the preferred orientation of the neurons. Since the most precise estimation method is BAY, it will be used in the rest of this chapter.

\subsection{Non-stationarity of the preferred orientation}

According to the simulations in chapter 4, interspersed patterns are predicted to be very susceptible to noise-induced random drifts of preferred orientation. In this section the stability of the estimated preferred orientation in the rat visual cortex is addressed. In the previous section it was shown that long stimulation times are not necessary to have a good estimation of the orientation preference. Therefore, the recoding of an experiment is split in parts and the tuning curve is estimated using Bayesian inference independently for each. The question to answer is if the estimated orientation changes significantly during the course of a recordings, which takes less than 30 minutes. 
a Data split in 3 parts with equal amount of time
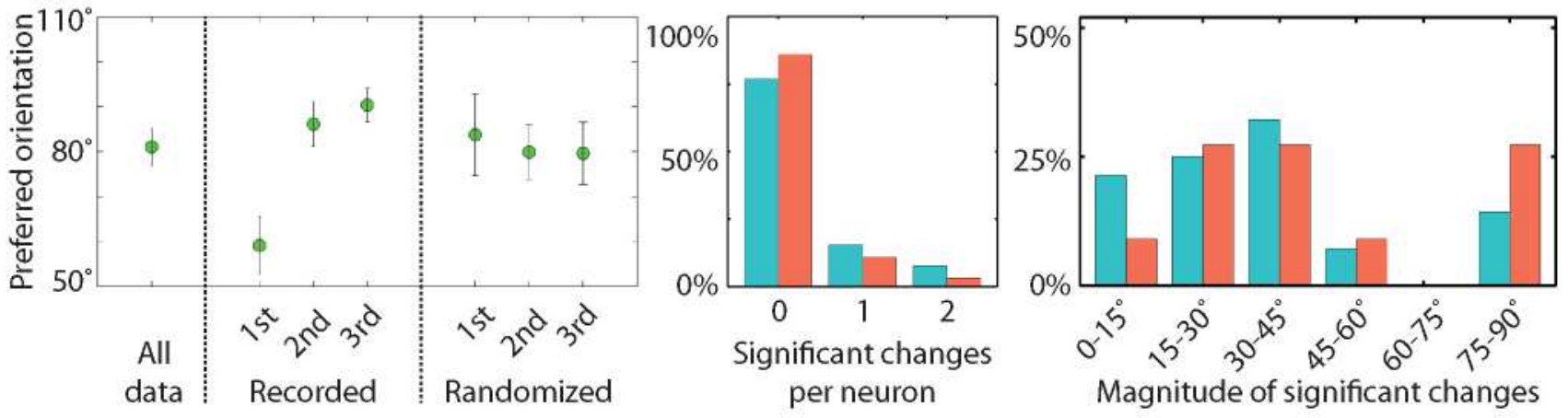

b Data split in 3 parts with equal amount of spikes
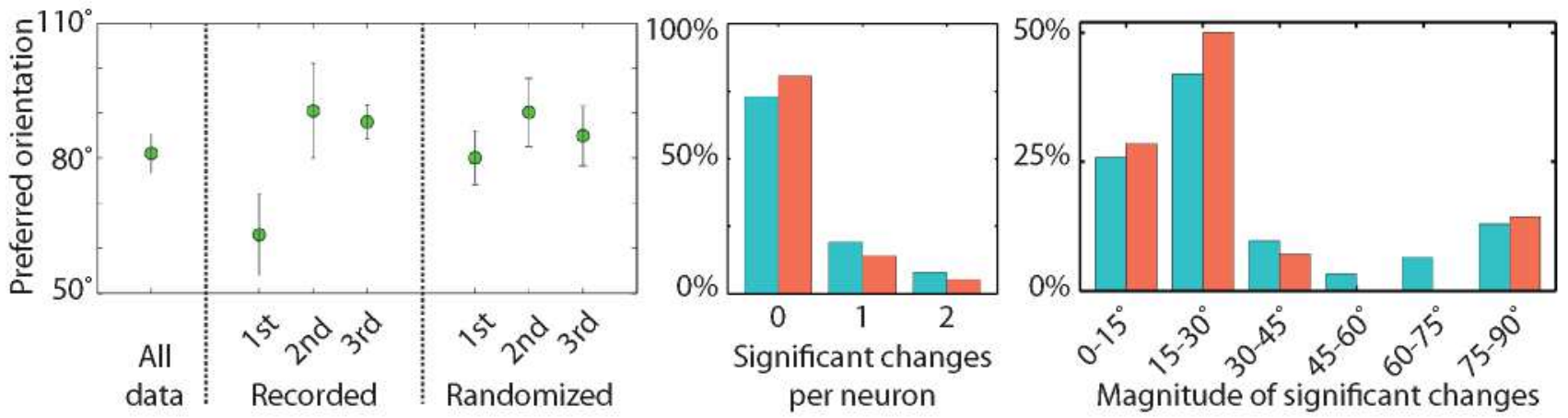

OSI $>0.5$

OSI $>0.5+$ no change in randomized comparisons

Figure 6.4.1: The preferred orientation in some neurons is not stationary. The figures show the results of dividing the episodic recording of one experiment in 3 parts with equal amount of time in a) and spikes in b) and comparing the preferred orientations estimated with Bayesian inference. In the plot on the left the estimated orientation in the different parts for one example neuron are shown. During the course of the experiment, the neuron significantly changed its orientation. In the division in parts of the randomized data this change was not present. Next to the example neuron are the fraction of significant orientation changes in the population and an histogram of the size of the changes. Only comparisons where the estimated tuning had an OSI $>0.5$ before and after the change were considered (blue columns). Red columns represent the distribution when the extra condition is imposed that the orientation of the neuron doesn't change in any of the randomized comparisons. 
For this analysis the recording of 102 neurons in 4 fields of view during the presentation of 8 sets of episodic and continuous stimulation trials was used. This experiment was chosen because the number of selective neurons was high. The recording of the episodic stimulation was split in 3 parts in two different ways: i) with equal amount of recorded time and ii) with equal amount of spikes. The first is used to analyze if a neuron changes its orientation in time and the second to control if the change is caused by a decrease in firing rate during one of the parts leading to a different estimation. It is important to keep in mind that this recorded time and spikes is the combination of the 8 sets of episodic trials and explicitly neither includes the inter-stimulus-interval of the episodic stimulation nor the data from the interleaved continuous stimulation. As a control the order of the recording was randomized 10 times and the results were also divided in 3 parts as described above. The goal of this is to remove the temporal structure of the recording and detect if the significant change could have happened by chance because of fluctuations of the signal. The estimated preferred orientations in each part were compared and only neurons where the selectivity of the tuning was high in both compared parts were taken into account. The selectivity of the tuning was quantified with the orientation selectivity index (OSI), that using the tuning curve estimated with Bayesian inference in equation 6.1.1 can be defined as

$$
\text { OSI }=\frac{T C(\theta)-T C\left(\theta+90^{\circ}\right)}{T C(\theta)+T C\left(\theta+90^{\circ}\right)}=\frac{A}{A+B}
$$

Only neurons with OSI>0.5 in both compared parts were used.

Figure 6.4.1 shows the result of this procedure for the data split in parts with equal amount of time in a) and spikes in b). Left is the results of the same example neuron with both procedures, where the $x-$ axis marks the part the data is divided into and the y-axis is the corresponding estimated orientation preference. The confidence interval of the estimated orientation are shown as error bars.

The plot in a) shows how the orientation is significantly different in the first part compared to the second and the third, while the second and third are the same within the confidence intervals. From the division in spikes in $b$ ) it can be seen that this change in orientation is not caused by a decrease in spiking during one of the parts. In all the randomized samples (only one shown) this significant change doesn't occur, showing that the precise order of the spikes in the recording is necessary.

The results in the population of neurons are as follows: For the data divided in time, 91 neurons were selective in at least one pair of compared parts and 21 neurons showed at least one significant change of orientation in those comparisons. If the condition of stationarity in all randomized parts is included, the number of neurons satisfying both conditions reduces to 64, out of which 9 neurons undergo at least one significant change. For the data divided in spikes, 89 neurons were selective in at least one pair of compared parts and 24 neurons showed at least one significant change. Including the second condition, the number of neurons satisfying them reduces to 57, out of which 11 neurons undergo at least one significant change. The plot in the middle in figure a) and b) summarizes this results, showing the percentage of neurons that undergo zero, one or two significant changes during the comparisons. The bar in blue only uses as requirement that the neuron has to be selective in both compared parts and the red bar also requires the orientation to be stationary in the comparison of all randomized parts. About $20 \%$ of all the neurons that satisfy this strong requirements changes their preferred orientation at least once, either if the data is split in time or in spikes.

The plots in the right show the histogram of the distribution of sizes of the significant changes of orientation in the compared parts. Although most changes are below $30^{\circ}$, it is surprising that in 
a
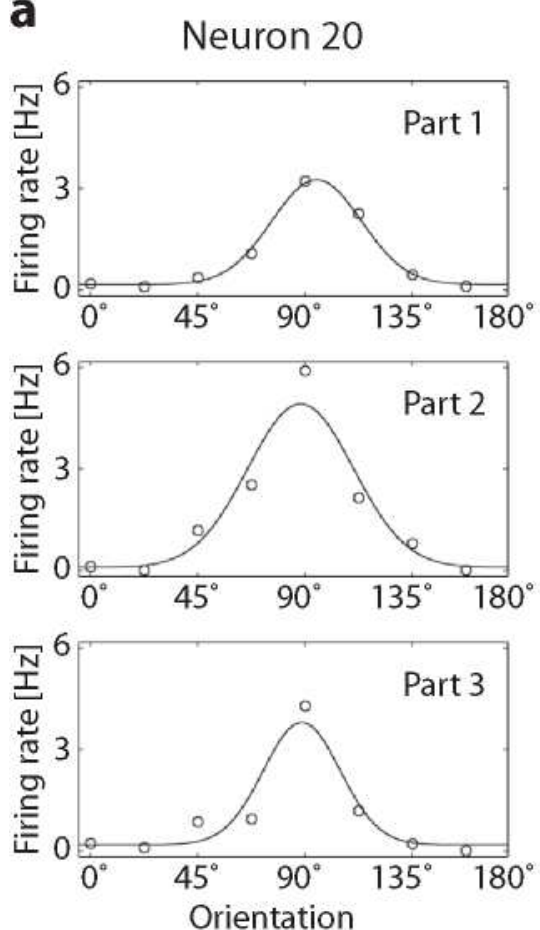

b
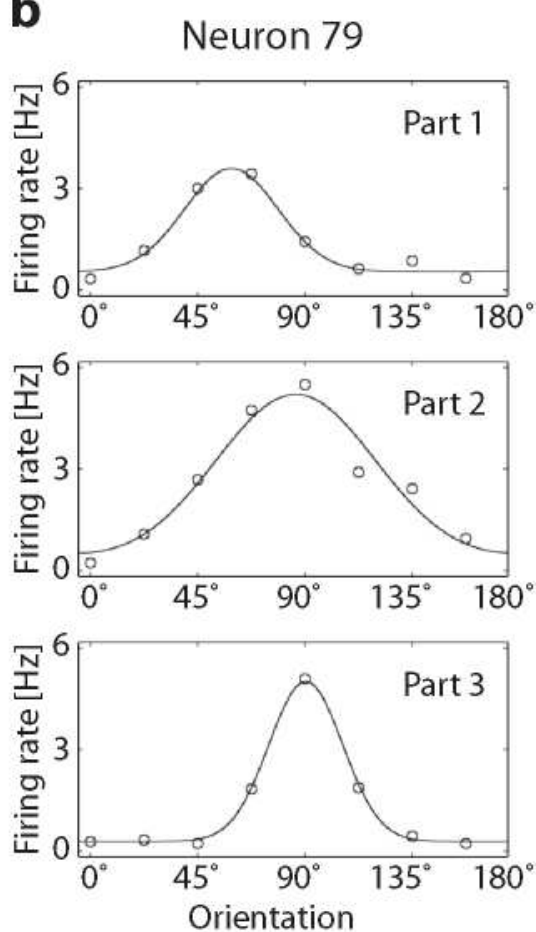
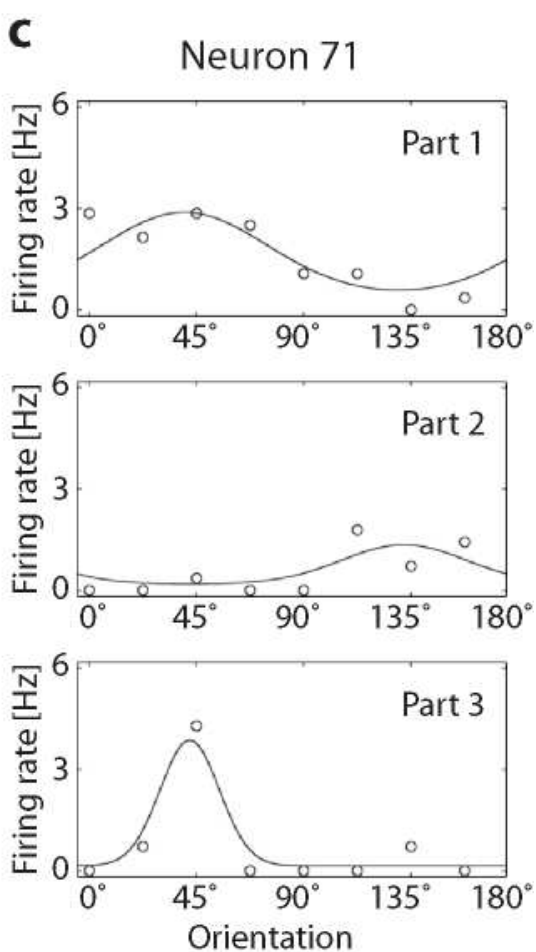

Figure 6.4.2: Stability and lability of the preferred orientation in a fraction of neurons. The data is split in three parts with equal amount of recorded bins, presented in the top, middle and bottom rows. In all plots the circles correspond to the mean firing rate from the recordings and the lines to the estimated circular Gaussian using Bayesian inference. a) Example of a neuron with consistent orientation preference during all subdivisions. b) Example of a neuron that exhibits significant changes between the first part and the rest of the subdivisions (same neuron as in figure 6.4.1a)). c) Example of a neuron that has a drastic change in orientation preference in the second part of the recording.

the course of the experiment some neurons can change their orientation almost by $90^{\circ}$. Figure 6.4 .2 shows the estimated tuning curves in each subdivision for three example neurons. The neuron in 6.4.2a) had a stable orientation preference, where only the orientation selectivity fluctuated in time. This neuron is a representative of the majority of neurons recorded during the experiment.

Figure 6.4.2b) shows the same neuron used for the example in figure 6.4.1. The preferred orientation in the first part of the recording was significantly different from the rest. The neuron developed a high selectivity to an orientation for which it was initially not very responsive. With a mean orientation shift of $28^{\circ}$, this case represents the majority of neurons where a significant change in the orientation preference can be detected. In figure 6.4.2c) a neuron is plotted that had a very high change in orientation preference. In the first two parts of the experiment this neuron is not as selective as the other examples. Even then, it can be appreciated that the response to the preferred orientation is silenced during the second part of the experiment, leading to a drastic change in orientation preference. This change is reversed in part 3 of the experiment.

The results show that the orientation preference of at least a fraction of neurons in the rat visual cortex are not stationary, but that it can fluctuate during the course of an acute experiment. The combined requirement of high selectivity and stationarity of the randomized recording is very strong test on 
the lability of the neurons. The idea here is to show that even with these conditions some neurons are significantly changing their orientation.

\subsection{Discussion}

In this chapter it was shown that the preferred orientation including confidence intervals can be estimated quite precisely in 2-photon calcium imaging experiments. The confidence intervals can be very small, and a very long recording is not required to have a good estimation of the tuning parameters. A combination of episodic stimulation and Bayesian inference of the tuning curve gave the best results both in the number of neurons with small confidence intervals and the number of stimulus presentations required to measure them. Continuous stimulation was found to induce a bias in the estimated orientation of the neurons. Once this stimulation method is optimized, the recording time might be further reduced and the confidence intervals made smaller. It was shown that the orientation of some neurons was not static, but had significant changes during the course of the experiment. These changes happened sparsely and at different moments of the recording, making it unlikely that they are caused by eye movements or eye rotations in the anesthetized animal. These preliminary results supports the predictions of the theory about the dynamics of preferred orientation in an interspersed organization, where the orientation preference of the neurons is predicted to be sensible to noise. Once the recording protocol is completely optimized to reduce recording time without impairing the accuracy of the estimation, a large number of neurons can be recorded and the spatial structure of the disordered pattern can be addressed. The use of 2-photon calcium imaging is encouraged since in this method the individual neurons can be fully distinguished the eliminating error sources coming from recording from two distinct neurons.

The significant changes of orientation preference in the relatively short time-frame poses many interesting questions. What is the role of the continuous stimulation presented in-between the recordings of the episodic stimulation? Are the orientations of the neurons also changing when no continuous stimulation is shown? What would be the results of presenting naturalistic movies to the rat in-between the episodic stimulation trials? It is expected that the changes in orientation preference are caused by the activity of the surrounding neurons. It is therefore interesting to quantify how the presented results vary with different stimulation types in-between the recordings.

Other question that arises is if those fluctuations in orientation are also found in neurons inside an orientation map. Although it is possible that individual neurons change their orientation over time without external manipulation, the overall order of the maps is known to be quite robust [182]. Fluctuations would have to keep in the vicinity of the population surrounding the neurons such that the pattern is not lost. The orientation preference of individual neurons in cats can be modified by pairing the desired orientation with external manipulations of the firing of the neurons [98, 183]. It can be addressed how long this modifications last under normal cortical activation (e.g. naturalistic movies) and if the preferred orientation of the neurons returns to its initial value corresponding to the column they belong to. Changing receptive field properties by imposing activity is also possible in rats [184, 185], although a modification of the preferred orientation as in the previous experiments hasn't been performed. According to the theory and the results presented here, changing the preferred orientation of the neurons in rodents must be far easier than in animals with maps, with a smaller strength and number of stimuli needed. 
The results show that the the majority of neurons have a robust orientation preference, at least with the conditions used to distinguish when significant changes occurred. Is there any difference between the neurons that are labile and the ones that are not? As described in [124], neurons responding to similar visual features become preferentially connected as the cortex develops. This stronger connectivity between like-to-like might reduce the variability of the orientation preference. The simulations show that the fluctuations of the rest of the cortex is not impaired by having the orientation of some neurons fixed in time.

The preliminary results described above are obtained from an experiment that was designed to assess and optimize the accuracy of preferred orientation measure. Based on our preliminary findings experiments are being planned to directly test the predictions of the model about the lability of neurons. In those experiments only one stimulation method is used and the neurons are recorded for a longer time. Also the possibility of eye movements is removed by fixing the eye of the anesthetized rat during the experiment. To have longer recordings, the use of transgenic mice can be considered, where the calcium indicator is genetically encoded and the time window for the recording is vastly expanded. In this case the stability and changes of the tuning curves under normal visual experience in-between the recordings can be addressed. 


\section{Chapter 7}

\section{Summary and discussion}

In this thesis the class of dynamical models introduced by Wolf [20-22, 186], that is currently the only modeling approach capable of generating orientation maps in quantitative agreement with the geometry of maps found in primates and carnivores, was generalized to investigate conditions for the emergence of disordered orientation preference layouts, as found in rodents and lagomorphs. The predicted pattern is determined by the shape of the intra-cortical interactions. As in the previous model, ordered pinwheel-rich maps emerge as solution when the short-range interactions are Mexican-hat-shaped and long-range orientation-selective interactions are present. In contrast, with strong, local and unselective inhibition disordered arrangements are obtained. It is important to emphasize that the dynamical model by itself has no intrinsic disorder, since neurons are densely interacting with their neighbors and the tuning dynamics are exactly equivalent for all neurons. The disorder in the system is obtained dynamically. As an emergent collective phenomenon even ordered initial conditions become disordered in time. The role of strong inhibition in generating disordered arrangements was confirmed by implementing it in a correlation model based on Hebbian competition in the LGN-V1 synapses [53]. With strong inhibition oriented receptive fields form in this model, but their orientation is disordered in space.

The order-parameter model was further expanded to have two orientation preferences representing the inputs from the two eyes. A complete parametrization of such models up to 4 th order energy terms is derived. The late binocular orientation matching after the emergence of orientation selectivity as found by Wang et al. [127] is only obtained in the model when specific intra-neuron nonlinear coupling mechanisms are included. Interestingly, this mechanism that increases the order among the neuronal inputs can coexist with the dynamical disorganization of the layout for different obtained by the strong inhibitory inter-neuron interactions. This means that even with a high degree of orientation change to match the orientation preference of both eyes the interspersed pattern is still generated. By implementing this binocular model in simulations representing the development of a PSD-95 knock-out it was shown that the abstract parameter model can be tuned to represent experimental time and changes in tuning dynamics during the critical period. The results of this implementation predict that the binocular matching in PSD-95 knock-out mice is impaired by the decrease in selectivity of the neurons [153], even if the critical period is prolonged [154].

The interspersed layout that develops in the simulations, however, is not random. A negative correlation between the nearest neighbors can be measured. This signature of dynamical generated 
disorder scales inversely with the number of neurons interacting with each other and is therefore most likely small. It was found that random patterns with the same correlation function obtained by the dynamics are themselves not solutions. The interspersed arrangement is further characterized by higher order correlations. First, based only on the spatial arrangement of preferred orientations, the obtained layout is shown to have reduced values of discrepancy, which measures deviations in the homogeneity of the represented orientations for any subregion of the cortex. Secondly, based on the functional properties of the neurons in such an organization, the interspersed pattern is shown to have an improved stimulus coverage, which measures the homogeneity of the evoked cortical response by the presentation of any possible stimulus. Both characteristics are improved in the obtained patterns compared to ordered maps, random orientations and negatively correlated random layouts. In agreement with the coverage result, in a stimulus driven optimization model based on Hebbian learning of input representations it is shown that the layout that maximizes this quantity is disordered.

The dynamics of a neuron in an ordered and disordered pattern is also different in the simulations. In interspersed patterns the tuning of the neurons is more labile, such that small amounts of noise can induce high variations in their orientation preference. In ordered maps this is not the case, since the impact of the noise is balanced by the dynamical drive of the neurons to match the orientation of their neighbors. This high lability of tuning parameters in neurons in interspersed patterns is very persistent and even maintained if the orientation of a large fraction neurons is frozen in time. In contradistinction, in maps this procedure leads to a drastic decrease in the changes in orientation of the remaining neurons.

The theory developed explains the mechanism to dynamically generate and maintain interspersed patterns of selectivities. It, however, does not answer the question when each of the two distinct layouts is more advantageous for the organism. To answer this question, a simple optimization theory was constructed and examined that predicts a transition between the two different layout types. Contrary to the previous model, this optimization theory is not dynamical. Here a cost function is constructed and layouts of the different types are tested to find which one minimizes this cost. By varying the size of the model visual cortex using experimentally derived rules (i.e. essentially constant hypercolumn-size in maps [20,27]), the layout type with smaller cost switches from interspersed to maps with increasing area size. The cost function is constructed as a tradeoff between two factors: 1) stimulus coverage, which is improved in interspersed patterns compared to maps but the difference decreases with increasing cortex size and 2) wiring costs per neuron, which is reduced in maps compared to interspersed layouts and keeps constant with area size. Using this cost function, independent of how the different factors are weighted relative to each other, a size dependent transition between the pattern with minimal cost is obtained.

Finally, the prediction of increased lability in interspersed patterns was tested experimentally in the visual cortex of the rat using two-photon calcium imaging. Confidence intervals of the estimated orientation preference of individual neurons are usually not calculated in the common literature. Therefore, the first goal was to find a stimulus and estimation method that reduces the confidence intervals, such that preferred orientation can be measured with maximal precision and the significant changes in time can be detected even if they are small. Episodic stimulation and Bayesian inference of the tuning curve [147] were found to be the best choice to achieve this goal: the estimated orientation shows no significant difference with other conventional fitting methods but the confidence intervals are smaller and could be obtained with fewer presentations of the stimulus. The results show that 
the confidence intervals of the preferred orientation can go down to a few degrees for some neurons, even if estimated from calcium imaging data. In accordance to the predictions of the model, using this fitting method in a recording subdivided in subsections it was found that in less than 30 minutes at least $20 \%$ of the neurons showed significant changes in their orientation preference. The measured shifts range from a few degrees up to an example of a perpendicular change in orientation preference. It is important to keep in mind that the results presented here are preliminary, and experiments explicitly designed to comprehensively test this prediction are being currently performed. In the model the amount of change in orientation depends on the strength of the input noise, where with no noise the dynamics slows down and change takes long. Following this it would be interesting to measure experimentally how different noise levels, represented by the activity of the network the neuron is embedded in, have a repercussion on its orientation lability.

The results of this thesis are obtained by the implementation of a large repertoire of mathematical models and techniques. The thesis starts with constructing an abstract order-parameter model, examines it with analytical methods from nonlinear stability theory and extensive numerical simulations, conclusions are confirmed with a correlation model based on Hebbian learning and finishes with a functional optimization model. Although the order-parameter model is based on the longinteraction model previously defined [21, 22], the analysis approach used here for the first time is very different. While in the previous case weakly-nonlinear analysis was used to formulate the amplitude equations, in the generalized model perturbation theory on a one dimensional system is used. This approach allows to derive an exact equation for the growth and stability of different ordered patterns without the need to specify the interaction function between the neurons (the only condition the interactions have to satisfy are the symmetries assumed in the model). The large set of parameters needed to describe the interaction function are reduced to 3 by using experimental results in rodents and by combining parameters that lead to the same effective equations. This reduced number of parameters allows to test the stability equation analytically for all possible interaction kernels, such that the conclusion that strong local inhibition makes all ordered patterns unstable is robust for all interactions satisfying this condition. To further validate the results large scale simulations of a system of interacting neurons were implemented to calculate the phase diagrams of the model by a completely independent massively computational approach. There is a perfect match between the simulations and the theoretical results up to the limits of the numerical discretization. The expansion of the model to binocular neurons is constructed based solely on symmetry considerations of the inter- and intra-neuron coupling dynamics. Using this a very high dimensional model space that includes all possible interactions up to third order is reduced to 4 intra-neuron and 4 inter-neuron coupling terms, allowing the derivation of an analytical expression for the emergence and stability of different binocularity states. Once again, the results of the theory have a perfect match with extensive simulations. From the mathematical side alone, these calculations reported substantially extend the current state of the art and lay the foundation for future rigorous studies of architecture and dynamics of rodent visual cortex.

\section{The role of inhibition in pattern formation}

An important prediction of this thesis is the role of inhibition in forming the interspersed layout type. The notion that inhibition should shape the functional organization of the columnar cortex is well established. In cats, inhibition was chronically enhanced or reduced during development by injection 
of diazepam or DMCM respectively. The response properties of the neurons did not change, but respectively the size of the ocular dominance columns was locally increased or decreased [18]. The results of this experiment show that inhibition modulates the competition between the neurons and has an impact on their functional organization. Therefore, the prediction of the theory that strong inhibition can completely disorganize the functional layout could be congruent to experimental findings. But is inhibition indeed stronger in rodents than in primates and carnivores?

Quantifying inhibition and comparing it between different species is a difficult task. Independent of the orientation preference layout, in all species around $20 \%$ of the neurons in the visual cortex are inhibitory $[187,188]$, where most of the connections are local $[189,190]$. The difference arises with the high variability of inhibitory cell types [191], with variations even within the same animal order (e.g. between mouse and rat [192]). This has led to efforts to agree on a common nomenclature to facilitate the scientific communication [193]. Another difficulty in comparing the results is variations in the quantification depending on the method applied, which includes electron microscopy, histology, electrophysiology and correlation analysis between others. Since genetic tools are more available in rodents the different inhibitory neuron subtypes can be tested independently with good specificity. Therefore, the body of quantitative results is much higher for interspersed patterns. The values obtained in the different studies are nevertheless somewhat variable and all comparisons have to be taken with a grain of salt. Keeping these caveats in mind, the available experimental data supports the requirements of the model:

- In species with maps, most connections of excitatory neurons are to other excitatory neurons. Using electron microscopy in macaques it was reported that $80 \%$ of interactions are between pyramidal cells (PC) [194] and with the same method in cats that the inhibitory neurons represent only around 5\% of the excitatory post-synaptic targets [195]. Furthermore, using biocytin labeling in cat it was shown that the number of inhibitory boutons connecting to excitatory neuron is 5 times lower than excitatory boutons [76].

- In contrast to this results, in animals with interspersed architecture excitatory neurons are highly connected to inhibitory neurons. Electrophysiological recordings in rats show that the connections between excitatory cells is sparse, with excitatory to inhibitory interactions 10 times more abundant and mostly reciprocal [196]. Two-photon RuBi-Glutamate uncaging in genetically labeled parvalbumin positive inhibitory neurons (PV) show that they interact with almost all PCs in its vicinity, with $71 \%$ of the connections extending to less than $200 \mu \mathrm{m}$ distance [197]. Similar results are obtained in somatostatin expressing inhibitory cells (SOM) using a two-photon photostimulation technique, showing that they connect to almost all PCs in their vicinity with $50 \%$ of the connections of shorter range than $100 \mu \mathrm{m}$ [148]. PV-inhibitory neurons are also strongly connected with each other, where $61 \%$ of them share electrical and $78 \%$ chemical coupling [198]. This strong inhibitory interaction is independent of the preferred orientation of the PCs, as was suggested by electron microscopy in rats [121] and demonstrated with electrical recordings in mouse [120]. It is also insensitive to different sub-networks of preferentially coupled PCs $[114,148]$. The probability of two pyramidal neurons to be directly connected in rats is half as big as having a disynaptic connection with an inhibitory neuron in between [199]. This connectivity pattern is similar in other functionally interspersed cortical areas. For example in the barrel cortex, an excitatory neuron is connected to another excitatory neuron with $17 \%$ probability, with a fast-spiking inhibitory cells with $58 \%$ and with a non-fast-spiking inhibitory cell with $24 \%$ probability [200]. The functional consequence of this is that the activa- 
tion of one excitatory cell increases the recurrent inhibition in the network supralinearly [201], leading to a strong lateral surround suppression in the corresponding cortical layer [149].

Taken together this shows that short-ranged inhibition is indeed stronger in rodents than in species with maps. This fact has functional repercussions on the properties of neurons in the rodent cortex, making them different from species with maps. One clear difference is in the orientation selectivity of inhibitory neurons in both systems. In species with maps inhibitory neurons are sharply tuned for orientation [104, 202, 203]. In contrast, in rodents most studies show that this is not the case [103, 120, 150, 204, 205] (but see [206]). The lack of orientation selectivity in inhibitory neurons was also reported in rabbits [207]. Other studies report that SOM inhibitory neurons are tuned, but that the response is 4 times weaker than PV inhibitory cells [208]. A weak tuning of inhibitory neurons was found to reflect the local bias of preferred orientations of the excitatory cells it is connected to [204]. These observations led to the view that this difference in orientation selectivity between the species arises from the columnar versus interspersed architecture the inhibitory neurons are embedded in. The results obtained in this thesis support a different scenario where the causal link is reversed: Since inhibitory neurons are densely connected to excitatory neurons an interspersed pattern emerges, which is reflected on the selectivity of the inhibitory neurons.

\section{Spatial characterization of the interspersed pattern}

In chapter 4 it was shown that the interspersed pattern generated in the simulations is not random, but has a negative correlation between nearest neighbors. In the simulations the measured correlation is very weak, scaling inversely with the number of interacting neurons, such that any other mechanism not included in the model that lead to an increase in similarity between the receptive fields of neurons can easily overcome its effect. Is this property of the interspersed organization found in the cortex? Experimental studies report a very weak but significant bias of close-by neurons to have a similar orientation preference [100, 102], where the similarity of tuning extends up to $100 \mu \mathrm{m}$ [209]. A similar bias was also reported in the spontaneous activity of the network, where its spatial correlation is slightly higher than random up to $80 \mu \mathrm{m}$ [? ]. As mentioned above, this local bias might underlay the weak orientation preference of the inhibitory neurons that sample from this population [204]. The reported positive correlation doesn't contradict the model. The weak bias might be a remnant of the clonally related neurons that tend to be connected by gap junctions, increasing the similarity of their tuning properties [116, 117]. This gives a biased initial condition that is not incorporated in the model. The same studies also report that this initial similarity in tuning is only maintained early in development and is not present once gap junctions are removed. Similarly, the reported bias should also decrease in time, as neurons dynamically disorganize their tuning properties. Another possible cause is weak orientation selective neuropil contamination induced by some neurons in the LGN that are tuned [91]. This could cause an artifact in the estimation of orientation preferences and falsely lead to a weak local bias.

It is rather surprising that the local bias is very weak and only detectable with statistical methods in spite of the amount of drive to the neurons to develop a similar tuning. In mouse the receptive field size is $10^{\circ}-14^{\circ}$ [103] and the cortical magnification factor $15 \mu \mathrm{m} /{ }^{\circ}$ [98], meaning that the neurons in those $100 \mu \mathrm{m}$ encode a highly overlapping position in visual space, but maintain a highly disordered orientation preference layout. The origin of this local bias could be further understood by analyzing if this averaged orientation of the population is maintained in different cortical depths 
and is therefore columnar, indicating that the radial projections in the cortex are causing this effect [91]. For this approach it is necessary to determine the orientation preference of a population of neurons in a volume of the cortex. The difficulty of such an experiment is in determining the z-axis in the cortex, since it is not necessarily the same as the z-axis of the microscope images and the depth achievable by the microscope doesn't allow to reach clear anatomical cues. In collaboration with Jason Kerr and Vishnudev Ramachandra from the Max Planck Institute for Biological Cybernetics in Tübingen an algorithm was developed to extract the real z-axis in 2-photon calcium imaging in rats by reconstructing the deep radial blood vessels in the cortex from histological slices and matching the coordinates with the functionally imaged volume. With enough data analyzed the results of this experiments will shed a light on causes of this local bias.

\section{Dynamical characterization of the interspersed pattern}

The tendency of the neurons to dynamically increase the orientation difference between them as predicted by the model could be experimentally tested by artificially inducing the formation of an substantial local bias for a similar orientation preference, as shown in the simulations in figure 2.1.1f). This "column" or cluster of similar orientation could be implanted in the cortex by pairing the presentation of the orientation with activity in the neurons by either intra-cortical stimulation or by shining light on genetically encoded cation channels [210,211]. According to the model the imposed order should decrease in time as the column disorganizes. Another experimental test to the dynamical nature of the interspersed layout comes from the binocular model. Binocular neurons show a high degree of change in their tuning to match the orientation preference for inputs of each eye. According to the model the disordered pattern is dynamically maintained, such that monocular neurons in their vicinity would also show a change in their preferred orientation during this process. These experimental procedures test the qualitative prediction that the interspersed pattern is generated dynamically, which is the most important characteristic of the model.

Another dynamical prediction of the model is that neurons in an interspersed architecture have more labile tuning. That this is indeed the case was tested in chapter ?? in the visual cortex of the rat, where a fraction of neurons presented significant changes of orientation preferences even over the time course of an acute experiment of 30 minutes. As stated above this results are preliminary and need to be further confirmed. That the orientation preference of neurons in the rodent cortex can exhibit drastic changes over time has been reported. One example in the visual cortex of this is the binocular matching of orientation preference after the emergence of selectivity [127], or in the barrel cortex where neurons change their direction selectivity long after the classical critical period to increase the degree of functional clustering [133]. In contrast to this, in species with maps the layout of orientation preferences appears much more robust in time [212], where a reorganization coherent with the map structure happens over longer time periods $[172,213]$ unless changes are imposed externally, e.g. by pairing the response of the neurons to a given stimulus [210,211]. The mentioned changes in the tuning of rodents are obtained by measuring different animals of different ages. To elucidate the changes in individual neurons over time chronic imaging experiments have to be used, for example by genetically encoding a calcium sensor in neurons and opening a cranial window for experiments with 2-photon scanning microscopy [214]. This experiments in mice show that the synaptic boutons, and therefore the connection between the neurons, are highly volatile, changing on the time-scale of days and months [215-217]. In some experiments the stability of the 
orientation tuning curve in time has been addressed $[218,219]$, showing that some neurons have a robust preferred orientation over days. The fraction of neurons changing their preferred orientation is not reported as the papers focus on the success of the imaging method. As discussed above, the model predicts that the constancy of the preferred orientation in a fraction of neurons doesn't have a strong impact on the undergoing changes of the other neurons, such that in the experiments both stable and labile tuning curves could be found.

Why would evolution favor a layout where the representation of the neurons is so variable? The first problem that comes to mind is how this labile neurons can be decoded downstream in visual processing. The fact is that it is not known how the information is processed downstream even in species with robust representations and maps. On the positive side, this increased susceptibility to change can support fast adaptation to changing statistics of the visual world. In behavioral experiments where a visual stimulus was associated with a subsequent reward, after 3-7 task repetitions neurons that initially encode the stimulus evolve the ability to also encode the timing of the reward [220]. In rodents visual adaptation has a direct effect on the response of neurons in V1. For example, in mouse the adaptation to a repeated presentation of a grating can be measured as an enhance response evoked by the stimulus in the cortex [221]. Adaptation to repeated stimulation is also present in monkeys, but the effect of the response in the cortex differs. For example in monkeys after visual training of one orientation the neurons only slightly modify their tuning properties, and adaptation is obtained mostly by contextual gain $[222,223]$.

\section{Transition between the layout types}

The optimization model presented in chapter 5 predicts a transition between the different layout types for many examples of connection functions. As shown in the original paper [64], with increasing sharpness of the connection selectivity the layout that minimizes the wiring cost changes from disordered patterns to periodic maps. This means that if the connection selectivity is set to be cosine shaped as found in experiments [64,115], the optimal pattern for wiring costs alone are ordered stripes. In that case both interspersed and periodic maps are not optimal and the difference in wiring cost is reduced, to the point that the benefits are flipped and the transition no longer occurs. The key element of the present model is the area size dependent difference in coverage between the layouts, and the role of the wiring cost is simply that of an area size independent cost component that is reduced in maps compared to interspersed patterns. In any other area size independent cost function that associates benefits with the presence of maps the combined cost function will show the described transition. Possible cost functions with this property could be a penalty for the dynamical lability of the orientations or the enhanced redundancy between the neurons when a stimulus is presented. For any such cost function it is important to keep in mind that the predicted transition is qualitatively robust but quantitatively modifiable. As pointed out by Van Hooser et al. [110] there is an overlap of V1 sizes having different orientation layouts: gray squirrel and ferret have a V1 area size of $\sim 80 \mathrm{~m} \mathrm{~m}^{2}$ and $75-90 \mathrm{~m} \mathrm{~m}^{2}$ respectively, and the first one doesn't have orientation maps and the second one does. To explain this discrepancy, a cost function would have to be constructed that contains other aspects besides the V1 area. It could include for example the fact that the inverse magnification factor is $3.5-4^{\circ} / \mathrm{mm}^{2}$ in the gray squirrel compared to $2.5^{\circ} / \mathrm{mm}^{2}$ in ferret, such that with equal cortex size the fraction activated by $1^{\circ}$ stimulus is smaller in the first compared to the second. In the model, to calculate the coverage the fraction of the cortex activated by the stimulus is constant for both pat- 
terns. Including this difference would therefore induce a shift in the transition boundary even with equal cortex size. Another example of a factor that could lead to different layouts with equal cortex size is the neuronal density, which is smaller in rodents compared to carnivores [138]. This means that although the gray squirrel and the ferret V1 have approximately the same size, the number of neurons is smaller in the first. In the model to calculate the wiring costs the number of connection a neuron has to have is the same for both patterns, and therefore would be even higher in rodents if the neurons are further apart. These two examples show that once a cost function is constructed with a higher degree of detail, this would shift the value of the predicted transition but not its presence. In the model a similar change results from modifying the relative strength of the factors in the cost function. It was shown that independently of the chosen scaling a transition between the layouts types always exists. Instead of focusing on the details of the model for animals of similar cortex size, a better approach to critically test it would be to experimentally examine animals that lay far from the transition boundary, i.e rodents with a large V1 and primates, carnivores or other laurasiatherians with small V1. In the rodent order an animal fulfilling this property would be the capybara or the agouti, with V1 sizes of $300 \mathrm{~m} \mathrm{~m}^{2}$ and $280 \mathrm{~m} \mathrm{~m}^{2}$ respectively [164, 224]. On the primate side the test animal could be the mouse lemur with a V1 size of $10 \mathrm{~m} \mathrm{~m}^{2}$ [173]. For the laurasiatherians a very fascinating case is the etruscan shrew, with a V1 size of $0.3 \mathrm{~mm}^{2}$ [168]. Although it belongs to a mammalian clade where usually orientation maps are reported, the total V1 area is smaller than the typical size of one hypercolumn. This model predicts an interspersed architecture in this species.

Independent of the details that can be added, the theory developed here for the first time makes it transparent how cortex size could drive a transition between layout types. For understanding the rodent condition this model enables us to explain why they lack orientation maps in a quantitatively and precise way and identifies a possible functional advantage associated with its evolutionary emergence or maintenance. 


\section{Bibliography}

[1] R. Llinás. I of the vortex: from neurons to self. MIT, Cambridge, Mass.; London, 2002.

[2] A. Goate, M.-C. Chartier-Harlin, M. Mullan, J. Brown, F. Crawford, L. Fidani, L. Giuffra, A. Haynes, N. Irving, L. James, R. Mant, P. Newton, K. Rooke, P. Roques, C. Talbot, M. PericakVance, A. Roses, R. Williamson, M. Rossor, M. Owen, and J. Hardy. Segregation of a missense mutation in the amyloid precursor protein gene with familial alzheimer's disease. Nature, 349(6311):704-706, Feb. 1991.

[3] F. Chaouloff. Serotonin, stress and corticoids. Journal of Psychopharmacology, 14(2):139-151, Mar. 2000.

[4] W. Gerstner, H. Sprekeler, and G. Deco. Theory and simulation in neuroscience. Science, 338(6103):60-65, Oct. 2012.

[5] G. A. Parker and J. M. Smith. Optimality theory in evolutionary biology. Nature, 348(6296):2733, Nov. 1990.

[6] H. D. Simpson, D. Mortimer, and G. J. Goodhill. Chapter 1 theoretical models of neural circuit development. In Development of Neural Circuitry, volume 87, pages 1-51. Academic Press, 2009.

[7] A. L. Hodgkin and A. F. Huxley. A quantitative description of membrane current and its application to conduction and excitation in nerve. The Journal of Physiology, 117(4):500-544, Aug. 1952.

[8] D. O. Hebb. The organization of behavior; a neuropsychological theory, volume xix. Wiley, Oxford, England, 1949.

[9] E. Neher, B. Sakmann, and J. H. Steinbach. The extracellular patch clamp: a method for resolving currents through individual open channels in biological membranes. Pflügers Archiv: European journal of physiology, 375(2):219-228, July 1978.

[10] E. R. Kandel and J. H. Schwartz. Molecular biology of learning: modulation of transmitter release. Science, 218(4571):433-443, Oct. 1982.

[11] G. J. Goodhill. Contributions of theoretical modeling to the understanding of neural map development. Neuron, 56(2):301-311, Oct. 2007.

[12] N. V. Swindale, J. A. Matsubara, and M. S. Cynader. Surface organization of orientation and direction selectivity in cat area 18. The Journal of neuroscience: the official journal of the Society for Neuroscience, 7(5):1414-1427, May 1987. 
[13] G. G. Blasdel and G. Salama. Voltage-sensitive dyes reveal a modular organization in monkey striate cortex. Nature, 321(6070):579-585, June 1986.

[14] T. Bonhoeffer and A. Grinvald. Iso-orientation domains in cat visual cortex are arranged in pinwheel-like patterns. Nature, 353(6343):429-431, Oct. 1991.

[15] G. J. Goodhill. Topography and ocular dominance: a model exploring positive correlations. Biological cybernetics, 69(2):109-118, 1993.

[16] K. D. Miller, J. B. Keller, and M. P. Stryker. Ocular dominance column development: analysis and simulation. Science (New York, N.Y.), 245(4918):605-615, Aug. 1989.

[17] S. Löwel. Ocular dominance column development: strabismus changes the spacing of adjacent columns in cat visual cortex. The Journal of neuroscience: the official journal of the Society for Neuroscience, 14(12):7451-7468, Dec. 1994.

[18] T. K. Hensch and M. P. Stryker. Columnar architecture sculpted by GABA circuits in developing cat visual cortex. Science, 303(5664):1678-1681, Mar. 2004.

[19] M. Kaschube, M. Schnabel, and F. Wolf. Self-organization and the selection of pinwheel density in visual cortical development. New Journal of Physics, 10(1):015009, Jan. 2008.

[20] M. Kaschube, M. Schnabel, S. Löwel, D. M. Coppola, L. E. White, and F. Wolf. Universality in the evolution of orientation columns in the visual cortex. Science, 330(6007):1113-1116, Nov. 2010.

[21] F. Wolf. Symmetry, multistability, and long-range interactions in brain development. Physical Review Letters, 95(20):208701, Nov. 2005.

[22] F. Wolf and T. Geisel. Spontaneous pinwheel annihilation during visual development. Nature, 395(6697):73-78, 1998.

[23] D. H. Hubel and T. N. Wiesel. Ferrier lecture. functional architecture of macaque monkey visual cortex. Proceedings of the Royal Society of London. Series B, Biological Sciences., 198(1130):1-59, July 1977.

[24] M. Hübener, D. Shoham, A. Grinvald, and T. Bonhoeffer. Spatial relationships among three columnar systems in cat area 17. The Journal of Neuroscience, 17(23):9270-9284, Dec. 1997.

[25] D. J. Felleman and J. H. Kaas. Receptive-field properties of neurons in middle temporal visual area (MT) of owl monkeys. Journal of Neurophysiology, 52(3):488-513, Sept. 1984.

[26] K. Tanaka, H. Saito, Y. Fukada, and M. Moriya. Coding visual images of objects in the inferotemporal cortex of the macaque monkey. Journal of Neurophysiology, 66(1):170-189, July 1991.

[27] W. Keil, M. Kaschube, M. Schnabel, Z. F. Kisvarday, S. Löwel, D. M. Coppola, L. E. White, and F. Wolf. Response to comment on "Universality in the evolution of orientation columns in the visual cortex". Science, 336(6080):413-413, Apr. 2012.

[28] D. H. Hubel and T. N. Wiesel. Receptive fields of single neurones in the cat's striate cortex. The Journal of Physiology, 148(3):574-591, Oct. 1959. 
[29] L. E. White, D. M. Coppola, and D. Fitzpatrick. The contribution of sensory experience to the maturation of orientation selectivity in ferret visual cortex. Nature, 411(6841):1049-1052, June 2001.

[30] M. Weliky and L. C. Katz. Functional mapping of horizontal connections in developing ferret visual cortex: experiments and modeling. The Journal of Neuroscience, 14(12):7291-7305, Dec. 1994.

[31] B. Chapman and M. P. Stryker. Development of orientation selectivity in ferret visual cortex and effects of deprivation. The Journal of Neuroscience, 13(12):5251-5262, Dec. 1993.

[32] P. G. Clarke and D. Whitteridge. The cortical visual areas of the sheep. The Journal of Physiology, 256(3):497-508, Apr. 1976.

[33] R. Malach, R. B. H. Tootell, and D. Malonek. Relationship between orientation domains, cytochrome oxidase stripes, and intrinsic horizontal connections in squirrel monkey area v2. Cerebral Cortex, 4(2):151-165, Mar. 1994.

[34] K. Obermayer and G. G. Blasdel. Singularities in primate orientation maps. Neural Computation, 9(3):555-575, Mar. 1997.

[35] D. H. Hubel, T. N. Wiesel, and M. P. Stryker. Anatomical demonstration of orientation columns in macaque monkey. The Journal of Comparative Neurology, 177(3):361-379, 1978.

[36] X. Xu, W. Bosking, G. Sáry, J. Stefansic, D. Shima, and V. Casagrande. Functional organization of visual cortex in the owl monkey. The Journal of Neuroscience, 24(28):6237-6247, July 2004.

[37] X. Xu, W. H. Bosking, L. E. White, D. Fitzpatrick, and V. A. Casagrande. Functional organization of visual cortex in the prosimian bush baby revealed by optical imaging of intrinsic signals. Journal of Neurophysiology, 94(4):2748-2762, Oct. 2005.

[38] E. Yacoub, N. Harel, and K. Uğurbil. High-field fMRI unveils orientation columns in humans. Proceedings of the National Academy of Sciences, 105(30):10607-10612, July 2008.

[39] W. H. Bosking, Y. Zhang, B. Schofield, and D. Fitzpatrick. Orientation selectivity and the arrangement of horizontal connections in tree shrew striate cortex. The Journal of Neuroscience, 17(6):2112-2127, Mar. 1997.

[40] A. Grinvald, E. Lieke, R. D. Frostig, C. D. Gilbert, and T. N. Wiesel. Functional architecture of cortex revealed by optical imaging of intrinsic signals. Nature, 324(6095):361-364, Nov. 1986.

[41] L. Reichl, D. Heide, S. Löwel, J. C. Crowley, M. Kaschube, and F. Wolf. Coordinated optimization of visual cortical maps (i) symmetry-based analysis. PLoS Computational Biology, 8(11):e1002466, Nov. 2012.

[42] L. Reichl, D. Heide, S. Löwel, J. C. Crowley, M. Kaschube, and F. Wolf. Coordinated optimization of visual cortical maps (II) numerical studies. PLoS Computational Biology, 8(11):e1002756, Nov. 2012.

[43] D. H. Hubel and T. N. Wiesel. Shape and arrangement of columns in cat's striate cortex. The Journal of Physiology, 165(3):559-568.2, Mar. 1963. 
[44] D. Ferster, S. Chung, and H. Wheat. Orientation selectivity of thalamic input to simple cells of cat visual cortex. Nature, 380(6571):249-252, Mar. 1996.

[45] M. C. Crair, D. C. Gillespie, and M. P. Stryker. The role of visual experience in the development of columns in cat visual cortex. Science, 279(5350):566-570, Jan. 1998.

[46] G. Goodhill and D. Willshaw. Application of the elastic net algorithm to the formation of ocular dominance stripes. Network: Computation in Neural Systems, 1(1):41-59, 1990.

[47] C. v. d. Malsburg. Self-organization of orientation sensitive cells in the striate cortex. Kybernetik, 14(2):85-100, June 1973.

[48] N. V. Swindale and H. Bauer. Application of kohonen's self-organizing feature map algorithm to cortical maps of orientation and direction preference. Proceedings of the Royal Society of London. Series B: Biological Sciences, 265(1398):827-838, May 1998.

[49] N. V. Swindale. The development of topography in the visual cortex: a review of models. Network (Bristol, England), 7(2):161-247, May 1996.

[50] K. Kang, M. Shelley, and H. Sompolinsky. Mexican hats and pinwheels in visual cortex. Proceedings of the National Academy of Sciences of the United States of America, 100(5):2848 -2853, Mar. 2003.

[51] C. v. d. Malsburg. Development of ocularity domains and growth behaviour of axon terminals. Biological Cybernetics, 32(1):49-62, Mar. 1979.

[52] R. Linsker. From basic network principles to neural architecture: emergence of spatialopponent cells. Proceedings of the National Academy of Sciences, 83(19):7508-7512, Oct. 1986.

[53] K. D. Miller. A model for the development of simple cell receptive fields and the ordered arrangement of orientation columns through activity-dependent competition between $\mathrm{ON}$ - and OFF-center inputs. The Journal of Neuroscience: The Official Journal of the Society for Neuroscience, 14(1):409-441, Jan. 1994.

[54] A. Grabska-Barwińska and C. v. d. Malsburg. Establishment of a scaffold for orientation maps in primary visual cortex of higher mammals. The Journal of Neuroscience, 28(1):249-257, Jan. 2008.

[55] R. Durbin and G. Mitchison. A dimension reduction framework for understanding cortical maps. Nature, 343(6259):644-647, Feb. 1990.

[56] N. V. Swindale, D. Shoham, A. Grinvald, T. Bonhoeffer, and M. Hubener. Visual cortex maps are optimized for uniform coverage. Nature Neuroscience, 3(8):822-826, 2000.

[57] M. á. Carreira-Perpiñán and G. J. Goodhill. Are visual cortex maps optimized for coverage? Neural Computation, 14(7):1545-1560, July 2002.

[58] C. E. Giacomantonio, M. R. Ibbotson, and G. J. Goodhill. The influence of restricted orientation rearing on map structure in primary visual cortex. NeuroImage, 52(3):875-883, Sept. 2010. 
[59] C. E. Giacomantonio and G. J. Goodhill. The effect of angioscotomas on map structure in primary visual cortex. The Journal of Neuroscience, 27(18):4935-4946, May 2007.

[60] W. Keil and F. Wolf. Coverage, continuity, and visual cortical architecture. Neural Systems $\mathcal{E}$ Circuits, 1(1):1-56, Dec. 2011.

[61] O. Scherf, K. Pawelzik, F. Wolf, and T. Geisel. Theory of ocular dominance pattern formation. Physical Review E, 59(6):6977-6993, June 1999.

[62] T. Kohonen. Self-organized formation of topologically correct feature maps. Biological Cybernetics, 43(1):59-69, Jan. 1982.

[63] T. Kohonen. Self-Organizing Maps, volume 30. Springer, 2001.

[64] A. A. Koulakov and D. B. Chklovskii. Orientation preference patterns in mammalian visual cortex: a wire length minimization approach. Neuron, 29(2):519-527, Feb. 2001.

[65] D. B. Chklovskii and A. A. Koulakov. Maps in the brain: What can we learn from them? Annual Review of Neuroscience, 27(1):369-392, July 2004.

[66] N. V. Swindale. A model for the formation of orientation columns. Proceedings of the Royal Society of London. Series B, Biological Sciences, 215(1199):211-230, May 1982.

[67] L. Reichl, S. Lï œwel, and F. Wolf. Pinwheel stabilization by ocular dominance segregation. Physical Review Letters, 102(20):208101, May 2009.

[68] P. C. Bressloff, J. D. Cowan, M. Golubitsky, and P. J. Thomas. Scalar and pseudoscalar bifurcations motivated by pattern formation on the visual cortex. Nonlinearity, 14(4):739, July 2001.

[69] P. C. Bressloff and J. D. Cowan. The visual cortex as a crystal. Physica D: Nonlinear Phenomena, 173(3-4):226-258, Dec. 2002.

[70] P. J. Thomas and J. D. Cowan. Simultaneous constraints on pre- and post-synaptic cells couple cortical feature maps in a 2D geometric model of orientation preference. Mathematical Medicine and Biology, 23(2):119-138, June 2006.

[71] P. J. Thomas and J. D. Cowan. Symmetry induced coupling of cortical feature maps. Physical Review Letters, 92(18):188101, May 2004.

[72] H. Y. Lee, M. Yahyanejad, and M. Kardar. Symmetry considerations and development of pinwheels in visual maps. Proceedings of the National Academy of Sciences, 100(26):16036-16040, Dec. 2003.

[73] F. Sengpiel, P. Stawinski, and T. Bonhoeffer. Influence of experience on orientation maps in cat visual cortex. Nature Neuroscience, 2(8):727-732, Aug. 1999.

[74] J. Swift and P. C. Hohenberg. Hydrodynamic fluctuations at the convective instability. Physical Review A, 15(1):319-328, Jan. 1977.

[75] M. Cross and H. Greenside. Pattern Formation and Dynamics in Nonequilibrium Systems. Cambridge University Press, Cambridge, 2009. 
[76] Z. F. Kisvárday, E. Tóth, M. Rausch, and U. T. Eysel. Orientation-specific relationship between populations of excitatory and inhibitory lateral connections in the visual cortex of the cat. Cerebral Cortex, 7(7):605-618, Oct. 1997.

[77] L. E. White, D. M. Coppola, and D. Fitzpatrick. The contribution of sensory experience to the maturation of orientation selectivity in ferret visual cortex. Nature, 411(6841):1049-1052, June 2001.

[78] K. E. Schmidt, R. Goebel, S. Löwel, and W. Singer. The perceptual grouping criterion of colinearity is reflected by anisotropies of connections in the primary visual cortex. European Journal of Neuroscience, 9(5):1083-1089, 1997.

[79] H. J. Chisum, F. Mooser, and D. Fitzpatrick. Emergent properties of layer 2/3 neurons reflect the collinear arrangement of horizontal connections in tree shrew visual cortex. The Journal of Neuroscience, 23(7):2947-2960, Apr. 2003.

[80] S. D. Van Hooser. Similarity and diversity in visual cortex: Is there a unifying theory of cortical computation? Neuroscientist, 13(6):639-656, Dec. 2007.

[81] N. Cristopher M. Exploring the next frontier of mouse vision. Neuron, 72(6):889-892, Dec. 2011.

[82] G. T. Prusky, P. W. West, and R. M. Douglas. Behavioral assessment of visual acuity in mice and rats. Vision Research, 40(16):2201-2209, 2000.

[83] J. B. Calderone and G. H. Jacobs. Cone receptor variations and their functional consequences in two species of hamster. Visual Neuroscience, 16(01):53-63, 1999.

[84] G. T. Prusky, P. W. West, and R. M. Douglas. Behavioral assessment of visual acuity in mice and rats. Vision Research, 40(16):2201-2209, July 2000.

[85] D. J. Wallace, D. S. Greenberg, J. Sawinski, S. Rulla, G. Notaro, and J. N. D. Kerr. Rats maintain an overhead binocular field at the expense of constant fusion. Nature, 498(7452):65-69, June 2013.

[86] B. Völgyi, S. Chheda, and S. A. Bloomfield. Tracer coupling patterns of the ganglion cell subtypes in the mouse retina. The Journal of Comparative Neurology, 512(5):664-687, 2009.

[87] M. Rivlin-Etzion, K. Zhou, W. Wei, J. Elstrott, P. L. Nguyen, B. A. Barres, A. D. Huberman, and M. B. Feller. Transgenic mice reveal unexpected diversity of on-off direction-selective retinal ganglion cell subtypes and brain structures involved in motion processing. The Journal of Neuroscience, 31(24):8760-8769, June 2011.

[88] T. A. Münch, R. A. da Silveira, S. Siegert, T. J. Viney, G. B. Awatramani, and B. Roska. Approach sensitivity in the retina processed by a multifunctional neural circuit. Nature neuroscience, 12(10):1308-1316, Oct. 2009.

[89] U. C. Dräger and J. F. Olsen. Origins of crossed and uncrossed retinal projections in pigmented and albino mice. The Journal of comparative neurology, 191(3):383-412, June 1980.

[90] C. Ling, G. E. Schneider, and S. Jhaveri. Target-specific morphology of retinal axon arbors in the adult hamster. Visual neuroscience, 15(3):559-579, June 1998. 
[91] B. Scholl, A. Y. Y. Tan, J. Corey, and N. J. Priebe. Emergence of orientation selectivity in the mammalian visual pathway. The Journal of Neuroscience, 33(26):10616-10624, June 2013.

[92] D. M. Piscopo, R. N. El-Danaf, A. D. Huberman, and C. M. Niell. Diverse visual features encoded in mouse lateral geniculate nucleus. The Journal of Neuroscience, 33(11):4642-4656, Mar. 2013.

[93] M. S. Grubb and I. D. Thompson. Quantitative characterization of visual response properties in the mouse dorsal lateral geniculate nucleus. Journal of neurophysiology, 90(6):3594-3607, Dec. 2003.

[94] X. Zhao, H. Chen, X. Liu, and J. CANG. Orientation-selective responses in the mouse lateral geniculate nucleus. The Journal of Neuroscience, 33(31):12751-12763, July 2013.

[95] K. G. Thompson, A. G. Leventhal, Y. Zhou, and D. Liu. Stimulus dependence of orientation and direction sensitivity of cat LGNd relay cells without cortical inputs: a comparison with area 17 cells. Visual neuroscience, 11(5):939-951, Oct. 1994.

[96] W. R. Levick, C. W. Oyster, and E. Takahashi. Rabbit lateral geniculate nucleus: sharpener of directional information. Science (New York, N.Y.), 165(3894):712-714, Aug. 1969.

[97] S. K. Cheong, C. Tailby, S. G. Solomon, and P. R. Martin. Cortical-like receptive fields in the lateral geniculate nucleus of marmoset monkeys. The Journal of Neuroscience, 33(16):6864-6876, Apr. 2013.

[98] S. Schuett, T. Bonhoeffer, and M. Hubener. Mapping retinotopic structure in mouse visual cortex with optical imaging. The Journal of Neuroscience, 22(15):6549-6559, Aug. 2002.

[99] C. M. Niell and M. P. Stryker. Modulation of visual responses by behavioral state in mouse visual cortex. Neuron, 65(4):472-479, Feb. 2010.

[100] K. Ohki, S. Chung, Y. H. Ch'ng, P. Kara, and R. C. Reid. Functional imaging with cellular resolution reveals precise micro-architecture in visual cortex. Nature, 433(7026):597-603, Feb. 2005.

[101] S. L. Smith and M. Hausser. Parallel processing of visual space by neighboring neurons in mouse visual cortex. Nature Neuroscience, 13(9):1144-1149, Aug. 2010.

[102] U. C. Dräger. Receptive fields of single cells and topography in mouse visual cortex. The Journal of comparative neurology, 160(3):269-290, Apr. 1975.

[103] C. M. Niell and M. P. Stryker. Highly selective receptive fields in mouse visual cortex. The Journal of Neuroscience, 28(30):7520-7536, July 2008.

[104] J. S. Anderson, M. Carandini, and D. Ferster. Orientation tuning of input conductance, excitation, and inhibition in cat primary visual cortex. Journal of Neurophysiology, 84(2):909-926, Aug. 2000 .

[105] N. J. Mangini and A. L. Pearlman. Laminar distribution of receptive field properties in the primary visual cortex of the mouse. The Journal of comparative neurology, 193(1):203-222, Sept. 1980. 
[106] C. Métin, P. Godement, and M. Imbert. The primary visual cortex in the mouse: Receptive field properties and functional organization. Experimental Brain Research, 69(3):594-612, Feb. 1988.

[107] S. V. Girman, Y. Sauvé, and R. D. Lund. Receptive field properties of single neurons in rat primary visual cortex. Journal of Neurophysiology, 82(1):301 -311, July 1999.

[108] M. Fagiolini, T. Pizzorusso, N. Berardi, L. Domenici, and L. Maffei. Functional postnatal development of the rat primary visual cortex and the role of visual experience: Dark rearing and monocular deprivation. Vision Research, 34(6):709-720, Mar. 1994.

[109] T. Y-C and C. Blakemore. Functional organization in the superior colliculus of the golden hamster. The Journal of Comparative Neurology, 168(4):483-503, Aug. 1976.

[110] S. D. Van Hooser, J. A. F. Heimel, S. Chung, S. B. Nelson, and L. J. Toth. Orientation selectivity without orientation maps in visual cortex of a highly visual mammal. The Journal of Neuroscience: The Official Journal of the Society for Neuroscience, 25(1):19-28, Jan. 2005.

[111] E. H. Murphy and N. Berman. The rabbit and the cat: A comparison of some features of response properties of single cells in the primary visual cortex. The Journal of Comparative Neurology, 188(3):401-427, Dec. 1979.

[112] K. Ohki and R. C. Reid. Specificity and randomness in the visual cortex. Current Opinion in Neurobiology, 17(4):401-407, Aug. 2007.

[113] S. Song, P. J. Sjöström, M. Reigl, S. Nelson, and D. B. Chklovskii. Highly nonrandom features of synaptic connectivity in local cortical circuits. PLoS Biology, 3(3):e68, Mar. 2005.

[114] Y. Yoshimura, J. L. M. Dantzker, and E. M. Callaway. Excitatory cortical neurons form fine-scale functional networks. Nature, 433(7028):868-873, Feb. 2005.

[115] H. Ko, S. B. Hofer, B. Pichler, K. A. Buchanan, P. J. Sjostrom, and T. D. Mrsic-Flogel. Functional specificity of local synaptic connections in neocortical networks. Nature, 473(7345):87-91, May 2011.

[116] G. Ohtsuki, M. Nishiyama, T. Yoshida, T. Murakami, M. Histed, C. Lois, and K. Ohki. Similarity of visual selectivity among clonally related neurons in visual cortex. Neuron, 75(1):65-72, July 2012.

[117] Y. Li, H. Lu, P.-1. Cheng, S. Ge, H. Xu, S.-H. Shi, and Y. Dan. Clonally related visual cortical neurons show similar stimulus feature selectivity. Nature, 486(7401):118-121, May 2012.

[118] Y.-C. Yu, R. S. Bultje, X. Wang, and S.-H. Shi. Specific synapses develop preferentially among sister excitatory neurons in the neocortex. Nature, 458(7237):501-504, Mar. 2009.

[119] Y. Yoshimura and E. M. Callaway. Fine-scale specificity of cortical networks depends on inhibitory cell type and connectivity. Nat Neurosci, 8(11):1552-1559, Nov. 2005.

[120] S. B. Hofer, H. Ko, B. Pichler, J. Vogelstein, H. Ros, H. Zeng, E. Lein, N. A. Lesica, and T. D. Mrsic-Flogel. Differential connectivity and response dynamics of excitatory and inhibitory neurons in visual cortex. Nature Neuroscience, 14(8):1045-1052, Aug. 2011. 
[121] D. D. Bock, W.-C. A. Lee, A. M. Kerlin, M. L. Andermann, G. Hood, A. W. Wetzel, S. Yurgenson, E. R. Soucy, H. S. Kim, and R. C. Reid. Network anatomy and in vivo physiology of visual cortical neurons. Nature, 471(7337):177-182, Mar. 2011.

[122] L. E. White, W. H. Bosking, and D. Fitzpatrick. Consistent mapping of orientation preference across irregular functional domains in ferret visual cortex. Visual Neuroscience, 18(01):65-76, 2001.

[123] S. D. Van Hooser, J. A. Heimel, S. Chung, and S. B. Nelson. Lack of patchy horizontal connectivity in primary visual cortex of a mammal without orientation maps. J. Neurosci., 26(29):76807692, July 2006.

[124] H. Ko, L. Cossell, C. Baragli, J. Antolik, C. Clopath, S. B. Hofer, and T. D. Mrsic-Flogel. The emergence of functional microcircuits in visual cortex. Nature, 496(7443):96-100, Apr. 2013.

[125] H. Jia, N. L. Rochefort, X. Chen, and A. Konnerth. Dendritic organization of sensory input to cortical neurons in vivo. Nature, 464(7293):1307-1312, Apr. 2010.

[126] N. J. Priebe and D. Ferster. Neuroscience: Each synapse to its own. Nature, 464(7293):1290-1291, Apr. 2010.

[127] B.-S. Wang, R. Sarnaik, and J. Cang. Critical period plasticity matches binocular orientation preference in the visual cortex. Neuron, 65(2):246-256, Jan. 2010.

[128] D. Hansel and C. v. Vreeswijk. The mechanism of orientation selectivity in primary visual cortex without a functional map. The Journal of Neuroscience, 32(12):4049-4064, Mar. 2012.

[129] T. A. Woolsey and H. Van der Loos. The structural organization of layer IV in the somatosensory region (SI) of mouse cerebral cortex. the description of a cortical field composed of discrete cytoarchitectonic units. Brain research, 17(2):205-242, Jan. 1970.

[130] M. L. Andermann and C. I. Moore. A somatotopic map of vibrissa motion direction within a barrel column. Nature neuroscience, 9(4):543-551, Apr. 2006.

[131] J. N. D. Kerr, C. P. J. d. Kock, D. S. Greenberg, R. M. Bruno, B. Sakmann, and F. Helmchen. Spatial organization of neuronal population responses in layer $2 / 3$ of rat barrel cortex. The Journal of Neuroscience, 27(48):13316-13328, Nov. 2007.

[132] T. R. Sato, N. W. Gray, Z. F. Mainen, and K. Svoboda. The functional microarchitecture of the mouse barrel cortex. PLoS Biology, 5(7):e189, July 2007.

[133] Y. Kremer, J.-F. Léger, D. Goodman, R. Brette, and L. Bourdieu. Late emergence of the vibrissa direction selectivity map in the rat barrel cortex. The Journal of Neuroscience, 31(29):10689-10700, July 2011.

[134] G. Rothschild, I. Nelken, and A. Mizrahi. Functional organization and population dynamics in the mouse primary auditory cortex. Nature Neuroscience, 13(3):353-360, Mar. 2010.

[135] S. Bandyopadhyay, S. A. Shamma, and P. O. Kanold. Dichotomy of functional organization in the mouse auditory cortex. Nature Neuroscience, 13(3):361-368, Mar. 2010. 
[136] B. Haider, M. Hausser, and M. Carandini. Inhibition dominates sensory responses in the awake cortex. Nature, 2012.

[137] J. J. Hutsler, D.-G. Lee, and K. K. Porter. Comparative analysis of cortical layering and supragranular layer enlargement in rodent carnivore and primate species. Brain research, 1052(1):7181, Aug. 2005.

[138] S. Herculano-Houzel, B. Mota, and R. Lent. Cellular scaling rules for rodent brains. Proceedings of the National Academy of Sciences, 103(32):12138-12143, Aug. 2006.

[139] N. Rochefort, M. Narushima, C. Grienberger, N. Marandi, D. Hill, and A. Konnerth. Development of direction selectivity in mouse cortical neurons. Neuron, 71(3):425-432, Aug. 2011.

[140] Y. Li, D. Fitzpatrick, and L. E. White. The development of direction selectivity in ferret visual cortex requires early visual experience. Nature Neuroscience, 9(5):676-681, May 2006.

[141] S.-B. Paik and D. L. Ringach. Link between orientation and retinotopic maps in primary visual cortex. Proceedings of the National Academy of Sciences, 109(18):7091-7096, May 2012.

[142] S.-B. Paik and D. L. Ringach. Retinal origin of orientation maps in visual cortex. Nature Neuroscience, 14(7):919-925, July 2011.

[143] V. R. A. Hore, J. B. Troy, and S. J. Eglen. Parasol cell mosaics are unlikely to drive the formation of structured orientation maps in primary visual cortex. Visual neuroscience, 29(6):283-299, Nov. 2012.

[144] M. Schottdorf, W. Keil, M. Schnabel, D. M. Coppola, S. Löwel, L. E. White, M. Kaschube, and F. Wolf. Do orientation preference maps arise from hexagonal retinal ganglion cell mosaics? In CoSyNe13, Salt Lake City, Utah, USA, Feb. 2013.

[145] W. Keil. Optimization principles and constraints shaping visual cortical architecture. PhD thesis, University of Göttingen, Department of Physics, Göttingen, 2012.

[146] V. A. Kalatsky and M. P. Stryker. New paradigm for optical imaging: temporally encoded maps of intrinsic signal. Neuron, 38(4):529-545, May 2003.

[147] B. Cronin, I. H. Stevenson, M. Sur, and K. P. Körding. Hierarchical bayesian modeling and markov chain monte carlo sampling for tuning-curve analysis. Journal of Neurophysiology, 103(1):591-602, Jan. 2010.

[148] E. Fino and R. Yuste. Dense inhibitory connectivity in neocortex. Neuron, 69(6):1188-1203, Mar. 2011.

[149] H. Adesnik and M. Scanziani. Lateral competition for cortical space by layer-specific horizontal circuits. Nature, 464(7292):1155-1160, Apr. 2010.

[150] B.-h. Liu, P. Li, Y. J. Sun, Y.-t. Li, L. I. Zhang, and H. W. Tao. Intervening inhibition underlies simple-cell receptive field structure in visual cortex. Nature Neuroscience, 13(1):89-96, Jan. 2010.

[151] E. Kim and M. Sheng. PDZ domain proteins of synapses. Nature Reviews Neuroscience, 5(10):771-781, Oct. 2004. 
[152] X. Huang, K.-F. Schmidt, B. Goetze, L. Siegrid, and O. Schlüter. PSD-95 lacking synapses in the visual cortex retain a high degree of AMPA receptor silence. In Göttingen Meeting of the German Neuroscience Society, Göttingen, Lower Saxony, Germany, Mar. 2013.

[153] M. Fagiolini, H. Katagiri, H. Miyamoto, H. Mori, S. G. N. Grant, M. Mishina, and T. K. Hensch. Separable features of visual cortical plasticity revealed by n-methyl-d-aspartate receptor $2 \mathrm{~A}$ signaling. Proceedings of the National Academy of Sciences, 100(5):2854-2859, Mar. 2003.

[154] B. Goetze, K.-F. Schmidt, X. Huang, O. Schlüter, and S. Löwel. PSD-95 KO mice retain a juvenile ocular dominance plasticity into late adulthood. In Göttingen Meeting of the German Neuroscience Society, Göttingen, Lower Saxony, Germany, Mar. 2013.

[155] R. Honeycutt. Stochastic runge-kutta algorithms. II. colored noise. Physical Review A, 45(2):604610, Jan. 1992.

[156] R. Honeycutt. Stochastic runge-kutta algorithms. i. white noise. Physical Review A, 45(2):600603, Jan. 1992.

[157] N. Metropolis and S. Ulam. The monte carlo method. Journal of the American Statistical Association, 44(247):335-341, Sept. 1949.

[158] N. V. Swindale. Coverage and the design of striate cortex. Biological Cybernetics, 65(6):415-424, 1991.

[159] D. J. Heeger. Normalization of cell responses in cat striate cortex. Visual neuroscience, 9(2):181197, Aug. 1992.

[160] M. A. O'Leary, J. I. Bloch, J. J. Flynn, T. J. Gaudin, A. Giallombardo, N. P. Giannini, S. L. Goldberg, B. P. Kraatz, Z.-X. Luo, J. Meng, X. Ni, M. J. Novacek, F. A. Perini, Z. S. Randall, G. W. Rougier, E. J. Sargis, M. T. Silcox, N. B. Simmons, M. Spaulding, P. M. Velazco, M. Weksler, J. R. Wible, and A. L. Cirranello. The placental mammal ancestor and the post-k-pg radiation of placentals. Science, 339(6120):662-667, Feb. 2013.

[161] P. Wong and J. H. Kaas. Architectonic subdivisions of neocortex in the galago (otolemur garnetti). Anatomical record, 293(6):1033-1069, June 2010.

[162] W. C. Hall, J. H. Kaas, H. Killackey, and I. T. Diamond. Cortical visual areas in the grey squirrel (sciurus carolinesis): a correlation between cortical evoked potential maps and architectonic subdivisions. Journal of neurophysiology, 34(3):437-452, May 1971.

[163] H. Holländer and W. Hälbig. Topography of retinal representation in the rabbit cortex: an experimental study using transneuronal and retrograde tracing techniques. The Journal of comparative neurology, 193(3):701-710, Oct. 1980.

[164] G. Campos and W. Welker. Comparisons between brains of a large and a small hystricomorph rodent: Capybara, hydrochoerus and guinea pig, Cavia;Neocortical projection regions and measurements of brain subdivisions. Brain, Behavior and Evolution, 13(4):243-266, 1976.

[165] U. Drenhaus, G. Rager, P. Eggli, and R. Kretz. On the postnatal development of the striate cortex (v1) in the tree shrew (tupaia belangeri). European Journal of Neuroscience, 24(2):479-490, 2006. 
[166] M. I. Law, K. R. Zahs, and M. P. Stryker. Organization of primary visual cortex (area 17) in the ferret. The Journal of comparative neurology, 278(2):157-180, Dec. 1988.

[167] R. J. Tusa, L. A. Palmer, and A. C. Rosenquist. The retinotopic organization of area 17 (striate cortex) in the cat. The Journal of comparative neurology, 177(2):213-235, Jan. 1978.

[168] R. Naumann, F. Anjum, C. Roth-Alpermann, and M. Brecht. Cytoarchitecture, areas, and neuron numbers of the etruscan shrew cortex. The Journal of Comparative Neurology, 520(11):25122530, Aug. 2012.

[169] R. B. Tootell, M. S. Silverman, E. Switkes, and R. L. De Valois. Deoxyglucose analysis of retinotopic organization in primate striate cortex. Science, 218(4575):902-904, Nov. 1982.

[170] S. G. Espinoza and H. C. Thomas. Retinotopic organization of striate and extrastriate visual cortex in the hooded rat. Brain research, 272(1):137-144, Aug. 1983.

[171] N. M. Bugbee and P. S. Goldman-Rakic. Columnar organization of corticocortical projections in squirrel and rhesus monkeys: similarity of column width in species differing in cortical volume. The Journal of comparative neurology, 220(3):355-364, Nov. 1983.

[172] W. Keil, K.-F. Schmidt, S. Löwel, and M. Kaschube. Reorganization of columnar architecture in the growing visual cortex. Proceedings of the National Academy of Sciences, 107(27):12293-12298, July 2010.

[173] H. M. Cooper, H. Kennedy, M. Magnin, and F. Vital-Durand. Thalamic projections to area 17 in a prosimian primate, microcebus murinus. The Journal of comparative neurology, 187(1):145-167, Sept. 1979.

[174] A. Nimmerjahn, F. Kirchhoff, J. N. D. Kerr, and F. Helmchen. Sulforhodamine 101 as a specific marker of astroglia in the neocortex in vivo. Nature Methods, 1(1):31-37, Oct. 2004.

[175] D. H. Brainard. The psychophysics toolbox. Spatial Vision, 10(4):433-436, 1997.

[176] D. G. Pelli. The VideoToolbox software for visual psychophysics: transforming numbers into movies. Spatial vision, 10(4):437-442, 1997.

[177] M. Kleiner, D. Brainard, and D. Pelli. What's new in psychtoolbox-3? Perception, 36(14), 2007.

[178] D. S. Greenberg, A. R. Houweling, and J. N. D. Kerr. Population imaging of ongoing neuronal activity in the visual cortex of awake rats. Nature Neuroscience, 11(7):749-751, July 2008.

[179] J. N. D. Kerr, D. Greenberg, and F. Helmchen. Imaging input and output of neocortical networks in vivo. Proceedings of the National Academy of Sciences of the United States of America, 102(39):14063-14068, Sept. 2005.

[180] B. Efron. Bootstrap methods: Another look at the jackknife. The Annals of Statistics, 7(1):1-26, Jan. 1979.

[181] D. W. Marquardt. An algorithm for least-squares estimation of nonlinear parameters. Journal of the Society for Industrial and Applied Mathematics, 11(2):431-441, June 1963. 
[182] B. Chapman, M. P. Stryker, and T. Bonhoeffer. Development of orientation preference maps in ferret primary visual cortex. The Journal of Neuroscience, 16(20):6443-6453, Oct. 1996.

[183] Y. Frégnac, D. Shulz, S. Thorpe, and E. Bienenstock. A cellular analogue of visual cortical plasticity. Nature, 333(6171):367-370, May 1988.

[184] C. D. Meliza and Y. Dan. Receptive-field modification in rat visual cortex induced by paired visual stimulation and single-cell spiking. Neuron, 49(2):183-189, Jan. 2006.

[185] V. Pawlak, D. S. Greenberg, H. Sprekeler, W. Gerstner, and J. N. Kerr. Changing the responses of cortical neurons from sub- to suprathreshold using single spikes in vivo. eLife, 2, Jan. 2013.

[186] F. Wolf and T. Geisel. Universality in visual cortical pattern formation. Journal of PhysiologyParis, 97(2-3):253-264, Mar. 2003.

[187] D. L. Meinecke and A. Peters. GABA immunoreactive neurons in rat visual cortex. The Journal of Comparative Neurology, 261(3):388-404, July 1987.

[188] H. Markram, M. Toledo-Rodriguez, Y. Wang, A. Gupta, G. Silberberg, and C. Wu. Interneurons of the neocortical inhibitory system. Nature Reviews Neuroscience, 5(10):793-807, Oct. 2004.

[189] T. Binzegger, R. J. Douglas, and K. A. C. Martin. A quantitative map of the circuit of cat primary visual cortex. The Journal of Neuroscience, 24(39):8441-8453, Sept. 2004.

[190] D. Katzel, B. V. Zemelman, C. Buetfering, M. Wolfel, and G. Miesenböck. The columnar and laminar organization of inhibitory connections to neocortical excitatory cells. Nature Neuroscience, 14(1):100-107, Jan. 2011.

[191] A. Gupta, Y. Wang, and H. Markram. Organizing principles for a diversity of GABAergic interneurons and synapses in the neocortex. Science, 287(5451):273-278, Jan. 2000.

[192] X. Xu, K. D. Roby, and E. M. Callaway. Immunochemical characterization of inhibitory mouse cortical neurons: Three chemically distinct classes of inhibitory cells. The Journal of Comparative Neurology, 518(3):389-404, Feb. 2010.

[193] G. A. Ascoli, L. Alonso-Nanclares, S. A. Anderson, G. Barrionuevo, R. Benavides-Piccione, A. Burkhalter, G. Buzsáki, B. Cauli, J. DeFelipe, A. Fairén, D. Feldmeyer, G. Fishell, Y. Fregnac, T. F. Freund, D. Gardner, E. P. Gardner, J. H. Goldberg, M. Helmstaedter, S. Hestrin, F. Karube, Z. F. Kisvárday, B. Lambolez, D. A. Lewis, O. Marin, H. Markram, A. Muñoz, A. Packer, C. C. H. Petersen, K. S. Rockland, J. Rossier, B. Rudy, P. Somogyi, J. F. Staiger, G. Tamas, A. M. Thomson, M. Toledo-Rodriguez, Y. Wang, D. C. West, R. Yuste, G. A. Ascoli, L. Alonso-Nanclares, S. A. Anderson, G. Barrionuevo, R. Benavides-Piccione, A. Burkhalter, G. Buzsáki, B. Cauli, J. DeFelipe, A. Fairén, D. Feldmeyer, G. Fishell, Y. Fregnac, T. F. Freund, D. Gardner, E. P. Gardner, J. H. Goldberg, M. Helmstaedter, S. Hestrin, F. Karube, Z. F. Kisvárday, B. Lambolez, D. A. Lewis, O. Marin, H. Markram, A. Muñoz, A. Packer, C. C. H. Petersen, K. S. Rockland, J. Rossier, B. Rudy, P. Somogyi, J. F. Staiger, G. Tamas, A. M. Thomson, M. Toledo-Rodriguez, Y. Wang, D. C. West, and R. Yuste. Petilla terminology: nomenclature of features of GABAergic interneurons of the cerebral cortex. Nature Reviews Neuroscience, 9(7):557-568, July 2008. 
[194] B. A. McGuire, C. D. Gilbert, P. K. Rivlin, and T. N. Wiesel. Targets of horizontal connections in macaque primary visual cortex. The Journal of Comparative Neurology, 305(3):370-392, Mar. 1991.

[195] Z. F. Kisvárday, K. a. C. Martin, T. F. Freund, Z. Maglóczky, D. Whitteridge, and P. Somogyi. Synaptic targets of HRP-filled layer III pyramidal cells in the cat striate cortex. Experimental Brain Research, 64(3):541-552, Nov. 1986.

[196] C. Holmgren, T. Harkany, B. Svennenfors, and Y. Zilberter. Pyramidal cell communication within local networks in layer 2/3 of rat neocortex. The Journal of Physiology, 551(1):139 -153, Aug. 2003.

[197] A. M. Packer and R. Yuste. Dense, unspecific connectivity of neocortical parvalbumin-positive interneurons: A canonical microcircuit for inhibition? The Journal of Neuroscience, 31(37):1326013271, Sept. 2011.

[198] M. Galarreta and S. Hestrin. Electrical and chemical synapses among parvalbumin fast-spiking GABAergic interneurons in adult mouse neocortex. Proceedings of the National Academy of Sciences of the United States of America, 99(19):12438-12443, Sept. 2002.

[199] G. Silberberg and H. Markram. Disynaptic inhibition between neocortical pyramidal cells mediated by martinotti cells. Neuron, 53(5):735-746, Mar. 2007.

[200] M. Avermann, C. Tomm, C. Mateo, W. Gerstner, and C. C. H. Petersen. Microcircuits of excitatory and inhibitory neurons in layer $2 / 3$ of mouse barrel cortex. Journal of Neurophysiology, 107(11):3116-3134, June 2012.

[201] C. Kapfer, L. L. Glickfeld, B. V. Atallah, and M. Scanziani. Supralinear increase of recurrent inhibition during sparse activity in the somatosensory cortex. Nature Neuroscience, 10(6):743753, June 2007.

[202] R. Azouz, C. M. Gray, L. G. Nowak, and D. A. McCormick. Physiological properties of inhibitory interneurons in cat striate cortex. Cerebral Cortex, 7(6):534-545, Sept. 1997.

[203] J. A. Cardin, L. A. Palmer, and D. Contreras. Stimulus feature selectivity in excitatory and inhibitory neurons in primary visual cortex. The Journal of neuroscience : the official journal of the Society for Neuroscience, 27(39):10333-10344, Sept. 2007.

[204] A. M. Kerlin, M. L. Andermann, V. K. Berezovskii, and R. C. Reid. Broadly tuned response properties of diverse inhibitory neuron subtypes in mouse visual cortex. Neuron, 67(5):858871, Sept. 2010.

[205] K. Sohya, K. Kameyama, Y. Yanagawa, K. Obata, and T. Tsumoto. GABAergic neurons are less selective to stimulus orientation than excitatory neurons in layer II/III of visual cortex, as revealed by in vivo functional ca2+ imaging in transgenic mice. The Journal of Neuroscience, 27(8):2145-2149, Feb. 2007.

[206] C. A. Runyan, J. Schummers, A. Van Wart, S. J. Kuhlman, N. R. Wilson, Z. J. Huang, and M. Sur. Response features of parvalbumin-expressing interneurons suggest precise roles for subtypes of inhibition in visual cortex. Neuron, 67(5):847-857, Sept. 2010. 
[207] J. Zhuang, C. R. Stoelzel, Y. Bereshpolova, J. M. Huff, X. Hei, J.-M. Alonso, and H. A. Swadlow. Layer 4 in primary visual cortex of the awake rabbit: Contrasting properties of simple cells and putative feedforward inhibitory interneurons. The Journal of Neuroscience, 33(28):11372-11389, July 2013.

[208] W.-p. Ma, B.-h. Liu, Y.-t. Li, Z. J. Huang, L. I. Zhang, and H. W. Tao. Visual representations by cortical somatostatin inhibitory neurons - selective but with weak and delayed responses. The Journal of Neuroscience, 30(43):14371-14379, Oct. 2010.

[209] V. Bonin, M. H. Histed, S. Yurgenson, and R. C. Reid. Local diversity and fine-scale organization of receptive fields in mouse visual cortex. The Journal of Neuroscience, 31(50):18506 -18521, Dec. 2011.

[210] S. Schuett, T. Bonhoeffer, and M. Hübener. Pairing-induced changes of orientation maps in cat visual cortex. Neuron, 32(2):325-337, Oct. 2001.

[211] D. E. Whitney, S. Chandrasekaran, J. D. Flórez-Weidinger, S.-G. Kim, F. Wolf, and J. Crowley. Pairing-induced plasticity in ferret visual cortex depends on the local geometric map structure in the orientation preference map. In Neuroscience 2012, New Orleans, Louisiana, USA, Oct. 2012.

[212] L. Gödecke, D.-S. Kim, T. Bonhoeffer, and W. Singer. Development of orientation preference maps in area 18 of kitten visual cortex. European Journal of Neuroscience, 9(8):1754-1762, 1997.

[213] M. Kaschube, M. Schnabel, F. Wolf, and S. Lowel. Interareal coordination of columnar architectures during visual cortical development. Proceedings of the National Academy of Sciences, 106(40):17205-17210, Sept. 2009.

[214] A. Holtmaat, T. Bonhoeffer, D. K. Chow, J. Chuckowree, V. De Paola, S. B. Hofer, M. Hubener, T. Keck, G. Knott, W.-C. A. Lee, R. Mostany, T. D. Mrsic-Flogel, E. Nedivi, C. Portera-Cailliau, K. Svoboda, J. T. Trachtenberg, and L. Wilbrecht. Long-term, high-resolution imaging in the mouse neocortex through a chronic cranial window. Nature Protocols, 4(8):1128-1144, July 2009.

[215] Y. Loewenstein, A. Kuras, and S. Rumpel. Multiplicative dynamics underlie the emergence of the log-normal distribution of spine sizes in the neocortex in vivo. The Journal of neuroscience: the official journal of the Society for Neuroscience, 31(26):9481-9488, June 2011.

[216] A. J. Holtmaat, J. T. Trachtenberg, L. Wilbrecht, G. M. Shepherd, X. Zhang, G. W. Knott, and K. Svoboda. Transient and persistent dendritic spines in the neocortex in vivo. Neuron, 45(2):279-291, Jan. 2005.

[217] J. T. Trachtenberg, B. E. Chen, G. W. Knott, G. Feng, J. R. Sanes, E. Welker, and K. Svoboda. Long-term in vivo imaging of experience-dependent synaptic plasticity in adult cortex. Nature, 420(6917):788-794, Dec. 2002.

[218] M. Mank, A. F. Santos, S. Direnberger, T. D. Mrsic-Flogel, S. B. Hofer, V. Stein, T. Hendel, D. F. Reiff, C. Levelt, A. Borst, T. Bonhoeffer, M. Hübener, and O. Griesbeck. A genetically encoded calcium indicator for chronic in vivo two-photon imaging. Nature Methods, 5(9):805-811, Sept. 2008. 
[219] T.-W. Chen, T. J. Wardill, Y. Sun, S. R. Pulver, S. L. Renninger, A. Baohan, E. R. Schreiter, R. A. Kerr, M. B. Orger, V. Jayaraman, L. L. Looger, K. Svoboda, and D. S. Kim. Ultrasensitive fluorescent proteins for imaging neuronal activity. Nature, 499(7458):295-300, July 2013.

[220] M. G. Shuler and M. F. Bear. Reward timing in the primary visual cortex. Science, 311(5767):1606-1609, Mar. 2006.

[221] M. Frenkel, N. Sawtell, A. Diogo, B. Yoon, R. Neve, and M. Bear. Instructive effect of visual experience in mouse visual cortex. Neuron, 51(3):339-349, Aug. 2006.

[222] G. M. Ghose, T. Yang, and J. H. R. Maunsell. Physiological correlates of perceptual learning in monkey v1 and v2. Journal of Neurophysiology, 87(4):1867-1888, Apr. 2002.

[223] A. Schoups, R. Vogels, N. Qian, and G. Orban. Practising orientation identification improves orientation coding in v1 neurons. Nature, 412(6846):549-553, Aug. 2001.

[224] C. Picanço-Diniz, L. Silveira, M. de Carvalho, and E. Oswaldo-Cruz. Contralateral visual field representation in area 17 of the cerebral cortex of the agouti: A comparison between the cortical magnification factor and retinal ganglion cell distribution. Neuroscience, 44(2):325-333, 1991. 


\section{Acknowledgements}

I am extremely grateful to my supervisor and brilliant mentor Fred Wolf for allowing me to join his group and explore the exciting field of theoretical neuroscience. All his wisdom and experience in approaching scientific questions have been a role model for me and are reflected in every page of this thesis. Thanks.

The discussions that arose in the committee meetings with Detlev Schild and Stefan Treue have been an indispensable ingredient for this work and an immense encouragement for me to continue researching deeper into the field. Thanks.

A working environment like the one I had during these years at the Max Planck Institute for Dynamics and Self-Organization is unique. This was only possible due to the hard work of Theo Geisel, who spares no effort in making his department an amiable place to work. I am also very grateful - for their help in many different aspects of the life in front of a computer - to Yorck-Fabian Beensen and Denny Fliegner, and - for their patience in my endless travel reimbursement issues - to Ayse Bolik, Viktoria Nowak, Regina Wunderlich and Yvonne Reimann. The least I could do as gratitude was to water the plants of the department. Thanks.

During the years of the project I came across many superb scientist with whom I had the pleasure to interact. Dmitri Tsigankov was my first step-supervisor when Fred Wolf was not around and I learned a lot from him. Later during the thesis Wolfgang Keil joined the project and opened a world of interesting and productive discussions, helping me to clarify and mature many ideas. Thanks.

"Stone walls do not an office make". I had the privilege to share a room with wonderful people: Fabio Schittler-Neves and Dmitry Bibichkov in Bunsentrasse, and Wei Wei, Mirko Lukovic and Mantas Gabrielaitis in Fassberg. Manuel Schottdorf went through both the trouble of proof-reading part of the thesis and helping me to improve my skiing skills. Lars Reichl helped me to understand many details of the long-range interaction model. Lidia Rupp was always the first person to give me a smiley welcome to the institute every day. I am very grateful to all students and staff in the institute for their friendship. Thanks.

It is my belief that every theoretician working in neuroscience should have contact with the experimental side of this broad field. I had the fortune to enrich my scientific knowledge by collaborating in projects with two groups. Justin Crowley and David Whitney made my stay at the Carnegie Mellon Institute in Pittsburgh a great experience and I am happy that both the friendship and the collaboration are continued today. Jason Kerr and Vishnudev Ramachandra, from the Max Planck Institute of Biological Cybernetics in Tübingen, made it possible for me enter the field of 2-photon microscopy and provided an experimental counterpart to my theoretical work in rodents. Sadly most of the results of these projects are not included in the thesis due to time limitations, but I am sure our names 
will come together in a joint publication in the near future. Thanks.

The project to create a flexible database for all experimental measurements shared with the group gave me the unique chance to give a thoughtful look to the basics of organization and management of large data quantities. Although the development of the ADAMANT environment in collaboration with Timo Nachstedt, Marcus Schwamberger and Denny Fliegener was interrupted, it lays the foundation of future approaches in this direction. I am also indebted with Melanie Haas for sorting a large fraction of the data for this purpose. Thanks.

The work of a coordinator is best when you don't even notice something was coordinated. This is the case of Michael Hörner and Sandra Drube from the IMPRS Neurosciences. Due to their hard work the transition from Colombia to Germany was easy and enjoyable. Their support during this years was invaluable. Thanks.

Although it would be nice, a student is not only fed by his love for science. I am very grateful to Stefan Treue, Karin Peinemann and the NEUROSENSES program for the scholarship and the big support I received during my thesis. Thanks.

During this years in Göttingen I made many friends, and life here without them would be unimaginable. I learned a lot about the world from the students of the Neuroscience and Molecular Biology programs. Sharing experiences with them, paradoxically, made the world smaller and bigger simultaneously. Thanks.

The large latino community in Göttingen made this country feel like home, cooking delicious dishes and making a lot of noise. Their presence made the long winters less cold. It is difficult to find people that are up for everything with so much energy as they were. I hope the contact established here is maintained when the time comes to go back to Colombia. Thanks.

Words fall short to describe the crazy traveling team: Koray Kirli, Sinem Saka, Carlos Eduardo da Cunha, Helena Magliarelli, Andrés Flórez, Iris Finci and Melanie Haas. Discovering the world with them was extremely fun. There are still many places to explore. Thanks.

Europe has many things that are unique in the world. One of the most important for me are the metal festivals. Rain or shine, Wendy Liu and Melanie Haas joined me in many adventures that I hope will be repeated soon. Thanks.

The endless hours sitting in front of the computer were successfully balanced by a good dose of Capoeira. I am especially grateful to Fumaça, Curinga, Dum-dum, Careca, Batata and Fogueira for their patience and for teaching me everything I know. Thanks.

It is important to acknowledge my roots, without which I wouldn't be the person I am. Every visit to my friends back in Colombia made me feel as if time never passed. Hopefully it never will. I am greatly indebted to my family. Although not physically present during these years, their love and support were fundamental for every word of this thesis. There is not a single day I don't thank life for the parents and brother I have, Lope, Anne and Andrés. Whenever they are present, I feel like home. This thesis is their success, too. Thanks.

Melanie Haas, this thesis is dedicated to you. You are the Alpha and the Omega of people I am grateful to. You made everything possible and joined me in this dream. Your unconditional love and support during all these years are the reason I carry on. I love you. Thank you very much! 
